UNIVERSITE DU QUEBEC

\author{
MEMO IRE \\ PRESENTE A \\ L'UNIVERSITE DU QUEBEC A CHICOUTIMI \\ COMME EXIGENCE PARTIELLE \\ DE LA MAITRISE EN GESTION DES PMO
}

PAR

CAROLINE HUOT

B.A.A.

LA PLANIFICATION STRATEgIQUE DANS LES PME

EN PHASE DE PREDEMARRAGE: CAS DES ENTREPRISES DE SERVICES

AVRIL 1993 


\section{Bibliothèque}

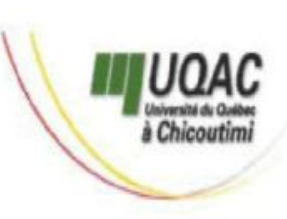

\section{Mise en garde/Advice}

Afin de rendre accessible au plus Motivated by a desire to make the grand nombre le résultat des results of its graduate students' travaux de recherche menés par ses research accessible to all, and in étudiants gradués et dans l'esprit des accordance with the rules règles qui régissent le dépôt et la governing the acceptation and diffusion des mémoires et thèses diffusion of dissertations and produits dans cette Institution, theses in this Institution, the I'Université du Québec à Université du Québec à Chicoutimi (UQAC) est fière de Chicoutimi (UQAC) is proud to rendre accessible une version make a complete version of this complète et gratuite de cette œuvre. work available at no cost to the reader.

L'auteur conserve néanmoins la The author retains ownership of the propriété du droit d'auteur qui copyright of this dissertation or protège ce mémoire ou cette thèse. thesis. Neither the dissertation or Ni le mémoire ou la thèse ni des thesis, nor substantial extracts from extraits substantiels de ceux-ci ne it, may be printed or otherwise peuvent être imprimés ou autrement reproduced without the author's reproduits sans son autorisation. permission. 


\section{SOMMA IRE}

De nos jours, la creation d'entreprises represente un stimulant economique non négligeable. De ce fait, il importe d'ameliorer nos connaissances relatives à ce phenomene afin de favoriser la naissance de nouvelles entreprises et egalement, leur survie, dans les meilleures conditions possibles. Dans cette perspective, certaines etudes ont eté réalisées et ont démontré l'existence d'une relation entre la phase de predemarrage d'une entreprise et les premieres annés de vie de cette entité economique. Hors, cette phase de predemarrage consiste, en quelque sorte, en la réalisation du premier processus de planification stratégique. Bien que la littérature soit relativement abondante concernant les plans d'affaires, peu d'etudes se sont intéressés à ce premier stade de vie de l'entreprise et plus specifiquement aux comportements adoptés par les entrepreneurs lors de cette premiere démarche de planification.

L'objectif de cette recherche vise donc a identifier et à evaluer les comportements de planification stratégique des entrepreneurs en phase de prédémarrage. A cette fin, un échantilion de 32 entrepreneurs ayant crée une entreprise de services au Saguenay-Lac-St-Jean, depuis moins d'un an, ont complété un questionnaire lors d'entrevues de groupes.

Globalement, les résultats de cette etude démontrent qu'en phase de prédemarrage, les entrepreneurs definissent la mission de l'entreprise et elaborent des objectifs à court terme. De plus, lors de l'analyse de 
l'environnement, ces créateurs d'entreprises privilégient certaines dimensions de l'environnement. Ainsi, à cette etape, les environnements sectoriel et interne les intéresssent davantage que l'environnement général. De façon plus détaillée, lors de l'analyse de l'environnement interne, ils concentrent leurs efforts de planification principalement sur l'aspect marketing et accordent un interet particulier à la variable qualité du service à offrir. Concernant l'environnement sectoriel, chacune des dimensions étudiees sont analysees à des degres comparables. Cependant, certaines différences apparaissent au niveau de l'analyse des variables liees à chacune de ces dimensions. Par ailleurs, la plupart des variables liées à l'environnement général sont analysées à des degrés equivalents par les entrepreneurs en phase de predemarrage.

De plus, on constate que les entrepreneurs consultent principalement leurs amis, les revues et journaux ainsi que les clients en vue d'obtenir des informations concernant la future entreprise et qu'ils s'intéressent de façon particulière, à la clientele, à l'économie et aux concurrents. Quant aux choix stratégiques privilégies par ces nouveaux entrepreneurs, ils consistent a offrir des services innovateurs et specialises, qui soient de qualité superieure. Ces services, offerts à des prix comparables à ceux des concurrents, s'adressent genéralement à une clientèle ciblee et visent un marché où la concurrence est faible.

Bref, on observe certaines differences entre les comportements de planification stratégique adoptes par les entrepreneurs en phase de 
prédémarrage et ceux proposes par la littérature sur les plans d'affaires. 


\section{REMERCIEMENTS}

La réallsation de cette recherche a necessitéla collaboration e't l'appui de nombreuse personnes. D'abord, j'aimerais remercier l'ensemble des collaborateurs externes de cette étude, ainsi que 1 es 32 entrepreneurs qui $y$ ont participé. De façon plus particulière, je remercie sincèrement mon directeur de memoire, M. Louis Dussault, pour les précieux conseils qu'il m'a prodigués, pour sa trés grande disponibilité et pour son soutien constant.

Je tiens également à souligner la collaboration de messieurs André Belley, Yves Lachance, Jean Lorrain et Jocelyn Perreault, tous professeurs en administration à l'université du Quebec, dont les conseils et la collaboration furent grandement appréciés. En outre, je remercie Mme Louise Sheehy pour l'aide et le temps qu'elle nous a accordé, lors du traitement des données statistiques.

En terminant, je remercie de façon particulière mon conjoint Carl, ma famille et mes proches pour la compréhension, l'aide et les encouragements dont j'ai pu bénéficier. Sans leur collaboration, la réalisation de cette recherche aurait été difficile. 
ChAPITRE 1: CADRE CONCEPTUEL . . . . . . . . . . . . . . . . . . . 5

1.1 PME ET ENTREPRENEUR . . . . . . . . . . . . . . . 6

1.2 PROCESSUS DE PLANIFICATION STRATEGIQUE . . . . . . . . 11

1.2.1 Modele de planification strategique . . . . 11

1.2.2 Etapes du processus de planification stratégique ............... 15

1.3 RECHERCHE SUR LA PLANIFICATION STRATEGIQUE DANS LES PME 20 1.3.1 Caractéristiques de la planification stratégique dans les pme ........ 20

1.3.2 Impact de la planification dans les PME . . 27

1.3.3 Contenu de la planification stratégique dans les PME ................ . . 30 1.3.3.1 Contenu des stratégies . . . . . 31 
1.3.3.2 Contenu de la planification stra-

tégique . . . . . . . . . . 34

1.3.4 Processus de planification stratégique dans les PME ..................... . . 36

1.4 PLANIFICATION STRATEgIQUE EN PHASE DE PRE-

DEMARRAGE . . . . . . . . . . . . . . . . . . . . . . 43

1.4.1 Phase de predemarrage . . . . . . . . . . 43

1.4.2 Plans d'affaires . . . . . . . . . 47

1.4.3 Recherches sur les entreprises en phase de

predémarrage . . . . . . . . . . . . . 51

CHAPITRE II: OBJECTIF ET HYPOTHESES DE RECHERCHE . . . . . . . . . . . . 56

CHAPITRE 111: METHODOLOGIE ........................ . 60

3.1 POPULATION .................. 61

3.1.1 Choix des entreprises de services . . . . . 62

3.2 TECHNIQUe D'ECHANTILlONNAGE . . . . . . . . . . 62

3.3 INSTRUMENT DE MESURE . . . . . . . . . . . . . . . . . 63

3.4 PROCEDURE D'EXPERIMENTATION . . . . . . . . . . . 66

3.5 METHODE D'ANALYSE . . . . . . . . . . . . . 67

CHAPITRE IV: PRESENTATION DES RESULTATS . . . . . . . . . . . . . . . . 68

4.1 CARACTERISTIQUES DE L'ECHANTILLON . . . . . . . . . 69

4.1.1 Répartition des entreprises par ville . . . 69

4.1.2 Répartition des entreprises par eecteur

d'activité ................ . . 70 
4.2 CARACTERISTIQUES DES ENTREPRISES . . . . . . . . . 72

4.2.1 Caractéristiques générales des entreprises . . 72

4.2.2 Caractéristiques liés aux activités de

l'entreprise . . . . . . . . . . . 72

4.3 CARACTERISTIQUeS DE L'EnTREPRENEUR . . . . . . . . . 75

4.3.1 Caractéristiques personnelles des entrepreneurs 75

4.3.2 Motivation et facteur declencheur . . . . . 78

4.3.3 Source de l'idée d'affaires . . . . . . 79

4.4 RESULTATS CONCERNANT LA PHASE DE PREDEMARRAGE... .81

4.4.1 Caractéristiques génerales de la phase de prédémarrage . . . . . . . . . . . 81

4.4.2 Connaissance du secteur d'activité et de

la gestion ................ 83

4.4.3 Comportements de gestion des entrepreneurs

liés à l'analyse des environnements général,

sectoriel et interne .. . . . . . . . 83

4.4.3.1 Comportements de gestion des

entrepreneurs liés à l'anal yse des

facteurs de l'environnement général. 85

4.4.3.2 Comportements de gestion des entrepre-

neurs liés à l'analyse des facteurs de

l'environnement sectoriel . . . 85

4.4.3.3 Opportunité et menaces de

l'environnement externe . . . . . 90 


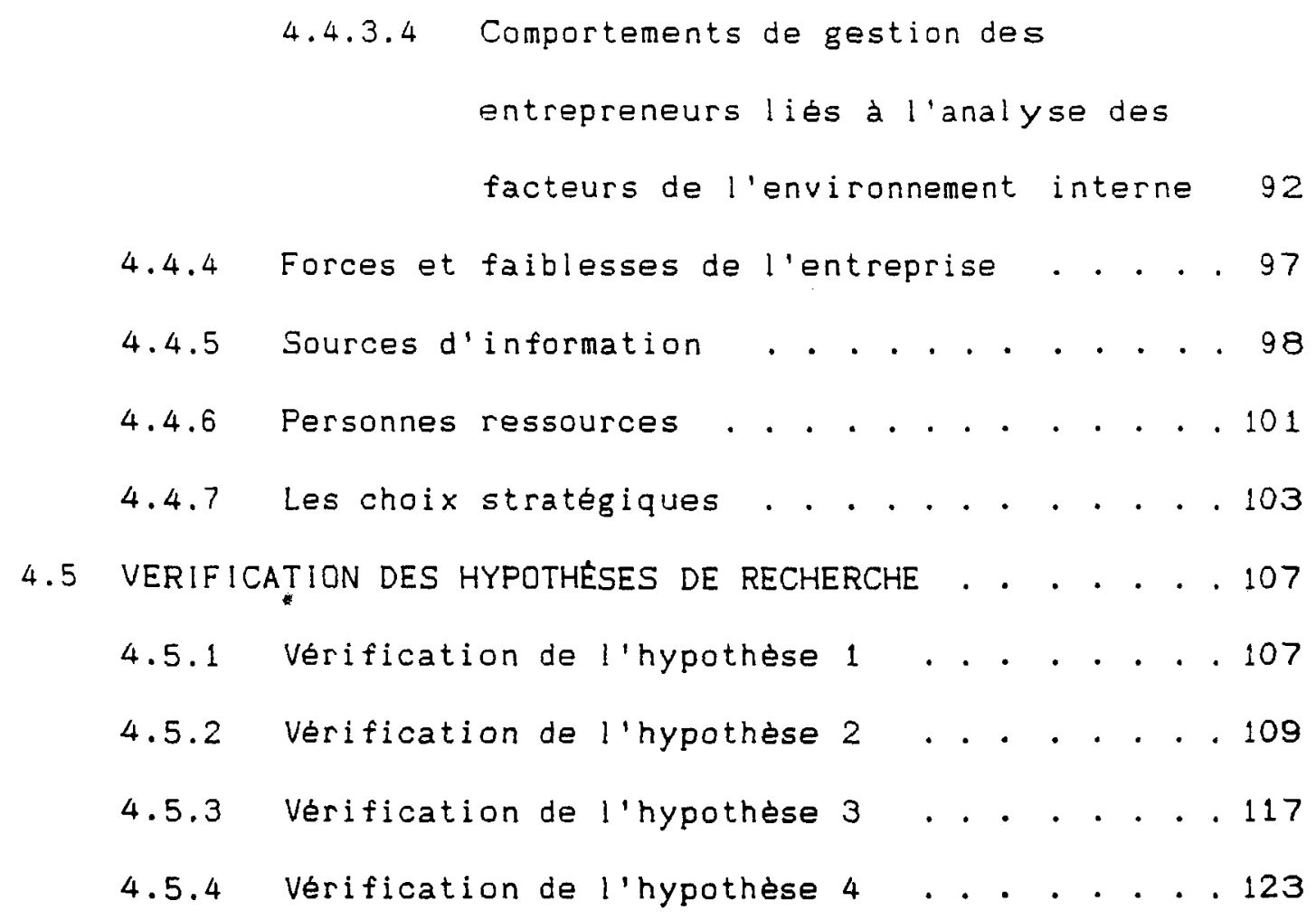

CHAPITRE V: DISCUSSION ET INTERPRETATION DES RESULTATS $\quad . \quad \cdot \quad . \quad$ • . . 126

CONCLUSION . . . . . . . . . . . . . . . . . . . . . . . . 136

BIBLIOGRAPHIE . . . . . . . . . . . . . . . . . . . . . . . . . 140

ANNEXE 1: QUESTIONNA IRE . . . . . . . . . . . . . . . . . . . . . 149

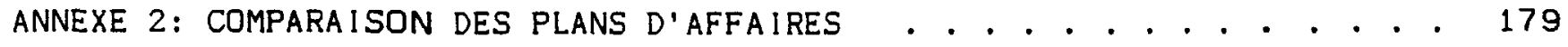

ANNEXE 3: SYNTHESE DES RECHERCHES CONSULTEES . . . . . . . . . . . . . 200 


\section{LISTE DES TABLEAUX}

Tableau 1 Modeles de planification strategique . . . . . . . . . . 12

Tableau 2 Modeles de planification stratégique adaptés à la PME . . 38

Tableau 3 Synthese du contenu d'un plan d'affaires . . . . . . . . 48

Tableau 4 Contenu du questionnaire . . . . . . . . . . . . . 65

Tableau 5 Répartition des entreprises de services échantillonnées parville........................ . . 70

Tableau 6 Répartition des entreprises de services echantillonnés par secteur d'activités . . . . . . . . . . . 71

Tableau 7 Caractéristiques générales des entreprises de services du Saguenay-Lac-St-Jean . . . . . . . . . . . . 73

Tableau $8 \quad$ Caractéristiques liées aux activités de l'entreprise . . . 74 Tableau 9 Caractéristiques personnelles des entrepreneurs . . . . . 76 Tableau 10 Motivation de l'entrepreneur à fonder son entreprise . . 80 Tableau 11 Source de l'idée d'affaires . . . . . . . . . . . . . 81 Tableau 12 Caractéristiques générales de la phase de prédémarrage . 82 Tableau 13 Connaissance du secteur d'activité et de la gestion de l'entrepreneur avant de décider de partir en affaires . . 84 Tableau 14 Comportements de gestion de l'entrepreneur lies à l'analyse des facteurs de l'environnement genéral lors de la phase de prédémarrage . . . . . . . . . . 86 
Tableau 15 Comportement de gestion de l'entrepreneur l'ís à l'analyse des facteurs de l'environnement sectoriel lors de 1 a phase de prédémarrage . . . . . . . . . . . . . . 87

Tableau 16 Facteurs considérés comme une opportunité ou une menace par l'entrepreneur lors de la phase de prédemarrage . . . 91

Tableau 17 Comportements de gestion de l'entrepreneur liés à l'analyse de l'environnement interne lors de la phase de predemarrage 93 Tableau 18 Forces et Faiblesses des entreprises de services en phase de prédémarrage . . . . . . . . . . . . . . . . . . 98

Tableau 19 Fréquence d'utilisation et degré d'interet des principales sources d'informations consultées par les entrepreneurs lors de la phase de prédemarrage . . . . . . . 100

Tableau 20 Degré de participation des personnes ressources lors de l'elaboration du projet d'entreprise . . . . . . . 103

Tableau 21 Raisons invoquées par les entrepreneurs pour contacter les personnes ressources lors de la phase de prédémarrage . 104 Tableau 22 Les choix stratégiques des entrepreneurs lors de 1 a phase de prédémarrage . . . . . . . . . . . . . 105

Tableau 23 Comparaison du degré d'analyse des environnements général, sectoriel et interne . . . . . . . . . . . . 108

Tableau 24 Comparaison entre le degré d'analyse des différents aspects de l'environnement interne ............. . 110

Tableau 25 Comparaison entre le degré d'analyse des différents facteurs de l'aspect marketing . . . . . . . . . . . . 112

Tableau 26 Comparaison entre le degré d'analyse des différents 
facteurs de l'aspect operationnel . . . . . . . . . . 114

Tableau 27 Comparaison entre le degré d'analyse des différents

facteurs de l'aspect humain . . . . . . . . . . 115

Tableau 28 Comparaison entre le degré d'analyse des differents

facteurs de l'aspect financier . . . . . . . . . . 116

Tableau 29 Comparaison entre le degré d'analyse des différentes

dimensions de l'environnement sectoriel . . . . . . . 118

Tableau 30 Comparaison entre le degré d'analyse des différents

facteurs lies à la disponibilite des ressources . . . . . . 119

Tableau 31 Comparaison du degré d'analyse des différents facteurs

liés a la dimension clients . . . . . . . . . . . 120

Tableau 32 Comparaison entre le degré d'analyse des différents

facteurs lies à la dimension fournisseurs . . . . . . . . 121

Tableau 33 Comparaison entre le degré d'analyse des différents

facteurs liés à la dimension concurrents . . . . . . . . 122

Tableau 34 Comparaison entre le degre d'analyse des différents

facteurs liés à l'environnement général . . . . . . . . . . 124 


\section{LISTE DES FIGURES}

FIGURE 1 Caractéristiques de la petite entreprise et des

impacts possibles sur la planification strategique . . . . 8

FIGURE 2 Modèle de Jauch et Glueck (1990) . . . . . . . . . . . . . 16

FIGURE 3 Classification des comportements strategiques selon

Miles et Snow (1978) . . . . . . . . . . . . . 32 


\section{INTRODUCT ION}

Les PME jouent un rôle essentiel dans l'economie du Québec. En 1987, elles représentalent $99,3 \%$ de toutes les entreprises québécoises et elles fournissaient 909,500 emplois (MIC,1988). Plusieurs facteurs peuvent expliquer ce phénomène. Parmi ces derniers, l'on retrouve les changements dans les politiques gouvernementales, le développement d'association de P.M.E., le développement de la recherche dans le domaine de l'entrepreneurship et le comportement des grandes entreprises (Julien et Marchesnay, 1987).

Dans ce contexte, de nouvelles entreprises ont vu le jour. Toutefois, l'on observe que les premieres années de vie d'une entreprise sont cruciales. En effet, des statistiques recentes indiquent de grandes variations dans le nombre de petites entreprises. Ainsi, 165980 entreprises furent crées en 1989 au Canada alors que 140054 disparaissaient résultant en une augmentation nette de 25926 entreprises par rapport a 1988. Cette augmentation représentant la plus petite progression depuis 1984 réflète l'accroissement des difficultés dues à l'environnement économique (Industrie, Sciences et Technologie Canada, 1991). Par ailleurs, une étude réalisée en 1985 par Dun \& Bradstreet nous démontre que 48,4 \% des faillites enregistrés au Canada étaient imputables à des entreprises de moins de 6 ans d'existence (Robidoux, 1980). 
En outre, les etudes de Mayer \& Golstein (1961) et de Wyant (1977), démontrent que le manque de planification constitue l'une des causes majeures de la faillite. Il semble que la meconnaissance de l'environnement joue également un rôle important à ce niveau. En effet, l'étude de Lalonde (1984) indique que le manque de connaissance de l'environnement entraine une méconnaissance des problèmes. De plus, Van Kirk et Noonan (1982) concluent que "l'incapacité à reconnaitre le changement" compte comme l'une des principales causes d'echec chez les petites entreprises.

Effectivement, les PME évoluent dans un environnement dynamique où la concurrence est tres forte et intense louverture des marches internationaux, etc.) et les marchés sont tres volatiles. Aussi, il devient important pour ces entreprises de gérer stratégiquement (Noël, 1989).

Mais que connaissons nous de la planification strategique dans les PME? Certaines etudes identifient quelques aspects specifiques de la planification stratégique dans les P.M.E. De manière générale, nous savons qu'en contexte de petites entreprises la planification strategique est non-structurée, irrégulière, non-formalisée et plutôt réactive que proactive (Robinson et Pearce, 1984). On a également constate que les petites entreprises planifient generalement sur une courte periode s'étendant de $\mathbf{s i x}(6)$ mois à deux (2) ans (Robinson et littlejohn, 1981).

Par ailleurs, l'application de l'approche contingentielle en matiere de planification stratégique devient une préccupation pour certains 
chercheurs. A cet effet, Robinson Logan et Salem (1986) mentionnent le fait qu'il est difficile de généraliser les pratiques managériales à toutes les situations organisationnelles. Parmi les facteurs de contingences étudiés, Vozikis \& Glueck (1980) ont démontré que le stade de développement d'une entreprise avait une influence sur le formalisme et sur les conditions de réalisations de la planification stratégique. Lindsay \& Rue (1980), quant à eux, considerent que le stade de développement est un important facteur de contingence en ce qui concerne le processus de planification à long terme.

Toutefois, bien que les ecrits soient nombreux sur le sujet, peu de recherches se sont intéressées de manière spécifique à la planification stratégique dans les entreprises en phase de prédémarrage. Pourtant, la phase de prédemarrage semble influencer le succès futur de l'entreprise (Gill, 1985),

C'est donc a partir d'observations telles: l'influence possible du stade de développement sur le processus de planification, l'ampleur du phénoméne de démarrage d'entreprises, et le taux de faillite élevé chez les jeunes entreprises, que nous formulons la question suivante:

Quels sont les comportements de planification stratégique adoptes par les futurs entrepreneurs lors de la phase de prédémarrage d'une entreprise ? 
Cette question nous apparait intéressante dans la mesure où l'on considère que la phase de demarrage d'une entreprise est en relation avec la capacité à reconnaitre et à resoudre les problemes intervenus au cours de la phase de prédemarrage. C'est dire que l'intensité d'un probleme augmente si celui-ci n'est pas résolu ou correctement estimé en phase de prédemarrage (Fourcade, 1986). Ainsi, le processus de planification suivi en phase de predémarrage pourrait influencer la vie future de l'entreprise puisqu'il vise, entre autre, a identifier et évaluer les problèmes ainsi qu'à envisager leurs solutions. La pertinence de notre question réside donc dans la perspective d'accroitre le taux de survie des jeunes entreprises.

Un autre aspect intéressant concernant l'influence de la phase de prédémarrage sur les périodes de vie subséquentes de l'entreprise ressort d'une étude réalisée par Shuman et Seeger (1986). En effet, les auteurs ont démontré que les dirigeants ayant preparé un pland'affaires sont plus enclins à utiliser les elements majeurs du processus de planification lorsque l'entreprise est en opération.

En résumé, en référence aux faits énumérés précédemment nous tenterons d'apporter quelques éléments de réponse a cette question et ce, afin d'accroitre nos connaissances sur la planification stratégique lors de la phase de prédémarrage d'une entreprise. 
CHAPITRE I

CADRE CONCEPTUEL 


\section{CHAP ITRE I}

\section{CADRE CONCEPTUEL}

Ce chapitre présente l'ensemble des connaissances pertinentes liées a la problématique étudiée dans le cadre de cette recherche soit, la planification stratégique dans les entreprises en phase de prédemarrage.

Ce cadre conceptuel se compose essentiellement de quatre (4) parties. La première de ces parties présente le processus de planification stratégique. La deuxieme partie identifie les spécificités de la petite entreprise ainsi que les caracteristiques de l'entrepreneur alors que la troisieme partie analyse les résultats de différentes recherches sur la planification stratégique et opérationnelle en contexte de PME. Finalement, la derniere partie traite de la phase de prédemarrage d'une entreprise et de la planification stratégique associée au développenent du plan d'affaires.

\subsection{PME ET ENTREPRENEUR}

Les PME représentent au Québec plus de $99 x$ des entreprises IMIC, 1988) et bien qu'on les ait longtemps considérées comme des modeles réduits d'entreprise, l'on constate aujourd'hui qu'elles possedent leur propre réalité et leur propre existence (Julien et Marchesnay, 1987). 
A ce propos, Julien et Marschesnay (1987) identifient quelques unes des caractéristiques qui font de la PME une entitédistincte. Ces caractéristiques et leurs impacts sur la planiflcation stratéglque sont d'allleurs présentés à la flgure 1 de la page suivante.

Ces caractéristiques influencent le processus de planification stratéglque de différentes maniéres. Dans un premier temps, la forte centralisation ou personnalisation de la gestion nous laisse entrevoir l'importance du rôle joué par le dirigeant au niveau des décisions stratéglques. Alnsl, les objectifs personnels de l'entrepreneur deviennent des eléments déterminants dans le choix des objectifs de l'entreprise (Thurston, 1984).

Quant à la faible spécialisation du travall, elle laisse aux individus plus d'initiative et favorise une plus grande implication de ces derniers dans la détermination des plans de charges. Cette caracteristique constitue un avantage considerable en période de changements accélérés pulsqu'elle permet plus de souplesse. Cette grande souplesse est également favorisée par un processus de décision intuition-décisionaction (Julien et Marchesnay, 1987). A ce propos, Shrader, Mulford et Blackburn(1989) rejoignent Julien et Marchesnay en affirmant que dans la petite entreprise, le processus de décision fait généralement appel à l'intuition, a la présence d'esprit et à l'expérience du dirigeant. Julien et Marchesnay (1987) précisent de plus, que la souplesse structurelle des petites entreprises favorise une meilleure adaptation aux évolutions du marché et leur permet de profiter de la segmentation des 
Figure 1

Caractéristiques de la petite entreprise et des impacts possibles sur la planification stragegique

\begin{tabular}{|c|c|}
\hline CARACTERIST I QUES & $\begin{array}{l}\text { IMPACTS SUR LA PLANIF ICATION } \\
\text { STRATEGIQUE }\end{array}$ \\
\hline $\begin{array}{l}\text { Centralisation ou personna- } \\
\text { lisation de la gestion }\end{array}$ & $\begin{array}{l}\text { L'entreprise peut etre identifiee } \\
\text { a la direction; } \\
\text { Processus de décision simple. }\end{array}$ \\
\hline Faible spécialisation du travail & $\begin{array}{l}\text { Plus d'initiative aux personnes; } \\
\text { Permet plus de souplesse. }\end{array}$ \\
\hline $\begin{array}{l}\text { Processus de décision selon le } \\
\text { schéma intuition-décision-action }\end{array}$ & Stratégie implicite et trés souple. \\
\hline $\begin{array}{l}\text { Systeme d'information interne } \\
\text { peu complexe }\end{array}$ & Permet une diffusion rapide. \\
\hline $\begin{array}{l}\text { Systeme d'information externe } \\
\text { simple }\end{array}$ & $\begin{array}{l}\text { Marché proche, pas besoin d'étude } \\
\text { de marché complexe; } \\
\text { Perception du changement peut etre } \\
\text { rapidement saisie. }\end{array}$ \\
\hline $\begin{array}{l}\text { Recherche d'un environnement } \\
\text { stable }\end{array}$ & $\begin{array}{l}\text { Peu de poids sur l'environnement; } \\
\text { Recherche de crénaux. }\end{array}$ \\
\hline
\end{tabular}

Inspiré de Julien, P.A. et Marchesnay, M., La petite entreprise, 1987 
marches en croissance. Les auteurs ajoutent que les systemes d'information interne et externe relativement simples permettent une circulation plus rapide de l'information augmentant ainsi la capacite d'adaptation de ces petites organisations.

Par ailleurs, Van Kirk et Noonan (1982) se sont egalement intéressés aux particularités des PME. Ils concluent que la flexibilite, les frais généraux souvent moins élevés et l'impact plus direct d'une bonne gestion sur les profits constituent les principaux avantages reliés à la PME alors que la difficulté à mantenir un équilibre financier, les lignes de produits et les ressources limités ainsi que la difficulté a obtenir des informations concernant le marché représentent les principaux inconvenients.

Finalement, l'analyse des spécificités de la PME met en évidence le peu d'influence qu'exerce ce type d'entreprise sur son environnement ainsi que le rôle prédominant de l'entrepreneur qui, par son omniprésence, influence grandement le processus de planification stratégique.

La grande influence de l'entrepreneur est une réalité qui s'applique également en contexte de création d'entrerpise. D'ailleurs Shapero (1975) dans son modele, affirme que la creation d'entreprise repose d'abord sur la presence d'individus qui ont une certaine prédisposition à agir et que cette predisposition est fonction de certaines caracteristiques psychologiques (Belley, 1987). Donc, pour qu'une entreprise soit 
crée, il faut qu'un ou plusieurs individus possedant certaines caracteristiques agissent en conséquences.

Certaines études (Garnier et Gasse, 1986; Belley, 1987) se sont intéressées aux caractéristiques personnelles et psychologiques de l'entrepreneur et, de leurs conclusions, l'on retient les élements suivants. Parmi les caracteristiques personnelles etudiees, le niveau de scolarité et l'expérience sur le marché du travail ont recu une attention particulière. Ainsi, l'on considere que l'entrepreneur possède un niveau d'éducation formelle relativement bas, bien que des etudes recentes tendent à démontrer certaines améliorations à ce niveau. Par ailleurs, il semble que l'expérience sur le marché du travail, particulièrement au sein de PME, favorise l'entrepreneurship. Quant aux caracteristiques psychologiques, on dénote chez l'entrepreneur la présence de besoins d'accomplissement, d'indépendance et d'autonomie élevés, d'une grande créativité, d'un bon sens de l'initiative, d'une grande confiance en soi ainsi qu'une forte dose de motivation et d'energie.

En résumé, la PME se caractérise par l'omniprésence de l'entrepreneur, individu indépendant, qui genéralement centralise l'ensemble des décisions de gestion. Ceci est particulièrement vrai lors de la phase de prédémarrage où le ou les entrepreneurs sont pratiquement les seuls intervenants. C'est donc eux qui identifient l'idée d'affaires, recherchent l'information et qui réunissent et s'approprient les ressources en vue de creer l'entreprise. En un mot, ils representent les stratéges de 
l'entreprise et en tant que tel, ils sont responsables de l'elaboration des premiers plans stratégiques et operationnels de la future entreprise.

\subsection{PROCESSUS DE PLANIFICATION STRATEgIQUE}

La creation d'une entreprise est un processus relativement complexe par lequel l'entrepreneur doit identifier une idé d'affaires et en évaluer la faisabilite afin de déterminer si elle représente une opportunité intéressante (Belley, 1987). Cette démarche s'apparente fortement au processus de planification stratégique, processus sur lequel repose l'essentiel de notre problématique. Aussi, dans cette section, nous analyserons certains modeles de planification strategique puis, nous décrirons en détail chacune des composantes du modele retenu.

\subsubsection{Modele de planification stratégique}

A ce stade, il nous apparast important de préciser que le concept de planification stratégique a été développé chez la grandes entreprises et ce n'est que recemment que les chercheurs se sont intéressés a la planification stratégique en contexte de petites entreprises. Ainsi les quelques modeles normatifs consultés (Ansoff, 1974; Jauch et Glueck, 1990; Rue et Holland, 1986; Wheelen et Hunger, 1990; Martinet, 1983) sont élaborés dans une perspective de grandes organisations (tableau 1 ). 
Tableau 1

Modèle normatifs de planification stsatégique

\begin{tabular}{|c|c|c|c|c|}
\hline $\begin{array}{l}\text { Ansolf } \\
1974\end{array}$ & $\begin{array}{l}\text { Martinet } \\
1983\end{array}$ & $\begin{array}{l}\text { Rue } 1 \text { Holland } \\
\qquad 1986\end{array}$ & $\begin{array}{l}\text { Wheeten \& Hunger } \\
1990\end{array}$ & $\begin{array}{c}\text { Jauch \& Gluect } \\
1990\end{array}$ \\
\hline $\begin{array}{l}\text { Definition al twis des } \\
\text { otiectilis }\end{array}$ & $\begin{array}{l}\text { Mission el frithé } \\
{ }^{3} \text { Obiectits genérats }\end{array}$ & 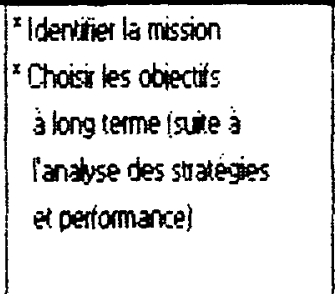 & $\begin{array}{l}\text { Mission } \\
\text { Xobiectifs }\end{array}$ & 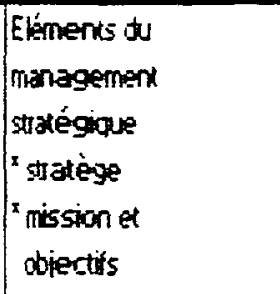 \\
\hline 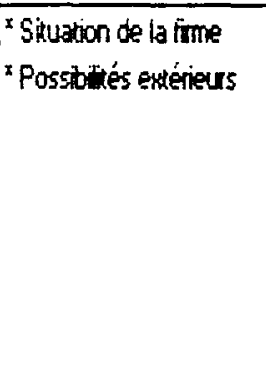 & 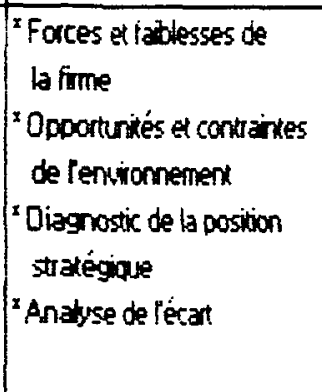 & 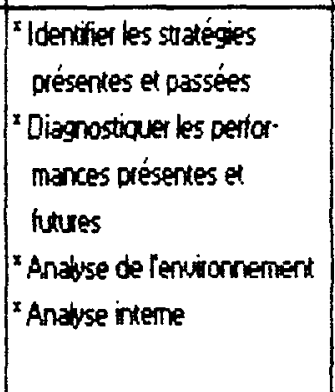 & \begin{tabular}{|l|} 
Esterns \\
"Enviornement social \\
" "Task envionnemen" \\
(Enviomement immediat) \\
Interne \\
"Stucture \\
"Culure \\
"Resscuces
\end{tabular} & 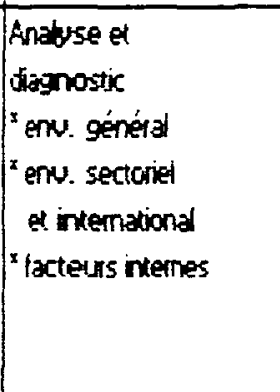 \\
\hline 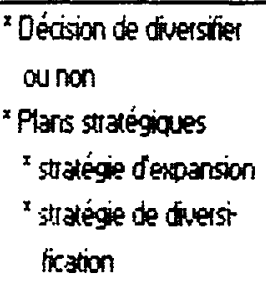 & $\begin{array}{l}\text { "Rectherche des statégies } \\
\text { S Sétection de la statége } \\
\text { eficace }\end{array}$ & 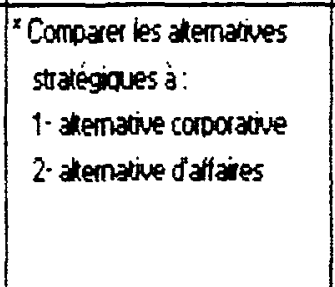 & $\begin{array}{l}\text { Surtégias } \\
\times \text { Poltiques }\end{array}$ & 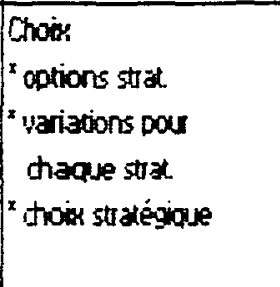 \\
\hline $\begin{array}{l}\text { "Redistibution des } \\
\text { lessouces } \\
\text { Rechectre dine } \\
\text { synergie }\end{array}$ & 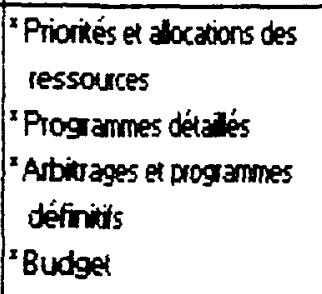 & $\begin{array}{l}\text { "Stragigies fonctionneles } \\
{ }^{x} \text { Facteuss organisationnets }\end{array}$ & $\begin{array}{l}{ }^{x} \text { Progammes } \\
{ }^{x} \text { Budget } \\
{ }^{x} \text { Procédues }\end{array}$ & $\begin{array}{l}\text { Implantation } \\
\text { ressources et stur. } \\
\text { ture } \\
\text { politiques, plans at } \\
\text { adninistation }\end{array}$ \\
\hline Rétraaction & $\begin{array}{l}\text { "Réatission et révision } \\
\text { stratégique }\end{array}$ & Evaluation et contrôte & $\begin{array}{l}\text { "Evaluation et contióle } \\
\text { " Performance }\end{array}$ & $\begin{array}{l}\text { Evaluation et contrồle } \\
\text { "Rétoaxtion } \\
\text { "Anticiostion }\end{array}$ \\
\hline
\end{tabular}


L'un des premiers modeles de décision stratégique appliqué au domaine de la gestion a été développé par Ansoff (1974). Ce modele s'inspire principalement de la doctrine et de la methodologie de prise de décision d'ordre militaire et d'etudes resultant d'initiatives d'entreprises. Pour Ansoff $(1974)$, les décisions stratégiques consistent essentiellement à choisir pour l'entreprise une combinaison de produits et de marchés a partir desquels on détermine les activités de l'entreprise. Ceci implique généralement une redistribution des ressources et la recherche d'un effet de synergie.

Dans son modele, Martinet (1983), tout comme Ansoff(1974), associe les décisions stratégiques au choix de couple produits-marches. Cependant, la particularite de ce modele en comparaison de celui de Ansoff (1974), est de considerer la mission ou finalitéde l'entreprise ainsi que l'ecart entre la position stratégique actuelle de l'entreprise et la position souhai tée.

Rue et Hol land (1986), quant à eux, présentent un modele s'apparentant à celui de Martinet (1983). Contrairement à ce dernier, l'analyse des stratégies passés et présentes précéde l'analyse des environnements internes et externes. De plus, Rue et Holland (1986) sont beaucoup moins explicites que Martinet (1983) concernant l'etape d'implantation des strategies.

Pour sa part, le modele de theelen et Hunger (1990), bien qu'interessant, ne présente pas de particularité si ce n'est qu'il apporte 
davantage de precision quant a l'analyse des environnements interne et externe.

Parallèlement, Jauch et Glueck (1990) ont développé un modèle de planification strategique qui lie les avantages stratégiques de l'entreprise aux défis de l'environnement (Cóté et al.,1991). Les auteurs presentent leur modele comme un processus de "management stratégique", concept qu'ils définissent de la façon suivante:

\begin{abstract}
Le management stratégique est un ensemble de decisions et d'actions qui conduisent au développement d'une stratégie efficace ou de stratégies qui aident a atteindre les objectifs de l'entreprise. Le processus de management stratégique est la façon selon laquelle les strateges déterminent les objectifs et prennent des décisions strategiques. (Jauch et Glueck, 1990, p.9)
\end{abstract}

Dans l'esprit de Jauch et Glueck (1990), les termes "planification stratégique" et "management stratégique" semblent avoir la meme signification. Par ailleurs, le processus de planification strategique conçu par Jauch et Glueck (1990) nous apparait come l'un des plus complets. En effet, seul ce modele intègre "les strateges" aux elements du management stratégique, ce qui, en contexte de PME, présente un intéret particulier compte tenu du râle déterminant qu'occupe l'entrepreneur dans la gestion de son entreprise (Julien et Marchesay, 1987). En outre, Jauch et Glueck (1990), considèrent que les decisions strategiques concernent principalement trois (3) élements soit: le produit lou service), le marché et les activités ou fonctions de l'entreprise. De 
plus, Jauch et Glueck (1990) accordent une importance particullere à l'implantation de la stratégie, laquelle précise la maniére dont les choix stratégiques deviendront opérationnels. Ces observations justifient le choix de Jauch et Glueck (1990) comme modele de référence dans le cadre de cette etude.

\subsubsection{Etapes du processus de planification stratégique}

Tel que mentionné précédemment, les modeles de processus de planification stratégique reposent sur des bases relativement similaires. Cependant, le modele de Jauch et Glueck (1990) résume bien l'ensemble de ces modèles. De plus, il a la particularité d'accorder une place privilégié au stratège dans le processus de planification stratégique. Ce modèle tel que représenté à la figure 2 se compose de cinq (5) étapes soit: les élements du management stratégique, l'analyse et le diagnostic, le choix, l'implantation et l'evaluation. Bien qu'elles soient présentées de manière séquentielle, ces étapes sont en réalité intégrées puisque chacune d'entre elles a une influence sur les autres.

Dans la première partie de leur modele, Jauch et Glueck (1990) identifient deux éléments de management stratégique soit: les stratéges ainsi que la mission et les objectifs de l'entreprise. Dans un premier temps, les auteurs insistent sur le rôle-clé du directeur général au niveau du processus de management stratégique. Selon eux, "les dirigeants au sommet de l'entreprise... sont les premiers responsables de la survie et 


\section{Flgure 2}

Modéle de Jauch et Glueck (1990)

Déterminer la mission, les buts et les valeurs de la firme et ses principaux preneurs de décisions

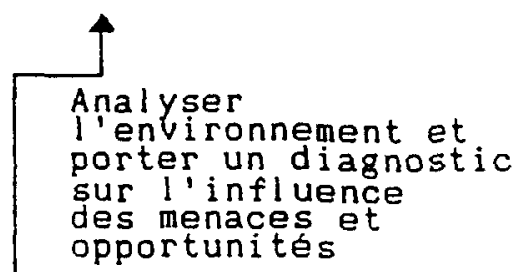

Examiner les forces et les faiblesses de la firme et porter un diagnostic sur elles

Considérer des options variees et s'assurer que la stratégie appropriée est choisie

lar a ce que les plans, les politiques. la structure et le style administratif solent bien assortis avec la stratégie

S'assurer que la stratégie et son implantation

permettront

d'atteindre

les objectifs
Les éléments du management stratégique

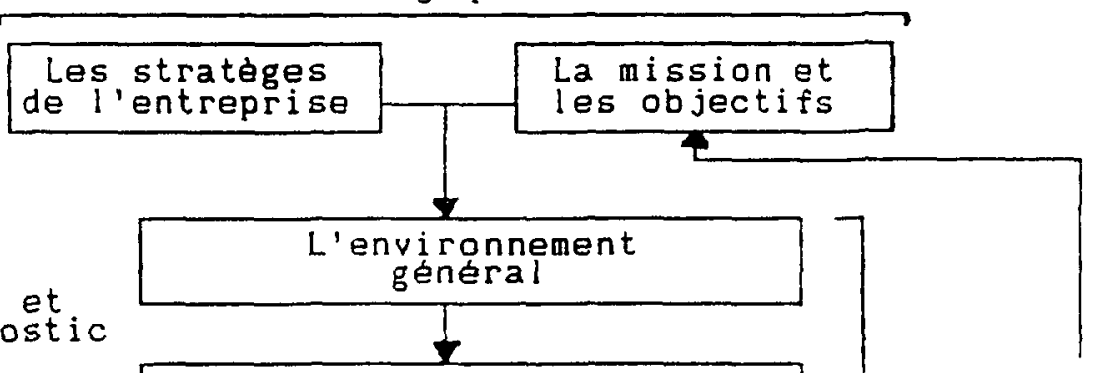

L'analyse et le diagnostic sectoriel et international

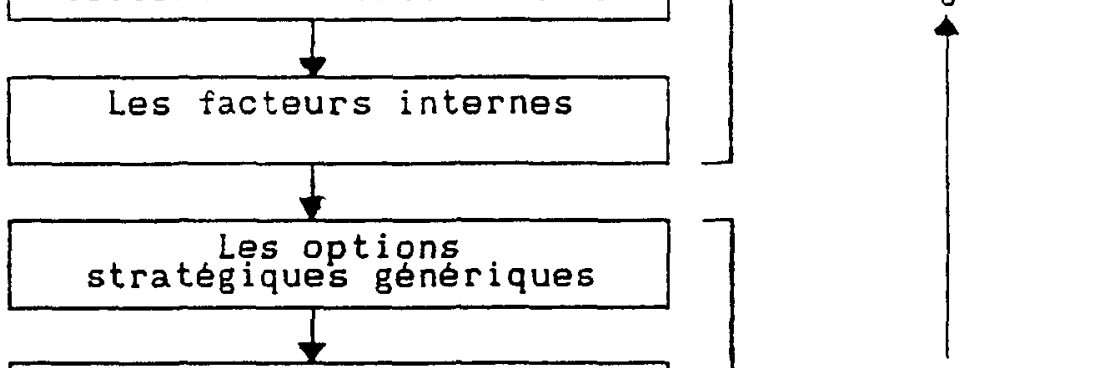
Le choix chaque strategie

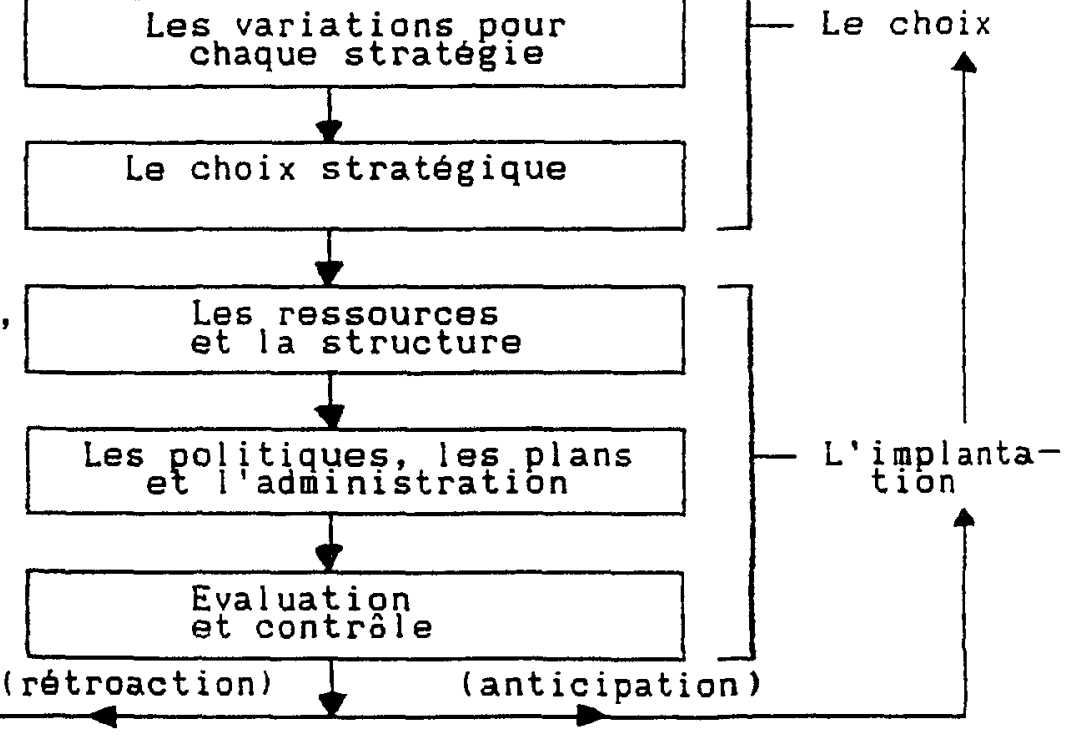

Source: Jauch, Lawrence R. et Glueck, Will iam F., Management stratégique et pol itique générale, 1990, p.10 
du succès d'une entreprisen. Ainsi, ces auteurs considerent que l'application pure et simple de theorie s'avere impossible et que les dirigeants doivent généralement faire appel à leur intuition et à leur jugement pour prendre les décisions d'ordre stratégique.

Dans un deuxieme temps, les auteurs accordent une importance particuliere a la mission et aux objectifs, qui definissent la nature de l'entreprise et servent de cadre pour la réalisation des étapes subséquentes du processus. La mission définit le secteur d'affaires de l'entreprise en référence à trois éléments soit: les produits lou servicesl, qui sont constitues de la valeur ajouté que l'on désire vendre au client; les marchés, qui réfèrent à des classes ou types de clients ou à des régions où le produit (ou service) est vendu; et les fonctions de l'entreprise, qui concernent les technologies de meme que les processus (et activités) utilisés pour ajouter de la valeur. Quant aux objectifs, Jauch et Glueck (1990) estiment que les strateges doivent non seulement definir des objectifs généraux et des objectifs specifiques mais ils doivent également établir un ordre de priorité dans leur réalisation.

Dans la deuxième partie de leur modile, Jauch et Glueck (1990) présentent l'etape d'analyse et de diagnostic au cours de laquelle sont analysés les environnements général, sectoriel, international et interne (ou facteurs internes) de l'entreprise.

L'environnement général est lié à l'ensemble des facteurs socioéconomiques, technologiques et gouvernementaux tandis que l'environnement sectoriel ou industriel prend en consideration des facteurs tels la clientele, les concurrents, les facteurs démographiques et géographiques ainsi que la disponibilité et le coût des matières premières et des emballages, de l'énergie, de l'argent et de la main d'oeuvre. L'envi- 
ronnement international quant à lui, considerre l'ensemble de ces facteurs mais dans une perspective internationale en vue d'analyser les menaces et opportunités présentes dans les marchés extérieurs.

La troisieme etape du processus vise essentiellement a effectuer des choix strategiques et ce, de façon rationnelle en considerant les menaces et opportunites de l'environnement ainsi que les forces et faiblesses de l'entreprise. A cette fin, le modele propose deux étapes soit: la formulation d'un nombre raisonnable d'options stratégiques avec leurs différentes variations et, le choix de la meilleure stratégie.

Dans cette optique, Jauch et Glueck (1990) identifient quatre (4) types de stratégies soit: la stabilite, l'expansion, le retraitet la stratégie combinée. Cette classification de stratégies etant difficilement applicable au contexte de la PME en phase de prédémarrage, nous avons dú référer à un autre auteur pour identifier des stratégies mieux adaptées au contexte de notre étude. Ainsi, Porter (1986), présente trois (3) stratégies de base permettant d'atteindre des résultats supérieurs à la moyenne soit: la domination par les coûts, la différenciation et la concentration. La donination par le coits consiste essentiellement à produire á coüts peu ́levés, à viser une cible large et à servir de nombreux segments. Tandis que par la différenciation, une entreprise mise sur la particularité de certaines dimensions fort apprécies des clients. La différenciation peut concerner le produit, le systeme de distribution, l'approche marketing et bien d'autres facteurs. La concentration, quant à elle, consiste à choisir un segment ou un groupe de segments cibles et à développer une stratégie sur mesure pour les servir a l'exclusion des autres. 
Ainsi, les stratéges doivent selectionner parmi les grandes options strategiques possibles, celle qui convient le mieux aux objectifs de l'entreprise. La décision implique que l'on se concentre surquelques options, que l'on considere les facteurs de sélection et que l'on évalue les options par rapport à ces criteres. Le choix de la stratégie doit prendre en considération les 1 imites de capacités relatives aux ressources, à la structure, aux politiques et au systeme administratif.

La quatrieme étape du processus de planification, l'implantation, permet de rendre opérationnelle la stratégie choisie par le développement d'une structure appropriee, de plans et de politiques. Jauch et Glueck (1990) propose donc qu'une evaluation des ressources requises soit effectuee en vue d'optimiser leur affectation. Par la suite, afin d'assurer une plus grande efficacite, les strateges devront adapter $1 a$ structure administrative aux changements proposés. Finalement, des plans et des politiques devront etre etablis pour guider les décisions des dirigeants fonctionnels.

La cinquieme et derniere étape du processus, l'evaluation et le contrôle, vise à s'assurer que la stratégie choisie est bien iaplantée et qu'elle permet d'atteindre les objectifs poursuivis.

Le processus de planification stratégique constitue done une demarche relativement complexe qui exige un minimum d'habileté et de temps. Aussi, une application intégrale de ces modeles thériques peut s'averer difficile en pratique. 


\subsection{RECHERCHE SUR LA PLANIFICATION STRATEGIQUE DANS LES PME}

La planification stratégique a fait l'objet de nombreux écrits et les modeles de base présentés précedemment en sont des exemples concrets. Cependant, ces théories, de façon générale, ont été développées dans une optique de grandes entreprises. Hors, la PME, comme nous l'avons déja mentionné, possède sa propre réalité et ses propres caractéristiques. Ces observations nous incitent a croire que le processus de planification strategique peut présenter certaines variations dans ces entreprises. L'objectif de cette section consiste donc à etudier les differentes dimensions de la planification stratégique dans les PME. Pour faciliter la comprehension et la comparaison des faits observes, nous avons regroupé le tout selon quatre themes inspirés de Robinson et Pearce (1984) soit: les caractéristiques de la planification stratégique, l'impact de la planification stratégique, le contenu de la planification strategique et, finalement, le processus de la planification stratégique.

\subsubsection{Caractéristiques de la planification stratégique dans les pme}

Dans leur article sur l'etat de la recherche sur la planification dans les petites entreprises, Robinson et Pearce (1984) constatent l'existence d'un consensus à l'effet que le processus complet de planification n'existe pas dans les PME. Les principales raisons émises pour expliquer cette absence sont le manque de temps, le manque de connaissances du processus de planification strategique, le manque d'expertise specialisee ainsi que le manque de confiance et d'ouverture des dirigeants. 
Parallelement, Sexton et Van Auken (1982) ont cherché a classifier les entreprises selon leur niveau de planification stratégique les résultats de leur recherche montrent que seulement $18 \%$ des répondants sont considérés comme des planificateurs. L'étude révele donc un niveau anémique de planification stratégique dans l'échantillon 1357 entreprises) puisque moins du quart des entreprises étudiées font des efforts pour anticiper les changements dans les ventes et les profits et pour articuler des plans operationnels. Nous croyons cependant qu'il faut etre prudent quant a l'interprétation de ces resultats etant donné le contenu restreint des mesures utilisés (seulement 4 questions) pour évaluer le niveau de planification stratégique.

Shrader, Mulford et Blackburn (1989), quant à eux, ont observé que $67 x$ des entreprises etudiées $(31$ manufactures, 35 detaillants et 31 entreprises de services) ne faisaient pas de planification strategique sur une periode excedant un $(1)$ an et que toutes les entreprises engagees dans un processus de planification stratégique (33x de l'échantillon) avaient développé des plans operationnels. Ils ont constaté également qu'aucune différence significative n'existait au niveau de la planification stratégique entre les differents secteurs etudies. Enfin, lors de cette recherche, les raisons mentionnées par les repondants pour expliquer l'absence de planification stratégique sont le manque de temps, le manque d'habilete, les coûts élevés et l'environnement imprévisible. Ceci rejoint les conclusions de Robinson et Pearce (1984).

A la lumiére de ces informations, nous constatons que peu d'entreprises utilisent le processus de planification strategique dans son intégrité, tel que développé dans les modeles normatifs, et ce, principalement en raison d'un manque de temps. Alors, quelles sont les caracteristiques de la planification telle que pratiquee dans les PME? 
Dans un article de Robingon, Logan et Salem(1981) traitant dea principales contingences de la planification dans la petite entreprise, les auteurs décrivent la planification comme etant informelle, sporadique, fermee et effectuée de façon ad hoc, suite à l'apparition d'un problème. Ils mentionnent également qu'elle est en genéral développée par le propriétaire dirigeant seul, se basant sur les conseils d'informateurs qui souvent, possedent moins d'habileté ou d'experience que ce dernier. Toutefois, nous croyons important de mentionner que cette étude a été réalisée auprès d'un echantillon de soixante-sept (67) petites entreprises ayant consulté le Small Business Development Center (SBDC). Ce fait, nous amène à nous interroger quant à la représentativité de l'échantilion puisqu'il s'agit d'entreprises placees dans un contexte particulier.

Dans une autre étude de Robinson et Pearce(1984) sur l'état de la recherche concernant la planification dans les petites entreprises, les auteurs affirment une nouvelle fois que la planification est rarement formalisee, mais ils ajoutent également qu'elle n'est jamais communiqué au-delà d'un petit groupe de personnes et que la recherche d'options est passive et caractérisée par l'acceptation de la premiere alternative attrayante. De plus, ils y décrivent la planification comme étant nonstructurée, irregulière et segmentaire.

Robinson, Logan et Salem(1986) s'intéressant à la planification stratégique et opérationnelle ont constaté que plus de $85 x$ de entreprises étudiées (sur un échantillon de 81 ) ne pratiquaient pas de facon systématique la planification stratégique démontrant une fois de plus la nature intermittente de celle-ci. 
De plus, l'étude de Jones (1982) dont il est fait mention dans la section précédente tend à démontrer que le style de management et d'organisation dans les petites entreprises est informel, venant ainsi appuyer les dires de Robinson (1981 et 1984). Il ajoute egalement que ce style de management permet une adaptation facile au changement et une communication ouverte entre les membres de l'equipe managériale, caracteristique qui a eté identifié par Julien et Marchesnay (1987) également.

Stoner (1983) dans son etude sur la planification dans les petites entreprises manufacturieres ayant entre 2 et 150 employes, nous apporte les informations suivantes. Dans un premier temps, il constate que $21 x$ des entreprises à l'étude préparent des plans a long terme alors que $52 x$ de celles-ci se preoccupent des objectifs a court terme. De plus, il observe que la totalité des entreprises effectuant des plans á long terme développent également des objectifs à court terme et qu'il s'agit en général de plus grandes entreprises.

Shrader, Mulford et Blackburn(1989), quant à eux, ont confirmé l'hypothese a l'effet que la planification operationnelle serait plus utilisee que la planification stratégique dans les petites entreprises. lls ont également démontré que plus l'environnement est incertain, plus les entrepreneurs se préoccupent de planification strategique et opérationnelle.

Par ailleurs, Unni et al. (1981) s'interessant au rôle de la planification stratégique dans les petites entreprises manufacturieres ont démontré que la planification stratégique etait largement utilisée en ce qui a trait aux activités de vente. Ceci leur est apparu paradoxal au fait que peu d'efforts soient faits en cette matiere concernant la publicité et la promotion. 
De plus, Robinson, Logan et Salem(1986) ont constaté que le pourcentage des répondants (détaillants indépendants de l'alimentation) engagés dans des activités de planification opérationnelle concernant chacun des champs fonctionnels à l'étude étaient de $65 \%$ pour le marketing, 56.8x pour les inventaires, $49.4 \%$ pour les activités financieres et 37.0x pour le personnel.

Suite aux résultats obtenus lors d'une étude réalisée par le Small Business Development Center (SBDC) dont nous avons fait mention précedemment, Robinson et Littlejohn(1981) différencient la planification de la petite entreprise de celle rencontrée dans la grande entreprise sur trois (3) dimensions, soit: son caractere informel, son orientation sur le court terme, sa concentration dans les champs fonctionnels et l'utilisation de conseillers externes lbanquiers, avocats, comptables, connaissance en affaires ou consultant en management).

Une des caractéristiques principales de la petite entreprise étant l'omnipresence du propriétaire dirigeant dans la gestion de son entreprise, nous croyons important de présenter certains des eléments qui définissent l'attitude de ce dernier à l'egard de la planification stratégique.

A ce propos, Shuman (1986) affirme que le facteur "temps" constitue le principal obstacle rencontré dans les entreprises tentant de faire de la planification stratégique dans les PME. Il semble toutefois que les dirigeants considerent que la planification pourrait leur permettre de gérer plus efficacement leur temps, d'augmenter la croissance de leur entreprise et d'avoir une meilleure comprehension du marché. De plus, il semble que les dirigeants qui ont preparé un pland'affaires préalablement au lancement de leur entreprise sont plus enclins a utiliser les 
élements majeurs du processus de planification lorsque l'entreprise est en operation.

Unni et al. (1981) ont constaté que l'age et l'éducation du proprietaire dirigeant semblaient liés à la présence de la planification stratégique en ce qui concerne les entreprises minoritaires. En effet, les personnes engagees dans un processus de planification stratégique avaient typiquement une éducation de niveau collégial et étaient agées entre 31 et 35 ans.

Dans le meme ordre d'idée, Jones (1982) dans son étude sur les caracteristiques de la planification dans les petites entreprises lentreprises de service et manufacturesl a distingue les planificateurs des non-planificateurs a l'aide d'une question. Cette derniere demandait aux répondants de décrire l'étendue de leur planification concernant les objectifs et stratégies a court terme comparativement aux objectifs et stratégies a long terme. Ceux ayant obtenu un résultat au-dessus de la médiane étaient classifiés comme des planificateurs. Les résultats de cette étude, ont permis de constater que les planificateurs etaient plus agés et plus scolarisés.

De plus, Jones (1982) a identifié egalement huit( 8$)$ variables qui distinguent les planificateurs des non-planificateurs, soit: l'utilisation d'étude de marché, les prévisions à long terme, l'environnement limité, l'éducation du planificateur, le retour sur l'actif, l'age du planificateur, la participation a la budgetisation du capital et la prévisibilité de l'environnement. Il ajoute que la planification se classerait au deuxieme rang dans les activités journalieres des dirigeants et que l'inhabileté du proprietaire à gérer son temps expliquerait sa sporadicite. 
Dans un autre ordre d'idees, mentionnons que l'approche contingentielle de la planification stratégique devient une préoccupation pour les chercheurs. A cet effet, Robinson, Logan et Salem(1986) affirment qu'il est difficile, voire meme impossible, de generaliser les pratiques manageriales a toutes les situations. Ils ajoutent que certains auteurs suggerent que la taille de l'entreprise serait une variable critique de contingence en ce qui concerne la recherche sur la planification organisationnelle et le développement de théorie.

De plus dans une etude sur la relation entre le stade de developpement, la planification et la performance, Robinson et al.(1984) se réfèrent à l'étude de Vozikis \& Glueck (1980) qui conclut que le stade de développement a une influence importante sur la formalité et sur les conditions de realisation de la planification strategique. lls citent également Hofer (1975) qui affirme que la variable la plus fondamentale dans la détermination d'une stratégie est le stade de vie duproduit (cycle). De plus, ils mentionnent l'etude de Lindsay et Rue (1980) dans laquelle les auteurs concluent que le stade de développement et la taille de l'entreprise sont d'importants facteurs de contingence dans le processus de planification à long terme. Ainsi, Linsdsay et Rue (1980) affirment que le stade de developpement semble jouer un rôle contingent en terme d'intensité de planification, et non pas en terme de procédures.

En résumé, les PME ne semblent pas engagées dans un processus de planification strategique systematique et ce principalement en raison d'un manque de temps. Par contre, la planification operationnelle semble les préccuper davantage, particulierement en ce qui concerne la fonction marketing. 
Nous constatons également que la planification strategique est pratiqué de manière plutôt informelle, sporadique et est orienté vers le court terme. De plus, elle est généralement réalisée par le dirigeant seul qui, toutefois, n'a que trés peu de temps a y consacrer.

Finalement, le stade de developpement exerce une influence sur le degré de formalisme, sur les conditions de réalisation et sur l'intensite de la planification stratégique.

\subsubsection{Impact de la planification dans les PME}

La seconde dimension d'analyse etudie l'impact de la planification stratégique sur la vie future ou sur les performances des petites entreprises. A cet effet, Robinson et Pearce (1984), precise que la plupart des études consultées demontrent que la présence (ou absence) de planification influence la survie éventuelle de la petite entreprise. Ainsi, Mayer \& Golstein (1961) (Voir Robinson, 1984) concluent que les causes majeures de la faillite sont le manque de planification et de prise de décision systématique. L'étude de Wyant (1977) vient également appuyer ces resultats en démontrant que les faillites d'entreprises sont causées par l'inexpérience en management, l'incompétence et le manque de planification. Par ailleurs, Robinson, Pearce et al. (1984) soulignent le fait que plusieurs études ont démontré de facon limité la valeur de la planification strategique pour la petite entreprise. Ainsi, ils mentionnent les études de Woodruff \& Alexander (1958), de Chambers \& Golde (1963), et l'etude de l'Université d'lowa (1963) qui concluent qu'une planification systématique et consciencieuse est plus fréquente dans les entreprises à success. Cependant, ils ajoutent que les conclusions de ces 
études étaient basées sur des interpretations subjectives de leurs observations et non sur l'analyse statistique.

Robinson, Pearce et al. (1984) citent également les études de Robinson (1980)(1982), Trow(1961) et Bracker(1982) qui démontrent avec une évidence limitée que la planification stratégique augmente la performance des petites entreprises mesurée en terme de croissance et de profits.

Robinson et Littlejohn(1981), quant à eux, affirment que la planification est bénéfique à la petite entreprise. En effet leur etude effectuée sur un échantillon de soixante-sept (67) entreprises engagés dans la planification stratégique suite a une consultation avec le Small Business Developpement Center (SBDC) démontre que les ventes ainsi que le nombre d'employés ont augmenté alors que le profit net avant impot calculé en pourcentage des ventes s'est amélioŕ́ de $45 \%$.

Robinson et al. (1984), quant à eux, citent l'étude de Potts (1977) qui démontre que les entreprises qui ont davantage de succés sont plus enclines a faire appel a des services comptables externes pour les supporter dans leurs activités de planification stratégique.

Dans leur étude sur la planification stratégique versus la planification operationnelle, Robinson, Logan et Salem(1986) précisent que les répondants pensent de façon générale que la planification operationnelle contribue davantage au succes de leur entreprise que la planification stratégique. De plus l'étude démontre que les entreprises ayant une moyenne supérieure d'utilisation des activites de planification operationnelle ont un niveau superieur de performance (pourcentage d'augmentation des ventes, pourcentage d'augmentation du retour sur les ventes, 
ventes par employés) comparativement aux autres. Le lien entre la planification strategique et la performance n'a pu etre établi de facon directe. Cependant, les répondants engagés dans la planification stratégique ont une meilleure perception de leurs performances que les répondants qui ne font pas de planification stratégique. Les auteurs indiquent que ce phénomene peut s'expliquer par un biais statistique ou par le fait que les mesures de performance utilisees misent sur le court terme tandis que les performances perçues se concentrent sur le long terme. Finalement, les entreprises mettant l'emphase sur les deux types de planification soit stratégique et operationnelle ont obtenu de meilleures performances sans toutefois surpasser les entreprises engagés dans la planification opérationnelle seulement.

Globalement, Schrader Mulford et Blackburn (1989) ont observé qu'une relation plus ou moins significative existait entre la planification strategique et la performance. Cependant, en ce qui concerne le peu d'entreprises de l'échantillon engagés dans une planification formelle, la relation entre la planification stratégique et la performance semblait plus évidente. De plus, ils ont démontréque la planification opérationnelle, particulierement en ce qui concerne le marché et le budget, influence la performance des petites entreprises.

Robinson et al.(1984) ont également étudié le stade de développement de l'entreprise comme facteur de contingence de la planification strategique. Pour ce faire, ils ont utilisé le modele de Cooper qui identifie trois (3) stades de développement, soit: le demarrage, le "d́but" de croissance et la croissance avancee. Ils concluent que l'amélioration de l'efficacité de la planification stratégique dans la petite entreprise n'est pas contingente avec le stade de développement. En effet, la petite entreprise engagé dans la planification strategique, peu importe 
son stade de développement, améliore son efficacité. lls affirment également qu'un minimum de planification strategique engendre unimpact positif sur la performance.

En conclusion, bien qu'on associe le manque de planification a la faillite, la relation entre la planification strategique et la performance n'est pas encore clairement établie. Il semble toutefois que la planification opérationnelle contribuerait, quant à elle, à l'amélioration des performances des petites entreprises. De plus, l'on constate que l'efficacite de la planification strategique dans les petites entreprises n'est pas contingente au stade de développement.

\subsubsection{Contenu de la planification stratégique dans les PME}

Bien que l'influence entre la planification strategique et la performance ne soit pas encore clairement établie, on observe toutefois une certaine relation entre ces deux (2) variables. Ainsi, il est démontré que l'absence de planification constitue l'une des causes majeures de la faillite. Quant a la planification operationnelle, son influence sur l'amelioration des performances est un fait genéralement reconnu. Ainsi, l'efficacité de la petite entreprise semble líé, en partie, la pratique de la planification. Il faut done s'interroger sur le contenu spécifique de la planification stratégique en contexte de petites entreprises. 


\subsubsection{Contenu des stratégies dans le PME}

Certains auteurs se sont intéresses aux possibilites de classifier les entreprises en fonction de la stratégie choisie. Ainsi, Davig (1986) dans son étude sur les stratégies dans soixante $(60)$ petites entreprises manufacturieres (vetements, fonderies et fabrication de métaux) se réfere a deux (2) types de classifications de stratégies. Il parle d'abord de la classification de Mintzberg qui distingue trois ( 3 ) approches ou styles de stratégies differents, soit l'entrepreneur, le planificateur et l'adapteur sans les définir davantage. Puis, il nous décrit la classification développée par Miles et Snow (1978) a laquel le il se réfère. Miles et Snow (1978) ont identifiéquatre (4) types de stratégies qui ont été testées à plusieurs reprises dans de plus grandes entreprises et que nous présentons à la figure 3. Ainsi, Davig (1986) a démontré que les stratégies de prospecteur et de défendeur obtenaient de meilleures performances (profits) dans les petites entreprises des industries fragmentées comme le vetement, les fonderies et la fabrication du métal. Suite à son étude, il décrit les prospecteurs comme recherchant continuellement de nouveaux produits ou des ameliorations pour les produits courants afin de les rendre plus competitifs tout en maintenant les prix concurrentiels. Quant aux défendeurs, il les décrit comme étant centrés davantage sur le produit que sur le client. L'idée derriére cette approche est de développer un excellent produit et de percer le marché gräce à ce dernier tout en surveillant attentivement les coúts. En général la qualité et les prix sont plus élevés que chez les prospecteurs.

D'autres auteurs ont tenté d'identifier, de maniere plus précise, certains élements relatifs au contenu de la planification stratégique et opérationnelle dans les PME. Ainsi, Robinson et Pearce (1984) dans leur article sur l'etat de la recherche nous résument la situation en ce qui 
Figure 3

\section{Classification des comportements strategiques} selon Miles et Snow (1978)

\begin{tabular}{|c|c|}
\hline DEFENS IF & $\begin{array}{l}\text { L'entreprise s'installe et se maintient } \\
\text { dans une niche relativement stable. }\end{array}$ \\
\hline PROSPECTEUR & $\begin{array}{l}\text { L'entreprise opere de facon typique avec } \\
\text { une definition de marche ou produit } \\
\text { moyennement etendu et, les produits ou } \\
\text { services offerts peuvent varier perio- } \\
\text { diquement. }\end{array}$ \\
\hline ANALYSTE & $\begin{array}{l}\text { L'entreprise s'attend a maintenir une } \\
\text { ligne de produits relativement limitee } \\
\text { et stable tout en tentant de suivre les } \\
\text { nouveaux développements prometteurs dans } \\
\text { l'industrie. }\end{array}$ \\
\hline $\begin{array}{l}\text { REACTIF } \\
\text { (2 types) }\end{array}$ & $\begin{array}{l}\text { L'entreprise démontre une approche moins } \\
\text { consistante que les trois autre types, } \\
\text { une sorte de non-strategie. Elle ne } \\
\text { prend pas de risques avec de nouveaux } \\
\text { produits ou services a moins d'etre } \\
\text { menacé par les competiteurs. }\end{array}$ \\
\hline
\end{tabular}

Inspire de Bamberger (1985)

concerne la recherche sur le contenu de la planification. Ils mentionnent entre autre l'étude de Dess et Davis (1982) qui, à l'aide de l'analyse factorielle, ont identifié trois (3) facteurs fondamentaux qui vont en paralléle avec les stratégies de Porter. Cette recherche a démontré que les entreprises etudiees suivaient une des trois (3) strategies identifiés et que des méthodes specifiques de competitivitéetaient associées à ces stratégies.

Perry (1986) dans son article traite des strategies de croissance (définie en terme de ventes) des PME ayant atteint le stade "confort". II 
definit ce stade comme etant le moment où le traumatisme du demarrage est dépassé et où l'entreprise a atteint un chiffre de ventes suffisant pour assurer sa survie et lui fournir le standard de vie requis ainsi qu'un retour sur l'investissement. Pour réaliser son etude de cas, Perry (1986) a utilisé les quatre (4) stratégies majeures suivantes: la pénétration d'un marché existant avec un produit existant; le developpement d'un nouveau marche pour des produits existants; le développement de nouveaux produits pour des marchés existants; la diversification dans de nouveaux marchés avec de nouveaux produits. II mentionne cependant l'existence de deux (2) autres strategies, soit: devenir son propre fournisseur et devenir son propre distributeur. Suite a l'étude approfondie de trois (3) entreprises australiennes en croissance, Perry émet l'hypothese que les stratégies les plus appropriées pour les entreprises ayant atteint le stade "confort" sont, dans l'ordre: le développement de marchés et le développement de produits. Toutefois, etant donné le caractère exploratoire de cette étude, il mentionne la nécessité d'effectuer d'autres recherches dans le but de valider son hypothèse.

Par ailleurs, Robinson et al.(1984) dans leur recherche sur la relation entre les stades de développement et la planificationstratégique réalisée auprés de cinquante et une (51) entreprises (détaillants et entreprises de servicesl ont découvert qu'au stade 1, la planification stratégique était axée sur l'augmentation de la part de marché (croissance des ventes). Au stade 2, elle concentre ses efforts sur la croissance de l'entreprise (ventes et taille organisationnelle) afin de devenir un compétiteur établi et viable. Tandis qu'au stade 3 , elle met l'emphase sur la stabilisation de la croissance et sur l'augmentation des profits pour assurer la viabilité long terme de l'entreprise en terme de production et de marché. Il semble donc que le centre des efforts de planification varie en fonction du stade de développement. 


\subsubsection{Contenu de la planification stratégique dans les PME}

Stoner (1983), dont nous avons déjà cité l'étude, a obtenu les résultats suivants concernant le contenu des objectifs à court terme: projections des ventes et des profits (94\% des répondants); projections concernant les mouvements de trésorerie (81\%); projections concernant les besoins en capitaux (75\%). Il conclut qu'en général les objectifs court terme sont centrés sur les finances de l'entreprise mais que 19\% des entreprises se préoccupaient également des besoins en ressources et support (tel l'équipement, le matériel et la main-d'oeuvre). Stoner (1983) ajoute que le contenu des plans à long terme ( 3 à 5 ans) des entreprises à l'étude incluait généralement des projections concernant les profits, le taux de retour sur investissement, le point mort, les ventes, le taux de croissance, les coijts et les depenses ainsi que les capacités operationnelles. De plus, il ajoute que $69 \%$ des répondants engagés dans la planification à long terme avaient inclu l'évaluation du point mort dans leurs plans tandis que $46 \%$ d'entre eux $y$ inseraient des pro forma financiers. Il ressort egalement de cette étude que les plus grandes entreprises semblaient plus enclines a preparer des budgets mensuels.

Le rôle du planificateur a également été mis en évidence en ce qui concerne le contenu de la planification strategique et operationnelle. En ce sens, Jones (1982) dans sa recherche sur les caractéristiques de la planification réalisée auprés de soixante-neuf (69) entreprises (entreprises de services et manufacturel a decouvert que les planificateurs se distinguaient des non-planificateurs de la façon suivante:

- Utilisation de la planification a court terme;

- Analyse des ratios; 
- Utilisation d'études de marché dans le but d'innover;

- Recherche d'opportunité d'investissement;

- Participation dans les décisions liées aux produits/services;

- Participation dans les stratégies de croissance.

11 ajoute que les planificateurs semblent plus enclins à faire appel à des consultants pour les aider dans leurs décisions principalement en ce qui concerne la production, la budgétisation et le développenent de stratégies de croissance.

En résumé, nous retenons de ces informations que les entreprises peuvent etre classifiés en fonction des comportements stratégiques adoptés par l'entrepreneur ou le stratege. A cet effet, la classification de Miles et Snow (1978) apparait comme l'une des plus appropriés. Il semble également que le comportement adopté par l'entrepreneur puisse influencer la performance de l'entreprise, dans certains cas.

De maniere générale, les plans à long terme incluent des projections concernant les profits, le retour sur investissement, le point mort, les ventes, le taux de croissance, les coîts et les dépenses ainsi que les capacités operationnelles. Quant aux objectifs à court terme, il se preoccupent des ventes et des profits, des mouvements de tresorerie et des besoins en capitaux. Le stade de développement quant à lui, influence sur l'orientation de la stratégie.

L'utilisation de la planification a court terme, l'analyse des ratios, l'utilisation d'étude de marche, la recherche d'opportunite d'investissement, la participation dans les décisions relatives aux produits/services et la participation dans les strategies de croissance sont, quant à eux, des facteurs qui caractérisent les planificateurs. 


\subsubsection{Processus de planification stratégique dans les PME}

Ayant pris connaissance des principaux faits connus concernant le contenu de la planification stratégique et operationnelle, nous nous sommes interrogés sur la maniere dont les strateges, dans les PME, procédaient pour elaborer leur stratégie.

Shuman et Seeger (1986) dans une recherche effectuée aupres d'un echantillon de deux cent vingt (220) entreprises ont tenté de déterminer les activites faisant partie du processus de planification des petites entreprises. Pour ce faire, ils ont basé leur étude sur le modele proposé par Andrew (1980). Ils ont ainsi découvert que les activités de planification stratégique dans ces entreprises sont generalement orientées sur le court-terme et l'operationnel et qu'elles sont revisees régulièrement. Ils concluent que l'approche typique de planification inclut l'analyse de la competition (60\%), l'identification des besoins de la clientele (74\%), le développement d'un plan détailléd'allocation des ressources $(65 \%)$, l'analyse des forces et faiblesses opérationnelles (76\%), la consideration de plan de contingence (86\%), l'allocation de ressources pour le contrôle et la rétro-action (70\%), et les procedures d'implantation (86x). Ces résultats tendent a démontrer que l'analyse de l'environnement sectoriel se concentre principalement sur la concurrence et à la clientele.

Ils ont observé également que les dirigeants préferent s'impliquer activement dans le processus de planification plutôt que de déléguer cette tache a d'autres membres de l'organisation. De plus, il semble que la sélection des objectifs à court terme soit au centre de leur preoccupation. Par ailleurs, environ $15 \%$ des répondants font appel à d'autres 
ressources qu'ils selectionnent en raison de leur connaissance fonctionnelle plutôt que pour leur expertise en planification.

Specifiquement, les recherches sur le processus de planification stratégique dans les PME sont peu nombreuses, toutefois plusieurs auteurs se sont intéressés à la question et ont développé des modeles thériques de planification stratégique en contexte de PME. Une recension de la littérature nous a permis d'identifier certains de ces modeles de processus de planification stratégique adaptés à la PME (tableau 2 ).

L'analyse des modeles consultés, nous a permis d'identifier plusieurs similitudes entre ces modeles théoriques adaptés a la PME et le modele normatif de Jauch et Glueck (1990).

Premierement, la spécification des objectifs constitue un élement du management stratégique, au sens où l'entendent Jauch et Glueck (1990), qui est présenté de manière à peu prés constante dans l'ensemble des modéles étudiés. De plus, bien que les auteurs n'utilisent pas tous la même terminologie en terme d'analyse et de diagnostic, ils proposent tous une etape d'analyse des environnements interne et externe.

Quant au choix des strategies, nous concluons que tous, a l'exception de Amar et Oliel-Amar (1985), Moyer (1982) ainsi que Schollhammer et Kuriloff (1979), integrent à leur modele les etapes d'élaboration et de choix des options.

En ce qui a trait au processus d'implantation des stratégies ainsi qu'au processus d'evaluation et de contrôle (retroaction), les modeles de Jauch et Glueck (1990), Andrew (1980), Thurston (1984), Vankirk et Noonan (1982), Schol 1 hammer et Kurilloff (1979) considerent de manière plus ou 
Tableau 2

Modèles de planification stratégique adaptés à la PME

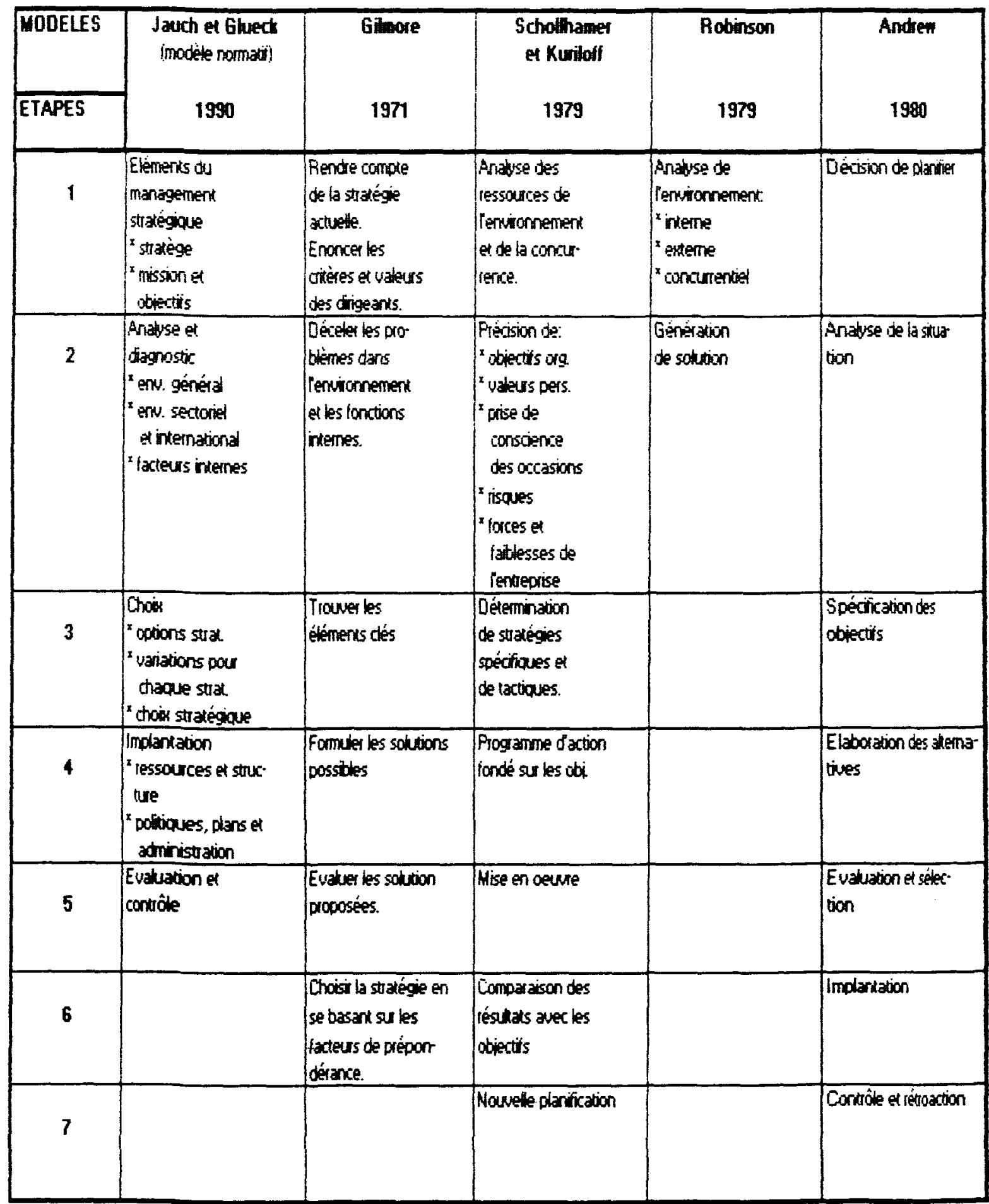


Tableau 2 [suite)

Modèles de planification stratégique adaptés à la PME

\begin{tabular}{|c|c|c|c|c|c|}
\hline MODELES & $\begin{array}{l}\text { Jauch et Ghuect } \\
\text { (modite romati) }\end{array}$ & Nagel & $\begin{array}{l}\text { Van kirt } \\
\text { et Hoonan }\end{array}$ & Moyer & Ragab \\
\hline ETAPES & 1990 & 1981 & 1982 & 1982 & 1983 \\
\hline 1 & $\begin{array}{l}\text { Elements du management } \\
\text { staregique } \\
{ }^{x} \text { statege } \\
{ }^{x} \text { mission et obiectis }\end{array}$ & $\begin{array}{l}\text { Noxet les signass } \\
\text { indiquant que des } \\
\text { ptans sont nétes. } \\
\text { sares. }\end{array}$ & Dein lentreprise & $\begin{array}{l}\text { Horizons de planitar } \\
\text { tion }\end{array}$ & Détiri la mission \\
\hline 2 & 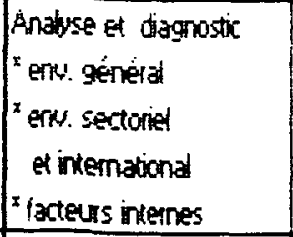 & $\begin{array}{l}\text { Rassembler et } \\
\text { dasser firioma } \\
\text { tion sut fenvion- } \\
\text { nement et su } \\
\text { Penteprise. }\end{array}$ & $\begin{array}{l}\text { Combrende ke } \\
\text { cinat cresent }\end{array}$ & Buts at utiectïs & Choisi une ricte \\
\hline 3 & 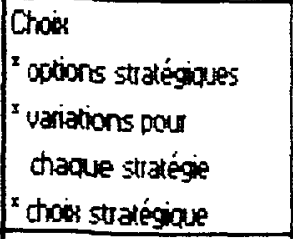 & $\begin{array}{l}\text { Evaluer lo } \\
\text { concurence }\end{array}$ & $\begin{array}{l}\text { Chotsi des } \\
\text { ibjectifs approosies }\end{array}$ & $\begin{array}{l}\text { Euablation des } \\
\text { compétences } \\
\text { distinctives }\end{array}$ & $\begin{array}{l}\text { Idertifies ke polentiel } \\
\text { daction }\end{array}$ \\
\hline 4 & $\begin{array}{l}\text { Implantation } \\
{ }^{x} \text { ressources et structure } \\
\text { " poiniques, plans at } \\
\text { atrinistration }\end{array}$ & 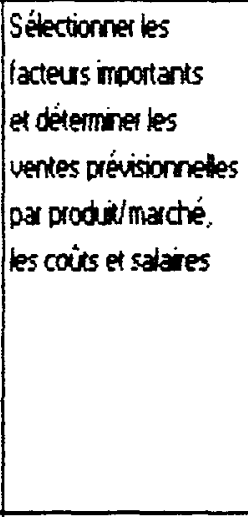 & $\begin{array}{l}\text { Sélectionner les } \\
\text { alematives }\end{array}$ & $\begin{array}{l}\text { Anabse de lenvion } \\
\text { nemenx }\end{array}$ & 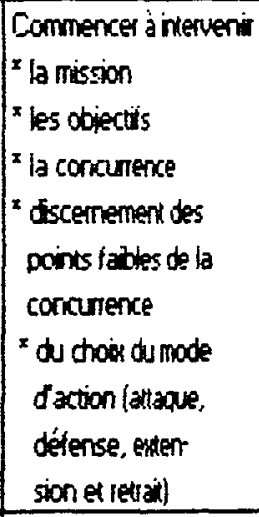 \\
\hline 5 & $\begin{array}{l}\text { Euatuation at } \\
\text { contrôle }\end{array}$ & $\begin{array}{l}\text { Oévelopoer des } \\
\text { posstinés stakég- } \\
\text { ques. }\end{array}$ & $\begin{array}{l}\text { Prépares los olans } \\
\text { tratégiques }\end{array}$ & $\begin{array}{l}\text { Analyser la concas } \\
\text { lence }\end{array}$ & $\begin{array}{l}\text { Dessine le gand } \\
\text { proint stalege de } \\
\text { marchéproduction, } \\
\text { orgarisation, friance }\end{array}$ \\
\hline 6 & & & $\begin{array}{l}\text { Exícuter le olan } \\
\text { hatilement. }\end{array}$ & $\begin{array}{l}\text { Anduse de la } \\
\text { fienciele }\end{array}$ & \\
\hline 7 & & & $\begin{array}{l}\text { Fourri des contồles } \\
\text { sóquats }\end{array}$ & & \\
\hline 8 & & & $\begin{array}{l}\text { Foumin des plans } \\
\text { coningentiels. }\end{array}$ & & \\
\hline
\end{tabular}


Tableau 2 (suite)

Modèles de planification stratégique adaptés à la PME

\begin{tabular}{|c|c|c|c|c|}
\hline MODELES & $\begin{array}{l}\text { Jauch et Gluect } \\
\text { (modste romati) }\end{array}$ & Thuston & Sonfield & Ana \\
\hline ETAPES & 1990 & 1984 & 1984 & 1985 \\
\hline 1 & 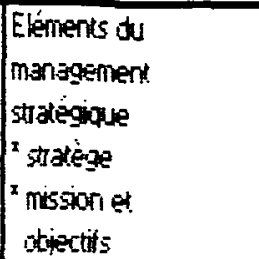 & $\begin{array}{l}\text { Comaitue son entse- } \\
\text { prise }\end{array}$ & $\begin{array}{l}\text { Mise en écidence des } \\
\text { forces de son erroprise }\end{array}$ & Diagnostic \\
\hline 2 & 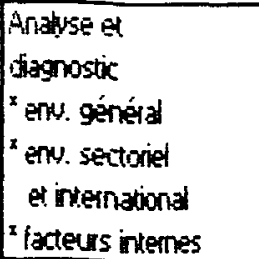 & $\begin{array}{l}\text { Déterminar les } \\
\text { chiectifs }\end{array}$ & $\begin{array}{l}\text { Hise an eviderne des } \\
\text { octasions dewploiter } \\
\text { les forces }\end{array}$ & $\begin{array}{l}\text { Dévelopoement } \\
\text { fobiontifs et doon } \\
\text { stategioues }\end{array}$ \\
\hline 3 & $\begin{array}{l}\text { Choix } \\
\text { "aptions stat. } \\
\text { " variations cour } \\
\text { thaque stak } \\
\text { "dois strágique }\end{array}$ & $\begin{array}{l}\text { Evaluation des } \\
\text { posstités et } \\
\text { defrition du plan }\end{array}$ & $\begin{array}{l}\text { Hise en évidence des } \\
\text { opocortunités ayant des } \\
\text { probabitiés de se } \\
\text { concuétiser }\end{array}$ & $\begin{array}{l}\text { Programme of ations } \\
\text { proritaies }\end{array}$ \\
\hline 4 & $\begin{array}{l}\text { Implantation } \\
\times \text { ressources et stuc- } \\
\text { ture } \\
\text { × poitiones, dans et } \\
\text { atministation }\end{array}$ & $\begin{array}{l}\text { Hise en oeume at } \\
\text { contrôle des écarts }\end{array}$ & $\begin{array}{l}\text { Hise au point de plars } \\
\text { de contingence pour } \\
\text { chaqu opportunité }\end{array}$ & $\begin{array}{l}\text { Adapter la stucture } \\
\text { ogarisationnele }\end{array}$ \\
\hline 5 & $\begin{array}{l}\text { Evaluation et } \\
\text { contuote }\end{array}$ & . & $\begin{array}{l}\text { Altention constanke d } \\
\text { fémergence doccasions }\end{array}$ & implantation \\
\hline 6 & & & $\begin{array}{l}\text { Mise à jour réguese des } \\
\text { plens de contingence }\end{array}$ & Contróle el rétoaction \\
\hline 7 & & & & \\
\hline
\end{tabular}


moins importante ces étapes. Amar et Oliel-Amar (1985) et Ragab (1983), quant à eux, integrent quelques eléments relatifs à l'implantation de la stratégie à leur modele alors que Sonfield (1984) considere la mise à jour des plans parmi les etapes de son processus de planification. Par ailleurs, Moyer (1982), Nagel (1981), Robinson (1979) et Gilmore (1971) ne considerent d'aucune façon l'étape d'implantation de la stratégie à l'interieur de leur modele respectif.

Telle que présentée dans notre analyse des différents processus de planification etudiés, la base des modeles de planification stratégique développés spécifiquement pour la PME ne diffère pas tellement du modele de Jauch et Glueck (1990) qui fut développé dans l'optique de la grande entreprise. Toutefois, certains auteurs identifient des elements caractérisant le processus de planification stratégique dans les petites et moyennes entreprises.

A ce propos, Thurston (1984) insiste sur le fait que l'efficacité de la planification stratégique dans les petites entreprises repose davantage sur la qualité et la pertinence de cette derniére plutót que sur la quantité ou le degré de formalisme rencontré dans la grande entreprise. De plus, Thurston (1984) précise que, dans les petites entreprises, les objectifs personnels du ou des proprietaires se melent souvent aux objectifs de l'entreprise. Il est donc avantageux, selon lui, d'en tenir compte lors de l'élaboration des objectifs de planification. Gilmore (1979) ainsi que Schollhammer et Kuriloff (1979), quant a eux, font ressortir l'importance des valeurs personnelles des dirigeants de la petite entreprise et leur influence sur la planification stratégique. Ces observations s'associent aux specificités de la PME identifiés par Julien et Marchesnay (1987). 
Par allleurs, Amar et 0liel-Amar (1985) soulignent que lors du processus de planification stratégique, l'on doit considerer que dans la petite entreprise, les ressources sont généralement restreintes. De plus, Schollhammer et Kuriloff (1979) mentionnent que la determination des strategies et des tactiques doit permettre d'atteindre les objectifs souhaités et ce, à moindre frais.

Quant a Moyer (1982), il affirme que l'analyse de l'environnement est l'elément qui distingue clairement la planification de la petite entreprise de celle rencontrée dans la grande entreprise. Ceci en raison de l'effet drastique que peuvent avoir les changements environnementaux sur le destin de la petite entreprise. Il ajoute qu'à cette étape, les dirigeants doivent tenter d'identifier un (1) ou deux (2) facteurs environnementaux pouvant potentiellement affecter les opérations de l'entreprise. Par ailleurs, l'avantage le plus important pour la petite entreprise, selon Van Kirk (1982), est probablement l'habileté à choisir des segments qui sont économiquement non-viables pour la grande entreprise.

Nagel (1981), quant à lui, appui son modéle sur l'idée que le dirigeant commence à planifier suite à l'apparition de signes concrets tels qu'une menace, une occasion, une force ou une faiblesse de l'entreprise. Robinson et Littlejohn (1981) viennent appuyer en partie cette affirmation en mentionnant que la planification stratégique dans la petite entreprise fait suite a l'apparition d'un probleme. Cedernier constat, s'inscrit avec la typologie des strategies de Miles et Snow (1978), lorsqu'il parle du réacteur, qui agit en cas de menace. 


\subsection{PLANIFICATION STRATEgIQUE EN PHASE DE PRE-DEMARRAGE}

Tel que démontré précédemment, la littérature abonde concernant la planification stratégique dans les PME. De ces ecrits, l'on retient que la planification strategique est une activité pratiqué de manière informelle, sporadique et qu'elle est orientée vers le court terme. Elle est effectuée par le dirigeant seul, mais ce dernier n'a que très peu de temps à y consacrer. De plus, la planification semble avoir certains effets bénéfiques sur la performance.

L'approche contingentielle a démontré, quant à elle, que le stade de développement influence la pratique de la planification stratégique. Ainsi, le degré de formalisme, les conditions de réalisation et l'intensite de la planification stratégique varient en fonction du stade de développement. Il semble également qu'au premier stade, la planification soit orientée sur l'augmentation de la part de marché. Par ailleurs, certaines études (Fourcade, 1986; Shuman, 1986) concluent que la phase de prédémarrage influence la vie future de l'entreprise.

Suite à toutes ces observations, nous nous sommes intéressés à la planification stratégique dans les entreprises en phase de prédemarrage, sujet à propos duquel la littérature s'avère moins abondante.

\subsubsection{Phase de prédéarrage}

Plusieurs auteurs (Churchill et Lewis, 1983; Fortin, 1986; Cooper, 1979; Gill, 1985) se sont intéressés au développement de l'entreprise. Toutefois, parmi ces derniers, seuls Gill (1985) et Fortin (1986) propo- 
sent des modeles de developpement d'entreprise tenant compte de la phase de prédemarrage.

Le modele de Gill (1985) identifie cinq (5) etapes de développement d'une entreprise soit: decider de partir en affaires, trouver un objectif d'affaires, elaborer et tester le plan d'affaires, demarrer et survivre et finalement, croitre. Les trois (3) premieres etapes s'intéressent donc à la préparation et à la création de l'entreprise tandis que les deux (2) autres s'associent respectivement au demarrage et à la croissance de l'entreprise.

Un autre element intéressant repose sur le fait que ce modele est fondé sur une prémisse voulant que la période de gestation d'une entreprise puisse avoir des consequences fondamentales sur le developpement futur de cette derniere. Notons que cette prémisse est confirmé par l'étude de Fourcade (1986) dont il a etéquestion antérieurement.

De plus, Gill (1985) a identifié trois (3) elements cles du développement d'une entreprise qu'il qualifie d'interactifs et d'interdépendants, soit: les caracteristiques psychologiques et sociales, l'experience de travail et les habiletes de l'entrepreneur; les ressources qu'il peut fournir et enfin, l'idee d'affaires en relation avec les capacites du marché de la supporter. Gill (1985) affirme egalement que lors des premiers stades de développement, une grande emphase est mise sur des criteres d'efficacité liés à la flexibilite, la croissance, l'acquisition des ressources et le developpement d'appui et de marché exterieur.

Se basant sur une analyse de contenu de cas relatifs a des entreprises inscrites au programme S.Y.O.B (Start Your Own Business), Gill 
(1985) décrit les trois (3) premiéres phases du développement d'entreprise de la manière suivante.

La première de ces phases consiste en la décision de partir en affaires. Elle implique une évaluation préliminaire de l'idéd'affaires, des tendances relatives aux ressources et au personnel qui en combinaison commence a fournir les differentes forces relatives aux responsabilités et à la motivation du futur entrepreneur.

La deuxieme de ces phases vise à trouver un objectif ou une idé d'affaires. Ceci s'effectue de diverses manières. Ainsi, quelques uns évaluent systématiquement leurs ressources, habiletés et besoins alors que d'autres, une minorité, ont dés le départ une idée précise de leurs besoins. Finalement, certains n'ont qu'une très vague idée de leur objectif d'affaires et la precise en cours de route. Soulignons, que l'un des principaux facteurs à considérer par l'entreprise lors de l'évaluation des objectifs, que ce soit au stade de prédemarrage ou par la suite, semble etre la connaissance approfondie du marché.

La troisieme phase consiste en l'élaboration et le test du plan d'affaires. Ainsi, les plans d'affaires élaborés par les participants au S.Y.O.B. évaluaient la viabilité de l'objectif d'affaires et présentaient des plans de pénétration en douceur, du marché. De maniére plus précise, l'on y retrouvait une analyse détaillée des ressources nécessaires, l'identification des ressources de support, une analyse de marché ainsi qu'un test de marché. Fait interessant, la plupart des participants éprouvèrent des difficultés à trouver un marché. Des questions fondamentales leurs sont alors apparues, telles: "où et comment trouver de l'information?"; "puis-je falre une meilleure utilisation de mes relations et puis-je les persuader de faire quelques recherches sur le marché pour 
moi?"; "ai-je réellement une prise sur le marché cibléen relation avec les buts de mon entreprise et ai-je développé des stratégies de prix, de distribution et de promotion?". Par ailleurs, les plans varient beaucoup en ce qui concerne leur réalisme. Ils ont tendance à etre davantage optimistes lorsqu'ils sont evalués en prévision de la premiere année en affaires.

Pour sa part, Fortin (1986) dans son modele de développement de l'entreprise identifie quatre (4) stades développement, soit: la preentreprise, le lancement, la croissance et l'expansion-consolidation. Le stade "préentreprise" correspond ici à ce que nous appelons, dans le cadre de cette recherche, la phase de prédemarrage et consiste essentiellement a preparer le plan d'entreprise (Voir Fortin, 1986, p.52). L'auteur qualifie cette étape d'essentielle puisqu'elle assure, en partie, le succes futur du projet. Cette affirmation vient appuyer les observations de Fourcade (1986) dont nous avons fait mention en introduction.

\subsubsection{Plans d'atfaires}

La phase de prédémarrage vise essentiellement la préparation d'un projet d'entreprise et la creation de cette entreprise. Lors de cette phase, le futur entrepreneur tente de déterminer les ressources nécessaires à l'elaboration de son projet et d'en évaluer la faisabilite. Cette démarche peut s'averer relativement difficile d'où l'utilité d'un plan d'affaires. Le plan d'affaires consiste, en quelque sorte, à réaliser un processus de planification stratégique et opérationnel mais ce, a un moment précis de la vie d'une entreprise, soit en période de prédemarrage. De ce fait, le processus s'avere plus complexe puisque 
l'entreprise ne dispose pas encore de systeme d'information établi. Par ailleurs, l'entrepreneur doit tenter de prévoir ce que sera l'entreprise a partir de l'idée d'affaires. Pour ce faire, celui-ci doit rechercher de nombreuses informations qui lui permettront d'effectuer des choix stratégiques judicieux compte tenu des environnements interne et externe auxquels l'entreprise sera confrontée. Ainsi, l'entrepreneur devra identifier les menaces et opportunités de l'environnement et les forces et faiblesses de son projet d'entreprise.

Bref, l'objectif principal de cette démarche consiste à évaluer la viabilité de son projet, a faciliter l'acquisition des ressources et l'obtention de financement et a favoriser, dans une certaine mesure, l'anticipation de difficultés futures (Belley Dussault et Lorrain, 1989). Le plan d'affaires s'avere donc un outil de gestion précieux et de ce fait, sa préparation mérite une attention particulière.

Plusieurs auteurs (Timmons et al.,1985; Mancuso,1985; McClaughl in, 1985; Fortin, 1986; Siegel et al., 1987; Broods et Stevens, 1987; Dell'Aniello, 1987; Gasse et al.,1988; Good, 1989; ONUDI,1978) se sont intéressés au processus de création d'entreprise et ont proposédifférents contenus de plans d'affaires (Voir annexe 2). Belley, Dussault et Lorrain (1989), dans leurs travaux sur le contenu d'un plan d'affaires, ont effectué une analyse de ces différents modeles et nous proposent une synthese du contenu d'un plan d'affaires. Cette structure-synthése a été développée selon une approche systémique et est liée à une démarche de planification stratégique (tableau 3 ).

Le contenu de ce plan d'affaires nous apparait comme l'un des plus complet puisqu'il regroupe l'ensemble des elements composant les propositions de contenu etudiees. Il constitue donc un excellent guide pour la 
Tableau 3

Structure synthèse du contenu d'un plan d'affaires

(Inspire de Belley, Dussault et Lorrain, 1989)

\section{PREMIERE PARTIE}

\begin{tabular}{|c|c|}
\hline SOMMAIRE & TABLE DES MATIERES \\
\hline Llescription sommate oe lentepnse & \\
\hline $\begin{array}{l}\text { Descrotion de lopportunte pousative } \\
\text { et de la stategie d'espioitation }\end{array}$ & \\
\hline $\begin{array}{l}\text { Desciotion du marché usé } \\
\text { el de son potentied }\end{array}$ & \\
\hline Auankage su ta concunence & \\
\hline 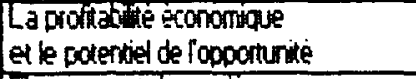 & \\
\hline Financemenx demande & \\
\hline Ditisthon cropose des londs & \\
\hline
\end{tabular}

\section{DEUXIEME PARTIE}

\begin{tabular}{|c|c|c|}
\hline $\begin{array}{l}\text { INDUSTRIE,ORGANISATION, } \\
\text { PRODUIT SISERYICES }\end{array}$ & AKALYSE DE MARCHE & EQUIPE ENTREPRENEURLALE \\
\hline $\begin{array}{l}\text { Anabse de Tindustrie } \\
\text { Definition du secteur } \\
\text { Tendances de coissance } \\
\text { presentes el futures } \\
\text { Anatyse de fenvionnemen gerieral }\end{array}$ & $\begin{array}{l}\text { Identification de la clientele } \\
\text { Nature de la dentéte } \\
\text { Segrnentation de la clenkete }\end{array}$ & Cumichth vitae \\
\hline $\begin{array}{l}\text { Desciption de lorganisation } \\
\text { et de lopportunite } \\
\text { Historique et hauts fats } \\
\text { Nature des produits et des } \\
\text { services offerts } \\
\text { Marches vises el base geographique } \\
\text { Parts de macte } \\
\text { Mages de profits }\end{array}$ & 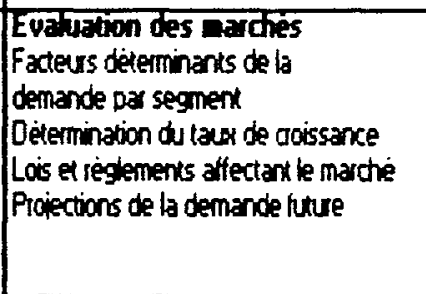 & $\begin{array}{l}\text { Capacte ef atouts } \\
\text { de chacun }\end{array}$ \\
\hline \multirow[t]{4}{*}{ 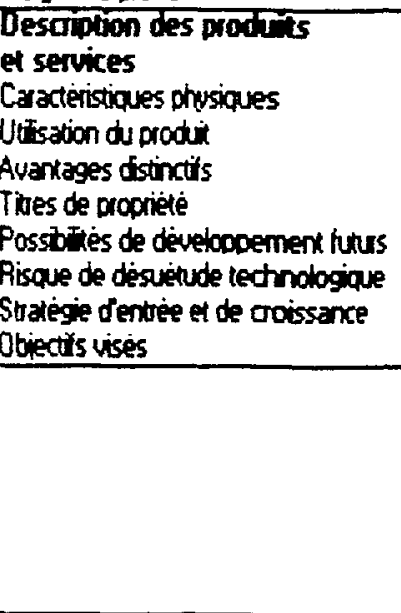 } & 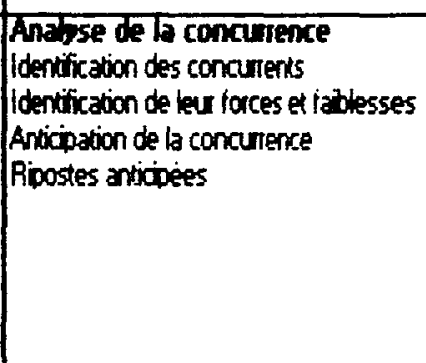 & Reabations pertinentes \\
\hline & base de penetration des narches & $\begin{array}{l}\text { Compowentaite } \\
\text { professionnel }\end{array}$ \\
\hline & $\begin{array}{l}\text { Capacite dusine ef contrankes } \\
\text { tectunologiques }\end{array}$ & Brens petsonnes \\
\hline & $\begin{array}{l}\text { Dêter nation de la } \\
\text { part de parche } \\
\text { Hotwe e lecturowoge }\end{array}$ & \\
\hline
\end{tabular}


Tableau 3 (suite)

Structure synthèse du contenu d'un plan d'affaires

(inspire de Belley et al., 1989)

TROISIEME PARTIE

\begin{tabular}{|c|c|c|c|}
\hline PLAN MAAKETING & $\begin{array}{l}\text { PLAN DE AECHERCHE } \\
\text { ET DEVELDPPEUENT }\end{array}$ & PLAN DES OPERATIONS & $\begin{array}{l}\text { PLAN DES RESSDURCES } \\
\text { HUMANES }\end{array}$ \\
\hline $\begin{array}{l}\text { Stratege generale } \\
\text { Gnmert gt quand les } \\
\text { chentetes seront attenkes }\end{array}$ & stakut de developpewent & 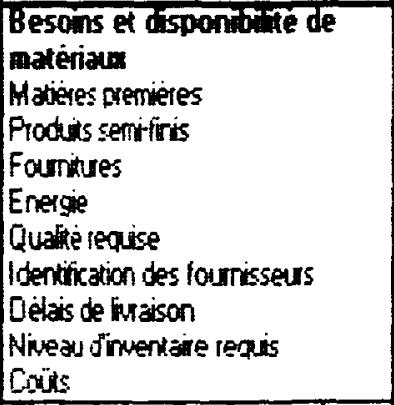 & 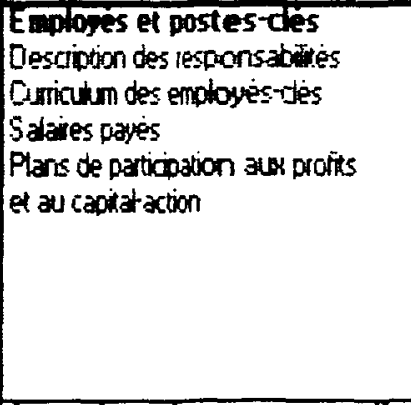 \\
\hline $\begin{array}{l}\text { Strategie de produl } \\
\text { Force du produt erolote }\end{array}$ & Elapes à franth & $\begin{array}{l}\text { Chois de locatsation el ste } \\
\text { Justifiction du choin du site } \\
\text { Auarkage el inconverients } \\
\text { us criteres de choin }\end{array}$ & $\begin{array}{l}\text { Autes investissediss non-actís } \\
\text { Identificiton } \\
\text { Impotance de la particioation }\end{array}$ \\
\hline $\begin{array}{l}\text { Strategie de pris } \\
\text { Base de detemination } \\
\text { Comoarason aver la } \\
\text { concunence } \\
\text { Justification des exats } \\
\text { et des impacts }\end{array}$ & $\begin{array}{l}\text { Otificules et lisques } \\
\text { anticipes }\end{array}$ & $\begin{array}{l}\text { Descriotion de la tectinologie } \\
\text { utisice } \\
\text { Desciption } \\
\text { Temes dapropriation }\end{array}$ & $\begin{array}{l}\text { Consel dad anistration } \\
\text { Identication des membres } \\
\text { Alouts des membres pour } \\
\text { Pentreprise }\end{array}$ \\
\hline 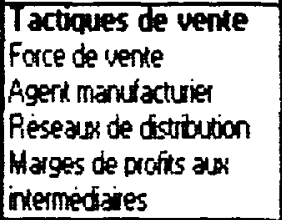 & $\begin{array}{l}\text { Plans futurs de } \\
\text { developpenent }\end{array}$ & $\begin{array}{l}\text { A cenaginen's et equipenents } \\
\text { requis } \\
\text { Liste } \\
\text { Coüs }\end{array}$ & $\begin{array}{l}\text { Conseticis enternes } \\
\text { Auocats } \\
\text { Complatles } \\
\text { Conselers en gestion } \\
\text { Agence de puticite } \\
\text { Autes }\end{array}$ \\
\hline 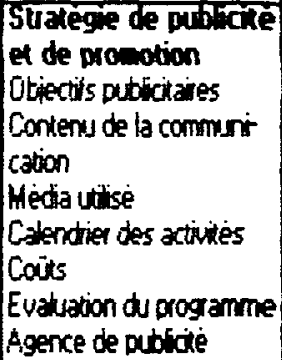 & Equipe de recherche & 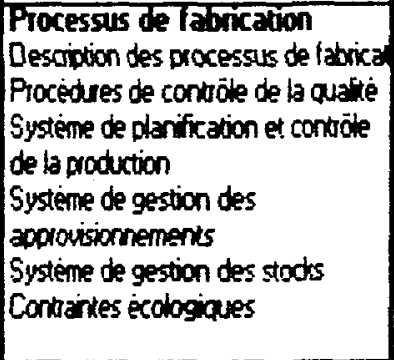 & $\begin{array}{l}\text { Uesciption des drois el } \\
\text { devins des actionnaides }\end{array}$ \\
\hline $\begin{array}{l}\text { Promotions auk points } \\
\text { de ventes } \\
\text { Pubicite gatule }\end{array}$ & & \multirow{4}{*}{ 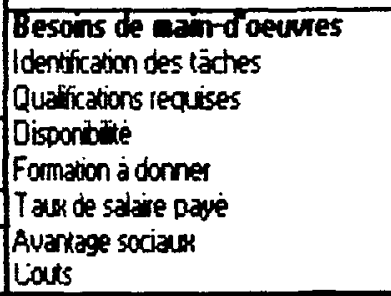 } & \\
\hline $\begin{array}{l}\text { Powique de service } \\
\text { apres-vente }\end{array}$ & $\begin{array}{l}\text { Entente aver organtsines } \\
\text { extèrieu }\end{array}$ & & \\
\hline Powique de garante & Approbacions requises & & \\
\hline $\begin{array}{l}\text { Uelerination des } \\
\text { couts de matreting }\end{array}$ & Couts de Rtu & & \\
\hline
\end{tabular}


Tableau 3 (suite)

Structure synthese du contenu d'un plan d'affaires (Inspirè de Belley et al., 1989)

TROISIEME PARTIE (SUITE)

CALENDRIER DE REALISATION ET LE PLAN DE GESTION DES RISQUES

Le calendrier de reatsation

lidentificaion des etapes impotantes

Elabli les intearijets

Elspes suiettes j potiemes

Flans de courdingerce

Plan de gestion des risques

identibation des risques potentiets

Mojens dour les suter

Flans de contingence

Scenaros optmate et reamate

PLAN OES RESSOURCES FINANCIERES

besons inanciers

Etal des iesulats previsionnets

budget de caisse

brian d ouverturel bians previsionnets

Cacul du point-mort

Anabse des ratios financiess

Caculs de ientabte du protet

Sources de financewent rectierches

QUATRIEME PARTIE

\begin{tabular}{|c|}
\hline $\begin{array}{l}\text { PROPOSITION DE } \\
\text { L'ORGANISATION }\end{array}$ \\
\hline 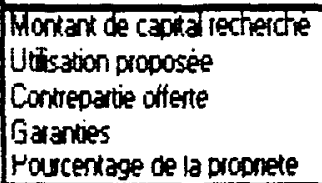 \\
\hline
\end{tabular}

CINQUIEME PARTIE

ANNEXES 
réalisation d'un plan d'affaires. Cependant, tel que mentionnépar les auteurs (Belley, Dussault et Lorrain, 1989) chaque projet d'entreprise possede ses particularités et de ce fait, certains ajustements devront etre falts au modele proposé.

\subsubsection{Recherches sur les entreprlses en phase de prédémarrage}

Le contenu du plan d'affaires tel que proposé par les auteurs constitue en fait une démarche idéale afin de structurer formellement un projet d'entreprise (Belley, Dussault et Lorrain, 1989). La réalité telle que vécue par les entrepreneurs peut parfois s'avérer différente.

Peu d'études spécifiques se sont intéressées aux démarches et aux comportements empruntés par les entrepreneurs afin de structurer leur projet d'entreprise. En fait, parmi la littérature consultée, seuls Lorrain (1990) et Lorrain et Raymond (1988) ont étudié quelques aspects de cette problematique. D'une part, l'etude de Lorrain (1990) avait pour objectif d'établir le profil des jeunes entrepreneurs du Quebec, le cheminement parcouru par ceux-ci lors de l'elaboration de leur projet d'entreprise, le profil organisationnel de leur entreprise ainsi que la réalité d'etre propriétaire-dirigeant pour ces jeunes entrepreneurs québécois. D'autre part, l'objectif poursuivi par Lorrain et Raymond (1988) consistait à établir le profil socio-demographique des propriétaires-dirigeants de nouvelles entreprises et le profil organisationnel de leur entreprise. Les résultats de ces deux recherches nous ont permis d'observer certains faits intéressants. 
Premierement, ces auteurs identifient quelques unes des caracteristiques personnelles et psychologiques rencontrees chez les entrepreneurs. Ainsi, Lorrain (1990) a démontré que les jeunes entrepreneurs (moins de 30 ans) sont, en général, agés entre 25 et 30 ans, qu'ils n'ont pas d'enfants et qu'ils possedent, pour la plupart, au moins un diplóme d'études secondaires avec une specialisation en technique et métier. Ils ont acquis des connaissances et habiletes techniques dans plusieurs cas $(50,5 \%)$ et ils bénéficient d'une expérience sur le marché du travail de 5,4 année en moyenne. Fait intéressant, près de 62 \% des jeunes avaient travaille dans le secteur d'activité de leur entreprise, alors que seulement $23,9 \times$ d'entre eux possédaient une expérience d'affaires. Plus de $70 \%$ de ces jeunes ont travaillé dans des entreprises de 50 employés ou moins (PME). De plus, la famille semble exercer une influence sur les jeunes entrepreneurs puisque plus de $44 \%$ d'entre eux ont un ou plusieurs membres de leur famille en affaires (principalement le pere).

Parallelement, Lorrain et Raymond (1988) ont demontré par leur recherche, que les entrepreneurs québécois sont agés de 31 ans et plus pour la majorité, qu'ils ont 1,5 enfants en moyenne et qu'ils possedent, pour la plupart, au moins un diplôme d'études secondaires. Ces derniers avaient une expérience du secteur d'activité de leur entreprise de 6,7 années en moyenne. Par ailleurs, plus de $35 x$ des répondants ont un parent en affaires et il s'agit principalement d'un frére $(34,5$ x) ou du père $(24,7$ x). Finalement, Lorrain et Raymond (1988) ainsi que Lorrain (1990) constatent, tous les deux, que le besoin d'autonomie constitue la principale motivation des entrepreneurs. Précisons de plus, que plusieurs des faits mentionnés ci-haut s'associent aux dires de Garnier (1986) et Belley (1987), en particulier en ce qui concerne l'experience sur le marché du travail et le contexte familial. 
Concernant les démarches effectuees avant la création de l'entreprise, on constate certains faits intéressants. D'abord, les jeunes entrepreneurs québécois réfléchissent pendant 18 mois en moyenne avant de décider de se partir en affaires (Lorrain, 1990). Quant aux entrepreneurs, en général, ils s'accordent un temps de réflexion de plus de deux (2) ans en moyenne avant de démarrer leur entreprise alors que la mise sur pied de cette dernière nécessite en moyenne 5 mois (Lorrain et Raymond, 1988).

Pour les jeunes entrepreneurs le temps moyen requis pour trouver l'idée d'affaires est de 8,6 mois. Cette idée provient généralement de plusieurs sources dont l'experience de travail $(51,5 \times)$ et les discussions avec l'entourage $(33,2 x)$ ou les gens d'affaires $(23,2 x)$. Dans la majorité des cas $(79,5 \quad$ x), elle est identifiée avant meme que la décision de partir en affaires soit prise (Lorrain, 1990). Quant au temps consacré par les jeunes entrepreneurs à l'élaboration du projet, il est de 8,5 mois en moyenne dont 4,5 mois servent a l'elaboration du plan d'affaires et 4 mois à sa réalisation.

L'élaboration du plan d'affaires inclut les étapes de planification financière ( 31 jours), de planification marketing $(23$ jours), de planification juridique et fiscale ( 20 jours), de montage des operations, de planification des ressources physiques et humaines ( 19 jours) et de planification de la localisation (16 jours).

La réalisation du plan d'affaires, quant à elle, comprend les activités d'aménagement du local (29 jours), de négociation du financement (20 jours), de négociation pour l'acquisition des ressources physiques ( 20 jours), de négociation des ententes avec les futurs clients 
120 jours) et finalement, de préparation des ententes, contrats légaux et des différents formulaires requis ( 17 jours) (Lorrain, 1990).

L'élaboration d'un projet d'entreprise necessite de l'aide et du support. Lorrain (1990) constate que les jeunes entrepreneurs se tournent generalement vers la famille $(82 x)$ et ce, souvent en vue d'obtenir un support moral et emotionnel $(57,9 \times)$. Par contre, la plupart d'entre eux consultent les comptables $(50,8 \times)$, les avocats et notaires $(43,2 \times)$, les gens d'affaires $(39,1$ x), les fournisseurs $(37,6$ x) et les fonctionnaires gouvernementaux $(35,2 x)$ pour obtenir de l'information ou des conseils en affaires. Quant aux questions de financement, ils s'adressent généralement au personnel des institutions financières $(72,1 \mathrm{x})$.

Lorsqu'on les interroge sur les principaux problemes rencontrés lors de la réalisaton de leur projet, les jeunes mentionnent, par ordre d'importance: la lenteur et la paperasserie gouvernementale, l'obtention du financement, la réalisation du plan d'affaires et la planification du projet, le manque d'experience en affaires, les prix et les conditions de credits des fournisseurs et l'obtention d'une clientele à l'avance (Lorrain, 1990).

Concernant le financement du projet, il semble que plus de $57 \times$ des jeunes presentaient un bilan personnel positif avant de se lancer en affaires. Par ailleurs, le capital moyen pour creer l'entreprise se situait a $41128 \%$, cependant environ $50 \times$ des jeunes ont démarré leur entreprise avec un capital de 20000 ou moins (Lorrain, 1990). Dans l'étude de Lorrain et Raymond (1988), le capital investi par les entrepreneurs pour lancer leur entreprise est en moyenne de 22502 \$. Les principales sources de financement des jeunes sont par ordre d'importance: le programme "Jeunes promoteurs" $(24,7$ x), les emprunts bancaires 
sur garantie personnelle $(17,1 \times)$, l'argent personnel des jeunes entrepreneurs $(15,5$ \%) et les prets commerciaux $(10,1$ \%) (Lorrain, 1990). Fait intéressant, Lorrain (1990) souligne que plusieurs jeunes entrepreneurs $(38.8$ \%) considerent que le montant de capital initial investi est insuffisant et qu'en moyenne, une somme de 26515 serait requise pour combler cette lacune.

En résumé, ces études nous apportent des informations interessantes sur la phase de prédémarrage des entreprises et, plus particuliérement, sur les caractéristiques personnelles de l'entrepreneur, le temps de réflexion précédant le lancement de l'entreprise, les sources de l'idée d'affaires, le temps consacré à l'elaboration du projet d'entreprise, les personnes ressources consultées, les problemes rencontrés et le financement de la future entreprise. Cependant, elles ne nous fournissent que tress peu d'informations sur le processus de planification stratégique suivi et sur l'ensemble des actions réalisees par l'entrepreneur lors de la phase de prédémarrage. 
CHAP ITRE II

OBJECTIFS ET HYPOTHESES DE RECHERCHE 
CHAPITRE II

\section{OBJECTIFS ET HYPOTHESES DE RECHERCHE}

L'analyse de la littérature consultée nous apporte de nombreuses informations sur la planification stratégique dans les PME. D'une part, nous observons que la majorité des modeles normatifs de planification stratégique consultés ont été développés dans une perspective de grande organisation. Par ailleurs, des recherches effectuees aupres de PME nous démontrent toute la specificité de la planification stratégique dans ce type d'organisation, de meme que la présence de facteurs de contingence pouvant influencer cette activité managériale tels, le stade de développement. Toutefois, la littérature s'intéressant a la planification stratégique dans les entreprises en phase de prédemarrage s'avère moins abondante et traite principalement de l'elaboration d'un plan d'affaires.

Ainsi, cette recherche vise à ameliorer les connaissances sur la planification strategique dans les entreprises en phase de prédemarrage. Plus précisement, l'objectif général poursuivi dans cette etude se traduit de la façon suivante:

ldentifier et évaluer les comportements de planification stratégique des entrepreneurs lors de la phase de prédémarrage d'une entreprise.

Lors de cette etude descriptive, les differentes données recueill ies permettront de déterminer le profil général des entrepreneurs rencontrés et d'apporter certaines précisions concernant leurs comportements de planification stratégique en phase de prédemarrage. Dans cette perspec- 
tive, les hypotheses formulees porteront principalement sur les comportements de planification stratégique liés à l'analyse des environnements général, sectoriel et interne. Ainsi, les hypothèses retenues sont les suivantes:

Hypothese 1:

Lors de la phase de prédémarrage des entreprises de services du Saguenay-Lac-St-Jean, les entrepreneurs analysent à des degres significativement différents les environnements interne, sectoriel et général.

Hypothèse 2:

Lors de la phase de prédemarrage des entreprises de services du Saguenay-Lac-St-Jean, les entrepreneurs analysent à des degrés significativement différents les divers facteurs de l'environnement interne.

\section{Hypothesse 3:}

Lors de la phase de prédemarrage des entreprises de services du Saguenay-Lac-St-Jean, les entrepreneurs analysent à des degrés significativement différents les divers facteurs de l'environnement sectoriel. 


\section{Hypothese 4:}

Lors de la phase de predemarrage des entreprises de services du Saguenay-Lac-St-Jean, les entrepreneurs analysent à des degrés significativement différents les divers facteurs de l'environnement genéral. 
CHAPITRE III

METHODOLOGIE 
CHAPITRE III

\section{METHODOLOGIE}

Ce chapitre décrit de manière détaillée les moyens et les techniques utilisés afin de recueillir l'information necessaire à la vérification des hypotheses de recherche.

Précisons dans un premier temps, qu'il s'agit d'une recherche fondamentale descriptive (Gauthier, 1986) puisqu'elle vise a identifier les comportements de planification strategique des entrepreneurs en phase de prédémarrage.

\subsection{POPULATION}

La population à l'átude se compose de propriétaires d'entreprises de services dont la firme est en operation depuis moins d'un an et dont la place d'affaires se situe sur le territoire du Saguenay-Lac-St-Jean. Les entreprises issues d'un rachat, d'un legs ou de l'achat d'une franchise ont été exclues de notre échantillon puisque dans ces circonstances, les démarches de l'entrepreneur sont généralement facilitées. De plus, considérant que certaines entreprises ne figurent pas sur les 1 istes et répertoires fournis, nous n'avons pu déterminer avec exactitude la taille de cette population. 


\subsubsection{Choix des entreprises de services}

Nous avons choisi d'effectuer notre étude auprès des entreprises de services en raison de l'augmentation considerable du nombre de petites entreprises oeuvrant dans le secteur des services. Il semble, en effet, que ce phénomene soit dij principalement au fait que le lancement d'une entreprise dans ce secteur d'activités exige relativement peu de capital. De plus, la proximité de ces entreprises avec le marché favorise leur compétitivité dans plusieurs activités de services (Julien et Marchesnay, 1987). Tel que mentionné par Julien et Marchesnay (1987, p. 118): "Le poids economique croissant de ces petites entreprises de services (P.E.S.) et leurs spécificités en font indubitablement un champ d'analyse interessant et prometteur, a divers niveaux de preoccupation."

\subsection{TECHNIQUE D'ECHANTILLONNAGE}

Afin de constituer notre echantillon, nous avons contacté les commissaires industriels du Saguenay-Lac-St-Jean qui nous ont fourni les répertoires et listes des nouvelles entreprises de services situees sur leur territoire respectif. Par la suite, nous avons tenté de joindre par teléphone les propriétaires d'entreprises de services du SaguenayLac-St-Jean ayant moins d'un an d'existence. Suite au premier appel téléphonique, 60x des proprietaires-dirigeants d'entreprises contactés ont accepté de participer à l'etude. Toutefois, 5\% d'entre eux se sont désistés lors d'un deuxieme contact télephonique et un autre $5 \%$ ne se sont pas présentés aux rencontres prévues. Bref, seulement $50 \%$ des personnes contactées ont réllement participé à cette etude, pour un total de trente-cinq (35) repondants. Parmi les questionnaires complétés, trois (3) ont eté rejetes ayant éte jugés non-valides. L'echantillon final se com- 
pose donc de trente-deux (32) proprietaires d'entreprises de services dont la majorité sont en operation depuis moins d'un an. Précisons à nouveau, que nous avons exclu de notre echantillon, les entreprises issues d'un rachat, d'un legs ou de l'achat d'une franchise puisque nous désirons connaitre les comportements des entrepreneurs en phase de predemarrage et que ce type d'entreprises réduit au minimum ou du moins facilite les démarches relatives à cette période de vie de l'entreprise. Ainsi, la méthode d'echantillonnage utilisee pour constituer notre échantillon correspond à une technique d'echantillonnage non-probabiliste et volontaire, telle que décrite par Gauthier (1986).

Cette méthode d'echantillonnage nous semble la plus appropriée compte tenu de la nature meme de l'étude et des moyens techniques et financiers dont nous disposions. En effet, la période visée par l'étude, soit le prédemarrage, exige du participant un minimum de réflexion dans le but de se remémorer les demarches effectués lors de cette phase de vie de l'entreprise. C'est pourquoi, la nature volontaire de la participation revet une importance particuliere afin de $s^{\prime a s s u r e r ~ q u e ~ l e ~ r e ́ p o n d a n t ~ a c-~}$ corde un minimum d'intéret à l'étude, qu'il se souvienne des actions posees lors de la préparation de son projet d'entreprise et ainsi éviter qu'il ne rationnalise l'ensemble ses réponses.

\subsection{INSTRURENT DE MESURE}

Considérant la quantité et la qualitédes informations que nous désirions recueillir, nous avons opté pour l'élaboration d'un questionnaire specifique comme instrument de mesure. Ce questionnaire se compose majoritairement de questions fermees permettant d'évaluer les comportements de planification stratégique des entrepreneurs et ce, à l'aide 
d'une echelle de type Likert en cinq points. La verification de ces echelles de mesure par un test alpha de Crownback demontre leur consistance interne. Afind augmenter la validité de notre instrument de mesure, nous avons également adapté et utilisé certaines questions développees dans le cadre de recherches anterieures (Lorrain et Raymond, 1988; Lorrain,1990). Ainsi, les questions concernant les caractéristiques de l'entrepreneur, les sources d'information et les personnes ressources consultées ont été, pour la plupart, validées lors d'études antérieures.

Le questionnaire comporte trois (3) sections dont les détails vous sont présentés au tableau (4). La premiere section du questionnaire fournit des informations générales sur l'entreprise concernée et est composée de seize (16) énoncés. La deuxieme section, quant à elle, constitue le coeur de cette étude puisqu'elle evalue les comportements de planification stratégique adoptés par les nouveaux entrepreneurs lors de la phase de prédemarrage. L'ordre de présentation des questions refere au modele de planification stratégique de Jauch et Glueck (1990). Ainsi, nous mesurons dans un premier temps, le degré de précision de la mission et des objectifs ainsi que la connaissance du secteur d'activités. Par la suite, nous évaluons le degré d'analyse des différents facteurs 1 iés aux environnements général, sectoriel et interne. Nous vérifions ensuite l'importance et l'intéret des sources d'information et des personnes ressources consultées lors de la phase de predémarrage. Finalement, nous cherchons a identifier le type de stratégie adoptée par ces nouveaux entrepreneurs. Cette section se compose d'un total de cent soixante dixneuf (179) enoncés. La troisieme et dernière section de notre outil de mesure nous fournit, quant a elle, des informations concernant les caractéristiques personnelles de l'entrepreneur. Cette section compte treize (13) énoncés. 
Tableau 4

Contenu du questionnaire

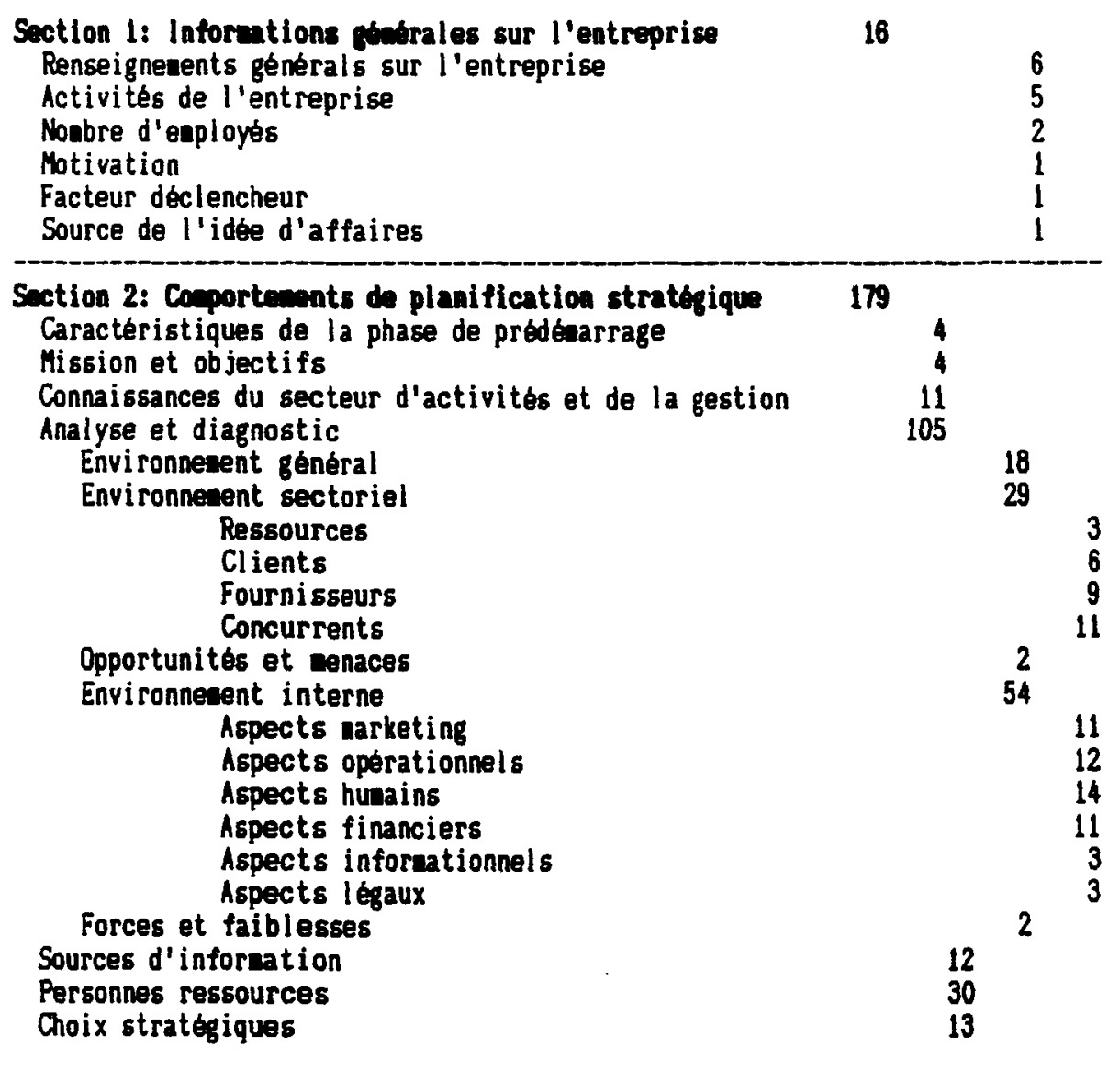

Section 3: Caractoristiques personnol les de l'entrepreneur 13 Caractéristiques générales Caractéristiques spécifiques Fornation et experience 
Afin de verifier la validité de notre instrument de mesure, nous l'avons présenté à un groupe de professeurs en gestion qui en ont fait l'analyse et nous ont suggéré quelques modifications. Par la suite, nous l'avons soumis à cinq (5) entrepreneurs répondant aux critères de l'étude, afin d'évaluer leur compréhension de chaque énoncé et de vérifier la pertinence des questions posées. Nous avons ainsi obtenu le questionnaire final tel que présenté à l'annexe (1).

\subsection{PROCEDURE D' EXPERIMENTATION}

La procedure expérimentale se compose essentiellement de cinq (5) etapes. A la premiere etape nous avons communique avec les commissaires industriels de chacun des secteurs à l'étude (Jonquiere, Chicoutimi, La Baie, Dolbeau et Alma) afin d'obtenir les listes des nouvelles entreprises de services crées au Saguenay-Lac-St-Jean. La deuxieme étape consistait a contacter, a partir des listes obtenues, les entrepreneurs répondant aux criteres d'echantillonnage pour les inviter à participer à cette étude. A la troisieme etape, nous avons réalisédes entrevues de groupe ( 3 a 8 personnes) lors de laquelle les participants devaient completer un questionnaire. Pour assurer une meilleure compréhension, un interviewer présentait les directives générales puis, lisait chacune des questions en fournissant des explications supplémentaires au besoin. A la quatrieme étape, nous avons procédé a la vérification et a la codification des questionnaires complétés. Finalement, à la cinquième étape, nous avons procédé à l'analyse des résultats. 


\subsection{METHODE D'ANALYSE}

Les données ainsi recueillies et codifiees ont été traitees sur un système informatique à l'aide du logiciel SPSS-X (Statistical Package for Social Science).

Dans un premier temps, nous avons analysé les données descriptives de l'étude en utilisant des statistiques, telles: la fréquence, la moyenne, la médiane, le mode, l'écart-type, le minimum et le maximum. Ces statistiques nous ont permis de présenter certaines caractéristiques générales de l'échantillon. Par la suite, nous avons procédé à la vérification des hypotheses a l'aide de statistiques specifiques et appropriees. Ainsi, pour verifier les hypotheses formulees dans le cadre de cette recherche, nous avons utilisé les Tests $T$ de Student qui permettent de vérifier si l'écart entre deux moyennes est significativement différent. 


\section{CHAPITRE IV}

PRESENTATION DES RESULTATS 
CHAPITRE IV

\section{PRESENTATION DES RESULTATS}

Ce chapitre, composé de cinq (5) parties, présente les résultats obtenus aupres d'un echantillon de trente-deux (32) proprietaires-dirigeants de nouvelles entreprises de services du Saguenay-Lac-St-Jean. La premiere de ces parties expose les caracteristiques de l'échantillon. La seconde partie présente les caractéristiques des entreprises echantillonnées tandis que la troisième partie décrit les caractéristiques des entrepreneurs intérroges. La quatrieme partie, quant a elle, présente l'ensemble des résultats liés à la phase de prédemarrage, soit: les caractéristiques générales de la phase de prédemarrage; les connaissances de l'entrepreneur concernant le secteur d'activité et la gestion de l'entreprise; les comportements de gestion des entrepreneurs liés à l'analyse des environnements géneral, sectoriel et interne; les sources d'information consultées; les personnes ressources contactées; et finalement les choix stratégiques privilégies par l'entrepreneur. La cinquieme et derniere partie expose les résultats obtenus à l'aide des tests $T$ qui serviront à la vérification des hypothèses de recherche.

\subsection{CARACTERISTIQUES DE L'ECHANTILLON}

\subsubsection{Repartition des entreprises par ville}

La répartition des entreprises par ville, présentee au tableau 5 , indique que $37,5 \times$ des entreprises à l'étude sont situés à Chicoutimi, 


\section{Tableau 5}

$$
\begin{gathered}
\text { Répartition des entreprises de services } \\
\text { echantillonnés par ville }
\end{gathered}
$$

\begin{tabular}{lrr}
\hline Villes & N & $\boldsymbol{X}$ \\
\hline & 12 & 37,5 \\
Chicoutimi & 10 & 31,3 \\
Jonquiere & 5 & 15,6 \\
Alma & 1 & 3,1 \\
St-Felicien & 4 & 12,5 \\
Dolbeau & & \\
\hline
\end{tabular}

$31,3 \%$ à Jonquière, $15,6 \%$ à Alma, 3,1 $\times$ à St-Félicien et $12,5 \%$ à Dolbeau. Ainsi, $68,8 \%$ des entreprises de l'echantillon operent sur le territoire du Saguenay et 31,2 \% sur le territoire du Lac St-Jean. Rappelons que l'echantillon total de l'étude se compose de 32 entreprises de services du Saguenay-Lac-St-Jean.

\subsubsection{Repartition des entreprises par secteur d'activites}

La classification de Lovelock (1983) a été utilisée afin de répartir les entreprises echantillonnees par secteur d'activites (Tableau 6). Cette classification divise les entreprises de services selon quatre (4) secteurs en fonction de la nature de l'acte de service et du benéficiaire direct du service. 


\title{
Tableau 6
}

Repartition des entreprises de services dchantillonnés par secteur d'activités

\begin{tabular}{lrc}
\hline Secteurs d'activites & N & X \\
\hline & & \\
Actions tangibles/personnes & 5 & 15,6 \\
Actions tangibles/choses & 14 & 43,8 \\
Actions intangibles/personnes & 7 & 21,9 \\
Actions intangibles/choses & 5 & 15,6
\end{tabular}

\begin{abstract}
Ainsi, le premier secteur "Actions tangibles/personnes" correspond aux services destinés aux corps des personnes lsalon de beaute, restaurant,...l; le deuxieme secteur "Actions tangibles/choses" correspond aux services destinés à des biens et autres possessions physiques lentretien et réparation industriel, soins vétérinaires, ...); le troisieme secteur "Actions intangibles/personnes" correspond aux services destinés à l'esprit des personnes leducation, theatre, ...) et finalement, le quatrieme secteur "Actions intangibles/choses" correspond aux services destines á des possessions intangibles (banques, assurances, ...). Les résultats obtenus suite a cette classification (tableau 6) indiquent que la plupart des entreprises de notre echantillon $(43,8 \%)$ appartiennent au secteur "Actions tangibles/choses". De plus, 21,9x des entreprises operent dans le secteur "Actions intangibles/personnes" et pour les secteurs "Actions tangibles/personnes" et "Actions intangibles/choses" on obtient la meme proportion soit, $15,6 \%$.
\end{abstract}




\subsection{CARACTERISTIQUES DES ENTREPRISES}

\subsubsection{Caractéristiques générales des entreprises}

Les résultats présentés au tableau 7, décrivent les caractéristiques générales des entreprises de services echantillonnées. Ainsi, notre echantillon se compose de nouvelles entreprises de services du SaguenayLac-St-Jean dont l'äge moyen etait, au moment de l'étude, de 7,07 mois (ecart-type de 4,30). Soulignons que l'un des principaux critères d'échantillonnage de l'étude voulait que ces entreprises aient moins d'un an d'existence. Toutefois, nous observons que trois (3) des entreprises étudiées avaient débuté leurs operations depuis plus d'un an $(14$ ou 15 moisl. Malgré ce fait, ces entreprises ont été inclues dans notre échantillon puisque nous considérons qu'il s'agit d'un écart relativement petit. De plus, vingt-quatre $(24)$ des entreprises de notre échantilion étaient enregistrées ou incorporés depuis moins d'un (1) an et sept (7) depuis plus de douze (12) mois. Il s'agit majoritairement d'entreprises à propriétaire unique $(68,8$ \%) et $68,8 *$ des entrepreneurs intérrogés affirment n'avoir aucun associé. Par ailleurs, 56,3 * des entrepreneurs n'ont aucun membre de leur famille au sein de leur entreprise.

\subsubsection{Caractéristiques 1 iées aux activités de l'entreprise}

Les résultats obtenus (tableau 8 ) indiquent que $93,8 \times$ des entrepreneurs intérrogés sont responsables des activités quotidiennes de leur entreprise. De plus, la majorité des entrepreneurs de l'echantillon affirment etre responsables des operations $(90,6$ \%), du marketing $(84,4 \times)$, des ressources humaines $(71,9 x)$, de la création et de 1 'innovation $(81,3$ x) ainsi que des finances $(71,9$ x). D'autre part, les entrepreneurs in- 
Tableau 7

Caracteristiques générales des entreprises

de services du Saguenay-Lac-St-Jean

\begin{tabular}{|c|c|c|c|c|}
\hline & $\mathbf{N}$ & $\mathbf{x}$ & $\overline{\mathbf{x}}$ & $\sigma$ \\
\hline AGE DES ENTREPRISES & & & 7,07 & 4,30 \\
\hline 0 a 6 mois & 15 & 46,9 & & \\
\hline 7 a 12 mois & 13 & 40,6 & & \\
\hline Plus de 12 mois & 3 & 9,4 & & \\
\hline DATE D'ENREGISTEMENT/INCORPORATION & & & 8,90 & 6,92 \\
\hline 0 à 6 mois & 13 & 40,6 & & \\
\hline 7 à 12 mois & 11 & 34,4 & & \\
\hline Plus de 12 mois & 7 & 21,9 & & \\
\hline \multicolumn{5}{|l|}{ FORME LEGALE } \\
\hline Proprietaire unique & 22 & 68,8 & & \\
\hline Société avec associes & 4 & 12,5 & & \\
\hline Compagnie par actions & 6 & 18,8 & & \\
\hline \multicolumn{5}{|l|}{ NOMBRE D'ASSOCIES/ACTIONNAIRES } \\
\hline Aucun associélactionnaire & 22 & 68,8 & & \\
\hline Un associé/actionnaire & 3 & 9,4 & & \\
\hline Deux associes/actionnaires & 3 & 9,4 & & \\
\hline Trois associés/actionnaires & 3 & 9,4 & & \\
\hline MEMBRE DE LA FAMILLE DANS L'ENTREPRISE & & & 0,94 & 1,48 \\
\hline Aucune personne & 18 & 56,3 & & \\
\hline Une personne & 8 & 25,0 & & \\
\hline Trois personnes & 4 & 12,5 & & \\
\hline Plus de trois personnes & 2 & 6,2 & & \\
\hline
\end{tabular}


Tableau 8

Caractéristiques 1 iées aux

activités de l'entreprise

\begin{tabular}{|c|c|c|c|c|c|c|}
\hline & & & $\mathbf{N}$ & $x$ & $\overline{\mathrm{x}}$ & $\sigma$ \\
\hline $\begin{array}{l}\text { RESPONSABLE DES ACTIVITES QUOTIDI } \\
\text { Repondant } \\
\text { Autres }\end{array}$ & IENNES & & $\begin{array}{r}30 \\
2\end{array}$ & $\begin{array}{r}93,8 \\
6,3\end{array}$ & & \\
\hline \multirow{2}{*}{\multicolumn{2}{|c|}{$\begin{array}{l}\text { REPONDANT RESPONSABLE DE: } \\
\text { Opérations } \\
\text { Marketing } \\
\text { Ressources humaines } \\
\text { Création et innovation } \\
\text { Finances }\end{array}$}} & & $\begin{array}{l}29 \\
27 \\
23 \\
26 \\
23\end{array}$ & $\begin{array}{l}90,6 \\
84,4 \\
71,9 \\
81,3 \\
71,9\end{array}$ & & \\
\hline & & & Min. & Max. & $\overline{\mathbf{x}}$ & $\sigma$ \\
\hline $\begin{array}{l}\text { NOMBRE D'HEURES DE TRAVA IL/SEMAIN } \\
\text { Entreprise } \\
\text { Tähes d'execution } \\
\text { Taches de gestion }\end{array}$ & & & $\begin{array}{r}14 \\
10 \\
0\end{array}$ & $\begin{array}{r}126 \\
95 \\
55\end{array}$ & $\begin{array}{l}58,6 \\
39,6 \\
15,9\end{array}$ & $\begin{array}{l}24,6 \\
21,1 \\
15,4\end{array}$ \\
\hline Nombre d'eaployes & $\begin{array}{l}\text { Aucun } \\
(x)\end{array}$ & $\begin{array}{c}1 \\
(x)\end{array}$ & $\begin{array}{c}2 \\
(x)\end{array}$ & $\begin{array}{l}3 \text { et }+ \\
(x)\end{array}$ & $\overline{\mathbf{x}}$ & $\sigma$ \\
\hline $\begin{array}{l}\text { NOMBRE D'EMPLOYES } \\
\text { (incluant proprietaire) } \\
\text { Réguliers } \\
\text { Temps partiel } \\
\text { Temps partiel occasionnel } \\
\text { Bénévoles }\end{array}$ & $\begin{array}{l}3,1 \\
68,8 \\
62,5 \\
87,5\end{array}$ & $\begin{array}{r}62,5 \\
9,4 \\
15,6 \\
3,1\end{array}$ & $\begin{array}{r}12,5 \\
15,6 \\
9,4 \\
3,1\end{array}$ & $\begin{array}{r}21,9 \\
6,3 \\
12,5 \\
6,3\end{array}$ & $\begin{array}{l}1,69 \\
0,69 \\
0,91 \\
0,38\end{array}$ & $\begin{array}{l}1,42 \\
1,26 \\
1,57 \\
1,16\end{array}$ \\
\hline $\begin{array}{l}\text { NB. D'EMPLOYES/CATEGORIE D'EMPLOI } \\
\text { Cadres } \\
\text { Personnel de bureau } \\
\text { Personnel de vente } \\
\text { Personnel executant }\end{array}$ & $\begin{array}{l}18,8 \\
75,0 \\
84,4 \\
46,9\end{array}$ & $\begin{array}{r}71,9 \\
18,8 \\
6,3 \\
9,4\end{array}$ & $\begin{array}{r}3,1 \\
3,1 \\
6,3 \\
12,5\end{array}$ & $\frac{3,1}{28,1}$ & $\begin{array}{l}0,90 \\
0,26 \\
0,19 \\
1,78\end{array}$ & $\begin{array}{l}0,60 \\
0,51 \\
0,54 \\
2,29\end{array}$ \\
\hline
\end{tabular}


térrogés accordent en moyenne 58,6 heures de travail par semaine à leur entreprise. De ce nombre d'heures, une moyenne de 39,6 heures sont consacrées à des tăches d'exécution et une moyenne de 15,9 heures à des tăches de gestion. Les résultats nous demontrent également que $62,5 \%$ des entreprises échantillonnées ne comptent qu'un seul employé régulier, généralement le proprietaire-dirigeant lui-meme. Par ailleurs, plus de 60x des répondants n'ont aucun n'employé temps partiel, temps partiel occasionnel ou bénévole. Bref, on obtient une moyenne de 1,69 employés réguliers, de 0,69 employés à temps partiel et de 0,91 employés à temps partiel occasionnel (ces résultats incluent le proprietaire-dirigeant). On observe egalement, que $71,9 \times$ des entreprises étudiées ne comptent qu'un seul employé cadre et que plus de $75 x$ d'entre elles n'ont aucun effectif dans les catégories personnel de bureau et personnel de vente. Par ailleurs, plus de 50\% des répondants bénéficient des services d'au moins un (1) employé exécutant. En résumé, ces entreprises comptent en moyenne 1,78 employés exécutants et 0,90 employés cadres.

\subsection{CARACTERIST IQUES DE L'ENTREPRENEUR}

\subsubsection{Caractéristiques personnelles des entrepreneurs}

Le tableau 9 présente les caractéristiques personnel les des répondants. Les entrepreneurs rencontrés lors de cette etude sont agés en moyenne de 37,8 ans. L'échantillon se compose de 78,1 $\times$ d'hommes et de $21,9 \times$ de femmes et il s'agit majoritairement d'individus maries ou vivant en union libre $(68,7 \%)$. On remarque également qu'une grande proportion des personnes intérrogees $(62,5 x)$ ont un membre de leur famille immédiate (conjoint, père, mere, frère, soeur ou enfants) en affaires qui, souvent, operent dans le meme secteur d'activités $(35,0 \times$ des cas $)$. 
Tableau 9

Caractéristiques personnelles

des entrepreneurs

\begin{tabular}{|c|c|c|c|c|}
\hline & $\mathbf{N}$ & $x$ & $\bar{x}$ & $\sigma$ \\
\hline $\begin{array}{l}\text { AGE } \\
\qquad \begin{array}{l}20 \text { à } 30 \text { ans } \\
31 \text { à } 40 \text { ans } \\
41 \text { à } 50 \text { ans } \\
51 \text { et plus }\end{array}\end{array}$ & $\begin{array}{r}8 \\
14 \\
7 \\
3\end{array}$ & $\begin{array}{r}25,0 \\
43,7 \\
21,8 \\
9,4\end{array}$ & 37,8 & 10,1 \\
\hline $\begin{array}{l}\text { SEXE } \\
\text { Hommes } \\
\text { Femmes }\end{array}$ & $\begin{array}{r}25 \\
7\end{array}$ & $\begin{array}{l}78,1 \\
21,9\end{array}$ & & \\
\hline $\begin{array}{l}\text { ETAT CIVIL } \\
\text { Marie/union l ibre } \\
\text { Célibataire/sépare/divorcé/veuf }\end{array}$ & $\begin{array}{l}22 \\
10\end{array}$ & $\begin{array}{l}68,8 \\
31,3\end{array}$ & & \\
\hline $\begin{array}{l}\text { MEMBRE DE LA FAMILLE EN AFFAIRES } \\
\text { Activités ayant un lien avec répondant }\end{array}$ & $\begin{array}{r}20 \\
7\end{array}$ & $\begin{array}{l}62,5 \\
21,9\end{array}$ & & \\
\hline $\begin{array}{l}\text { NOMBRE D'ANNEES DE SCOLARITE } \\
\text { Moins de } 12 \text { ans } \\
13 \text { a } 15 \text { ans } \\
16 \text { à } 18 \text { ans } \\
19 \text { et } 20 \text { ans }\end{array}$ & $\begin{array}{r}7 \\
6 \\
14 \\
5\end{array}$ & $\begin{array}{l}21,9 \\
18,8 \\
43,7 \\
15,6\end{array}$ & 15,5 & 3,30 \\
\hline $\begin{array}{l}\text { DIPLOME OBTENU } \\
\text { Secondaire général } \\
\text { Secondaire professionnel } \\
\text { Collégial général } \\
\text { Collégial professionnel } \\
\text { Universitaire }\end{array}$ & $\begin{array}{r}5 \\
3 \\
1 \\
5 \\
17\end{array}$ & $\begin{array}{r}15,6 \\
9,4 \\
3,1 \\
15,6 \\
53,1\end{array}$ & & \\
\hline
\end{tabular}


Tableau 9 (suite)

Caractéristiques personnelles

des entrepreneurs

\begin{tabular}{|c|c|c|c|c|}
\hline & $\mathbf{N}$ & $x$ & $\overline{\mathbf{x}}$ & $\sigma$ \\
\hline $\begin{array}{l}\text { ANNEES D'EXPERIENCE DE TRAVAIL } \\
\text { Moins de } 2 \text { ans } \\
2 \text { a } 5 \text { ans } \\
6 \text { à } 10 \text { ans } \\
11 \text { a } 20 \text { ans } \\
\text { Plus de } 20 \text { ans }\end{array}$ & $\begin{array}{l}3 \\
6 \\
9 \\
9 \\
5\end{array}$ & $\begin{array}{r}9,4 \\
18,8 \\
28,2 \\
28,2 \\
15,6\end{array}$ & 12,4 & 9,67 \\
\hline $\begin{array}{l}\text { EMPLOI ANTERIEUR/NB. D'EMPLOYE } \\
\text { Moins de } 10 \text { empl oyés } \\
11 \text { à } 50 \text { empl oyés } \\
51 \text { à } 250 \text { empl oyés } \\
251 \text { a } 500 \text { employés } \\
\text { Plus de } 500 \text { employés }\end{array}$ & $\begin{array}{r}13 \\
14 \\
1 \\
-3\end{array}$ & $\begin{array}{r}40,6 \\
43,8 \\
3,1 \\
12,5\end{array}$ & & \\
\hline EXPERIENCE DE PROPRIETAIRE DIRIGEANT & 7 & 21,9 & & \\
\hline EXPERIENCE DE FAILLITE & 4 & 12,5 & & \\
\hline $\begin{array}{l}\text { ACQUISITION DE CONNAISSANCE/FORMATION } \\
\text { Gestion } \\
\text { Technique } \\
\text { Secteur } \\
\text { Affaires } \\
\text { Démarrage d'entreprise }\end{array}$ & $\begin{array}{r}14 \\
14 \\
14 \\
12 \\
9\end{array}$ & $\begin{array}{l}43,8 \\
43,8 \\
43,8 \\
37,5 \\
28,1\end{array}$ & & \\
\hline $\begin{array}{l}\text { ACQUISITION DE CONNAISSANCE/EXP. TRAVAIL } \\
\text { Gestion } \\
\text { Secteur } \\
\text { Gestion et secteur }\end{array}$ & $\begin{array}{r}3 \\
10 \\
15\end{array}$ & $\begin{array}{r}9,4 \\
31,3 \\
46,9\end{array}$ & & \\
\hline
\end{tabular}


Les caractéristiques de la formation et des experiences de travail des entrepreneurs rencontrés sont également présentés au tableau $9 . \quad$ Le nombre moyen d'années de scolarité de l'echantillon est de 15,5 annees et 53,1\% des entrepreneurs intérrogés détiennent un diplôme universitaire. De plus, le nombre d'annees moyen d'experience de travail est de 12,4 années. Une proportion de $43,8 \%$ de répondants de l'échantillon béneficient de plus de $d i x$ (10) années d'expérience sur le marché du travail et 84,4 \% d'entre eux ont travaille au sein d'une entreprise ayant cinquante (50) employes et moins. D'autre part, 21,9 \% des répondants ont déja éte proprietaires-dirigeants d'une entreprise avant de se lancer en affaires et $12,5 \%$ d'entre eux ont vécu une experience de faillite. Par ailleurs, $43,8 \%$, des entrepreneurs rencontres affirment avoir acquis des connaissances en gestion, des connaissances techniques et des connaissances du secteur d'activites par leur formation académique. Ils ont également acquis des connaissances en affaires dans $37,5 \%$ des cas et en demarrage d'entreprise dans $28,1 \%$ des cas a partir de cette meme formation académique. Dans le même ordre d'idé, 46,9 des répondants ont acquis des connaissances en gestion et des connaissances du secteur d'activités à partir de leur experience de travail, $31,3 \%$ ont acquis des connaissances du secteur d'activites seulement et $9,4 \%$ ont acquis des connaissances en gestion seulement. Ainsi, un total de 78,2 des entrepreneurs rencontrés ont acquis des connaissances du secteurs d'activites par leur travail et un total de 56,3 \% des connaissances en gestion.

\subsubsection{Motivation et facteur déclencheur}

Tel que discute dans le cadre theorique, la motivation de l'entrepreneur est un element déterminant lors de la création d'une entreprise. 
Globalement, les principales raisons ayant motivé les entrepreneurs à fonder leur entreprise (tableau 10) sont: relever un défi (53,1 \%), créer une entreprise à succès $(34,4$ x), créer mon emploi $(31,3 \quad x)$ et le désir d'indépendance $(31,2 \%)$. Ces résultats globaux sont obtenus par un cumul des trois (3) choix. Plus specifiquement, le facteur mentionne le plus fréquemment comme premier choix par les répondants est "créer mon emploi" $(21,9 x)$. En deuxieme choix, le facteur "relever un défi" obtient le meilleur score $(18,8 \%)$ alors que le facteur "creer une entreprise à succés" est choisi par $25,0 x$ des répondants comme troisieme choix.

Tel que mentionné dans la littérature, habituellement la décision de lancer une entreprise survient suite à un évenement particulier vécu par l'entrepreneur. Dans l'ensemble, les résultats obtenus auprés de l'échantillon d'entreprises de services au Saguenay-Lac-St-Jean (tableau 10) demontrent que les principaux facteurs déclencheurs ayant incité l'entrepreneur à lancer son entreprise sont: l'identification d'une opportunité $(40,6 x)$, le congédiement $(31,3 \times)$, la disposition financiére et professionnelle $(25,0 \times)$, l'offre d'un partenaire $(15,6$ x) et l'insatisfaction au travail $(15,6$ \%). Par ailleurs, le congédiement représente le facteur le plus fréquemment mentionné comme premier choix $(21,9 \times)$ par les entrepreneurs rencontrés et l'identification d'une opportunite $(15,6 \times)$ représente le facteur le plus fréquemment mentionné comme deuxième choix.

\subsubsection{Source de l'idée d'affaires}

Les résultats de cette étude, présentés au tableau 11 , démontrent que les entrepreneurs ont identifié leur idé d'affaires principalement à partir de trois sources, soit: de la PME oú je travaillais $(18,8 x)$, d'une innovation $(18,8 x)$ ou de la demande d'un client $(12,5 x)$. 
Tableau 10

Motivation de l'entrepreneur

a fonder son entreprise

\begin{tabular}{|c|c|c|c|c|c|c|c|c|}
\hline & \multicolumn{2}{|c|}{$\mathrm{CHOIX} 1$} & \multicolumn{2}{|c|}{ CHOIX 2} & \multicolumn{2}{|c|}{ CHOIX 3} & \multicolumn{2}{|c|}{ TOTAL } \\
\hline & $\mathbf{N}$ & $x$ & $\mathbf{N}$ & $x$ & $\mathbf{N}$ & $x$ & $\mathbf{N}$ & $x$ \\
\hline $\begin{array}{l}\text { MOTIVATION } \\
\text { Relever un défi } \\
\text { Creer entreprise à succes } \\
\text { Creer mon emploi } \\
\text { Désir d'independance } \\
\text { Etre mon propre patron } \\
\text { Valorisation personnelle } \\
\text { Faire de l'argent } \\
\text { Réaliser un reve } \\
\text { Occuper mes temps libres } \\
\text { Autonomie financiere } \\
\text { Avoir travail intéressant } \\
\text { Autres }\end{array}$ & $\begin{array}{r}6 \\
1 \\
7 \\
4 \\
4 \\
2 \\
2 \\
1 \\
1 \\
-- \\
-\frac{2}{2}\end{array}$ & $\begin{array}{r}18,8 \\
3,1 \\
21,9 \\
12,5 \\
12,5 \\
6,3 \\
6,3 \\
3,1 \\
3,1 \\
---- \\
--- \\
6,3\end{array}$ & $\begin{array}{l}6 \\
2 \\
1 \\
3 \\
3 \\
3 \\
2 \\
1 \\
3 \\
3 \\
3 \\
1\end{array}$ & $\begin{array}{r}18,8 \\
6,3 \\
3,1 \\
9,4 \\
9,4 \\
9,4 \\
6,3 \\
3,1 \\
3,1 \\
9,4 \\
9,4 \\
3,1\end{array}$ & $\begin{array}{l}5 \\
8 \\
2 \\
3 \\
1 \\
2 \\
2 \\
4 \\
-- \\
-- \\
-- \\
--\end{array}$ & $\begin{array}{r}15,6 \\
25,0 \\
6,3 \\
9,4 \\
3,1 \\
6,3 \\
6,3 \\
12,5 \\
-\cdots-- \\
--- \\
---\end{array}$ & $\begin{array}{r}17 \\
11 \\
10 \\
10 \\
8 \\
7 \\
6 \\
6 \\
4 \\
3 \\
3 \\
3\end{array}$ & $\begin{array}{r}53,1 \\
34,4 \\
31,3 \\
31,3 \\
25,0 \\
21,9 \\
18,8 \\
18,8 \\
12,5 \\
9,4 \\
9,4 \\
9,4\end{array}$ \\
\hline $\begin{array}{l}\text { FACTEUR DECLENCHEUR } \\
\text { Identif. / opportunite } \\
\text { Congediement } \\
\text { Dispose financ. \& profes. } \\
\text { Insatisfaction au travail } \\
\text { offre d'un partenaire } \\
\text { Remise en question } \\
\text { Retour au travail } \\
\text { Fin de contrat, projet } \\
\text { offre support finanancier } \\
\text { Mutation (transfert) } \\
\text { Find'etude } \\
\text { Offre d'un client potentiel } \\
\text { Rérogradation } \\
\text { Autres }\end{array}$ & $\begin{array}{r}6 \\
7 \\
2 \\
3 \\
2 \\
3 \\
1 \\
1 \\
-\frac{1}{2} \\
1 \\
-- \\
--\end{array}$ & $\begin{array}{r}18,8 \\
21,9 \\
6,3 \\
9,4 \\
6,3 \\
9,4 \\
3,1 \\
3,1 \\
-6,3 \\
3,1 \\
-0 .- \\
6,3\end{array}$ & $\begin{array}{r}5 \\
2 \\
3 \\
1 \\
1 \\
1 \\
2 \\
1 \\
2 \\
-- \\
-2 \\
-2 \\
-3\end{array}$ & $\begin{array}{r}15,6 \\
6,3 \\
9,4 \\
3,1 \\
3,1 \\
3,1 \\
6,3 \\
3,1 \\
6,3 \\
---- \\
-6,3 \\
-9,- \\
9,4\end{array}$ & $\begin{array}{r}2 \\
2 \\
3 \\
1 \\
2 \\
-- \\
-- \\
1 \\
-1 \\
-1 \\
-- \\
-- \\
1\end{array}$ & \begin{tabular}{r}
6,3 \\
6,3 \\
9,4 \\
3,1 \\
6,3 \\
\hdashline 3,1 \\
3,1 \\
3,1 \\
3,1
\end{tabular} & $\begin{array}{r}13 \\
11 \\
8 \\
5 \\
5 \\
4 \\
3 \\
3 \\
3 \\
2 \\
2 \\
2 \\
-6\end{array}$ & $\begin{array}{r}40,6 \\
31,3 \\
25,0 \\
15,6 \\
15,6 \\
12,5 \\
9,4 \\
9,4 \\
9,4 \\
6,3 \\
6,3 \\
6,3 \\
18,8\end{array}$ \\
\hline
\end{tabular}


Tableau 11

Source de l'idee d'affalres

\begin{tabular}{llr}
\hline & N & $\mathbf{x}$ \\
\hline & & \\
De la PME où je travaillais & 6 & 18,8 \\
Une innovation & 6 & 18,8 \\
Une demande spécifique d'un client & 4 & 12,5 \\
De la grande entreprise où je travaillais & 3 & 9,4 \\
Une recherche active d'opportunités & 2 & 6,3 \\
Un passe-temps lloisirl & 1 & 3,1 \\
Un deuxieme emploi & 1 & 3,1 \\
Association avec inventeur & -- & ---1 \\
Autre & 6 & 18,8 \\
\hline
\end{tabular}

\subsection{RESULTATS CONCERNANT LA PHASE DE PREDEMARRAGE}

\subsubsection{Caractéristiques générales de la phase de prédémarrage}

Le tableau 12 présente les caractéristiques générales de la phase de prédémarrage. Les entrepreneurs interrogés ont consacré en moyenne 4,59 mois a la préparation de leur projet d'entreprise. De plus, dans $62,5 \%$ des cas, cette préparation s'est effectuée sur une période de moins de trois (3) mois. Par ailleurs, 65,6 \% des entrepreneurs rencontres ont formulé un plan d'affaires et 15,6 \% d'entre eux ont suivi un programme de formation sur la préparation d'un plan d'affaires. Enfin, une proportion de $40,6 \%$ de ces entrepreneurs ont bénéficié du support d'un organisme. 
Tableau 12

Caractéristiques générales de la

phase de prédéararage

\begin{tabular}{|c|c|c|c|c|c|}
\hline & $\mathbf{N}$ & $x$ & & $\overline{\mathbf{x}}$ & $\sigma$ \\
\hline $\begin{array}{l}\text { MOIS DE PREPARATION DU PROJET } \\
0 \text { a } 3 \text { mois } \\
4 \text { a } 6 \text { mois } \\
7 \text { a } 12 \text { mois } \\
\text { Plus d'un an }\end{array}$ & $\begin{array}{r}20 \\
6 \\
5 \\
1\end{array}$ & $\begin{array}{r}62,5 \\
18,8 \\
15,6 \\
3,1\end{array}$ & & 4,59 & 6,54 \\
\hline FORMULATION D'UN PLAN D'AFFAIRES & 21 & 65,6 & & & \\
\hline PROGRAMME DE FORMATION/PLAN D'AFFAIRES & 5 & 15,6 & & & \\
\hline SUPPORT D'UN ORGANISME & 13 & 40,6 & & & \\
\hline \multirow[t]{2}{*}{$\begin{array}{l}\text { OBJECTIFS } \\
\text { Présence d'objectifs } \\
\text { Présence d'objectifs écrits } \\
\text { Horizon temporel } \\
\text { Moins d'un an } \\
1 \text { a } 2 \text { ans } \\
3 \text { à } 5 \text { ans } \\
\text { Non précisé }\end{array}$} & $\begin{array}{r}25 \\
13 \\
\\
8 \\
14 \\
3 \\
4\end{array}$ & $\begin{array}{r}78,1 \\
40,6 \\
25,0 \\
43,8 \\
9,4 \\
12,5\end{array}$ & & & \\
\hline & $\mathbf{N}$ & $\overline{\mathbf{x}}$ & $\sigma$ & MD & $m$ \\
\hline $\begin{array}{l}\text { MISSION DE L'ENTREPRISE } \\
\text { Service à offrir } \\
\text { Clientèle visee } \\
\text { Modes d'opération } \\
\text { Territoire vise }\end{array}$ & $\begin{array}{l}32 \\
32 \\
32 \\
32\end{array}$ & $\begin{array}{l}4,47 \\
4,34 \\
4,09 \\
3,59\end{array}$ & $\begin{array}{l}0,84 \\
0,83 \\
1,12 \\
1,21\end{array}$ & $\begin{array}{l}5,00 \\
5,00 \\
4,50 \\
4,00\end{array}$ & $\begin{array}{l}5,00 \\
5,00 \\
5,00 \\
4,00\end{array}$ \\
\hline
\end{tabular}

D'autre part, $78,1 \times$ des entrepreneurs ont affirméavoir fixédes objectifs s'echelonnant, dans la majorité des cas $(68,8 \%)$, sur une période de deux $(2)$ ans et moins. Toutefois, seulement $40,6 \%$ des entrepreneurs interroges ont formulé par ecrit ces memes objectifs. Par ail- 
leurs, il semble que les entrepreneurs interrogés aient assez bien défini la mission de leur future organisation lors de la preparation du projet d'entreprise. En effet, sur une échelle de type likerten cinq points $(1=$ peu et $5=$ beaucoup $)$ les degrés de précision des variables stratég ques motrices sont: service a offrir $(X=4,47)$, territolre vise $(X=3,59)$, clientele visée $(x=4,34)$ et modes d'operation $(x=4,09)$.

\subsubsection{Connaissance du secteur d'activité et de la gestion}

Les résultats présentés au tableau 13 évaluent sur une échelle en cinq points $11=$ pas du tout et $5=$ Tout à fait $)$ le degré de connaissance du secteur d'activités et de la gestion que possédaient les entrepreneurs interrogés avant de prendre la décision finale de se partir en affaires. Ces résultats demontrent que les entrepreneurs avaient de bonnes connaissances du secteur d'activités de leur entreprise et de la gestion avant de se lancer en affaires puisque l'on obtient un score moyen général de 3,74. Par ailleurs, les entrepreneurs possédaient davantage de connaissances concernant les principaux concurrents $(4,13)$, les principaux fournisseurs $(4,13)$ et les clients intéresses $(4,00)$. Toutefois, il semble que le volume potentiel de ventes de la future entreprise était moins connu (score moyen de 2,68 ) des entrepreneurs avant 1 e lancement de la nouvelle entreprise.

\subsubsection{Comportements de gestion des entrepreneurs lies a 1 'analyse des environnements général, sectoriel et interne}

Cette partie présente les résultats détaillés concernant les comportements de gestion des entrepreneurs liés à l'analyse des environnements 


\section{Tableau 13}

Connaissance du secteur d'activité et de la gestion de l'entrepreneur avant de décider de partir en affaires

CONNA ISSANCE DE:

$\begin{array}{llllll}\text { Principaux concurrents } & 32 & 4,13 & 1,03 & 5,00 & 5,00 \\ \text { Principaux fournisseurs } & 32 & 4,13 & 0,18 & 4,00 & 5,00 \\ \text { Clients interesses } & 32 & 4,00 & 0,88 & 4,00 & 4,00 \\ \text { Somme de travail } & 32 & 3,97 & 0,97 & 4,00 & 5,00 \\ \text { Besoin en main-d'oeuvres } & 32 & 3,88 & 1,19 & 4,00 & 5,00 \\ \text { Investissement requis } & 32 & 3,88 & 1,13 & 4,00 & 5,00 \\ \text { Rôle de l'innovation } & 32 & 3,72 & 1,25 & 4,00 & 4,00 \\ \text { Point mort } & 32 & 3,63 & 1,15 & 4,00 & 5,00 \\ \text { Tendance du marché } & 32 & 3,59 & 0,98 & 4,00 & 4,00 \\ \text { Sources de financement } & 32 & 3,56 & 1,59 & 4,00 & 5,00 \\ \text { Volume potentiel de ventes } & 32 & 2,68 & 1,06 & 3,00 & 3,00\end{array}$

géneral, sectoriel et interne. Ces comportements ont été evalués à partir d'une echelle de type Likert en cinq points où "1" (pas du tout) représente la valeur la moins elevee de l'echelle et "5" (tout a fait) la valeur la plus elevée. Afin de permettre certaines comparaisons, les différents facteurs environnementaux ont été divisés en trois (3) groupes principaux soit: l'environnement général, l'environnement sectoriel et l'environnement interne. Par ailleurs l'environnement sectoriel est éga- 
lement subdivisé en quatre (4) sous-groupes, soit: la disponibilité des ressources, les clients, les fournisseurs et les concurrents. L'environnement interne, quant à lui, est compose de six (6) sous-groupes comprenant les aspects marketing, operationnels, humains, financiers, informationnels et légaux.

\subsubsection{Conportenents de gestion des entrepreneurs liés à l'analyse des facteurs de l'environnement général.}

Les résultats descriptifs obtenus (tableau 14) à partir d'une échelle de likert en cinq point $(1=$ pas du tout et $5=$ Tout a fait) nous permettent de constater que les entrepreneurs analysent a des degrés différents chacune des variables de l'environnement général. Globalement, le score moyen de l'environnement général est de 3,10.

Les normes environnementales $(3,63)$, la fiscalite $(3,61)$ et la conjoncture économique $(3,59)$ sont les variables pour lesquelles ont obtient les scores moyens les plus elevés. Par ailleurs, la valeur du dollar canadien $(2,16)$ represente la variable ayant obtenu le score moyen le plus faible. On observe également, que la fiscalité $(3,61)$ représente le facteur le plus analysé parmi les lois et réglements mentionnés alors que la CSST $(2,65)$ correspond au facteur le moins analysé.

\subsubsection{Comportements de gestion des entrepreneurs liés a l'analyse des facteurs de l'environnenent sectoriel}

L'utilisation d'une echelle de Likert en cinq points $11=$ pas du tout et $5=$ tout à fait ' nous a permis d'obtenir les résultats descriptifs pré- 
Tableau 14

Comportenents de gestion de l'entrepreneur liés

a l'analyse des facteurs de l'environnement genéral

lors de la phase de prédémarrage

\begin{tabular}{|c|c|c|c|c|c|}
\hline & $\mathbf{N}$ & $\bar{x}$ & $\sigma$ & $M D$ & MO \\
\hline Normes environnementales & 32 & 3,63 & 1,59 & 4,00 & 5,00 \\
\hline Lois/Fiscalité & 32 & 3,61 & 1,20 & 4,00 & 4,00 \\
\hline Conjoncture économique & 32 & 3,59 & 1,24 & 4,00 & 4,00 \\
\hline Temperature et saison & 32 & 3,56 & 1,34 & 4,00 & 4,00 \\
\hline Taux de chômage & 32 & 3,48 & 1,47 & 4,00 & 4,00 \\
\hline Lois/Santé et hygiène & 32 & 3,38 & 1,43 & 4,00 & 5,00 \\
\hline Lois/Normes du travail & 32 & 3,38 & 1,38 & 3,00 & 3,00 \\
\hline Technologies disponibles & 32 & 3,31 & 1,54 & 4,00 & 4,00 \\
\hline Subventions gouvernem. & 32 & 3,15 & 1,70 & 4,00 & 5,00 \\
\hline Taux d'interet & 32 & 3,10 & 1,55 & 3,00 & 1,00 \\
\hline Lois/Batiment & 32 & 3,04 & 1,52 & 4,00 & 4,00 \\
\hline Aides gouvernementales & 32 & 2,81 & 1,58 & 3,00 & 1,00 \\
\hline Lois/Municipal & 32 & 2,81 & 1,27 & 3,00 & 4,00 \\
\hline Taux d'inflation & 32 & 2,67 & 1,49 & 2,00 & 1,00 \\
\hline Lois/CSST & 32 & 2,65 & 1,38 & 2,00 & 2,00 \\
\hline Tendances démographiques & 32 & 2,33 & 1,43 & 2,00 & 1,00 \\
\hline Valeur du dollar canadien & 32 & 2,16 & 1,39 & 1,00 & 1,00 \\
\hline SCORE MOYEN GLOBAL & & 3,10 & & & \\
\hline
\end{tabular}

sentés au tableau 15. Le score moyen global pour l'envi ronnement sectoriel est de 3,49. De plus, I'observation des scores moyens nous permet de constater que les variables de l'environnement sectoriel sont analysés à des degrés différents. Les besoins de la clientele $(4,38)$, la 
Tableau 15

Conportenent de gestion de l'entrepreneur liés

A l'analyse des facteurs de l'environnement sectoriel

lors de la phase de prédémarrage

\begin{tabular}{|c|c|c|c|c|c|}
\hline & $\mathbf{N}$ & $\overrightarrow{\mathbf{x}}$ & $\sigma$ & $M D$ & MO \\
\hline RESSOURCES (score moyen global): & & 3,81 & & & \\
\hline Disponibili té/équipements & 32 & 4,29 & 0,90 & 5,00 & 5,00 \\
\hline Disponib./prod.\& fournitures & 32 & 4,10 & 1,15 & 4,50 & 5,00 \\
\hline Disponibilite/ress.humaines & 32 & 3,03 & 1,48 & 3,00 & 4,00 \\
\hline LES CLIENTS (score moyen global) & & 2,97 & & & \\
\hline Besoins de la clientele & 32 & 4,38 & 0,83 & 4,50 & 5,00 \\
\hline Identif./types de clienteles & 32 & 3,97 & 1,09 & 4,00 & 4,00 \\
\hline Taille du marché & 32 & 3,63 & 1,24 & 4,00 & 4,00 \\
\hline Caractér./territoire visé & 32 & 3,19 & 1,25 & 3,00 & 4,00 \\
\hline Ouverture marchélautres prov. & 32 & 1,67 & 1,28 & 1,00 & 1,00 \\
\hline Poss.exportation hors Canada & 32 & 1,00 & 0,00 & 1,00 & 1,00 \\
\hline \multicolumn{6}{|c|}{ LES FOURNISSEURS (score moyen global): 3,63} \\
\hline Qualité des services & 32 & 3,84 & 1,19 & 4,00 & 5,00 \\
\hline Localisation & 32 & 3,81 & 1,20 & 4,00 & 5,00 \\
\hline Caractér./produits-services & 32 & 3,81 & 1,20 & 4,00 & 4,00 \\
\hline Prix des prod.et services & 32 & 3,74 & 1,13 & 4,00 & 4,00 \\
\hline Nombre & 32 & 3,63 & 1,22 & 4,00 & 4,00 \\
\hline Délais de livraison & 32 & 3,63 & 1,16 & 4,00 & 4,00 \\
\hline Quantités disponibles & 32 & 3,52 & 1,43 & 4,00 & 4,00 \\
\hline Volontéfaire aff. ensemble & 32 & 3,45 & 1,34 & 4,00 & 4,00 \\
\hline Termes de crédits & 32 & 3,25 & 1,48 & 3,50 & 5,00 \\
\hline
\end{tabular}


Tableau 15 (suite)

Comportement de gestion de l'entrepreneur lies

à l'analyse des facteurs de l'environnement sectoriel

lors de la phase de predemarrage

\begin{tabular}{|c|c|c|c|c|c|}
\hline & $N$ & $\overline{\mathbf{X}}$ & $\sigma$ & MD & MO \\
\hline LES CONCURRENTS I score moyen & ball: & 3,55 & & & \\
\hline Localisation & 32 & 4,00 & 1,22 & 4,00 & 5,00 \\
\hline Qualité des services & 32 & 4,00 & 1,00 & 4,00 & 4,00 \\
\hline Prix des services & 32 & 3,94 & 1,03 & 4,00 & 5,00 \\
\hline Nombre & 32 & 3,91 & 1,40 & 4,00 & 5,00 \\
\hline Caracter. des services & 32 & 3,80 & 1,16 & 4,00 & 4,00 \\
\hline Part de marché & 32 & 3,59 & 1,34 & 4,00 & 5,00 \\
\hline Forces-faiblaisses/concurr. & 32 & 3,58 & 1,43 & 4,00 & 5,00 \\
\hline Distribution & 32 & 3,44 & 1,16 & 3,00 & 3,00 \\
\hline Publicité et promotion & 32 & 3,39 & 1,26 & 3,00 & 3,00 \\
\hline Arrivée nouveaux concurr. & 32 & 2,74 & 1,37 & 2,00 & 2,00 \\
\hline Services substituts & 32 & 2,63 & 1,28 & 3,00 & 3,00 \\
\hline SCORE MOYEN GLOBAL & & 3,49 & & & \\
\hline
\end{tabular}

disponibilite des equipements $(4,29)$ et la disponibilité des produits et fournitures $(4,10)$ représentent les variables ayant obtenu les scores moyens les plus elevés si l'on considere toutes les variables de l'environnement sectoriel. A l'opposé, les possibilités d'exportation $(1,00)$ et l'ouverture de marché autres provinces $(1,67)$ abtiennent les scores moyens les moins élevés.

Par ailleurs, une analyse détaillée des résultats nous révele d'autres informations intéressantes. Ainsi, nous obtenons un score moyen 
global de 3,81 pour l'analyse de la disponibilite des ressources. Le facteur le plus analyse est la disponibilite des equipements $(4,29)$ tandis que la disponibilité des ressources humaines (3,03) correspond au facteur le moins analysé.

Concernant l'analyse de la clientele, un score moyen de 2,97 a ete obtenu. Les facteurs les plus analysés sont les besoins de la clientele (4,38) et l'identification des types de clienteles (3,97). A l'oppose, les facteurs les moins analysés sont les possibilites d'exportation hors Canada $(1,00)$ et l'ouverture de marché autres provinces $(1,67)$. Soulignons cependant que notre echantillon est constitue majoritairement d'entreprises visant un marché local $(34,4 \%)$ ou regional $(40,6 x)$ (voir tableau 22).

Le score moyen obtenu pour l'analyse des fournisseurs est de 3,63 . Les facteurs les plus analysés sont la qualité des services $(3,84)$, la localisation $(3,81)$, les caractéristiques des produits et services $(3,81)$ ainsi que les prix des produits et services $(3,74)$. Quant aux autres facteurs analysés par les entrepreneurs, ils cumulent des scores moyens qui se situent près de la moyenne globale $(3,63)$.

Finalement, le score moyen lié à l'analyse de la concurrence est de 3,55. La localisation des concurrents $(4,00)$, la qualité des services $(4,00)$, le prix de ces services $(3,94)$ et le nombre de concurrents $(3,91)$ sont les facteurs les plus analysés par les futurs entrepreneurs. Par ailleurs, les facteurs les moins analysés sont les services substitut $(2,63)$ et les possibilités d'arrivée des nouveaux concurrents $(2,74)$. Ces derniers résultats nous apparaissent surprenant compte tenu du fait que plusieurs des entrepreneurs interrogés considerent 1'arrivée de nou- 
veaux concurrents $(28,8 x)$ et les services substitute $(1 \overline{8}, 8 x)$ comme des menaces pour leur entreprise (tableau 16 ).

En résumé pour chacun des sous-groupes de l'environnement sectoriel, nous observons les scores moyens suivants (en ordre décroissant): disponibilité des ressources $(3,81)$, fournisseurs $(3,63)$, concurrents $(3,55)$ et clients $(2,97)$. Nous devons cependant etre prudent concernant la dimension clients puisque deux facteurs louverture des marchés autres provinces et possibilités d'exportation hors Canadal viennent abaisser considérablement ce résultat. En effet, nous obtenons un score moyen de 3,79 pour cette dimension, si l'on ne considere pas ces deux facteurs dans le calcul de la moyenne. Finalement, nous obtenons un scoremoyen globale de 3,49 pour l'environnement sectoriel.

\subsubsection{Dpportunité et menaces de l'environnement externe}

L'analyse des environnements genéral et sectoriel permet à l'entrepreneur d'identifier les facteurs externes qui peuvent representer des opportunités ou des menaces pour la future entreprise. Les résultats de cette etude (tableau 16) démontrent que 37,5 x des entrepreneurs interrogés considérent les besoins de la clientele come un facteur favorisant la création et le demarrage de leur entreprise alors que $21,9 \times$ des répondants mentionnent au meme titre, le nombre de concurrents et 18,8x, la conjoncture économique. En contre partie, 53,1X des entrepreneurs considerent la conjoncture économique comme l'un des principaux facteurs pouvant menacer la création et le démarrage de leur entreprise. De plus, certains entrepreneurs affirment que l'arrivee de nouveaux concurrents $(28,2 x)$ et les services substituts $(18,8$ \%) constituent des menaces importantes lors du lancement de leur entreprise. 
Tableau 16

Facteurs considérés conne une

opportunlté ou une menace par l'entrepreneur

lors de la phase de prédémarrage

\begin{tabular}{|c|c|c|c|c|c|c|c|c|}
\hline & \multicolumn{2}{|c|}{ CHDIX 1} & \multicolumn{2}{|c|}{$\mathrm{CHOIX} 2$} & \multicolumn{2}{|c|}{ CHOIX 3} & \multicolumn{2}{|c|}{ TOTAL } \\
\hline & $\mathbf{N}$ & $x$ & $\mathbf{N}$ & $x$ & $\mathbf{N}$ & $x$ & $\mathbf{N}$ & $x$ \\
\hline $\begin{array}{l}\text { OPPORTUNITES } \\
\text { Besoins clientele } \\
\text { Nombre de concurrents } \\
\text { Conjoncture economique } \\
\text { Taille du marché } \\
\text { Subventions gouvernement. } \\
\text { Prix des serv. concurr. } \\
\text { Normes environnement. } \\
\text { Local isation/concurr. } \\
\text { Taux d'interet } \\
\text { ldentif.types de client. } \\
\text { Prix prod.serv./fourn. } \\
\text { Part de marche/concurr. } \\
\text { Temperature et saison } \\
\text { Technologie disponible } \\
\text { Disponib. ress. humaines } \\
\text { Aides gouvernementales } \\
\text { Localisation/fournis. }\end{array}$ & $\begin{array}{r}9 \\
3 \\
4 \\
2 \\
1 \\
1 \\
-1 \\
1 \\
2 \\
1 \\
1 \\
1 \\
1 \\
1 \\
-- \\
-- \\
--\end{array}$ & $\begin{array}{r}28,1 \\
9,4 \\
12,5 \\
6,3 \\
3,1 \\
3,1 \\
3,1 \\
6,3 \\
3,1 \\
3,1 \\
3,1 \\
3,1 \\
3,1 \\
- \\
-\end{array}$ & $\begin{array}{r}1 \\
3 \\
1 \\
2 \\
2 \\
2 \\
3 \\
1 \\
-1 \\
1 \\
1 \\
1 \\
-1 \\
-1 \\
1 \\
1 \\
1\end{array}$ & $\begin{array}{r}3,1 \\
9,4 \\
3,1 \\
6,3 \\
6,3 \\
6,3 \\
9,4 \\
3,1 \\
-3,1 \\
3,1 \\
3,1 \\
-0 \\
3,1 \\
3,1 \\
3,1\end{array}$ & $\begin{array}{l}2 \\
1 \\
1 \\
1 \\
2 \\
1 \\
1 \\
1 \\
-- \\
-- \\
-- \\
-- \\
1 \\
1 \\
1 \\
1 \\
1\end{array}$ & \begin{tabular}{r}
6,3 \\
3,1 \\
3,1 \\
3,1 \\
6,3 \\
3,1 \\
3,1 \\
3,1 \\
\hdashline-1 \\
\hdashline-1 \\
3,1 \\
3,1 \\
3,1 \\
3,1 \\
3,1
\end{tabular} & $\begin{array}{r}12 \\
7 \\
6 \\
5 \\
5 \\
4 \\
4 \\
3 \\
2 \\
2 \\
2 \\
2 \\
2 \\
2 \\
2 \\
2 \\
2\end{array}$ & $\begin{array}{r}37,5 \\
21,9 \\
18,8 \\
15,6 \\
15,6 \\
12,5 \\
12,5 \\
9,4 \\
6,3 \\
6,3 \\
6,3 \\
6,3 \\
6,3 \\
6,3 \\
6,3 \\
6,3 \\
6,3\end{array}$ \\
\hline $\begin{array}{l}\text { MENACES } \\
\text { Conjoncture économique } \\
\text { Arrivee nouv. concurr. } \\
\text { Services substituts } \\
\text { Part de marchelconcur. } \\
\text { Taux d'inflation } \\
\text { Prix des serv. concur. } \\
\text { Qualite/serv. concurrent } \\
\text { Taille du marche } \\
\text { Nombre de concurrents } \\
\text { Caracter./serv. concur. } \\
\text { Subventions gouvern. } \\
\text { Publicite et promotion }\end{array}$ & $\begin{array}{r}15 \\
3 \\
2 \\
2 \\
1 \\
1 \\
1 \\
-- \\
-- \\
-- \\
--\end{array}$ & $\begin{array}{r}46,9 \\
9,4 \\
6,3 \\
6,3 \\
3,1 \\
3,1 \\
3,1 \\
---- \\
--- \\
---\end{array}$ & $\begin{array}{r}1 \\
-\frac{4}{1} \\
1 \\
-1 \\
-2 \\
2 \\
2 \\
1 \\
1\end{array}$ & \begin{tabular}{r}
3,1 \\
12,5 \\
\hdashline 3,1 \\
3,1 \\
3,1 \\
$-6,3$ \\
6,3 \\
6,3 \\
3,1 \\
3,1
\end{tabular} & $\begin{array}{r}1 \\
2 \\
4 \\
-- \\
-- \\
-1 \\
-- \\
-- \\
-1 \\
1\end{array}$ & 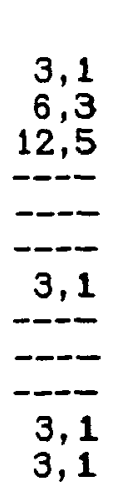 & $\begin{array}{r}17 \\
9 \\
6 \\
3 \\
2 \\
2 \\
2 \\
2 \\
2 \\
2 \\
2 \\
2\end{array}$ & $\begin{array}{r}53,1 \\
28,2 \\
18,8 \\
9,4 \\
6,3 \\
6,3 \\
6,3 \\
6,3 \\
6,3 \\
6,3 \\
6,3 \\
6,3\end{array}$ \\
\hline
\end{tabular}


4.4.3.4 Comportements de gestion des entrepreneurs lís à l'analyse des facteurs de l'environnement interne

\begin{abstract}
L'utilisation d'une echelle de Likert en cinq points ( $1=$ pas du tout et $5=$ tout à fait) nous a permis d'obtenir les resultats descriptifs concernant les comportements de gestion liés à l'analyse des facteurs de l'environnement interne (tableau 17). Ainsi, le score moyen global de l'environnement interne est de 3,45. Par ailleurs, les differents facteurs liés à l'environnement interne ont été regroupés en six (6) aspects soit les aspects marketing, opérationnels, humains, financiers, informationnels et légaux. L'observation des scores moyens obtenus, nous permet de constater que les entrepreneurs interrogés analysent à des degrés différents chacun des facteurs de l'environnement interne.
\end{abstract}

Nous obtenons un score moyen de 3,75 pour l'analyse des aspects marketing. La qualité des services à offrir (4,50), le service à la clientele $(4,10)$ et la gamme de services à offrir $(4,09)$ sont les facteurs marketing les plus analyses tandis que les sources d'information (3,13) correspondent au facteur le moins analysé par les entrepreneurs rencontrés dans le cadre de cette étude. De plus, on observe que le prix du service à offrir $(3,84)$, l'uniformisation du service offert (3,79), les délais de livraison $(3,77)$ et l'accessibilité aux services $(3,68)$ enregistrent des scores prets de la moyenne globale des aspects marketing. Soulignons également, que la qualitédes services à offrir $(4,50)$ représente le facteur ayant obtenu le score moyen le plus élevé de l'ensemble des facteurs des environnements général, sectoriel et interne.

Concernant les aspects operationnels, nous obtenons un score moyen de 3,51 et l'analyse des besoins en equipement et fournitures $(4,13)$ est le facteur le plus analysé. Cette observation, nous semble intéressante 
Tableau 17

Comportements de gestion de l'entrepreneur

llés à l'analyse de l'environnement interne

lors de la phase de prédémarrage

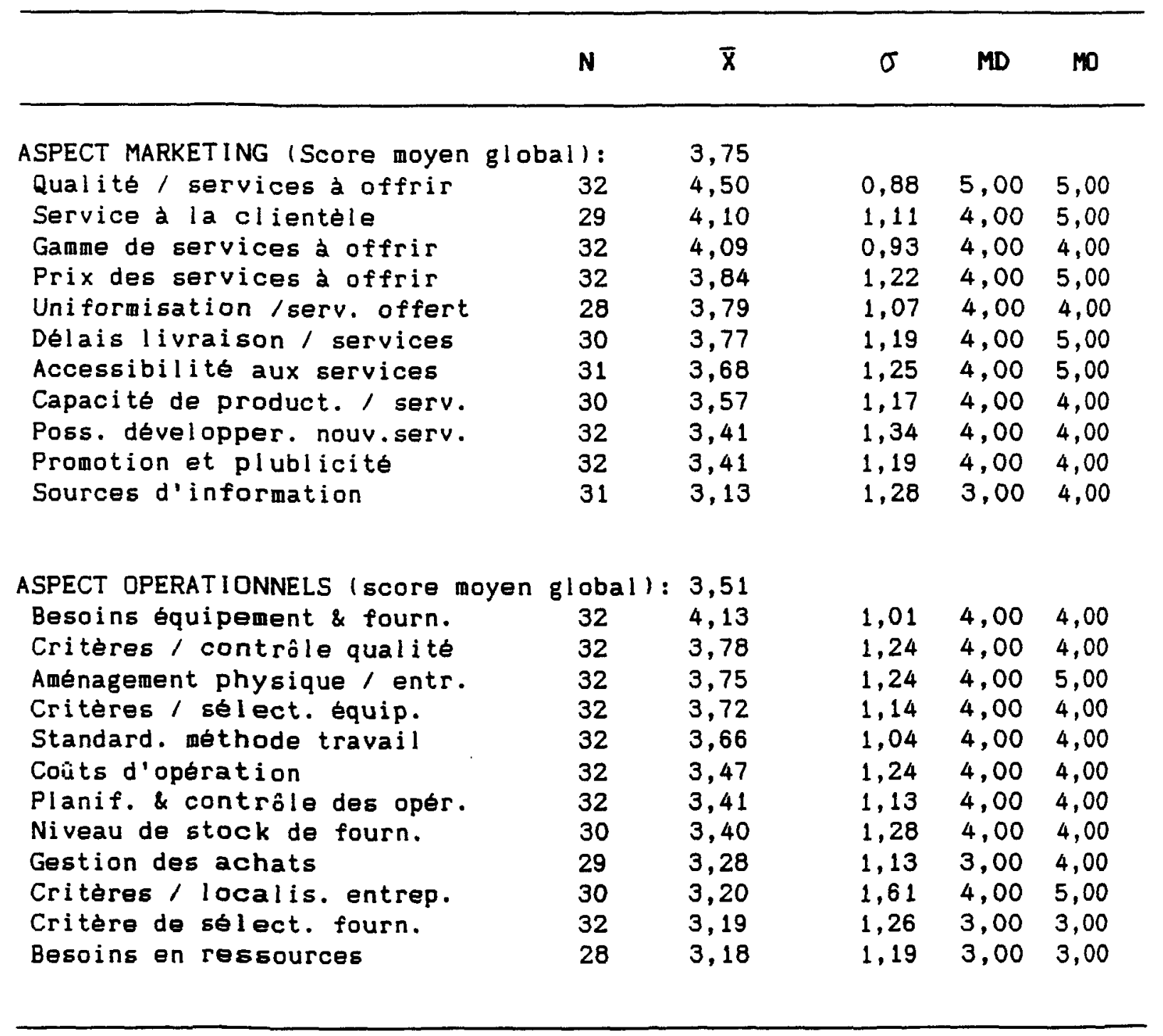


Tableau 17 (suite)

Comportements de gestion de l'entrepreneur

liés à l'analyse de l'environnement interne

lors de la phase de predemarrage

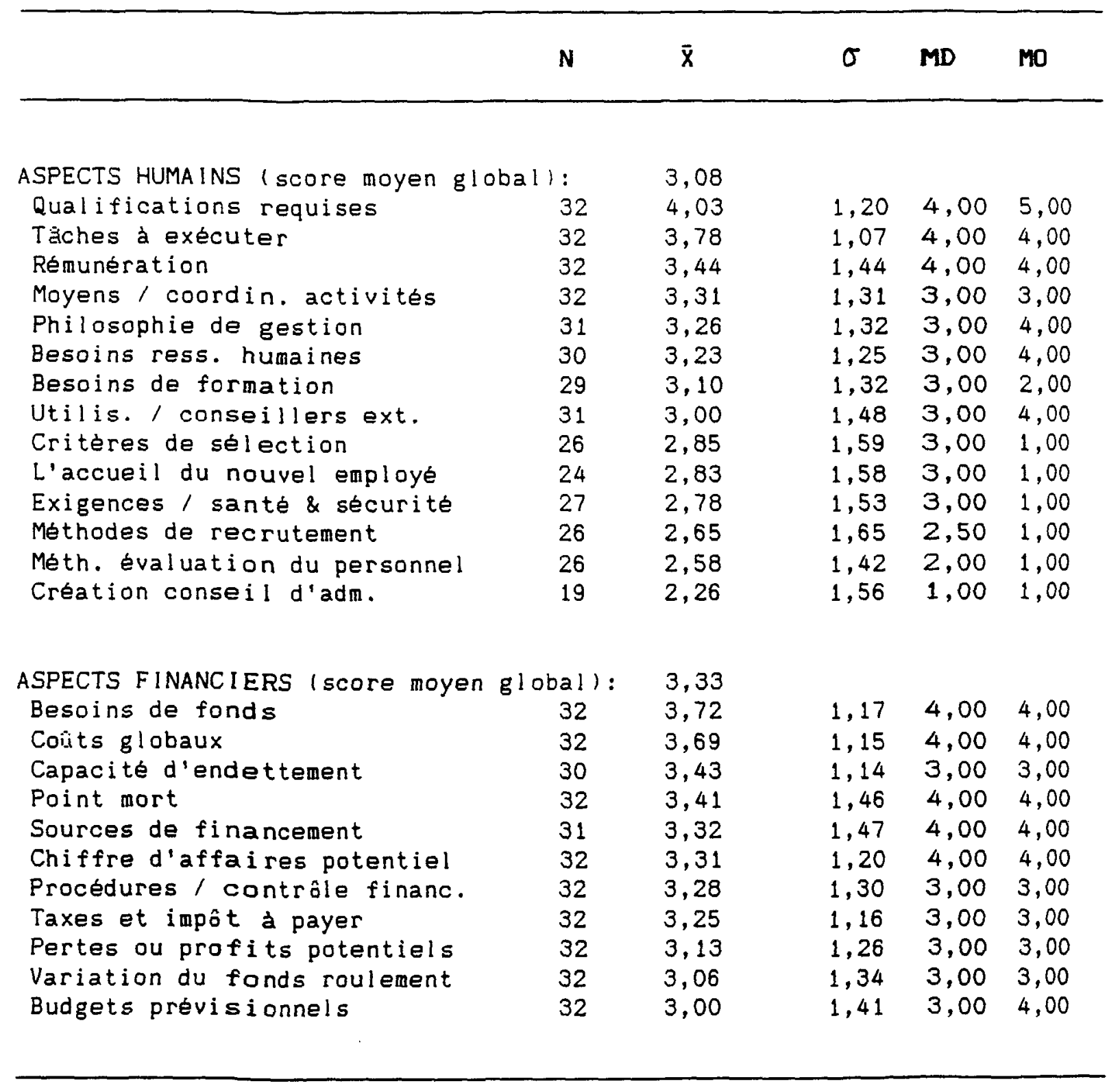




\section{Tableau 17 (suite)}

\section{Conportements de gestion de l'entrepreneur}

llés a l'analyse de l'environnement interne

lors de la phase de prédémarrage

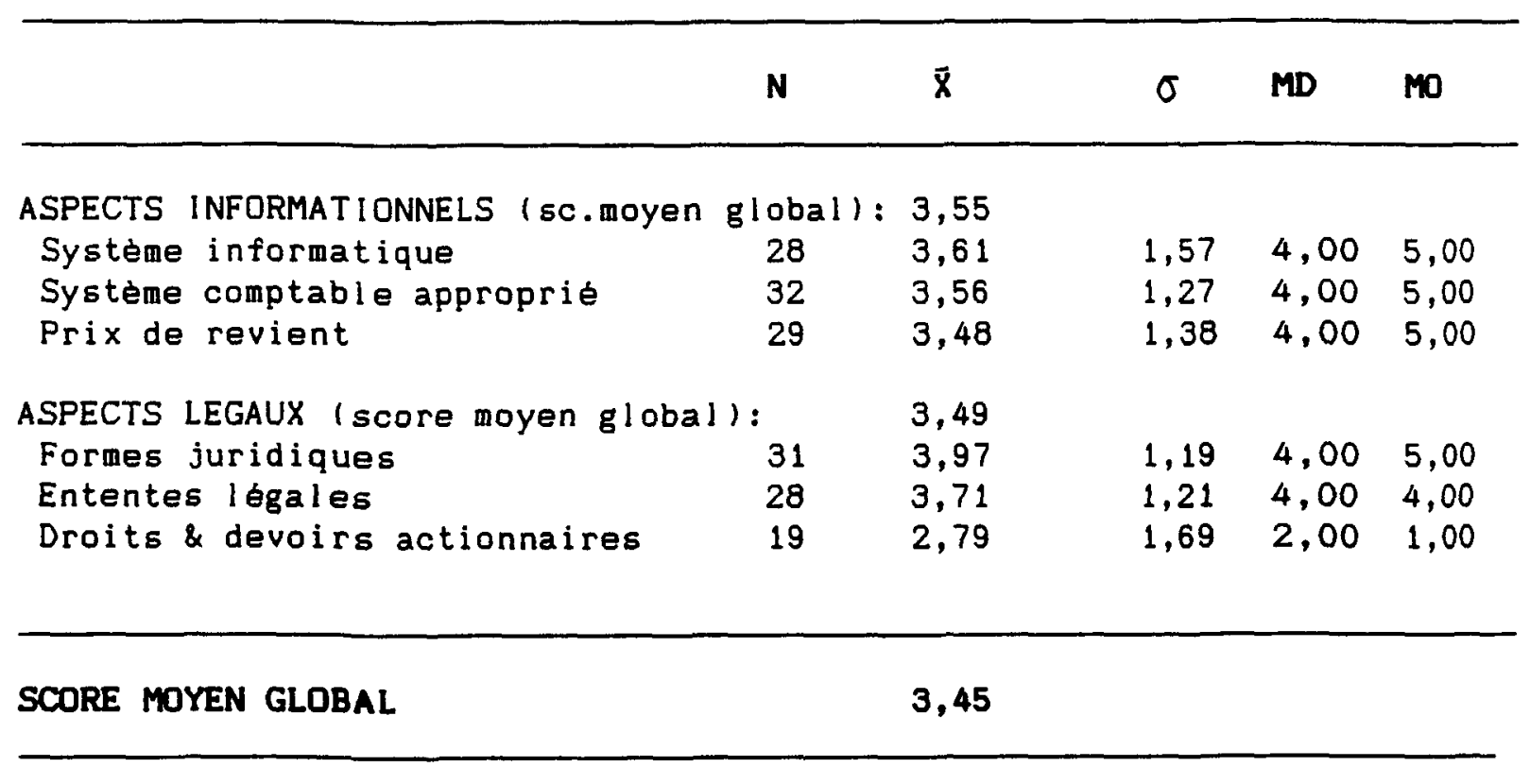

considérant que lors de l'analyse de l'environnement sectoriel, nous avons obtenu des scores elevés concernant les facteurs "disponibilité en équipement" $(4,29)$ et "disponibilité des produits et fournitures" $(4,10)$ (voir tableau 15). Par ailleurs, les criteres de contrôle de la qualite $(3,78)$, l'aménagement physique de l'entreprise $(3,75)$, les critéres de sélection des equipements $(3,72)$ et la standardisation des méthodes de travail $(3,66)$ obtiennent des scores moyens supérieurs a la moyenne global de l'aspect opérationnel. Quant aux besoins en ressources (3,18), aux criteres de sélection des fournitures $(3,19)$ et aux critères de localisation de l'entreprise $(3,20)$, ils correspondent aux facteurs opérationnels les moins analysés. 
Les aspects humains obtiennent la moyenne la plus falble de l'environnement interne avec un score de 3,08 . Ce résultat, nous semble lié au fait que les entreprises echantillonnees comptent en moyenne 1,69 employés réguliers et ce, en incluant le proprietaire-dirigeant. D'autre part, les facteurs les plus analysés par les entrepreneurs sont les qualifications requises $(4,03)$ et les tăches à exécuter $(3,78)$. En outre, les critères de selection (3,85), l'accueil du nouvel emplayé $(2,83)$, les exigences en santé et sécurité $(2,78)$, les méthodes de recrutement $(2,65)$ et les méthodes d'évaluation du personnel $(2,58)$ enregistrent des scores inférieurs à la moyenne globale de l'aspect humain. La création d'un conseil d'administration $(2,26)$ represente, quant à lui, le facteur humain le moins analysé par l'entrepreneur, lors de la phase de prédemarrage.

Pour les aspects financiers, nous obtenons un score moyen de 3,33 . Les facteurs les plus analyses sont les besoins de fonds $(3,72)$ et les coúts globaux $(3,69)$ alors que le facteur le moins analysé correspond aux budgets prévisionnels $(3,00)$. Quant à la capacitéd'endettement $(3,43)$, l'analyse du point mort $(3,41)$, les sources de financement $(3,32)$, le chiffre d'affaires potentiel $(3,31)$, les procédures de contrôle financier $(3,28)$ et les taxes et impôt à payer $(3,25)$, ils obtiennent des scores prêts de la moyenne globale de l'aspect financier.

Concernant les aspects informationnels, nous observons que les scores moyens obtenus pour les differents facteurs analysés se situent autour du score moyen global $(3,55)$. De façon plus précise, le systeme informatique, le systeme comptable et le prix de revient enregistrent respectivement des scores de $3,61,3,56$ et 3,48 . 
Nous cumulons un score moyen de 3,49 pour les aspects légaux. Les formes furidiques $(3,97)$ representent le facteur le plus analyse tandis que les droits et devoirs des actionnaires $(2,79)$ constituent le facteur le molns analysé. Cependant, ce dernier résultat peut s'expliquer par le fait que $68,8 \times$ des répondants possèdent une entreprise à propriétaire unique.

En résumé, par ordre décroissant, nous obtenons les scores moyens suivants: aspects marketing $(3,75)$, aspects informationnels $(3,55)$, aspects operationnels $(3,51)$, aspects légaux $(3,49)$, aspects financiers $(3,33)$ et aspects humains $(3,08)$. Ce qui nous donne un score moyen globale de 3,45 concernant l'analyse de l'environnement interne par les entrepreneurs lors de la phase de predémarrage.

\subsubsection{Forces et faiblesses de l'entreprise}

Les résultats du tableau 18 indiquent que les répondants considerent leurs caractéristiques personnelles $(56,3 \%)$, les caractéristiques du service offert par leur entreprise $(31,3 \%)$ et la connaissance du secteur d'activités $(31,3 \%)$ comme les principales forces pouvant avantager la future entreprise. A l'opposé, les principales faiblesses identifiées par les entrepreneurs sont leurs connaissances de la gestion $(21,9 \times)$ et les finances de l'entreprise $(18,6$ \%). De plus, l'analyse des résultats obtenus auprés de cet echantillon permet également de constater que les entrepreneurs ont identifie davantage de forces que de faibl esses. 
Tableau 18

\author{
Forces et Faiblesses des entreprises de \\ services en phase de predemarrage
}

\begin{tabular}{llc}
\hline & N & \\
& & \\
& & \\
& & \\
FORCES & 18 & 56,3 \\
Personnalité des dirigeants & 10 & 31,3 \\
Caracteristiques du service & 10 & 31,3 \\
Connaissance du secteur d'activités & 8 & 25,0 \\
Service innovateur & 7 & 21,9 \\
Qualité du service & 6 & 18,6 \\
Financement de l'entreprise & 6 & 18,6 \\
Localisation de l'entreprise & 3 & 9,4 \\
Caractéristiques de l'entreprise & \\
\hline FAlBlesses & & \\
& & \\
Connaissance de la gestion & 7 & 21,9 \\
Finance de l'entreprise & 6 & 18,6 \\
Promotion et publ icité & 4 & 12,5 \\
Localisation de l'entreprise & 2 & 6,3 \\
Personnalité du dirigeant & 2 & 6,3 \\
& & \\
\hline
\end{tabular}

\title{
4.4.5 Sources d'information
}

Lors de l'analyse des environnements interne et externe, diverses sources d'informations (impersonnelles et personnelles) peuvent etre consultées. Nous avons tenté d'évaluer la fréquence d'utilisation ainsi que le degré d'intéret, sur une echelle de likert en cinq points ( $1=$ pas intéressant et $5=$ tres intéressant), des principales sources d'informations 
consultees par les entrepreneurs lors de la preparationde leur projet d'entreprise. Les résultats présentés au tableau 19 démontrent que les entrepreneurs rencontres considerent que la plupart des sources d'information consultees sont intéressantes. De plus, les futurs proprietairesdirigeants consultent les revues et journaux $(10 / 32-31,2 x)$, les amis $(9 / 32-28,1 x)$, les clients $(8 / 32=25,0 x)$ et les parents $(6 / 32-18,8 x)$ pour obtenir de l'information sur l'economie. Toutefois, les amis (source personnelle) obtiennent le score moyen le plus eleve $(4,11)$ concernant le degré d'intéret des informations sur l'economie recueillies par les entrepreneurs. D'autre part, 21,9x (7/32) des entrepreneurs rencontrés consultent les revues et journaux et $18,6 \times(6 / 32)$ les amis, sur les questions d'ordre politique. Ces deux sources d'information politique (revue \& journaux et amis) obtiennent des scores moyens d'environ 3,50 .

Les bibliotheques et les ministères $(6 / 32-18,8 \%)$ sont les principales sources d'information concernant l'aide gouvernementale disponible. Cependant, l'interet démontré par les entrepreneurs pour ces sources d'information se traduit par un score moyen de 2,67 seulement. Les bibliotheques et ministères $(8 / 32-25,0 x)$ constituent également une source d'information privilégiée par les entrepreneur pour obtenir de l'information sur les lois et réglements. On obtient alors un score moyen de 3,88 concernant le degré d'interet des informations sur les lois et réglements obtenues de cette source (bibliotheques et ministéres). Par ailleurs, les sources personnelles sont généralement privilégiées par les entrepreneurs afin de recueillir de l'information sur la clientele. Ainsi, les clients $(13 / 32-40,6 x)$, les concurrents $(7 / 32-21,9 x)$ et les amis $(6 / 32-$ $18,8 \%$ sont les principales sources consultées par les entrepreneurs pourobtenir des renseignements sur la clientele. De plus, les entrepreneurs considèrent que les concurrents $(4,43)$ et les clients (4,31) representent les sources d'information les plus intéressantes sur la 


\section{TABLEAU 19}

Les sources d'information consultées et l'intérêt des entrepreneurs concernant les informations obtenues

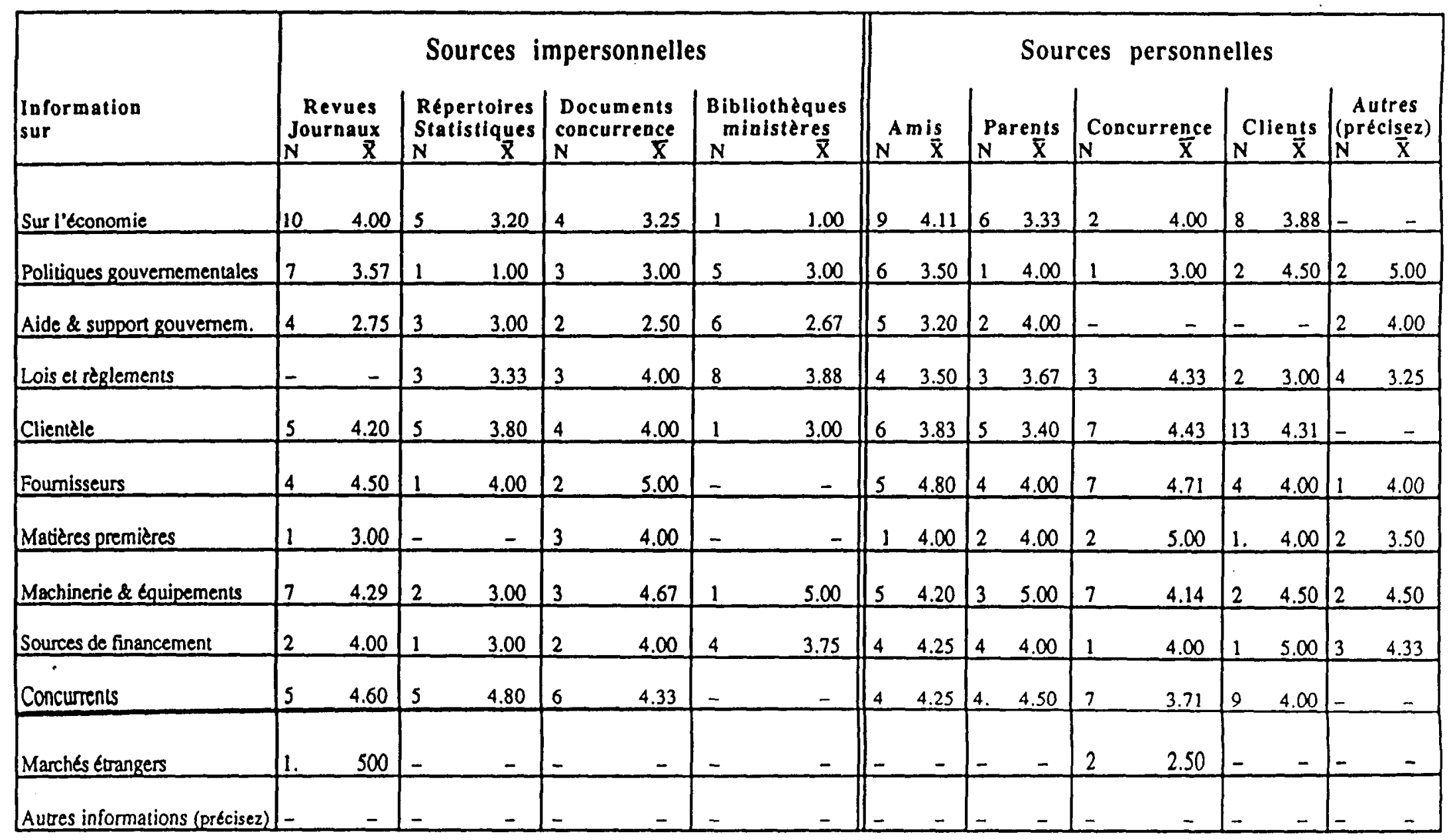


clientele. Concernant les fournisseurs, les repondants s'informent principalement aupres de leurs concurrents $(21,9 x)$ et trouvent particulièrement intéressants les renseignements ainsi recueill is puisque le degré d'intéret de cette source se traduit par un score moyen de 4,71.

Les entrepreneurs consultent géneralement les revues et journaux $(7 / 32-21,9 \%)$ ainsi que les concurrents $(7 / 32-21,9 \%)$ pour se renseigner sur la machinerie et l'équipement et considerent ces sources intéressantes puisqu'ils accordent un score moyen superieur à 4,00 à chacune d'elles. En quete d'informations sur leurs concurrents, les entrepreneurs rencontrés consultent principalement les clients $(9 / 32-28,1 \times)$, les concurrents $(21,9 \%)$ ainsi que la documentation de ces concurrents (6/3218,8\%). Cependant, la documentation des concurrents est considérée comme la source la plus intéressante par les entrepreneurs puisqu'ils lui accordent un score moyen de 4,33 .

Bref, les entrepreneurs rencontrés consultent principalement leurs amis (49 fois), les revues \& journaux (47 fois) et leurs clients 142 foisl pour obtenir les informations necessaires a la preparation de leur projet d'entreprise. Ces consultations ont principalement pour objectifs de recueillir de l'information sur la clientele (46 fois), l'economie (45 fois) et les concurrents ( 40 fois).

\subsubsection{Personnes ressources}

Comme nous l'avons précisé dans le cadre théorique, la création d'une entreprise représente une démarche relativement complexe. La réalisation d'un tel projet implique généralement la participation de plusieurs personnes. Afin d'évaluer le degré d'implication des prin- 
cipales personnes ressources identifiees, nous avons utilisé une échelle de Likert en cinq points $11=$ inactif et $5=$ trés actifl. Les résultats présentés au tableau 20 nous démontrent que les personnes ressources sont généralement peu actives, lors de l'élaboration du projet d'entreprise. Toutefois, la famille $(3,41)$ et les amis $(3,15)$ représentent les personnes ressources les plus actives lors de la phase de prédémarrage. A I'opposé, le patron $(1,12)$, les groupes de soutien $(1,66)$ (ex:L.O.G.E.Q.) et les comissaires industriels $(1,78)$ sont considérés comme les personnes ressources les moins actives lors de l'élaboration d'un tel projet.

Ces personnes ressources peuvent aider l'entrepreneur dans ses démarches afin d'identifier les ressources financières, materielles ou humaines, pour le supporter et l'encourager ou encore lui fournir des informations sur les affaires et/ou le secteur d'activités. Les résultats présentés au tableau 21 indiquent que la famille dans $81,3 \%$ des cas et les amis dans $71,9 \%$ des cas supportent et encouragent l'entrepreneur lors de l'élaboration du projet d'entreprise. Les amis $(43,8 \%)$ sont également consultés pour obtenir de l'information sur les affaires et/ou le secteur d'activités. De plus, 40,6\% des entrepreneurs s'adressent au personnel des institutions financières et $28,1 \%$ a la famille pour l'obtention de ressources monétaires. On observe également que les comptables $(53,1 x)$ et les avocats et notaires $(40,6 x)$ sont souvent consultes pour obtenir de l'information sur les affaires et/ou le secteur d'activités de l'entreprise. Finalement, les entrepreneurs tentent également d'obtenir du support et de l'encouragement auprés de leur future clientèle $(46,9 x)$. Par ailleurs, les entrepreneurs s'adressent généralement à la famille $(25,0 \times)$ et aux fournisseurs $(25,0 \times)$ pour l'obtention des ressources matérielles. Concernant les ressources humaines, les entrepreneurs sollicitent généralement l'aide de la famille $(34,4 \times)$, des collegues de travail $(28,1 x)$ et des amis $(28,1 x)$. 
Tableau 20

Degré de participation des personnes ressources

lors de l'elaboration du projet d'entreprise

\begin{tabular}{|c|c|c|c|c|c|}
\hline & $\mathbf{N}$ & $\bar{x}$ & $\sigma$ & $M D$ & Mo \\
\hline Famille & 32 & 3,41 & 1,37 & 3,50 & 5,00 \\
\hline Anis personnels & 32 & 3,15 & 1,27 & 3,00 & 3,00 \\
\hline Clients & 32 & 2,63 & 1,43 & 3,00 & 1,00 \\
\hline Comptables & 31 & 2,53 & 1,34 & 3,00 & 3,00 \\
\hline $\begin{array}{l}\text { Personnel d'institutions } \\
\text { financieres }\end{array}$ & 31 & 2,44 & 1,56 & 2,00 & 1,00 \\
\hline Collègue de travail & 32 & 2,22 & 1,56 & 1,50 & 1,00 \\
\hline Fournisseurs & 31 & 2,16 & 1,55 & 1,00 & 1,00 \\
\hline Centre d'aide aux entreprises & 31 & 2,09 & 1,47 & 1,00 & 1,00 \\
\hline Fonctionnaires & 31 & 2,03 & 1,53 & 1,00 & 1,00 \\
\hline Avocats ou notaires & 30 & 1,91 & 1,42 & 1,00 & 1,00 \\
\hline Commissaires industriels & 30 & 1,78 & 1,56 & 1,00 & 1,00 \\
\hline Groupe de soutien (ex: S.l.J.) & 30 & 1,66 & 1,45 & 1,00 & 1,00 \\
\hline Patron & 30 & 1,12 & 0,71 & 1,00 & 1,00 \\
\hline Autres gens d'affaires & 31 & 1,87 & 1,45 & 1,00 & 1,00 \\
\hline Autres professionnels & 30 & 1,19 & 0,83 & 1,00 & 1,00 \\
\hline
\end{tabular}

\subsubsection{Les choix strategiques}

Les choix stratégiques effectués par les entrepreneurs en phase de prédémarrage sont présentés au tableau 22. Ces choix concernent le marché, la clientele, la technologie utilisé, les caracteristiques du service, son prix, sa distribution et sa promotion. Les choix stratégiques des entrepreneurs, à l'exception du marché visé, ont eté identifiés à 
Tableau 21

Raisons invoquées par les entrepreneurs pour contacter les personnes ressources lors de la phase de prédémarrage

\begin{tabular}{|c|c|c|c|c|c|c|c|c|c|c|}
\hline & \multicolumn{2}{|c|}{$\begin{array}{l}\text { RESS. } \\
\text { FINANC. }\end{array}$} & \multicolumn{2}{|c|}{$\begin{array}{l}\text { RESS. } \\
\text { MATER. }\end{array}$} & \multicolumn{2}{|c|}{$\begin{array}{l}\text { RESS. } \\
\text { HUM. }\end{array}$} & \multicolumn{2}{|c|}{$\begin{array}{l}\text { SUPPORT } \\
\text { ENCOUR. }\end{array}$} & \multicolumn{2}{|c|}{$\begin{array}{l}\text { INFORM. } \\
\text { AFFAIRES } \\
\text { ET SECT. }\end{array}$} \\
\hline & $\mathbf{N}$ & $x$ & $\mathbf{N}$ & $\mathbf{x}$ & $\mathbf{N}$ & $x$ & $\mathbf{N}$ & $x$ & $\mathbf{N}$ & $x$ \\
\hline Famille & 9 & 28,1 & 8 & 25,0 & 11 & 34,4 & 26 & 81,3 & 10 & 31,3 \\
\hline Collegue de travail & 1 & 3,1 & 3 & 9,4 & 9 & 28,1 & 7 & 21,9 & 9 & 28,1 \\
\hline Patron & -- & --- & 1 & 3,1 & 1 & 3,1 & 2 & 6,3 & 2 & 6,3 \\
\hline Amis personnels & 3 & 9,4 & 4 & 12,5 & 9 & 28,1 & 23 & 71,9 & 14 & 43,8 \\
\hline Pers.institutions & & & & & & & & & & \\
\hline financières & 13 & 40,6 & -- & --- & 1 & 3,1 & 2 & 6,3 & 8 & 25,0 \\
\hline Avocats ou notaires & 1 & 3,1 & -- & --- & 2 & 6,3 & 6 & 18,8 & 13 & 40,6 \\
\hline Comptables & 4 & 12,5 & 1 & 3,1 & -- & --- & 5 & 15,6 & 17 & 53,1 \\
\hline Fonctionnaires & 3 & 9,4 & 2 & 6,3 & 1 & 3,1 & 3 & 9,4 & 8 & 25,0 \\
\hline Fournisseurs & -- & --- & 8 & 25,0 & -- & --- & 4 & 12,5 & 8 & 25,0 \\
\hline Clients & 1 & 3,1 & -- & --- & 2 & 6,3 & 15 & 46,9 & 10 & 31,3 \\
\hline $\begin{array}{l}\text { Commissaires indust. } \\
\text { Centre d'aide }\end{array}$ & 2 & 6,3 & 2 & 6,3 & 4 & 12,5 & 5 & 15,6 & 4 & 12,5 \\
\hline aux entreprises & 4 & 12,5 & 1 & 3,1 & 1 & 3,1 & 2 & 6,3 & 6 & 18,8 \\
\hline Groupe S.I.J. & 4 & 12,5 & 1 & 3,1 & 2 & 6,3 & 2 & 6.3 & 3 & 9,4 \\
\hline Autres gens affaires & -- & --- & 1 & 3,1 & 1 & 3,1 & 2 & 6,3 & 4 & 12,5 \\
\hline Autres professionnels & -- & --- & -- & --- & 1 & 3,1 & 2 & 6,3 & 3 & 9,4 \\
\hline
\end{tabular}


Tableau 22

Les choix stratégiques des entrepreneurs

lors de la phase de prédémarrage

N $\quad \mathbf{x}$

MARCHE

Local

1134,4

Régional

1340,6

Provincial

$4 \quad 12,5$

National

13,1

international

SCORE

1

23

45

$\mathbf{x}$

x $\quad x$

$\mathbf{x}$

$x$

$\bar{x}$

Qualité du serv. (inf./supérieure)

$-$

Clientele (masse/ciblée)

15,6

$---$

21,9

12,5

59,

4,40

Service (traditionnel/innovateur)

15,6

$3,1 \quad 18,8$

12,5

43,8

3,70

Marché (nouveau/existant)

21,9

$6,3 \quad 18,8$

15,6

34,4

3,40

Gamme/services (peu/très étendue)

15,6

$12,5 \quad 9,4$

9,4

37,5

3,20

Image/clientele (pop./prestige)

21,9

$15,6 \quad 34,4$

12,5

15,6

2,97

Distribution (exclusive/intensive)

25,0

$9,4 \quad 37,5$

6,3

18,8

2,90

Technologie (peu/trés sophist.)

31,3

$12,5 \quad 25,0$

12,5

18,8

2,87

18,8

$9,4 \quad 18,8$

12,5

21,9

2,83

Promotion (faible/elevé)

18,8

$15,6 \quad 40,6$

12,5

6,3

2,70

Prix (faible/élevé)

18,8

$15,6 \quad 43,8$

15,6

2,60

Marché (peu/beaucoup concurrence)

Nature du serv. (spec./genéral.)

43,8

$37,5 \quad 15,6$

6,3

12,5

2,43

$15,6 \quad 25,0 \quad \cdots \quad 9,4 \quad 2,10$ 
l'aide d'une échelle en cinq (5) points sur laquelle le répondant indiquait les caractéristiques spécifiques de ses orientations stratégiques lors de la formulation de son projet d'entreprise lex: $1=$ peu de concurrence et $5=$ beaucoup de concurrencel. Ainsi, la majoritédes entreprises de notre echantillon visent un marché local $(34,4 \%)$ ou régional $(40,6 x)$. De plus, 56,3 $\%$ de ces entreprises visent un marché où la concurrence est faible. Quant à l'image de la clientele, $21,9 \%$ des futurs dirigeants d'entreprises visent une clientele dite populaire, 18,8\% une clientele de prestige et $37,5 \%$ une clientèle qui se situe a mi-chemin entre les deux catégories précédentes. Par ailleurs, 43,8\% des entrepreneurs intérrogés cherchent à atteindre un clientèle ciblée et $59,4 x$ des répondants se considerent comme des spécialistes dans leur service. Une proportion de 31,3\% de ces entreprises misaient sur une technologie peu sophistiqué alors que $21,9 \%$ d'entre elles préféraient utiliser une technologie trés sophistiquee. Concernant les services à offrir, $50 x$ des entrepreneurs rencontrés désiraient desservir leur clientèle avec un service innovateur et $34,4 \times$ avec une gamme de service moyennement étendue. La stratégie prédominante consiste à offrir un service de qualité superieure à celle des competiteurs puisque $71,9 \%$ des entrepreneurs privilegient cette stratégie. Par ailleurs, 43,8x des répondants misent sur une stratégie de prix comparables à celle de leurs concurrents. Quant à la distribution, on observe que les entrepreneurs semblent privilégier autant la distribution exclusive que la distribution intensive. Finalement, 40,6x des entrepreneurs interrogés utiliseront la promotion avec une intensité comparable à celle des concurrents. Bref, dans les entreprises de services en phase de prédemarrage, les entrepreneurs misent principalement sur des services de qualité supérieure qu'ils pourront offrir a des prix comparables à ceux de leurs concurrents. De plus, ils privilégient un service innovateur et spécialisé destiné à une clientele ciblee et ce, dans un marché où la concurrence est faible. 


\subsection{VERIFICATION DES HYPOTHESES DE RECHERCHE}

Dans cette section, nous présentons les résultats obtenus concernant les comportements de gestion des entrepreneurs lies a l'analyse des environnements interne, sectoriel et général. Ces résultats specifiques ont pour objectif de verifier les hypotheses formulées dans le cadre de cette étude descriptive.

\subsubsection{Vérification de l'hypothese 1}

Selon cette hypothese, les entrepreneurs analysent à des degrés significativement différents les environnements interne, sectoriel et genéral. Ainsi, cette hypothese a pour objectif de démontrer que les entrepreneurs n'accordent pas le meme intéret, en terme d'analyse, aux environnements interne, sectoriel et général. Globalement, les résultats présentés au tableau 23, indiquent que les scores moyens obtenus concernant le degré d'analyse des environnements interne (3,45), sectoriel (3,56) et général $(3,15)$ sont différents. De plus, les tests statistiques effectués (Tests $T$ de Student) nous révelent que ces différences sont significatives dans deux (2) de ces cas seulement, soit entre les environnements interne et genéral ainsi qu'entre les envi ronnements sectoriel et général puisque les scores obtenus sont inferieurs au seuil de signification de 0,05 . Toutefois, la difference entre les environnements interne et sectoriel s'est avéré non significative puisque le score obtenu est superieur au seuil de signification de 0,05 .

De façon détaillée, le score moyen obtenu pour l'environnement interne $(3,45)$ est inférieur au score moyen de l'environnement sectoriel $(3,56)$. Cependant, le test statistique effectué (test T) nous révèle que 
Tableau 23

Conparaison du degré d'analyse des

environnements général, sectoriel et interne

\begin{tabular}{|c|c|c|c|c|c|}
\hline & $\mathbf{N}$ & $\mathbf{X}$ & $\sigma$ & Diff. & Signif. \\
\hline \multirow[t]{2}{*}{ Environnement sectoriel } & 32 & 3,56 & 0,72 & & \\
\hline & & & & 0,117 & 0,367 \\
\hline Environnement interne & 32 & 3,45 & 0,85 & & \\
\hline \multirow[t]{2}{*}{ Environnement général } & 32 & 3,15 & 0,83 & & \\
\hline & & & & $-0,297$ & 0,027 \\
\hline Environnement interne & 32 & 3,45 & 0,85 & & \\
\hline \multirow[t]{2}{*}{ Environnement général } & 32 & 3,15 & 0,83 & & \\
\hline & & & & $-0,414$ & 0,013 \\
\hline Environnement sectoriel & 32 & 3,56 & 0,72 & & \\
\hline
\end{tabular}

cette différence $(0,1167)$ est non significative puisque la probabilite obtenue (0,367) est superieure au seuil de signification de 0,05. Par ailleurs, le score moyen obtenus pour l'environnement interne $(3,45)$ est superieur au score moyen de l'environnement général $(3,15)$ et le test statistique effectue (test $T$ ) nous indique que cette différence $(-0,297$ ) est significative puisque le score $(0,027)$ est inférieur au seuil de signification de 0,05 . Enfin, le score moyen de l'environnement sectoriel $(3,56)$ est supérieur au score moyen de l'environnement général $(3,15)$ et le test statistique réalisé (test $T$ ) nous indique que cette différence ($0,414)$ est significative puisque la probabilité obtenus $(0,013)$ est inférieure au seuil de signification de 0,05 . 
En résumé, lors de la phase de prédemarrage, les entrepreneurs interrogés analysent à des degrés differents les environnements interne et général ainsi que les environnements sectoriel et général. Toutefois, ces entrepreneurs semblent accorder le même intérêt à l'analyse de l'environnement interne qu'à l'analyse de l'environnement sectoriel. Par consequent, cette hypothese a l'effet que les entrepreneurs analysent $a$ des degrés différents les environnements interne, sectoriel et général est partiellement confirmée.

\subsubsection{Vériflcation de l'hypothése 2}

Selon cette hypothèse, les entrepreneurs analysent à des degrés significativement différents chacune des principales composantes de l'environnement interne. Bref, cette hypothese cherche à démontrer que les entrepreneurs n'accordent pas le meme intéret en terme d'analyse aux composantes suivantes de l'environnement interne: le marketing, les operations, les ressources humaines et les ressources financieres. Les résultats présentés au tableau 24 démontrent que le marketing obtient le score moyen le plus élevé $(3,76)$ de l'ensemble de ces composantes de l'environnement interne. De plus, la difference entre le score moyen marketing et le score moyen des composantes opérationnelles, humaines et financieres est significative dans tous les cas puisque le score résultant des tests $T$ est inférieur au seuil de signification 0,05 . Par conséquent, on peut affirmer que les entrepreneurs analysent à un degrésignigicativement plus élevé les aspects marketing si on les compare aux aspects opérationnels, financiers et humains. Par ailleurs, le degré d'analyse des aspects operationnels se traduit par un score moyen de 3,51 ce qui est supérieur au score moyen des aspects financiers $(3,33)$ et humains $(3,21)$. Cependant, seule la différence obtenue entre les aspects 
Tableau 24

Comparaison entre le degré d'analyse

des différents aspects de l'environnement interne

\begin{tabular}{|c|c|c|c|c|c|}
\hline & $\mathbf{N}$ & $\bar{x}$ & $\sigma$ & Diff. & Signif. \\
\hline Marketing & 32 & 3,76 & & \multirow{2}{*}{0,254} & \multirow{2}{*}{0,001} \\
\hline Opération & 32 & 3,51 & 0,89 & & \\
\hline Marketing & 32 & 3,76 & 0,84 & \multirow{2}{*}{0,548} & \multirow{2}{*}{0,000} \\
\hline Huma in & 32 & 3,21 & 1,06 & & \\
\hline Marketing & 32 & 3,76 & 0,84 & \multirow{2}{*}{0,425} & \multirow{2}{*}{0,001} \\
\hline Finance & 32 & 3,33 & 0,97 & & \\
\hline Opération & 32 & 3,51 & 0,89 & \multirow{2}{*}{0,302} & \multirow{2}{*}{0,017} \\
\hline Huma i $n$ & 32 & 3,21 & 1,06 & & \\
\hline Operation & 32 & 3,51 & 0,89 & \multirow{2}{*}{0,179} & \multirow{2}{*}{0,086} \\
\hline Finance & 32 & 3,33 & 0,97 & & \\
\hline Humain & 32 & 3,21 & 1,06 & \multirow{2}{*}{$-0,123$} & \multirow{2}{*}{0,454} \\
\hline Finance & 32 & 3,33 & 0,97 & & \\
\hline
\end{tabular}

opérationnels et humains $(0,302)$ s'est avérée significative avec un score (0,017) inférieur au seuil de signification de 0,05. Ainsi, l'on peut observer que la composante opérationnelle est analysée à un degré significativement plus elevé que la composante humaine. Toutefois, la différence entre le degré d'analyse des composantes opérationnelle et fi- 
nancière s'est avérée non significative avec un score 10,086$)$ supérieur au seuil de signification 0,05. Finalement, le score moyen obtenu pour la composante financière $(3,33)$ est supérieur à celui de la composante humaine $(3,21)$. Cependant, les tests effectues nous ont permis de constater que cette différence $(-0,123)$ était non significative puisque le score obtenu $(0,454)$ est supérieur au seuil de signification 0,05 .

Par conséquent, ces resultats confirment partiellement notre hypothese soutenant que la différence entre le degré d'analyse des composantes marketing, operationnelle, financiere et humaine est significative. Bref, on observe que le marketing se démarque des quatre (4) autres composantes et la composante opérationelle de la composante financierre. Cependant, la différence entre la composante operationnel le et humaine n'est pas significative de meme que la différence entre la composante humaine et financiere.

Par ailleurs, une analyse détaillée des différents facteurs liés a chacune des composantes de l'environnement interne montre que l'ensemble des facteurs ne sont pas analysés avec le meme interet par l'entrepreneur. A cet égard, les résultats aux tests $T$ concernant les facteurs de la composante marketing (tableau 25) indiquent que la qual i té du service $(4,50)$ obtient la moyenne la plus élevee et que ce score est significativement différent de chacun des scores moyens des autres facteurs marketing etudiés. De plus, les scores moyens concernant la gamme de services à offrir $(4,09)$ et le service à la clientele $(4,10)$ sont significativement différents de ceux obtenus par la plupart des autres facteurs marketing analysés. Bref, les entrepreneurs semblent accorder plus d'interet à ces trois (3) facteurs (qualité du service, gamme de services et service à la clientelel lors de la phase de prédémarage. 
Tableau 25

Comparaison entre le degré d'analyse des différents facteurs des aspects marketing [Tests T]

\begin{tabular}{|c|c|c|c|c|c|c|c|c|c|c|}
\hline & $\begin{array}{l}\text { Gamme } \\
\text { services } \\
\text { à ofirir } \\
\bar{X}=4,09\end{array}$ & $\begin{array}{l}\text { Qualité } \\
\text { services } \\
\text { à oftrir } \\
\bar{X}=4,50\end{array}$ & $\begin{array}{c}\text { Uniformi- } \\
\text { sation } \\
\text { services } \\
\bar{X}=3,79 \\
\end{array}$ & $\begin{array}{l}\text { Capacte } \\
\text { product } \\
\text { services } \\
\bar{X}=3,5 ?\end{array}$ & $\begin{array}{l}\text { Services } \\
\text { ala } \\
\text { dientèle } \\
x=4,10\end{array}$ & $\begin{array}{c}\text { Fromotion } \\
\text { et } \\
\text { Publicité } \\
\bar{X}=3,41\end{array}$ & $\begin{array}{c}\text { Sources } \\
\text { d'iniorma } \\
\text { tions } \\
\bar{x}=3,13 \\
\end{array}$ & $\begin{array}{l}\text { Délejs de } \\
\text { liyraison } \\
\bar{X}=3,77\end{array}$ & $\begin{array}{l}\text { Accessi- } \\
\text { bilité aux } \\
\text { services } \\
\bar{X}=3,68\end{array}$ & $\begin{array}{c}\text { Fossibilite } \\
\text { de dével } \\
\text { nouvserv } \\
\bar{X}=3,41\end{array}$ \\
\hline $\begin{array}{l}\text { Prixides } \\
\text { services } \\
\text { sotfir } \\
\bar{x}=3,34\end{array}$ & 0,187 & 0,001 & 0,897 & 0,405 & 0,470 & 0,051 & 0,014 & 1,000 & 0,405 & 0,114 \\
\hline $\begin{array}{l}\text { Gammes de } \\
\text { services } \\
\text { à oftmi } \\
\bar{x}=4,09\end{array}$ & ntrkt & 0,001 & 0,161 & 0,028 & 0,839 & 0,001 & 0,000 & 0,130 & 0,030 & 0,004 \\
\hline $\begin{array}{l}\text { Qualité des } \\
\text { services } \\
\text { affrir } \\
\bar{X}=4,50\end{array}$ & nim & trmt & 0,002 & 0,000 & 0,043 & 0,000 & 0,000 & 0,003 & 0,000 & 0,000 \\
\hline $\begin{array}{l}\text { Urifomisat } \\
\text { tion des sery. } \\
\text { oftert } \\
\bar{X}=3,79\end{array}$ & 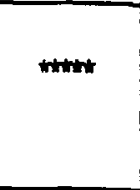 & mtrt & thtr & 0,490 & 0,043 & 0,110 & 0,011 & 0,839 & 0,363 & 0,186 \\
\hline $\begin{array}{l}\text { Capacité de } \\
\text { production } \\
X=3,57\end{array}$ & $\mathrm{mms}$ & nim & 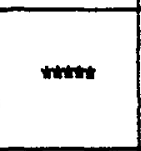 & mont & 0,031 & 0,362 & 0,020 & 0,424 & 1,000 & 0,452 \\
\hline $\begin{array}{l}\text { Service à la } \\
\text { clientèle } \\
X=4,10\end{array}$ & ntrith & numb & twht & 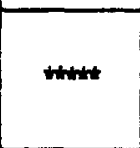 & nthoth & 0,008 & 0,001 & 0,083 & $0,0,04$ & 0,031 \\
\hline $\begin{array}{l}\text { Promotion ef } \\
\text { Fublicite } \\
\bar{X}=3,41\end{array}$ & 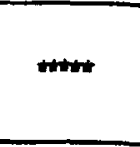 & thotrt & 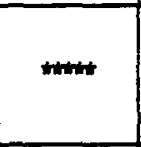 & thrth & montent & trit & 0,142 & 0,063 & 0,206 & 1,000 \\
\hline $\begin{array}{l}\text { Sources } \\
\text { intormations } \\
\bar{X}=3,13\end{array}$ & thent & thrth & $n$ & ththt & trithr & ntrmt & trith & 0,032 & 0,011 & 0,309 \\
\hline $\begin{array}{l}\text { Délais de } \\
\text { liyraison } \\
\bar{X}=3.77\end{array}$ & 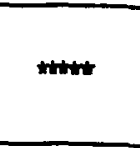 & nomit & twht & ntwr & mt & ntwr & mit & the & 0,257 & 0,123 \\
\hline $\begin{array}{l}\text { Accessibilité } \\
\text { aux services } \\
\bar{X}=3,68\end{array}$ & trint & wht & thenthe & rwht & throkt & tritht & ntrot & 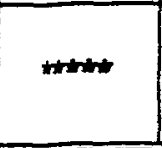 & wtm & 0,393 \\
\hline
\end{tabular}


Concernant la composante opérationnelle, le besoin en equipement et fourniture obtient le score moyen le plus elevé de 4,13 et les résultats aux tests $T$ (tableau 26 ) montrent des différences significatives en comparaison à l'ensemble des facteurs analysés sauf dans le cas des criteres de contrôle de la qualité. Bref, on observe que les entrepreneurs analysent principalement leur besoin en equipement et fourniture en ce qui concerne la dimension operationnelle.

Pour la composante humaine, les résultats (tableau 27 ) indiquent que les scores moyens obtenus concernant les qualifications requises $(4,03)$ et les tăches a executer $(3,78)$ enregistrent des ecarts significatifs avec l'ensemble des autres énoncés. Enfin, on constate que l'intérêt des entrepreneurs est concentré principalement sur ces deux (2) variables lors de l'analyse de la composante humaine.

Les résultats concernant la composante financière (tableau 28) montre que les entrepreneurs analysent à des degrés comparables l'ensemble des variables liées à la fonction finance, c'est-à-dire que les scores obtenus (tests $T$ ) sont généralement non significatifs.

En résumé, ces résultats spécifiques montrent que les variables liées aux différentes composantes de l'environnement interne ne sont pas tous analysées avec le même intérêt par les entrepreneurs lors de la phase de prédémarrage, dans les entreprises de services. Par ailleurs, on observe que dans le cas des composantes marketing, opérationnelle et humaine, certaines variables sont plus analysées par l'entrepreneur, ce qui ne semble pas etre le cas pour la fonction financière. 
Tableav 26

Comparaison entre le degré d'analyse des différents facteurs de l'aspect opérationnel [Tests T]

\begin{tabular}{|c|c|c|c|c|c|c|c|c|c|c|c|}
\hline & $\begin{array}{c}\text { Besoins } \\
\text { ressour- } \\
\text { ces } \\
\bar{x}=3,18\end{array}$ & $\begin{array}{l}\text { Stanuar- } \\
\text { disation } \\
\text { méthode } \\
\text { travail } \\
\bar{x}=3,66\end{array}$ & $\begin{array}{c}\text { Citeres } \\
\text { de } \\
\text { sélection } \\
\text { toumit } \\
\bar{x}=3,19\end{array}$ & $\begin{array}{l}\text { Niveau } \\
\text { de stock } \\
\text { foumit } \\
\bar{X}=3,40\end{array}$ & $\begin{array}{c}\text { Coufs } \\
\text { d'opera } \\
\text { tion } \\
\bar{x}=3,47\end{array}$ & $\begin{array}{l}\text { Cnteres } \\
\text { de loca } \\
\text { lisation } \\
\bar{x}=3,20\end{array}$ & $\begin{array}{l}\text { Cuteres } \\
\text { sélection } \\
\text { equipe- } \\
\text { ments } \\
\dot{x}=3,72\end{array}$ & $\begin{array}{l}\text { Ament } \\
\text { gement } \\
\text { physique } \\
\bar{x}=3,75\end{array}$ & $\begin{array}{l}\text { Ontére } \\
\text { contrôle } \\
\text { qualité } \\
\bar{x}=3,78\end{array}$ & $\begin{array}{c}\text { Gestion } \\
\text { des } \\
\text { achats } \\
\bar{X}=3,28\end{array}$ & $\begin{array}{l}\text { Fentica } \\
\text { ton \& } \\
\text { contôle } \\
\text { gperat } \\
x=3,41\end{array}$ \\
\hline $\begin{array}{l}\text { Besoins equip } \\
8 \text { fournitures } \\
\mathrm{x}=4,13\end{array}$ & 0,000 & 0,025 & 0,000 & 0,000 & n.1013 & 0,003 & 0,013 & 0,050 & 0,133 & 0,000 & 0,002 \\
\hline $\begin{array}{l}\text { Besoins en } \\
\text { ressources } \\
\bar{X}=3,18\end{array}$ & thoth & 0,070 & 0,699 & 0,397 & 0,107 & 0,885 & 0,051 & 0,013 & 0,019 & 0,379 & 0,234 \\
\hline $\begin{array}{l}\text { Standardisa } \\
\text { tion méthode } \\
\text { trayail } \\
\bar{X}=3,66 \\
\end{array}$ & ntrth & throt & 0.037 & 0,335 & 0,516 & 0,166 & 0.786 & 0,707 & 0,587 & 0,083 & 0,234 \\
\hline $\begin{array}{l}\text { Citere selec- } \\
\text { tion foumitures } \\
R=3,19\end{array}$ & thrtr & thit & thint & 0,264 & 0,293 & 0,908 & 0,027 & 0,022 & 0,005 & 0,752 & 0,229 \\
\hline $\begin{array}{l}\text { Niveau de } \\
\text { stock toumit } \\
\bar{x}=3,40\end{array}$ & wht & thenth & trit & 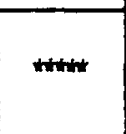 & 0,655 & 0,433 & 0,076 & 0,046 & 0,009 & 0,227 & 1,500 \\
\hline $\begin{array}{l}\text { Côuts } \\
\text { d'opération } \\
\bar{x}=3,47\end{array}$ & 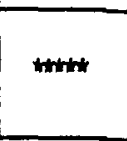 & wht & 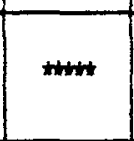 & whth & whrth & 0,257 & 0,211 & 0,119 & 0,186 & 0,281 & 0,778 \\
\hline $\begin{array}{l}\text { Criteres de } \\
\text { iocalisation } \\
\bar{X}=3,20\end{array}$ & 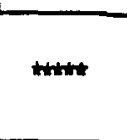 & 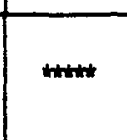 & Hurt & 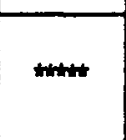 & 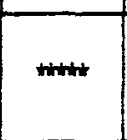 & 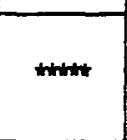 & 0,024 & 0,015 & 0,030 & 0,537 & 0,318 \\
\hline $\begin{array}{l}\text { Criteres } \\
\text { sélection } \\
\text { équipements } \\
\mathrm{Q}=3,72\end{array}$ & throw & witht & ntrit & ntwt & mot & ntrm & nntrots & 0.851 & 0,790 & 0,020 & 0,177 \\
\hline $\begin{array}{l}\text { Amenagement } \\
\text { physique } \\
Z=3,75\end{array}$ & Htht & whrth & nimist & nntw & nimint & wht & ntrth & thint & 0,876 & 0,016 & 0,086 \\
\hline $\begin{array}{l}\text { Critere contróle } \\
\text { gualité } \\
x=3,78\end{array}$ & twiter & 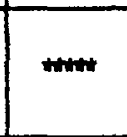 & 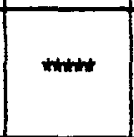 & twht & whrot & thitrit & 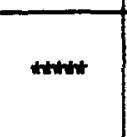 & nhrot & tה & 0,001 & 0,003 \\
\hline $\begin{array}{l}\text { Gestion des } \\
\text { achats } \\
\vec{Q}=3,28\end{array}$ & Hith & ntht & tht & 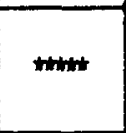 & 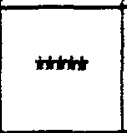 & whtrt & nintrit & 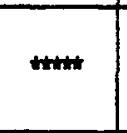 & th & ththt & 0,110 \\
\hline
\end{tabular}


Tableau 27

Comparaison entre le degré d'analyse des diftérents facteurs des aspects humains [Tests $\Pi$ ]

\begin{tabular}{|c|c|c|c|c|c|c|c|c|c|c|c|c|c|}
\hline & $\begin{array}{c}\text { Tatres } \\
\dot{y} \\
\text { gréued } \\
\bar{x}=3,78\end{array}$ & $\begin{array}{l}\text { Moyens } \\
\text { coond } \\
\text { activites } \\
\bar{x}=3,31\end{array}$ & $\begin{array}{l}\text { Dusifi } \\
\text { Cations } \\
\text { requises } \\
\bar{x}=4,63\end{array}$ & $\begin{array}{c}\text { Remus } \\
\text { rétion } \\
\bar{x}=3,44\end{array}$ & $\begin{array}{l}\text { Wéthode } \\
\text { recute- } \\
\text { ment } \\
\bar{x}=2,65\end{array}$ & $\begin{array}{l}\text { Citeres } \\
\text { setectican } \\
\bar{x}=285\end{array}$ & $\begin{array}{l}\text { Arcuel } \\
\text { nounct } \\
\text { Employe } \\
\bar{x}=2,83\end{array}$ & $\mid \begin{array}{c}\text { Exigences } \\
S S T \\
\bar{X}=2,78\end{array}$ & $\begin{array}{l}\text { Bescoins } \\
\text { iomation } \\
\bar{x}=3.10\end{array}$ & 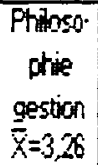 & $\mid \begin{array}{l}\text { Methode } \\
\text { d'évelua- } \\
\text { lign pess. } \\
\vec{X}=258\end{array}$ & $\begin{array}{l}\text { céanom } \\
\text { dun } \\
c A \\
\bar{x}=2.26\end{array}$ & $\begin{array}{l}\text { Utisation } \\
\text { consellers } \\
\text { itemes } \\
\bar{x}=3,00\end{array}$ \\
\hline $\begin{array}{l}\text { Besoirs R.H. } \\
x=323\end{array}$ & 0,002 & 7!IIII) & ग. JoT & 0,580 & 0,059 & 0,153 & 0,236 & 0,117 & 0,476 & 0,905 & 0,008 & 0006 & 0,355 \\
\hline $\begin{array}{l}\text { Tíntes ì } \\
\text { extecules } \\
\bar{X}=3,78\end{array}$ & 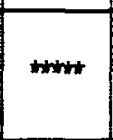 & 0,001 & 0,103 & 0,054 & 0,001 & 0,001 & 0,003 & 0,003 & 0,006 & 0,013 & 0,000 & 0,005 & 0,005 \\
\hline $\begin{array}{l}\text { Hoyens coord } \\
\text { activites } \\
B=3,31\end{array}$ & thromt & whth & 0,100 & 0,514 & 0,020 & 0,175 & 0,224 & 0,204 & 0,326 & 0,614 & 0,007 & 0,048 & 0,274 \\
\hline $\begin{array}{l}\text { Quaffications } \\
\text { Iequises } \\
x=4,03\end{array}$ & ntwt & thent & whth & 0,005 & 0,000 & 0,000 & 0,011 & 0,003 & 0,001 & 0,001 & 0,000 & 0,001 & 0,000 \\
\hline $\begin{array}{l}\text { Rémunef acion } \\
\chi=3,44\end{array}$ & $7+$ & thenthe & thitht & 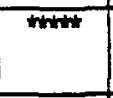 & 0,029 & 0,123 & $\overline{0,240}$ & 0,138 & 0,332 & 0,380 & 0,032 & 0,061 & 0,114 \\
\hline $\begin{array}{l}\text { Méthodes de } \\
\text { Iecuternent } \\
\bar{X}=2,65\end{array}$ & nhint & $w+t$ & whth & ththt & thrth & 0,409 & 0,487 & 0,740 & 0,338 & 0,059 & 0,704 & 10,864 & 1,407 \\
\hline $\begin{array}{l}\text { Critères siect } \\
\bar{X}=2,25\end{array}$ & thenthents & 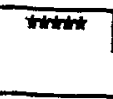 & trith & timint & 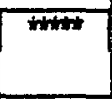 & thint & 0,753 & 0,917 & 0,558 & 0,211 & 0,186 & 0,546 & 0,814 \\
\hline $\begin{array}{l}\text { Accuei nous. } \\
\text { emplosé } \\
X=2,83\end{array}$ & $n+t$ & wht & thot & 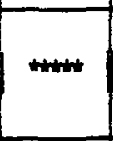 & 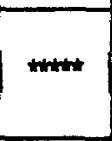 & 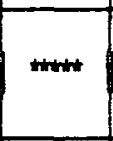 & 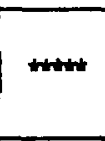 & 0,784 & 0.732 & 0,059 & 0,231 & 0,669 & 0,812 \\
\hline \begin{tabular}{|l|} 
Exigences \\
SST \\
$X=2,78$ \\
\end{tabular} & whith & ththt & thrtht & $n$ & ntwr & whtt & thtrt & twh & 0,775 & 0,115 & 0,709 & 0,172 & 1,000 \\
\hline $\begin{array}{l}\text { Besoin en } \\
\text { lomation } \\
\bar{x}=3,10\end{array}$ & twt & 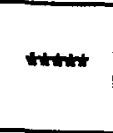 & nothet & nowot & nimit & noth & 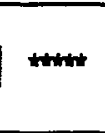 & 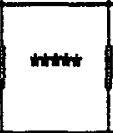 & 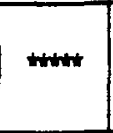 & 0,732 & 0,103 & 0,067 & 0,802 \\
\hline $\begin{array}{l}\text { Phiosophie } \\
\text { de gostion } \\
X=3,26\end{array}$ & tht & ththt & thintit & with & thith & whtt & thitht & nhith & thitht & norrit & 0,011 & 0,219 & 0,573 \\
\hline $\begin{array}{l}\text { Méthode } \\
\text { déval pers. } \\
\bar{X}=2,58\end{array}$ & retrots & thtst & זيt & whtst & thtst & thttrt & tht & trits & tht & thith & rtrots & 0,645 & 0,288 \\
\hline $\begin{array}{l}\text { Ciézation din } \\
C . A \\
X=2,26 \\
\end{array}$ & thint & 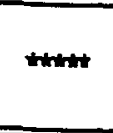 & ntrtt & thint & wht & thit & thith & thith & thtst & tht & throtht & thrt & 0,367 \\
\hline
\end{tabular}


Tableau 28

Comparaison entre le degré d'analyse des différents

facteurs des aspects financiers (Tests $T$ ]

\begin{tabular}{|c|c|c|c|c|c|c|c|c|c|c|}
\hline & $\begin{array}{l}\text { Capacite } \\
\text { endette- } \\
\text { ment } \\
\bar{X}=3,43\end{array}$ & $\begin{array}{l}\text { Côts } \\
\text { globaux } \\
\bar{x}=3,69\end{array}$ & $\begin{array}{c}\text { Chiffe } \\
\text { d'afraires } \\
\text { potertiel } \\
\bar{x}=3,31\end{array}$ & $\begin{array}{l}\text { Point } \\
\text { mont } \\
\bar{X}=3,41\end{array}$ & $\begin{array}{c}\text { Pertes ou } \\
\text { protits } \\
\text { potertiels } \\
X=3,13\end{array}$ & $\begin{array}{c}\text { Variation } \\
\text { ronds } \\
\text { roulement } \\
\bar{x}=3,06\end{array}$ & $\begin{array}{c}\text { Taxes et } \\
\text { impôt } \\
\text { ìpayer } \\
\bar{x}=3,25\end{array}$ & $\begin{array}{l}\text { Proc de } \\
\text { contôle } \\
\text { financier } \\
\bar{x}=3,28\end{array}$ & $\begin{array}{c}\text { Eudgets } \\
\text { prevision- } \\
\text { nels } \\
\bar{x}=3,00\end{array}$ & $\begin{array}{l}\text { Sources } \\
\text { de finsn- } \\
\text { dement } \\
x=3,32\end{array}$ \\
\hline $\begin{array}{l}\text { Besuins de } \\
\text { tonde } \\
\bar{x}=3,72\end{array}$ & 0,083 & 0,839 & 0,068 & 0.201 & 0,003 & 0,014 & 0,074 & 0,129 & 0,017 & $0,17 \overline{6}$ \\
\hline $\begin{array}{l}\text { Capacité } \\
\text { endettement } \\
x=343\end{array}$ & norrert & 0,048 & 0,752 & 0,662 & 0,153 & 0,051 & 0,282 & 0,423 & 0,056 & 0,475 \\
\hline $\begin{array}{l}\text { Couts } \\
\text { globaux } \\
x=3,69\end{array}$ & 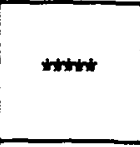 & 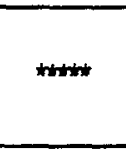 & 0,083 & 0,194 & 0,010 & 0,011 & 0,075 & 0,079 & 0,0018 & 0,231 \\
\hline $\begin{array}{l}\text { Chilfes d'aff. } \\
\text { potentiel } \\
\mathrm{X}=3.31\end{array}$ & trt & 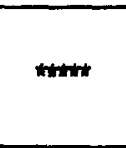 & ntrt & 0,638 & 0,226 & 0,187 & 0,829 & 0,889 & 0,096 & 0,889 \\
\hline $\begin{array}{l}\text { Point } \\
\text { mort } \\
X=3,41\end{array}$ & thrt & thrth & wht & thrth & 0,071 & 0,025 & 0,556 & 0,501 & 0,030 & 0,897 \\
\hline $\begin{array}{l}\text { Pertes/profits } \\
\text { potentiels } \\
\mathrm{X}=3,13\end{array}$ & thrt & thrth & thrth & twth & nwrt & 0,690 & 0,645 & 0,475 & 0,546 & 0,255 \\
\hline $\begin{array}{l}\text { Yariation } \\
\text { fonds roul. } \\
\bar{x}=3,06\end{array}$ & thrt & 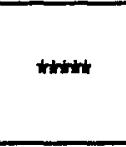 & mintre & mont & ntrt & 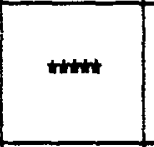 & 0,469 & 0,243 & 0,712 & 0,178 \\
\hline $\begin{array}{l}\text { Taxes \& } \\
\text { impôt à payen } \\
\vec{X}=3,25\end{array}$ & trint & tht & 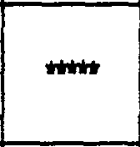 & 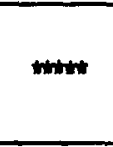 & wrtht & 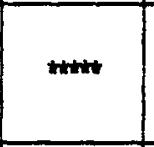 & thrnt & 0,901 & 0,391 & 0,687 \\
\hline $\begin{array}{l}\text { Procedures } \\
\text { de contôle } \\
\text { financier } \\
\bar{X}=3,28\end{array}$ & thent & nntw & ntrt & m & 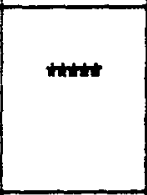 & nintrr & 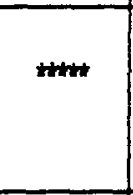 & mot & 0,095 & 0,897 \\
\hline $\begin{array}{l}\text { Budgets } \\
\text { previsionnels } \\
\mathrm{X}=3,00\end{array}$ & 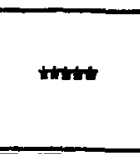 & $\mathrm{mm}$ & $\mathrm{mint}$ & tritrt & thtrt & ntrot & $m+m$ & $m+t$ & wrrt & 0,125 \\
\hline
\end{tabular}




\subsubsection{Vérification de l'hypothese 3}

Selon cette hypothèse, les entrepreneurs analysent à des degrés $\mathbf{s}$ gniflcativement différents chacune des dimensions de l'environnement sectoriel. Bref, cette hypothese cherche a démontrer que les entrepreneurs n'accordent pas le meme intéret, en' terme d'analyse, aux dimensions suivantes de l'environnement sectoriel: les disponibilités en ressources, les clients, les fournisseurs et les concurrents. Les résultats présentés au tableau 29 indiquent que les differences entre chacune des dimensions etudiés sont non significatives puisque les scores résultants des tests $T$ sont dans tous les cas superieurs au seuil de signification 0,05 . Bref, on observe que les entrepreneurs analysent à des degrés comparables l'ensemble des dimensions de l'environnement sectoriel. Par consequent, cette hypothese doit etre rejetée dans sa totalite.

Par ailleurs une analyse détaillée des différents facteurs liés a chacune des dimensions de l'environnement sectoriel montre que l'ensemble des facteurs ne sont pas analysés avec le meme interet par les entrepreneurs, lors de la phase de prédemarrage. A cet égard, les résultats concernant la dimension disponibilité en ressources (tableau 30 ) révelent que la disponibilite en ressources humaines obtient le score moyen le moins élevé et que la différence entre ce score et la moyenne des autres facteurs de cette dimension est significative dans tous les cas. Ainsi, les résultats obtenus auprés de notre echantillon d'entreprises de services indiquent que la disponibilité des ressources humaines est un facteur moins préoccupant pour les entrepreneurs lors de l'analyse de la disponibilite des ressources.

Quant a la dimension clients (tableau 31), les resultats indiquent que l'ensemble des facteurs sont analysés à des degrés di férents puisque 
Tableau 29

\section{Comparaison entre le degré d'analyse des différentes dimensions de l'environnement sectoriel}

\begin{tabular}{|c|c|c|c|c|c|}
\hline & $\mathbf{N}$ & $\bar{x}$ & $\sigma$ & Diff. & Signif. \\
\hline Disp. ressources & 26 & 3,51 & & \multirow{2}{*}{0,163} & \multirow{2}{*}{0,461} \\
\hline Clients & 26 & 3,35 & 0,85 & & \\
\hline Disp. ressources & 26 & 3,51 & & \multirow{2}{*}{$-0,177$} & \multirow{2}{*}{0,565} \\
\hline Fournisseurs & 26 & 3,69 & 0,98 & & \\
\hline Disp. ressources & 26 & 3,51 & 1,20 & \multirow{2}{*}{$-0,123$} & \multirow{2}{*}{0,660} \\
\hline Concurrents & 26 & 3,64 & 0,73 & & \\
\hline Clients & 32 & 3,55 & 0,78 & \multirow{2}{*}{$-0,010$} & \multirow{2}{*}{0,211} \\
\hline Fournisseurs & 32 & 3,65 & 0,10 & & \\
\hline Clients & 32 & 3,55 & 0,78 & \multirow{2}{*}{$-0,036$} & \multirow{2}{*}{0,792} \\
\hline Concurrents & 32 & 3,59 & 0,88 & & \\
\hline Fournisseurs & 32 & 3,65 & 0,10 & \multirow{2}{*}{0,064} & \multirow{2}{*}{0,711} \\
\hline Concurrents & 32 & 3,59 & 0,88 & & \\
\hline
\end{tabular}

les tests $T$ montrent des différences significatives dans tous les cas, à l'exception du couple de facteurs ouverture de marché autres provinces $(1,67)$ et possibilité d'exportation hors Canada $(1,00)$. Ces résultats révelent que les entrepreneurs s'intéressent davantage aux besoins de la clientele $(4,38)$ qu'aux autres facteurs soit, en ordre decroissant:l'identification des types de clientèle $(3,97)$, la taille du marché (3,63), 
Tableau 30

\begin{abstract}
Comparaison entre le degré d'analyse des différents facteurs
llés a la disponibilité des ressources
\end{abstract}

\begin{tabular}{lcc}
\hline & $\begin{array}{c}\text { Disponibilité } \\
\text { equipements } \\
(\bar{X}=4,29)\end{array}$ & $\begin{array}{c}\text { Disponibilité } \\
\text { prod. \& fourn. } \\
(\bar{X}=4,10)\end{array}$ \\
\hline $\begin{array}{l}\text { Disponibilite } \\
\text { ress. humaines } \\
(\bar{X}=3,03)\end{array}$ & 0,000 & 0,000 \\
\hline $\begin{array}{l}\text { Disponibilite } \\
\text { equipements } \\
(\bar{X}=4,29)\end{array}$ & 0,394 \\
\hline
\end{tabular}

les caractéristiques du territoire visé $(3,19)$ et de façon équivalente, l'ouverture des marchés autres provinces $(1,67)$ et la possibilitéd'exportation hors Canada $(1,00)$.

Pour la dimension fournisseurs, les résultats des tests $T$ (tableau 32) indiquent que, généralement, les entrepreneurs analysent à des degrés comparables l'ensemble des facteurs a l'étude puisqu'on obtient des différences non significatives dans la majorité des cas.

Les résultats obtenus pour la dimension concurrents (tableau 33 ), montrent que les entrepreneurs n'accordent pas le meme interet, en terme d'analyse, à tous les facteurs qui y sont rattachés. De facon génerale, les scores moyens des facteurs services substituts $(2,63)$ et possibilite 
Tableau 31

Comparaison du degré d'analyse des differents

facteurs liés à la dimension clients

\begin{tabular}{|c|c|c|c|c|c|}
\hline & $\begin{array}{l}\text { Ident. } \\
\text { types } \\
\text { client. } \\
(\bar{x}=3,97)\end{array}$ & $\begin{array}{l}\text { Taille } \\
\text { marché } \\
(\bar{X}=3,63)\end{array}$ & $\begin{array}{l}\text { Ouvert. } \\
\text { marché } \\
\text { autres } \\
\text { prov. } \\
(\bar{X}=1,67)\end{array}$ & $\begin{array}{l}\text { Possib. } \\
\text { export. } \\
\text { hors } \\
\text { Canade } \\
(\bar{x}=1,00)\end{array}$ & $\begin{array}{l}\text { Caract. } \\
\text { territoire } \\
(\bar{x}=3,19)\end{array}$ \\
\hline $\begin{array}{l}\text { Besoin de la } \\
\text { clientele } \\
(\bar{x}=4,38)\end{array}$ & 0,013 & 0,002 & 0,000 & 0,000 & 0,000 \\
\hline $\begin{array}{l}\text { Identification } \\
\text { types clienteles } \\
(\bar{X}=3,97)\end{array}$ & ---- & 0,039 & 0,000 & 0,000 & 0,000 \\
\hline $\begin{array}{l}\text { Taille du } \\
\text { marche } \\
(\bar{X}=3,63)\end{array}$ & $-\cdots$ & ---- & 0,000 & 0,000 & 0,026 \\
\hline $\begin{array}{l}\text { Ouverture marché } \\
\text { autres provinces } \\
(\bar{X}=1,67)\end{array}$ & $-\cdots$ & $-\cdots$ & $-\cdots$ & 0,136 & 0,001 \\
\hline $\begin{array}{l}\text { Possibilité } \\
\text { d'exportation } \\
\text { hors Canada } \\
(\bar{X}=1,00)\end{array}$ & $-\cdots$ & $-\cdots$ & $-\cdots$ & $-\cdots$ & 0,000 \\
\hline
\end{tabular}


Tableau 32

Comparaison entre le degré d'analyse des différents facteurs de la dimension fournisseurs (Tests $T$ )

\begin{tabular}{|c|c|c|c|c|c|c|c|c|}
\hline & \begin{tabular}{|c|}
$\begin{array}{c}\text { Localisation } \\
\text { des foum. } \\
\overline{\mathrm{X}}=3, \overline{81}\end{array}$ \\
\end{tabular} & $\begin{array}{l}\text { Caract } \\
\text { produits \& } \\
\text { services } \\
\bar{X}=3,81\end{array}$ & $\begin{array}{l}\text { Prix des } \\
\text { produits } \\
\bar{x}=3,74\end{array}$ & $\begin{array}{l}\begin{array}{l}\text { Délais de } \\
\text { lirraison }\end{array} \\
\vec{X}=3,63\end{array}$ & $\begin{array}{c}\begin{array}{c}\text { Quantite } \\
\text { disporible }\end{array} \\
\bar{X}=3,52\end{array}$ & $\begin{array}{c}\text { Qualite des } \\
\text { services } \\
\bar{X}=3.84\end{array}$ & $\begin{array}{c}\text { Termes de } \\
\text { crédit } \\
\text { consentis } \\
\bar{X}=3,25\end{array}$ & $\begin{array}{l}\text { Wolonté de } \\
\text { faje affaires } \\
\text { ensemble } \\
\bar{X}=3,45\end{array}$ \\
\hline $\begin{array}{l}\text { Nombre de } \\
\text { foumisseurs } \\
X=3,63\end{array}$ & 0,344 & 0,380 & 0,466 & 0,663 & 0,655 & 0,200 & 0,030 & $0,6,39$ \\
\hline $\begin{array}{l}\text { Localisation des } \\
\text { foumisseurs } \\
x=3,31\end{array}$ & twrontr & 0,856 & 0,647 & 0,444 & 0,118 & 1,000 & 0,019 & 0,077 \\
\hline $\begin{array}{l}\text { Caracteristiques } \\
\text { produits'services } \\
\bar{X}=3,81\end{array}$ & thrtrt & thent & 0,745 & 0,620 & 0,326 & 0,882 & 0,021 & 0,133 \\
\hline $\begin{array}{l}\text { Frix des produits } \\
\text { et services } \\
\square=3,74\end{array}$ & ntwr & trimb & tritst & 0,752 & 0,255 & 0,655 & 0,085 & 0,071 \\
\hline $\begin{array}{l}\text { Délais de } \\
\text { livraison } \\
X=3,63\end{array}$ & norter & not & ntrt & mtrt & 0,206 & 0,455 & 0,161 & 0,326 \\
\hline $\begin{array}{l}\text { Quantité } \\
\text { disponible } \\
\chi=3,52\end{array}$ & nowt & thentht & nowntr & thet & ntront & 0,155 & 0,408 & 0,865 \\
\hline $\begin{array}{l}\text { Qualité des } \\
\text { services } \\
\bar{X}=3,84\end{array}$ & new & whth & ntint & whit & trumert & trithrt & 0,013 & 0,110 \\
\hline $\begin{array}{l}\text { Termes de } \\
\text { crédit consentis } \\
\bar{X}=3,25\end{array}$ & 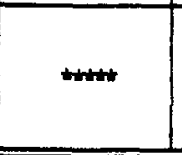 & 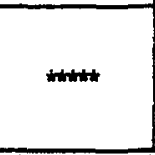 & tht & 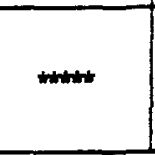 & trontort & 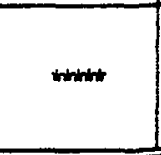 & 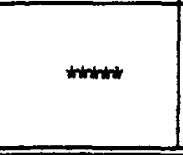 & 0,621 \\
\hline
\end{tabular}


Tableau 33

Comparaison entre le degré d'analyse des différents facteurs de la dimension concurrents (Tests $\Pi$

\begin{tabular}{|c|c|c|c|c|c|c|c|c|c|c|}
\hline & $\begin{array}{l}\text { Localisat } \\
\text { tion des } \\
\text { concur. } \\
\bar{x}=4,00\end{array}$ & $\begin{array}{l}\text { Fart de } \\
\text { marché } \\
\text { concur. } \\
\bar{X}=3,59 \\
\end{array}$ & $\begin{array}{l}\text { Carad } \\
\text { services } \\
\text { concur. } \\
\bar{X}=3,80\end{array}$ & $\begin{array}{l}\text { Prix des } \\
\text { serwices } \\
\text { concur. } \\
\mathrm{X}=3,94 \\
\end{array}$ & $\begin{array}{c}\text { Qualite } \\
\text { des sert } \\
\text { concur. } \\
\bar{y}=4,00\end{array}$ & $\begin{array}{c}\text { Distribu- } \\
\text { fion } \\
\text { concur. } \\
\bar{X}=3.44 \\
\end{array}$ & $\begin{array}{c}\text { Fublicté } \\
\text { promotion } \\
\text { concur. } \\
\bar{X}=3,39\end{array}$ & $\begin{array}{l}\text { Services } \\
\text { substituts } \\
\overline{\mathrm{X}}=2,63\end{array}$ & $\begin{array}{l}\text { Possibilité } \\
\text { nouveaul } \\
\text { concur. } \\
\bar{X}=2,74 \\
\end{array}$ & $\begin{array}{l}\text { Forces et } \\
\text { iajblessed } \\
\text { ouncur. } \\
\bar{X}=3,50 \\
\end{array}$ \\
\hline $\begin{array}{l}\text { Nombre de } \\
\text { concuments } \\
x=3,31\end{array}$ & 0,735 & 0,238 & 0.893 & 0.827 & 0,608 & 0,185 & 0,092 & 0,001 & 0,001 & 0,297 \\
\hline $\begin{array}{l}\text { Localisation } \\
\text { Concuments } \\
X=4,00\end{array}$ & mrmet & 0,030 & 0,407 & 0,901 & 0,884 & 0,126 & 0,026 & 0,001 & 0,000 & 0,250 \\
\hline $\begin{array}{l}\text { Part de } \\
\text { miarche } \\
\text { concur. } \\
\bar{x}=3,59\end{array}$ & 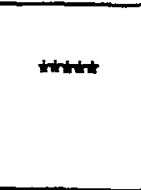 & 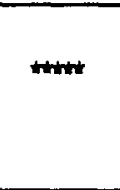 & 0,184 & 0,110 & 0,055 & 1,000 & 0,501 & 0,015 & 0,001 & 0,925 \\
\hline $\begin{array}{l}\text { Caradéris- } \\
\text { tiques sery. } \\
\text { concur. } \\
\bar{Q}=3,80\end{array}$ & trttt & nwht & thrort & 0,378 & 0,090 & 0.216 & 0,031 & 0,002 & 0,001 & 0,433 \\
\hline $\begin{array}{l}\text { Pix des } \\
\text { sery concur. } \\
X=3,94\end{array}$ & nown & ntwit & thth & wht & 0,625 & 0,024 & 0,007 & 0,001 & 0,000 & 0,155 \\
\hline $\begin{array}{l}\text { Gualité des } \\
\text { serv. concur. } \\
\bar{X}=4,00\end{array}$ & 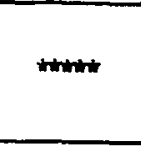 & nontrot & ntrotrt & thot & nomtrt & 0,001 & 0,001 & 0,000 & 0,000 & 0,040 \\
\hline $\begin{array}{l}\text { Distribution } \\
\text { concurrents } \\
X=3,44\end{array}$ & ntht & nntrt & ntm & whint & wnt & trmt & 0,833 & 0,056 & 0,097 & 0,398 \\
\hline $\begin{array}{l}\text { Publicité el } \\
\text { promotion } \\
\text { concur. } \\
\bar{x}=3,39 \\
\end{array}$ & nomont & thenturt & thenthrt & trotht & thtwt & ntwht & throwt & 0,014 & 0,005 & 0,325 \\
\hline $\begin{array}{l}\text { Services } \\
\text { substituts } \\
\bar{X}=2,63\end{array}$ & 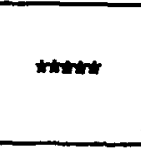 & mit & 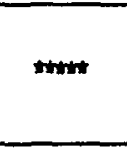 & trist & ntrit & nrmt & 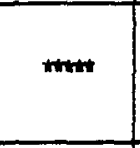 & mome & 0,404 & 0,001 \\
\hline $\begin{array}{l}\text { Possibilité } \\
\text { d'arivée de } \\
\text { now concur. } \\
\bar{X}=2.74\end{array}$ & ththt & whit & ntorth & 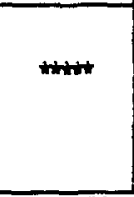 & ntw & montont & thrmt & 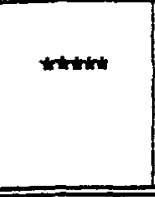 & ntwht & 0,001 \\
\hline
\end{tabular}


d'arrivée de nouveaux concurrents $(2,74)$ sont significativement différents des scores moyens de la plupart des autres facteurs. Il semble donc que les entrepreneurs en phase de prédemarrage analysent avec un moindre intérét ces deux (2) facteurs (services substituts et possibilité d'arrivee de nouveaux concurrents). Par contre, les autres facteurs lies a la concurrence semblent intéresser a des degrés equivalents les entrepreneurs.

Bref, ces résultats indiquent que lors de la phase de prédémarrage, les entrepreneurs n'analysent pas avec le meme intéret les variables liés à chacune des dimensions de l'environnement sectoriel. Ainsi, on observe que pour les dimensions disponibilité des ressources et concurrents, les facteurs les moins analyses sont mis en évidence. Par ailleurs, la dimension clients demontre certaines particularités puisque tous les facteurs sont analyses à des degrés différents à l'exception des variables ouverture de marchés autres provinces et possibilités d'exportation hors Canada. Quant à la dimension fournisseurs, tous les facteurs sont analysés avec le même intéret par les entrepreneurs.

\subsubsection{Vérification de l'hypothése 4}

Selon cette hypothése, les entrepreneurs analysent à des degrés significativement différents chacun des facteurs de l'envi ronnement géneral. Bref, cette hypothèse cherche à demontrer que les entrepreneurs n'accordent pas le même intéret, en terme d'analyse, aux différentes variables composant l'environnement général. Les résultats des tests $T$ (tableau 34 ) indiquent que le score moyen de la valeur du dollar canadien $(2,16)$ comparé aux scores moyens de la plupart des autres variables enregistrent des différences significatives. Ceci tend à démontrer que les 
Tableau 34 Comparaison entre le degré d'analyse des différents
facteurs de l'environnement général (Tests T)

\begin{tabular}{|c|c|c|c|c|c|c|c|c|c|c|c|c|c|c|c|c|}
\hline & $\begin{array}{l}\text { कon } \\
\text { tom } \\
x=3,48\end{array}$ & $\begin{array}{l}\text { Tas } \\
\text { frt } \\
x=3,10\end{array}$ & $\begin{array}{l}\text { Tasi } \\
\text { dint } \\
\bar{x}=26 ?\end{array}$ & $\begin{array}{l}\text { yaleus } \\
\text { dolls } \\
\text { canad. } \\
x=2.16\end{array}$ & $\begin{array}{l}\text { Aormes } \\
\text { ention } \\
\text { rement } \\
\bar{x}=3,63\end{array}$ & $\begin{array}{l}\text { Ternos- } \\
\text { ratues is } \\
\text { sassons } \\
x=3,56\end{array}$ & $\begin{array}{l}\text { Ten } \\
\text { dince } \\
\text { deno. } \\
x=2,33\end{array}$ & $\begin{array}{l}\text { lotino } \\
\text { logie } \\
\text { flispon. } \\
x=3.31\end{array}$ & $\begin{array}{l}\text { Sibu. } \\
\text { goin. } \\
\bar{x}=3,15\end{array}$ & $\begin{array}{l}\text { Pinde } \\
\text { gown. } \\
x=2,81\end{array}$ & $\begin{array}{l}1501 \\
\bar{x}=265\end{array}$ & $\begin{array}{l}\text { Lot } \\
\text { murici } \\
p \text { jes } \\
x=2.81\end{array}$ & $\begin{array}{l}\text { Lois } \\
\text { bätiment } \\
\bar{x}=3.044\end{array}$ & $\begin{array}{l}\text { S.nte } \\
\text { tugiere } \\
\bar{x}=3,38\end{array}$ & $\begin{array}{c}\text { Mames } \\
d y \\
\text { daya } \\
x=3,3\end{array}$ & $\begin{array}{l}\text { Fiscate } \\
\bar{x}=3,61\end{array}$ \\
\hline $\begin{array}{l}\text { con } \\
\text { econ } \\
x=3,59\end{array}$ & 0.331 & 0,069 & 0,013 & 0,001 & 0,697 & 0,875 & 0,006 & 0,333 & 0,412 & 0,073 & 0,005 & 0.043 & 0,148 & 0,505 & 0,534 & 0.917 \\
\hline 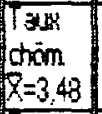 & $x=\pi x$ & 0,317 & 0,077 & 0,010 & 0,837 & 0,670 & 0,65 & 0,633 & 0,583 & 0.374 & 0,078 & 0,331 & 0,856 & 1,000 & 0.704 & 0,643 \\
\hline $\begin{array}{l}\text { Tax } \\
\text { orteset } \\
=3,10\end{array}$ & 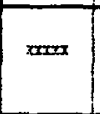 & $x$ & 0,025 & 0,013 & 0,399 & 0,416 & 0,398 & 0,045 & 0.506 & 0,571 & 0.114 & 0.575 & 0,901 & 0,770 & 0,403 & 0,268 \\
\hline $\begin{array}{l}\text { sut } \\
\text { onhat } \\
x=2,67\end{array}$ & $x$ & $\operatorname{Ix}$ & $\operatorname{mex}$ & 0,052 & 0,223 & 0.150 & 0,493 & 0,040 & 0,186 & 0,490 & 0,899 & 0,642 & 0,053 & 0,183 & 0,056 & 0,023 \\
\hline $\begin{array}{l}\text { odeu } \\
5 \text { can } \\
x=2,16\end{array}$ & חשר & $=$ & $x=x$ & $=$ & 0,075 & $0,0 \mathrm{~m}$ & 0,131 & 0,102 & 0,014 & 0,053 & 0,168 & 0,017 & 0,003 & 0,037 & 0,002 & 0,001 \\
\hline $\begin{array}{l}\text { Nomes } \\
\text { envion } \\
x=3,63\end{array}$ & $=0$ & XI & $\operatorname{mox}$ & $\operatorname{mox}$ & $\pi$ & 0,418 & 0,208 & $n, 86$ & 0,363 & 0,326 & 0,167 & 0,288 & 0,293 & 0,883 & 0,902 & 0.669 \\
\hline $\begin{array}{l}\text { emp. } \\
\text { saisor } \\
X=3,56\end{array}$ & $x$ & 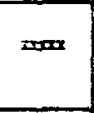 & $\operatorname{rrm}$ & $+x$ & 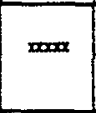 & $x$ & 0,000 & 0,300 & 0,552 & 0,317 & 0,024 & 0,106 & 0,354 & 0,714 & 0,842 & 0,733 \\
\hline $\begin{array}{l}\text { Tend } \\
\text { demo. } \\
x=2,30\end{array}$ & $\max$ & $\pi$ & (1) & 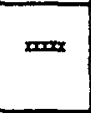 & 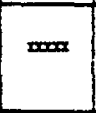 & 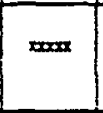 & 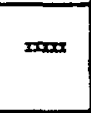 & 0.118 & 0,033 & 0,064 & 0.691 & 0,288 & 0,059 & 0,227 & 0,025 & 0.007 \\
\hline $\begin{array}{l}\text { Tetmo } \\
\text { disp. } \\
x=3,31\end{array}$ & $x$ & 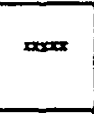 & ס & $\operatorname{mox}$ & 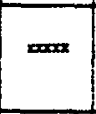 & $=$ & $\mathbf{m}$ & $=x$ & 0,606 & 0.179 & 0.229 & 0,247 & 0,452 & 0,736 & 1,000 & 0,409 \\
\hline $\begin{array}{l}\text { Subv. } \\
\text { gave. } \\
x=3,15\end{array}$ & $\infty$ & $\infty$ & $\max$ & $\max$ & $x$ & 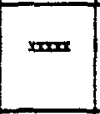 & Imx & $x$ & 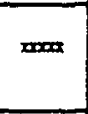 & 0,148 & 0,138 & 0,191 & 0,237 & 0,758 & 0,905 & 0,262 \\
\hline $\begin{array}{l}\text { fode } \\
\text { gown. } \\
X=2,81\end{array}$ & $x$ & $\sin$ & (10x) & $x$ & 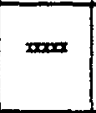 & (2000 & 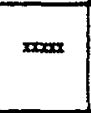 & 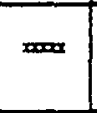 & 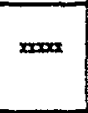 & $x=x x$ & 0,312 & 0,815 & 1,000 & 0,404 & 0,437 & 0,044 \\
\hline $\begin{array}{l}\text { CST } \\
X=2,65\end{array}$ & مוס & $\max$ & זמש & m & (1) & $\operatorname{mox}$ & (x) & 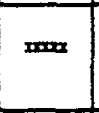 & $\max$ & $\boldsymbol{m}$ & $x$ & 0.802 & 0.493 & 0.120 & 0,019 & 0.016 \\
\hline $\begin{array}{l}\text { Dos } \\
\text { indicic } \\
X=2.81\end{array}$ & $\infty$ & 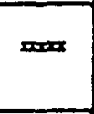 & $x$ & ס्ल & (1) & 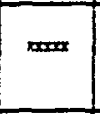 & $x$ & x) & 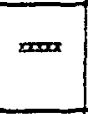 & Inxx & 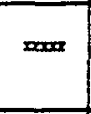 & 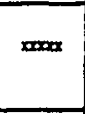 & 0,491 & 0,008 & $0,0,036$ & 0,030 \\
\hline $\begin{array}{l}\text { Lots } \\
\text { bätinen } \\
X=3,04\end{array}$ & 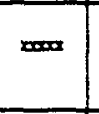 & 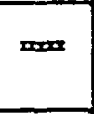 & $\operatorname{xn} x$ & 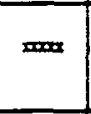 & $\operatorname{mox}$ & 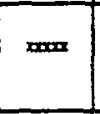 & max & $x=x$ & $x$ & $\operatorname{sex}$ & $\operatorname{roxx}$ & 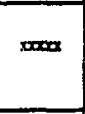 & x- & 0.216 & 0,236 & 0,233 \\
\hline $\begin{array}{l}\text { Sante } \\
\text { hygiene } \\
x=3,38\end{array}$ & nam & 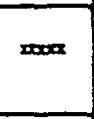 & $\operatorname{mxx}$ & $x$ & 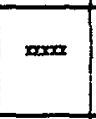 & ס & $\pi$ & $x$ & 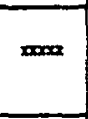 & $m$ & $x$ & $x=0$ & $x=$ & 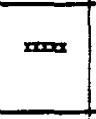 & 0,755 & 0,781 \\
\hline $\begin{array}{l}\text { Nomes } \\
\text { troval } \\
x=3,38\end{array}$ & max & 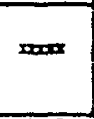 & Ints & 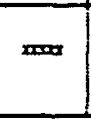 & $m$ & 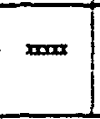 & זמס & x & xax & $x$ & 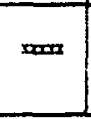 & $x$ & $=0 \times$ & x) & $m$ & 0.504 \\
\hline
\end{tabular}


125

entrepreneurs analysent avec moins d'intéret la valeur du dollar canadien que la plupart des autres facteurs a l'etude. Ce résultat pourrait etre lía à la nature locale et régionale du marché visé. Par allleurs, les autres facteurs semblent analysés à des degrés comparables d'intéret par les entrepreneurs en phase de prédémarrage puisque les tests $T$ enregistrent des différences non significatives dans la majorité des cas. Par conséquent, cette hypothèse à l'effet que les entrepreneurs analysent à des degrés significativement différents les variables de l'environnement général est partiellement confirmée. 
CHAPITRE $V$

DISCUSSION ET INTERPRETATION DES RESULTATS 
CHAPITRE IV

\section{DISCUSSION ET INTERPRETATION DES RESULTATS}

Les résultats de la présente étude nous fournissent des informations intéressantes concernant les comportements de planification stratégique des entrepreneurs lors de la phase de prédémarrage et sur certaines des caractéristiques de l'échantillon. De façon générale, nous observons que les entrepreneurs intérrogés sont plus ăgés et plus scolarisés que ceux rencontrés dans d'autres etudes s'intéressant à l'entrepreneurship québécois (Lorrain et Raymond, 1988; Garnier et Gasse, 1986; Belley, 1987; Dussault et Lorrain, 1987). Par ailleurs, ces entrepreneurs béneficient de plusieurs années d'expérience sur le marché du travail et ce, principalement en contexte de PME. Ce résultat vient supporter les dires de certains auteurs à l'effet que les PME constituent d'excellents incubateurs d'entrepreneurs (Lorrain, 1990; Garnier et Gasse, 1986; Belley, 19871. En outre, l'influence de la famille, caractéristique identifiee dans la littérature, semble jouer un rôle important auprés des entrepreneurs à l'étude puisque la majorité d'entre eux ont un membre de leur famille en affaires. De plus, les résultats nous indiquent que ces entrepreneurs avaient acquis des connaissances en gestion et des connaissances du secteur d'activités de la future entreprise gráce à lour formation académique et a leur expérience de travail. Cette observation tend à demontrer que, conformement a la littérature, les entrepreneurs se lancent en affaires dans un secteur d'activités qu'ils connaissent bien. Quant aux facteurs motivant le répondant à se lancer en affaires, les résultats de cette étude viennent appuyer la litterature puisque les besoins d'accomplissement, d'indépendance et d'autonomie ont été identifiés comme étant les principales sources de motivation de l'entrepreneur 
(Belley, 1987). Par ailleurs, les résultats nous révelent que les entrepreneurs rencontres sont généralement responsables de l'ensemble des activites de l'entreprise, ce qui vient appuyer la littérature qui considere l'omnipresence du propriétaire dirigeant comme une des caractéristiques principales des PME. Bref, les caractéristiques psycho-sociologiques décrivant cet echantillon de créateurs d'entreprises de services sont comparables à celles observées dans d'autres études à l'exception de l'äge et de la scolarité.

Concernant la phase de prédemarrage, nous obtenons également des résultats fort intéressants. D'une part, il semble que la majorité des entrepreneurs interrogés consacrent peu de temps à la préparation de leur projet d'entreprise, ce qui peut s'expliquer par l'age, la scolarite et le niveau de connaissances du secteur d'activités qui semblent plus élevés chez les entrepreneurs rencontrés. Lors de cette préparation, la majorite des entrepreneurs de l'échantillon ont elaboré un plan d'affaires et se sont fixés des objectifs à court terme. Cette dernière constatation est conforme aux résultats obtenus dans d'autres études demontrant que la planification stratégique dans les PME est principalement orientee sur le court terme (Stoner, 1983; Shuman et Seeger, 1986). Les résultats de cette recherche indiquent egalement que les futurs dirigeants d'entreprises de services avaient précisé les variables stratégiques motrices définissant la mission de l'organisation (service, territoire visé, clientele visée et modes d'operationl. D'autre part, la vérification du niveau de connaissances des entrepreneurs relativement à la gestion et au secteur d'activités indiquent que les répondants étaient genéralement bien informes et ce, particulièrement en ce qui concerne certains facteurs de l'environnement sectoriel, soit: les principaux concurrents, les principaux fournisseurs et les clients. Ce résultat est légerement supérieur à celui obtenu par Dussault et Lorrain (1987) avec un echantil- 
Ion d'entreprises manufacturières en phase de prédemarrage. Par contre, les entrepreneurs semblent eprouver certaines difficultés concernant l'évaluation du volume de ventes potentielles. Pourtant, cette donnee constitue une information capitale pour demontrer la valeur de l'opportunité d'affaires que le futur entrepreneur désire exploiter.

En reférence au contenu proposé en matière de planification stratégique et de plan d'affaires, la littérature en entrepreneurship recommande a tout nouvel entrepreneur désirant se lancer en affaires, qu'une analyse de tous les facteurs externes et internes à l'entreprise soit effectuee afin d'accroitre sa comprehension de la dynamique du fonctionnement de la future entreprise. Une telle demarche s'associe a l'approche systémique qui suggère à l'entrepreneur d'avoir une vision holistique de la nouvelle entité économique (Belley, Dussault et Lorrain,1989). Contrairement à ces recommandations theoriques proposées par la littérature, les résultats obtenus concernant l'étude des comportements de gestion des entrepreneurs liés à l'analyse des environnements général, sectoriel et interne lors de la phase de predémarrage montrent que les entrepreneurs interrogés n'accordent pas le meme intéret à chacun de ces environnements. Ainsi, les entrepreneurs se préoccupent davantage de leurs environnements interne et sectoriel que de leur environnement géneral. Toutefois, ils semblent accorder le meme intéret aux environnements sectoriel et interne.

Par ailleurs, les résultats indiquent que le degré d'analyse des facteurs lís à chacun des environnements général, sectoriel et interne est différent. Bien que l'environnement general soit l'environnement le moins analyse par les entrepreneurs en comparaison des environnements sectoriel et interne, on constate que l'ensemble des variables de l'environnement général sont analysées à des niveaux equivalents, à l'exception 
de la valeur du dollar canadien qui semble peu anslyé par les entrepreneurs. Toutefois, la nature locale et regionale du marché vise par ses nouvelles entreprises peut expliquer le peu d'intéret manifester à l'égard de cette dernière variable.

De plus, on constate que l'ensemble des dimensions de l'environnement sectoriel, soit: la disponibilité en ressources, les clients, les fournisseurs et les concurrents qui sont analysées avec le meme interet par l'entrepreneur. Cependant, des resultats détaillés indiquent la présence de variations dans le niveau d'analyse de certaines variables. Ainsi, on observe que la disponibilité en ressources humaines constitue le facteur le moins analyse, lors de la phase de predémarrage, en comparaison de la disponibilité en équipement et de la disponibilite en produits et fournitures. Ce résultat s'expliquerait par le faible besoin en ressources humaines associé à la création et au demarrage des entreprises de services. En rapport à la dimension clients, on observe que les entrepreneurs se concentrent principalement sur les elements besoins de la clientele, identification des types de clientéle, taille du marché ainsi que sur les caractéristiques du territoire visé. Par contre, les variables ouverture de marchés autres provinces et possibilites d'exportation hors Canada constituent des eléments peu considerées par les entrepreneurs lors des analyses. Ceci s'explique par l'orientation locale et régionale de ces entrepreneurs concernant le demarrage de la nouvel le entreprise de services. Quant à la dimension concurrents, on constate que les entrepreneurs analysent avec le même intérêt l'ensemble des variables qui y sont reliees à l'exception des variables services substituts et possibilités d'arrivée de nouveaux concurrents auxquelles ils accordent moins d'intéret. Ceci nous apparait paradoxal au fait que les entrepreneurs considèrent que les services substituts et l'arrivee de nouveaux concurrents representent des menaces importantes pour la future 
entreprise. Enfin, on observe que lors de la phase de prédémarrage, les entrepreneurs analysent a des degrés comparables l'ensemble des facteurs liés à la dimension fournisseurs.

Par ailleurs, les résultats obtenus concernant les aspects internes (les aspects marketing, operationnels, financiers et humains) indiquent que les aspects marketing sont au centre des preoccupations des entrepreneurs rencontrés et que les aspects opérationnels sont analysés avec plus d'intéret que les aspects humains. En contre partie, ces entrepreneurs accordent le meme interet, en terme d'anal yse, aux aspects opérationnels qu'aux aspects financiers ainsi qu'aux aspects financiers et humains. En parallele, les résultats obtenus par Dussault et Lorrain (1987) concernant les comportements de gestion d'entrepreneurs du secteur manufacturier en phase de demarrage indiquent qu'à ce stade de vie, l'intéret de l'entrepreneur est concentré davantage sur la fonction production. Ce qui nous amène à croire que les préoccupations majeures de l'entrepreneur concernant les fonctions de l'entreprise pourraient varier avec le stade de développement et le secteur d'activités de l'entreprise. De plus, les résultats détaillés révèlent que les degrés d'analyse des variables liées à chacune des dimensions de l'environnement interne enregistrent certaines différences. Plus spécifiquement, on observe qu'en matière de marketing, les entrepreneurs accordent beaucoup d'intérêt à la qualité des services à offrir, à la gamme de services à offrir et au service a la clientele. Concernant la dimension operationnelle, les besoins en équipements et fournitures constitue la variable la plus analysée par les entrepreneurs. Ce résultat liéd l'environnement interne est cohérent avec les résultats de l'environnement sectoriel concernant l'analyse de la disponibilité des ressources où les facteurs disponibilité en equipements et disponibilité en produits et fournitures représentaient les variables les plus analysées par les entrepreneurs. En 
matiere de ressources humaines, l'entrepreneur concentre ses analyses principalement sur les täches a executer et sur les qualifications requises alors que tous les facteurs liés à la fonction finance sont analysés à un meme niveau par les entrepreneurs, lors de la phase de predémarrage.

Bref, on observe qu'au moment de la formulation du premier plan stratégique et operationnel de l'entreprise (projet d'entreprise), tous les facteurs environnementaux ne sont pas analysés avec le meme intéret par les entrepreneurs tel que recommandé par la litterature normative en entrepreneurship. Il semble que les facteurs à proximité de l'entreprise (facteurs de l'environnement interne et sectoriel) intéressent davantage l'entrepreneur contrairement aux facteurs de l'environnement genéral. De plus, il apparaitt que l'entrepreneur considere principalement les facteurs liés au marketing lors de l'analyse de l'environnement interne, alors que toutes les composantes de l'environnement sectoriel sont évaluées de façon équivalente par l'entrepreneur lors de ce premier stade de développement de l'entreprise.

Lors de cette phase de prédemarrage, les entrepreneurs consultent diverses sources, tant personnelles qu'impersonnelles, afin de recueillir toutes les informations nécessaires à la préparation de leur projet d'entreprise. Les résultats de cette etude demontrent que les entrepreneurs recherchent principalement de l'information sur la clientele, l'économie et les concurrents. De plus, les amis, les revues et journaux ainsi que les clients sont les sources d'information privilégiés par les futurs proprietaires-dirigeants d'entreprises de services. Par ailleurs, les entrepreneurs consultés comptent généralement sur la participation de diverses personnes ressources pour les aider dans la réalisation de ce projet. La famille et les amis representent les personnes les plus actives 
lors de ces démarches et c'est vers eux que se tournent, le plus souvent, les entrepreneurs pour obtenir du support et de l'encouragement. Ces resultats sont conformes à ceux obtenus par Lorrain (1990) dans son étude sur les jeunes entrepreneurs québécois. De plus, on constate que l'entrepreneur, lors de la phase de prédémarrage, est fréquemment à la recherche d'informations sur les affaires mais aussi de support et d'encouragement et qu'a cette fin, ils contactent diverses personnes ressources.

Par ailleurs, les résultats complementaires concernant les choix strategiques des entrepreneurs lors de la phase de prédémarrage révelent que le marché visé est majoritairement local ou régional. De plus, on constate que la majorité des entrepreneurs ont des orientations stratégiques ayant comme principales caracteristiques les points suivants: on vise un marché ayant peu de concurrence, on cherche a atteindre une clientele très ciblé, on cherche a offrir un service specialise, on considere le service offert comme étant innovateur, les services offerts seront de qualité supérieure et les prix sont comparables à ceux des concurrents.

La création d'entreprise constitue un stimulant economique non négligeable dans notre société puisqu'elle génere de nombreux emplois. C'est donc dans cette perspective que cette etude a été réalisée. Bien qu'elle ne considere qu'une infime partie de la problematique des comportements de gestion des entrepreneurs en phase de prédémarrage, elle met en évidence certains faits fort intéressants. D'une part, on constate que la réalité telle que vécue par les entrepreneurs diffère de ce qui est recommandé dans la littérature. Ainsi, contrairement à ce que propose la littérature en matière de planification strategique et de plan d'affaires qui privilegie une vision holistique de l'entreprise, l'en- 
trepreneur concentre ses efforts de planification princlpalement sur l'analyse de certaines variables environnementales. II semble, en effet, que l'entrepreneur priorise l'analyse des environnements interne et sectoriel au detriment de l'environnement général. Par ailleurs, cette etude démontre que les entrepreneurs en phase de prédémarrage accordent un interêt particulier a l'analyse de la dimension marketing et qu'ils se préoccupent plus spécifiquement de la qualité des services à offrir. Cette préoccupation se réflète également au niveau des choix stratégiques puisque la qualité supérieure du service représente une orientation stratégique majeure.

Selon les auteurs en entrepreneurship, la phase de prédemarrage constitue une période preparatoire devant contribuer à la création et à la réusite de la nouvelle entreprise. Cependant, un écart important apparait entre le contenu prescrit par la litterature en matiere de planification stratégique et de plan d'affaires et les résultats obtenus dans le cadre de cette étude. Parallèlement à ce constat, une nouvelle interrogation se pose: les comportements de gestion adoptés par les entrepreneurs lors de la phase de prédémarrage et les dimensions qu'ils privilégient ont-elles une influence sur la survie de ces futures entités économiques ?

De plus, il faut aussi reconnaitre les différences de secteur d'activités qui peuvent se traduire par des exigences specifiques et influencer les comportements de planification stratégique et opérationnel le de l'entrepreneur lors de la phase de prédémarrage. Parallélement, le stade de développement constitue un autre facteur de contingence pouvant expliquer les différences de comportements de gestion des entrepreneurs. Bref, les résultats de cette etude apportent des informations interessantes mais suscitent de nouvelles interrogations: 
Quelle est l'influence des comportements de gestion adoptés par les entrepreneurs lors de la phase de prédemarrage sur la reussite de la future entreprise?

Quelle est l'influence du stade de développement d'une entreprise sur les comportements de gestion des entrepreneurs?

A cet égard, des recherches futures devront aborder la problématique de l'evolution des comportements de gestion en favorisant une approche longitudinale et multi-sectorielle. Le nombre elevé de creation d'entreprises et le taux élevé d'échecs chez ces nouvelles entreprises justifient l'interet que les chercheurs doivent apporter à la phase de prédémarrage afin d'accroltre nos connaissances.

Pour terminer, il convient de souligner les principales limites de cette recherche. Ainsi, la taille de l'échantillon (32 entreprises) representent la principale limite de cette étude, ce qui a pour effet de restreindre la validité externe de ces résultats et par conséquent, la portée de ces conclusions. Une autre limite à reconnaitre est liée à la validité et a la fidelité de l'outil de mesure utilisédans le cadre de cette recherche. Malgré ces limites méthodologiques, il n'en demeure pas moins que cette recherche descriptive fournit un apport intéressant a l'explication des comportements de planification strategique des entrepreneurs lors de la phase de prédémarrage et souligne de nouvelles avenues de recherches concernant cette problématique. 


\section{CONCLUSION}

La phase de prédemarrage d'une entreprise est une étape préparatoire pendant laquelie les futurs entrepreneurs élaborent les premiers plans stratégiques et opérationnels de l'entreprise. Cette recherche avaient comme principal objectif d'identifier et d'evaluer les comportements de planification stratégique des entrepreneurs lors de ce premier stade de vie de l'entreprise. Les résultats obtenus auprés de cet echantillon d'entreprises de services mettent en évidence des faits fort intéressants. D'une part, les participants de cet etude sont trés scolarisés et possedent une grande expérience sur le marché du travail. De plus, ces créateurs d'entreprises se lancent genéralement en affaires, dans un secteur d'activités qu'ils connaissent bien et ce, particulièrement en ce qui concerne les concurrents, les fournisseurs et les clients. D'autre part, bien que ces individus consacrent peu de temps à l'elaboration de leur projet d'entreprise, ils semblent respecter les premieres étapes du processus de planification stratégique puisqu'ils définissent la mission de l'entreprise et élaborent certains objectifs a court terme. Par ailleurs, lors de l'analyse des environnements genéral, sectoriel et interne, les futurs dirigeants d'entreprises concentrent principalement leur attention sur certaines dimensions environnementales. Ainsi, les environnements sectoriel et interne les préoccupent davantage que l'environnement genéral. Plus spécifiquement, ils s'intéressent de facon equivalente à toutes les variables de l'environnement général, à l'exception de la valeur du dollar canadien qui leur semble peu importante en raison de la nature locale et régionale du marché visé. Concernant l'environnement sectoriel, chacune des dimensions étudiées est analysée, par les entrepreneurs, à des degrés comparables. Toutefois, des disparités apparaissent au niveau de l'analyse des variables liées à chacune de ces dimensions. Quant à l'environnement interne, le marketing cons- 
titue la dimension la plus analysée par les entrepreneurs qui s'intéressent de façon tres marquée à la qualité du service à offrir. On observe également que les aspects operationnels preoccupent davantage les entrepreneurs que les aspects humains. Cependant, les aspects opérationnels et financiers de meme que les aspects financiers et humains sont analysés à des degrés comparables. De plus, on observe certaines differences entre le degré d'analyse des variables liés a chacun de ces aspects de l'environnement interne, sauf en ce qui concerne l'aspect financier où l'ensemble des variables sont analysés à des niveaux equivalents.

En complément à toute l'analyse des variables des environnements général, sectoriel et interne, on observe que les entrepreneurs rencontrés considerent la variable conjoncture économique tant comme une opportunité que comme une menace pour la future entreprise. De plus, les entrepreneurs estiment que les besoins de la clientele et le nombre de concurrents constituent des facteurs représentant une opportunité lors du démarrage de l'entreprise. En opposition, deux (2) autres menaces liés à la concurrence ont été identifiées par les entrepreneur soit, l'arrivée de nouveaux concurrents et les services substituts. Dans le meme ordre d'idée, l'analyse de l'environnement interne a permis l'identification, par les entrepreneurs, des principales forces et faiblesses pouvant favoriser ou nuire a la création de l'entreprise. Ainsi, lescaractéristiques personnelles de l'entrepreneurs, le service à offrir et la connaissance du secteur d'activités constituent des forces majeures pour ces entreprises alors que les connaissances de la gestion et les finances de l'entreprise representent les principales faiblesses identifiées par les entrepreneurs de l'étude. 
La réalisation de cette analyse environnementale de l'entreprise implique une recherche active d'information. A cette fin, les futurs dirigeants consultent genéralement leurs amis, les revues et journaux ainsi que les clients pour obtenir diverses informations, principalement en ce qui concerne la clientele, l'economie et les concurrents. La famille et les amis sont, quant a eux, les personnes ressources les plus impliquées lors de l'elaboration du projet d'entreprise et c'est principalement vers eux que ce tournent les entrepreneurs pour obtenir le support et l'encouragement recherchés.

Par ailleurs, les choix stratégiques, dans les entreprises de services en phase de prédémarrage, reposent principalement sur l'offre de services de qualité superieure. De plus, les futurs dirigeants s'orientent vers l'offre de services innovateurs et spécialisés, destinés à une clientele ciblée et ce, dans un marché où la concurrence est faible. La stratégie de prix, quant à elle, consiste à vendre leurs services à des prix equivalents a ceux des concurrents.

Bref, les comportements de planification stratégique adoptés par les entrepreneurs en phase de prédemarrage diffèrent de ceux proposés par la littérature. En effet, l'entrepreneur concentre ses efforts de planification principalement sur certaines dimensions l'environnement de la future entreprise. Cette observation suggere de nouvelles avenues de recherche qui consisteraient a verifier la relationentre le secteur d'activités et les comportements stratégiques, à vérifier l'influence des comportements stratégique sur le succes futur de l'entreprise de meme que l'influence du stade de développement de l'entreprise sur les comportements stratégiques adoptés par les entrepreneurs. Ainsi, la compréhension de cette problématique repose principalement sur la réalisation d'études longitudinales. L'accroissement des connaissances sur la 
phase de prédemarrage et sur l'influence qu'a cette dernière sur les stades de vie futurs de l'entreprise, pourrait aider les entrepreneurs dans la planification de leur projet d'entreprises et peut-être, augmenter les chances de succès de ces nouvelles entités économiques. 


\section{B IBLIOGRAPHIE}

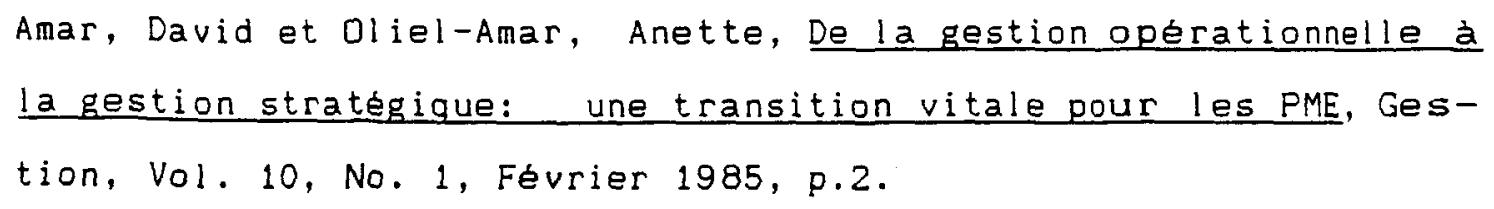

Andrew, K.R., The concept of corporate strategy, Homewood, IL: Richard D., Irein, Inc., 1980.

Ansoff, Igor H., Stratégie du développement de l'entreprise, Hommes et Techniques, France, 1974, 165 pages.

Bamberger, Ingolf, Le management stratégique dans les P.M.E., Direction et gestion des entreprises, Vol. 21, No. 4, Juillet-Aoitit $1985, p .15$.

Belley, Andre, Les milieux incubateurs, Devenez entrepreneur, Office de Planificaiton et de Développement du Québec, Québec, Aoit 1987, 106 pages.

Belley, André, Dussault, Louis et Lorrain, Jean, Le pland'établissement prototype: analyse critique du contenu de plans d'affaires, Ministère de l'agriculture, des pecheries et de l'alimentation du Québec, $1989,64 \mathrm{p}$.

Brooks, Julie K. et Stevens, Barry A., How to write a successful business plan, New York, Amacom, 1987.

Calori, Roland, Effective Strategies in Emerging Industries, Long Range Planning, Vol. 18, No. 3, 1985, p.55. 
Churchill, N.C., et Lewis, V.L., Les cing stades de l'évolution d'une PME, Harvard L'expansion, automne, 1983, pp.51-63.

Cooper, A.C., Strategic Management: new ventures and small business, voir Schendel, D.E., et Hoffer, C.W. Strategic Management: a neuw view of Manalgement policy and planning, Little Brown and Company, 1979.

Coté, Marcel et collaborateurs, La gestion stratégique d'entreprise, concept et cas, Gaëtan Morin, Québec, 1991, 1971 pages.

Cyert, R.M. et March J.G., A Behavioral Theory of the Firm, Prentice-Hall, Inc., Englewood Cliffs M.J. 1963, chap.lll; M. shubik, "Information, Risk, Ignorance and Indeterminacy,", The quaterly Journal of Economics, Vol. 68, 1954, pp. 620-640.

D'Amboise, Gérald, Planification stratégique dans les PME: des modeles émergents de la litterature, Revue P.M.O., Vol. 4, No. 2, 1989, p. 46 .

Davig, William, Business strategies in smaller manufacturing firms, Journal of Small Business Management, Janvier 1986, p.38.

Dell'aniello, Paul, Un plan d'affaires gagnant, Montréal, Publifor Inc., 1987 .

Dussault, Louis et Lorrain, Jean, Les comportements de gestion et les types d'entrepreneurs: cas d'entreprise manufacturieres en phase de démarrage, Université du Québec à Trois-Rivières, TroisRivières, 1987,132 pages. 
Fortin, Paul-A., Devenez entrepreneur, Presse de l'université Laval, Québec, 1986, 302 pages.

Fourcade, Colette, La PME dans un monde en mutation, Presse de l'Université du Québec, Québec, 1986, p.253-277

Garnier, Bernard et Gasse, Yvon, Une expérience de formation dans le domaine de l'entrepreneurship et de la création d'entreprises au Québec: Le cas du projet "Devenez entrepreneur", Université Laval, Québec, 1986,19 pages.

Gasse, Yvon, L'utilisation de diverses techniques et pratiques de gestion dans la PME, Revue P.M.0., Vol. 4, No. 1, P.3.

Gasse, Yvon, Bouchard, Marcelle et D'amours, Aline, Posséder mon entreprise, Guide pour réaliser le plan d'affaires, Ottawa, L'Institut de recherches politiques, 1988.

Gauthier, Benoit, Recherche Sociale; de la problématique à la collecte des données, Presse de l'Université du Québec, 1986, 535 pages .

Gill, John, Factors Affecting the Survival and Growth of the Smaller Company, Gower, 1985, 227 pages.

Gilmore, F., Formulating strategy in smaller companies, Harvard Business Review, Vol. 49, No 3, mai-juin, 1971, pp.71-81.

Good, Walter S., Building a dream, a comprehensive guide to starting a business of your own, Toronto, McGraw-Hill Ryerson, 1989. 
Industrie, Sciences et Technologie Canada, La petite entreprise au Canada 1991, Ministère de l'approvisionnement et services, 1991 , 69 pages.

Jauch, Lawrence R. et Glueck, William F., Management stratégique et politique générale, Mc Graw-Hill, Montréal, 1990, 465 pages.

Jones, David W., Characteristics of planning in small firms, Journal of Small Business Management, Juillet 1982, p.15.

Julien, Paul-Andre et Marchesnay, Michel, La petite entreprise, Edition G. Vermette, Ottawa, 1987, 288 pages.

Lalonde, Claude, Faillites d'entreprises: une etude en contexte régional au Québec. Thèse de doctorat, Québec, Université Laval, 1984 .

Lorrain, Jean, Les jeunes entrepreneurs quebecois, Gouvernement du Québec, Ministère de l'industrie, du Commerce et de la Technologie, GREPME, U.Q.A.T., Trois-Rivieres, 1990, 41 pages.

Lorrain, Jean et Dussault, Louis, Les entrepreneurs en phase de démarrage: profil psychologique et comportement de gestion, Revue P.M.O., Vol. 2, No. 1, 1986, p.26.

Lorrain, Jean et Raymond, Louis, Les nouvelles entreprises quebecoises 1988, Gouvernement du Québec, Ministere de l'industrie, du Commerce et de la Technologie, GREPME, U.Q.A.T., TroisRivieres, 1988,53 pages. 
Lovelock, C.H., Classifying services to gain strategic market insight, Journal of Marketing, Vol. 46, Summer 1983.

Mancuso, Joseph R., How to write a wining business plan, New York, Prentice Hall Press, 1985.

Martinet, A. Ch., Stratégie, Librairie Vulbert, Paris 1983, 320 pages.

Mayer, K. et Goldstein, S., The first two years: Problems of small firm growth and survival, Washington, D.C., Small Business Administration, 1961 .

McClaughlin,, Harold J., Building your business plan, a step by step approach, New York, John Wiley and Sons, 1985.

Miles, R.E. et Snow, C.C., Organizational Strategy, Structure and Process, New York, McGraw-Hill, 1978.

Ministere de l'Industrie, du Commerce et de la Technologie (MIC), Les PME au Québec, etat de la situation, Gouvernement du Québec, 1988,141 pages.

Mintzberg, Henry, Les organisations ont-elles besoin de stratégies?, Gestion, Vol. 12, No. 4, novembre 1987, p.5.

Moyer, Reed, Strategic planning for the small firm, Journal of Small Business Management, July 1982, p.8. 
Nagel, Strategy Formulation for the Emaller Firm: A Fractical Approach, Long-Range Planning, Vol. 14, No. 4, aoit 1981, pp.115120.

Noël, Alain, Comme un funambule..., Gestion, vol. 14, No. 3, septembre 1989 , p.10.

Organisation des Nations Unies pour le Developpement Industriel (ONUDI), Manuel de préparation des études de faisabilité industrielle, New York, John Wiley and Sons, 1978.

Perry Chad, Growth strategies for small firms: principles and case studies, International Small Business Journal, 1986, Vol. 5, No. 2, p. 17 .

Porter, Michael, L'avantage concurrentiel, comment devancer seg concurrents et maintenir son avance, InterEditions, Paris, 1986 , 647 pages.

Ragab, M., A Concept of Strategy for Smaller Business, Journal of Small Business Canada, Vol. 1, No. 1, été 1983, pp.4-9.

Robidoux, Jean, Les crises administratives dans les PME en croissance, Gaëtan Morin, 1980, 125 pages.

Robinson, R., Forecasting and the Small Business: A study of the Strategic Planning Process, Journal of Small Business Management, Vol. 17, No. 3, juillet 1979, pp.19-27. 
Robinson, Richard B. Jr. et Littlejohn, William F., Important contingencies in small firmplanning, Journal of Small Business management, Juillet $1981, \mathrm{p} .45$.

Robinson, Richard B. Jr., Logan, John E. et Salem Moragea Y., Strategic Versus Operational Planning In Small Retail Firms, American Journal of Small Business, 1986, p. 7.

Robinson, Richard B. Jr. et Pearce II, John A., Research Thrusts in Small Firm Strategic Planning, Academy of Management Review, 1984, Vol. 9, No. 1, p. 128.

Robinson, Richard B., Pearce II, John A., Vozikis, George S. et Mescon, Timothy $S$., The relationship between stage of development and small firm planning and performance, Journal of Small Business Management, Avril 1984, p.45.

Rue, Leslie W. et Holland, Phyllis G., Strategic Management, concepts and experiences, McGraw-Hill Book Company, Montréal, 1986,883 pages.

Schollamer, $H$. et Kuriloff, A., Entrepreneurship and Small Business Management, New York, John Wiley and Sons, 1979.

Sexton, Donald L. et Van Auken, Philip, Prévalence of strategic planning in small business, Journal of Small Business Management, Juillet 1982, p.21. 
Shrader, Charles B., Mulford, Charles L, et Blackturn, Virginia L., Strategic and operational planning, uncertainty, and performance in small firms, Journal of Small Business Management, Vol. 27, No. 4, octobre 1989, p.45.

Shuman, Jeffrey C. et Seeger, John A., The theory and practice of strategic management in smaller rapid growth firms, American Journal of Small Business, Vol, 18, No. 6, 1986, p. 48 .

Siegel, Eric, Schultz, Loren, Ford, Brian et Carney, David, The Arthur Young business plan guide, New York, John Wiley and Sons, 1987 .

Sonfielk, C., The Contingency Plan - How to Profit From Opportunities Created by Others, American Journal of Smal Business, Vol. IX, No. 1, été 1984, pp.44-48.

Stoner, Charles R., Planning in small manufacturing firms: a survey, Journal of Small Business Management, Janvier 1983, p. 34.

Timmons, Jeffrey, Smollen, Leonard E. et Dingee, Al exander M., New venture creation, a guide to entrepreneurship, second edition, Homewood, III., Richard D. Irwin, 1985.

Thurston, Philip H., La planification dans les PME, Harvard L'Expansion, Printemps 1984, p.24.

Unni V. K., DBA, Associate Professor of Management, Illinois State University, The Role of Strategic Planning in Small Businesses, Long Rang Planning, Vol. 14, Avril 1981, p.54. 
Van Kirk, John E. et Noonan, Kathleen, Key factors in strategic planning, Small Business Institute Directors Association, July 1982, p. 1 .

Venkatraman, N., Strategic orientation of business enterprises: the construct, dimensional ity and measurement, Management Science, vol. 35, No. 8, August 1989, p.942.

Vozikis, G.S. et Glueck, W.F., Small Business Problems and Stages of Development, Academy of Management Proceedings, 1980.

Wheelen, Thomas L. et Hunger, David J., Strategic Management, Addison-Wesley publishing Company, New York, 1990, 406 pages.

Wyant, R., The business failur record, New York: Dun and Bradstreet, 1977 . 
ANNEXE 1

QUEST IONNA IRE 
QUEST IONNA IRE

LA PHASE DE PRE-DEMARRAGE:

CAS D'ENTREPRISES DE SERVICES.

Caroline Huot, etudiante PME, U.R.A.C.

MAI 1992 


\section{INFORMATIONS GENERALES}

Le questionnaire qui suit s'inscrit dans le cadre d'une recherche sur les entrepreneurs qui viennent de démarrer leur entreprise ainsi que sur les pratiques de gestion. 11 a pour objectif de recueillir certaines informations sur vos caractéristiques personnelles comme dirigeant de PME et sur votre facon de gerer votre entreprise. Vous comprenez qu'il est important pour nous et pour vous-meme de connaitre vos opinions sur ces sujets.

Vous pouvez etre assure que vos reponses seront traitees confidentiellement et que vos resultats serviront exclusivement à des fins de recherche. Vous pouvez donc répondre en toute confiance et en toute sincérité. Nous aimerions connaitre ce que vous pensez réellement et non pas ce que vous croyez etre la bonne reponse. Malgré la longueur apparente de ce questionnaire, celui-ci se complète facilement puisque les questions ne demandent pas de réponses à développement.

Nous aimerions aussi insister sur quelques points pour vous aider a repondre au questionnaire.

1) Le genre de questions que nous vous posons varie d'une partie à l'autre du questionnaire de meme que les reponses possibles. Avant chaque partie du questionnaire, ecoutez bien nos directives. Si nos explications ne vous apparaissent pas claires, veuillez s.v.p. nous le faire savoir.

2) Répondez spontanément aux questions.

3) Il n'y a pas de bonnes ou de mauvaises réponses.

4) C'est votre opinion personnelle qui compte. Répondez avec franchise et honneteté.

5) Si vous faites une erreur, prenez soin de nous aviser.

La forme masculine est d'usage dans le questionnaire pour faciliter la lecture. Sauf lorsque le contexte l'indique autrement, la plupart des observations concernent autant les hommes que les femmes entrepreneurs.

\section{Merci pour votre précieuse collaboration.}

Caroline Huot, etudiante PMO, U.Q.A.C. 
SECTION A

RENSE IGNEMENTS SUR L'ENTREPRISE 


\section{A.1 Renseignements sur l'entreprise.}

Dans cette section, nous desirons recueillir des informations sur votre entreprise.

Nom de l'entreprise:

Localisation de l'entreprise (Région-Ville):

Date d'enregistrement/incorporation: Mois

Annee

Début officiel des operations:

Mois

Année

Secteur d'activités:

Forme légale de l'entreprise: 1) Proprietaire unique

2) Societé avec associés

3) Compagnie par actions

4) Cooperative

5) Société en commandite 
Dans votre entreprise, combien $y a-t-11$ de perzonnes ayant des llene familiaux avec vous?

(ex: belle falille, père, enfants, conjoint oncle, tante)

Qui est le principal responsable des activites quotidiennes de l'entreprise?
1) Vous
2) Partenaire
3) Administrateur
4) Autres (Précisez)

Quel est le principal responsable des activités suivantes:

$$
\text { Vous Autres (précisez) }
$$

Opérations

Marketing

Ressources hunaines

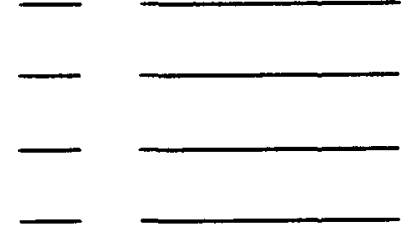


Quel est le nombre d'employes (en moyennel dans votre entreprise? (incluant le dirigeant)

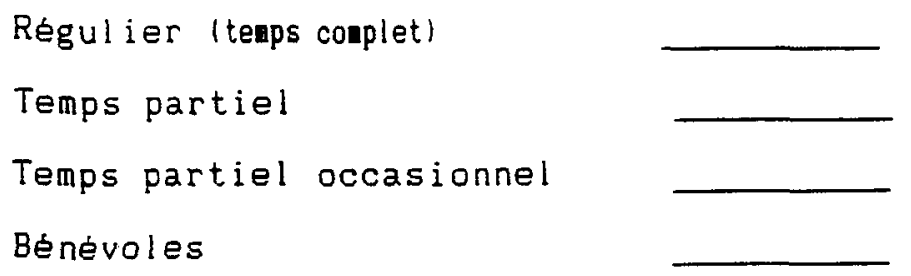

Quel est le nombre d'employes par catégorie d'emploi:

Cadre

Personnel de bureau

Personnel de vente

Personnel executant

Autres

Quel est le nombre d'heures que vous consacrez en moyenne par semaine aux affaires de l'entreprise: Hres

Actuellement, votre entreprise enregistre: 1) Des profits

2) Des pertes

3) $\mathrm{Ni}$ profit, ni perte 


\section{A.2 Motivations de démarrage du répondant}

Quelles ont éte les principales raisons qui vous ont motivé à fonder votre entreprise? (indiquer les numeros correspondant à vos trois preaiers choix)

1) Désir d'indépendance

2) Occuper mes temps libres

3) Me valoriser personnellement

4) Etre autonome financièrement $1^{\circ}$ choix:

5) Relever un défi

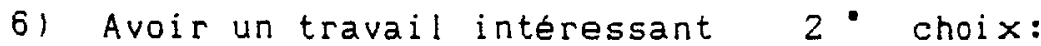

7) Faire de l'argent

8) Etre mon propre patron

3. choix:

9) Créer mon emploi

10) Réaliser un rêve

11) Créer une entreprise à succés

12) Autres 
Quel est le principal facteur ou événement qui vous a incité à lancer votre entreprise?

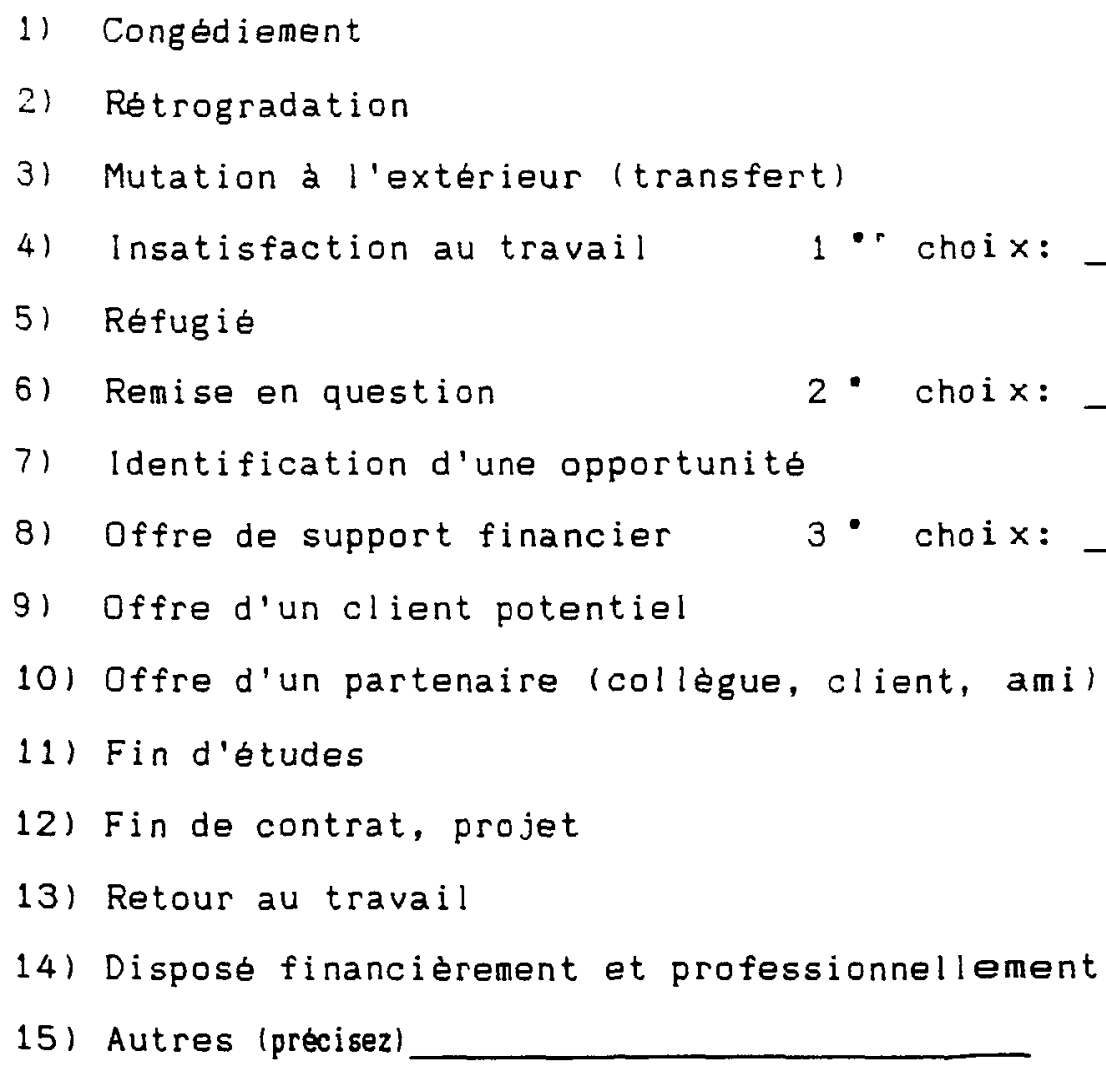

1) Congediement

2) Rétrogradation

3) Mutation à l'extérieur (transfert)

4) Insatisfaction au travail

1 -r choix:

5) Réfugié

6) Remise en question

2. choix:

7) Identification d'une opportunite

8) Offre de support financier

3. choix:

9) Offre d'un client potentiel

10) Offre d'un partenaire (collegue, client, ami)

11) Fin d'études

12) Fin de contrat, projet

13) Retour au travail

14) Disposé financièrement et professionnellement

15) Autres (precisez)

L'idée d'affaires:

Comment avez-vous trouve votre idee d'affaires? (encerclez votre choix)
A) De la PME où je travaillais
B) De la grande entreprise où je travaillais
C) Un passe-temps (loisirs)
D) Un deuxième emploi
E) Association avec inventeur
F) Une innovation
G) Une recherche active d'opportunités
H) Une demande spécifique d'un client
F) Autres (précisez) 
158

SECTION B

LES COMPORTEMENTS LORS DE LA PHASE DE PREDEMARRAGE 
B. Les comportements lors de la phase de prédemarrage

Cette section du questionnaire vise a recueillir des informations sur la phase de prédémarrage. Cette phase correspond à la période précédant le début officiel des operations de l'entreprise.

Combien de mois avez-vous consacré à la préparation de votre projet d'entreprise? mois

Lors de la phase de predemarrage, avez-vous formulé un plan d'affaires?
1) oui
2) non

Avez-vous bénéficié d'une formation vous permettant de mieux structurer $\begin{array}{lll}\text { votre projet? } & \text { 1) oui } & \text { 2) non }\end{array}$

Si oui, quel est son nom?

\section{B.1 Mission et objectifs}

1) Dans quelle mesure aviez-vous précisé chacun des él ements suivants lors de la phase de prédémarrage ? lencerclez le chiffre correspondant à votre choix)

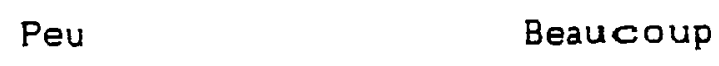

Le service à offrir

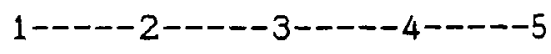

Le territoire vise

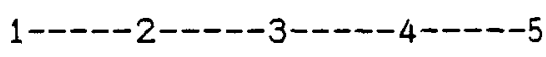

La clientele visee

$1-----2-----3-----4-----5$

Les modes d'operations

$1-----2-----3-----4-----5$

(ex: organisation du travail, equipenent) 
2) Aviez-vous fixé des objectifs?

1) oui

2) non

lex: chiffres d'affaires, profits, création d'eaploil

3) Quels etaient les principaux objectifs poursuivis par cette future entreprise?

4) Quelle était la periode couverte par ces objectifs?
A) moins d'un an
B) 1 a 2 ans
C) 3 à 5 ans
D) Non précisee 


\section{B.2 Connaissances du secteur d'activités et de la gestion}

A noter que nous desirons savoir quelles etaient vos connaissances en rapport à ce secteur d'activités et à la gestion avant de partir en affaires

Pas du tout Un peu Moyennement Beaucoup Tout à fait

Avant de prendre la decision finale de partir en affaires dans ce secteur d'activités: Idans l'espace réservé, inscrivez le chiffre correspondant à votre niveau de connaissance)

1) Je connaissais la tendance du marché.

2) Je connaissais le volume potentiel de ventes de la future entreprise.

3) Je connaissais les besoins en main-d'oeuvre afin d'operer une telle entreprise.

4) Je connaissais le volume minimale de ventes necessaire pour rencontrer les dépenses d'opérations (point mort).

5) Je connaissais bien le montant d'investissement requis afin de creer l'entreprise.

6) Je connaissais les principaux concurrents de ce secteur.

7) Je connaissais déjà des clients intéressés par mes produits.

8) Je connaissais la somme de travail que représentait la creation et la gestion d'une entreprise.

9) Je connaissais l'importance et le rale de l'innovation dans ce secteur d'activités.

10) Je connaissais les principaux fournisseurs de ce secteur.

11) Je connaissais les principales sources de financement permettant de créer l'entreprise. 


\section{B.3 ANALYSE ET DIAGNOSTIC}

\section{B.3.1 Environnement général}

Lors de la phase de predemarrage, dans quelle mesure aviez-vous analyse chacun des facteurs suivants? (encerclez le chiffre correspondant à votre choix)

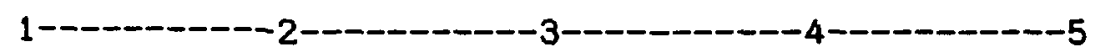

$\begin{array}{ccccc}\text { Pas du Un peu } & \text { Moyen- } & \text { Beaucoup } & \text { Tout a } & \text { Ne s'applique } \\ \text { tout } & & \text { nement } & \text { fait } & \text { pas }\end{array}$

Conjoncture économique (croissance, récession)

Taux de chómage

$\begin{array}{llllll}1 & 2 & 3 & 4 & 5 & 9\end{array}$

Taux d'interet

Taux d'inflation

Valeur du dollar canadien

Normes environnementales

Temperature et saison

Tendances démographiques

Technologies disponibles

Subventions gouvernementales (ex:argent)

Aides gouvernementaleslex: conseils professionnels)

$\begin{array}{llllll}1 & 2 & 3 & 4 & 5 & 9\end{array}$

Lois et réglementations:

CSST

Municipal

$B$ atiment

Santé et hygiene

Normes du travail

Fiscal i té (taxes/iupôts) $\begin{array}{llllll}1 & 2 & 3 & 4 & 5 & 9\end{array}$

123454

$12345 \quad 9$

$\begin{array}{llllll}1 & 2 & 3 & 4 & 5 & 9\end{array}$

$\begin{array}{llllll}1 & 2 & 3 & 4 & 5 & 9\end{array}$

$\begin{array}{llllll}1 & 2 & 3 & 4 & 5 & 9\end{array}$

$\begin{array}{llllll}1 & 2 & 3 & 4 & 5 & 9\end{array}$

$\begin{array}{llllll}1 & 2 & 3 & 4 & 5 & 9\end{array}$

$\begin{array}{llllll}1 & 2 & 3 & 4 & 5 & 9\end{array}$

$\begin{array}{llllll}1 & 2 & 3 & 4 & 5 & 9 \\ 1 & 2 & 3 & 4 & 5 & 9 \\ 1 & 2 & 3 & 4 & 5 & 9 \\ 1 & 2 & 3 & 4 & 5 & 9 \\ 1 & 2 & 3 & 4 & 5 & 9 \\ 1 & 2 & 3 & 4 & 5 & 9 \\ 1 & 2 & 3 & 4 & 5 & 9\end{array}$

Autres: (précisez) 


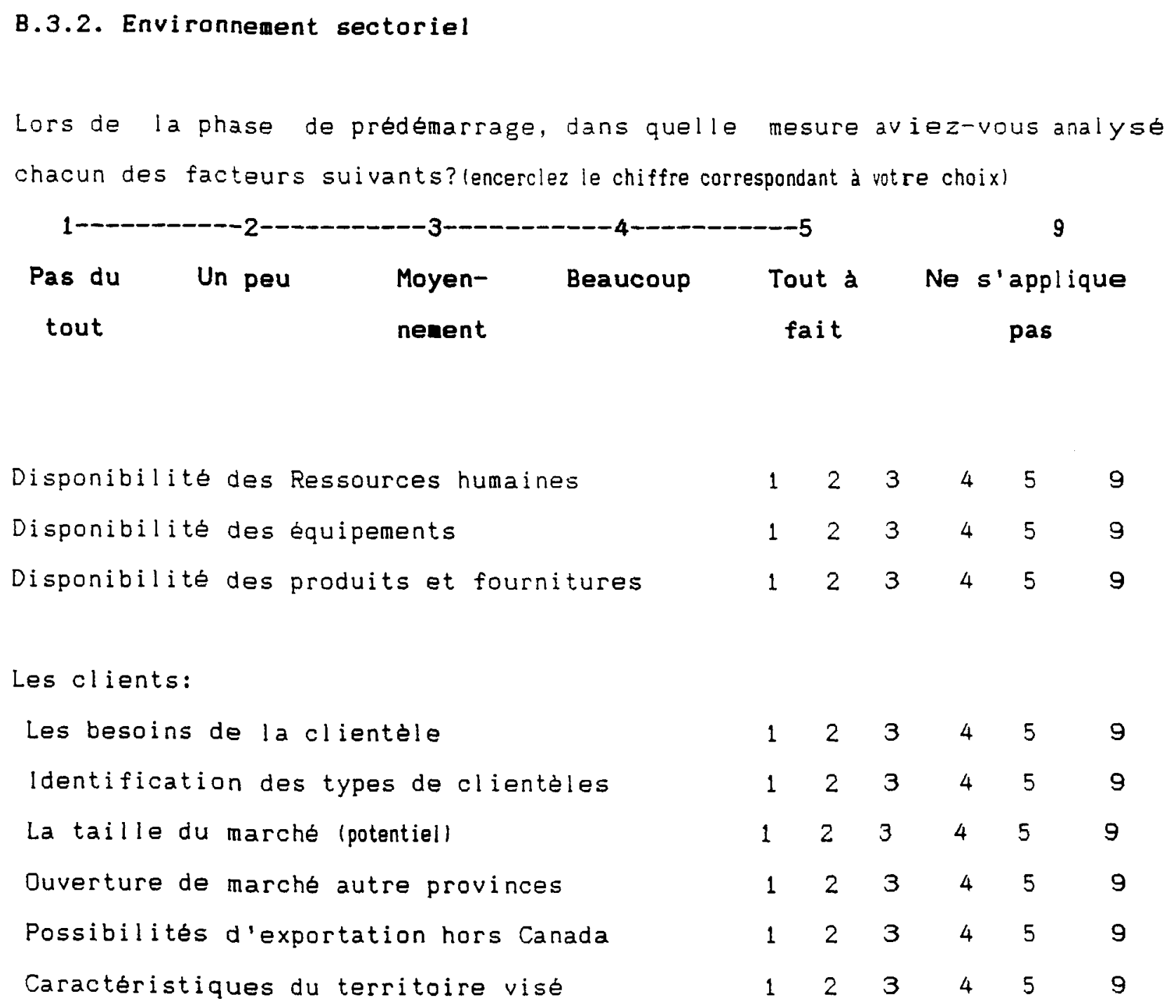




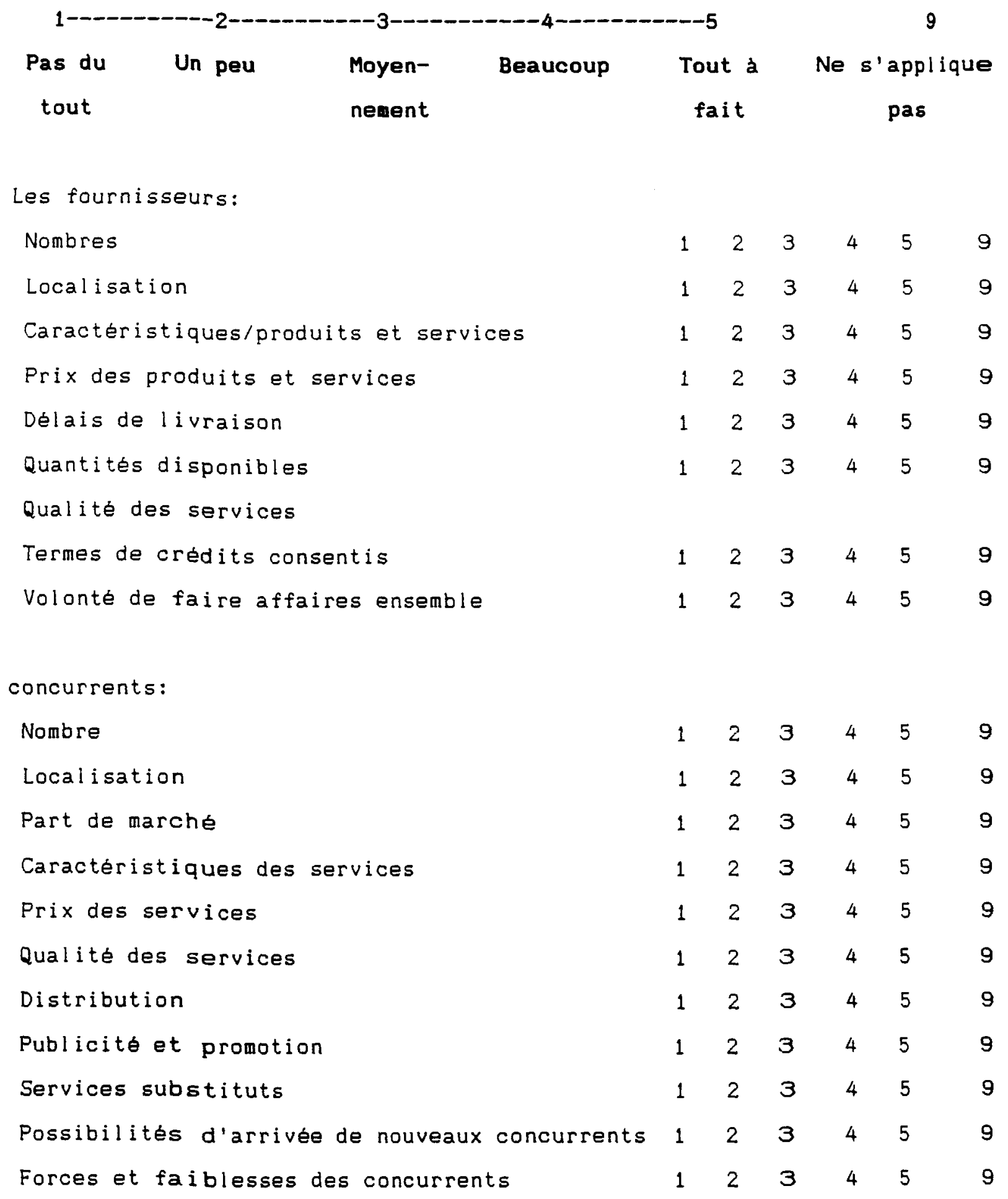




\section{B.3.3 Opportunités et menaces}

Lors de la phase de predemarrage, quels etaient les principaux facteurs de l'environnement qui favorisaient la creation/demarrage de la nouvel le entreprise? |référez vous au tableau A et inscrivez le chiffre correspondant à votre choix)

$$
\text { 1*r choix: } \quad 2^{*} \operatorname{choix}: \ldots
$$

Lors de la phase de prédemarrage, quels étaient les principaux facteurs de l'environnement qui menaçaient la création/démarrage de la nouvel le entreprise? (référez vous au tableau A et inscrivez le chiffre correspondant à votre choix)

$$
\text { 1*r choix: } \quad 2^{\bullet} \operatorname{choix}: \quad 3^{\bullet} \operatorname{choix} \text { _ }
$$

\section{Tableau A}

1) Conjoncture écononique

3) Taux d'interet

5) Valeur du dollar canadien

7) Tenperature et saison

91 Technologie disponible

11) Aide gouvernementale

Lois et róglementations:

12) CSST

14) Bătiment

16) Nornes du travail

18) Disponibilité ressources huaines

20) Disponibilite des approvisionnements

22) Identification des types de clienteles

24) Cycle de vie du secteur d'activités

261 Ouverture marchés étrangers(hors Québec) Les fournisseurs:

28) Nosbres

30) Caractéristiques des services

32) Délais de livraison

34) Qualité des services

Les concurrents:

36) Nonbre

38) Part de arche

40) Prix des services

42) Distribution

441 Concurrents indirects
2) Taux de chônage

4) Taux d'inflation

6) Normes environnenentales

8) Tendances dénographiques

10) Subventions gouvernementales

13) Municipal

15) Santé et hygiène

17) Revenu (taxes/inpôts)

19) Disponibilité ress. natérielles

21) Les besoins de la clientele

23) La taille du marché

25) Cycle de vie des services

27) Caractéristiques du territoire vise

29) Localisation

31) Prix des services

33) Quantités disponibles

35) Termes de credits consentis

37) Localisation

39) Caractéristique des services

41) Qualité des services

431 Publicité et pronotion

45) Arrive de nouveaux concurrents 


\section{B.3.4. Environnement interne}

Lors de la phase de prédemarrage, dans quelle mesure aviez-vous analyse chacun des facteurs suivants?

$\begin{array}{lcccc}\text { Pas du Un peu } & \text { Moyen- } & \text { Beaucoup } & \text { Tout a } & \text { Ne s'applique } \\ \text { tout } & & \text { nement } & \text { fait } & \text { pas }\end{array}$

Aspect marketing

Prix des services à offrir

$\begin{array}{llllll}1 & 2 & 3 & 4 & 5 & 9\end{array}$

Gamme de service à offrir

Qualite des services à offrir

$\begin{array}{llllll}1 & 2 & 3 & 4 & 5 & 9\end{array}$

Uniformisation des services

Capacite de production de services

$\begin{array}{llllll}1 & 2 & 3 & 4 & 5 & 9\end{array}$

Service à la clientele (garantie, installation)

$\begin{array}{llllll}1 & 2 & 3 & 4 & 5 & 9\end{array}$

Promotion et publicité

$\begin{array}{llllll}1 & 2 & 3 & 4 & 5 & 9\end{array}$

Sources d'information

Délais de livraison des services

$\begin{array}{llllll}1 & 2 & 3 & 4 & 5 & 9\end{array}$

Accessibilité aux services

Possibilite de développer nouveaux services

$\begin{array}{llllll}1 & 2 & 3 & 4 & 5 & 9\end{array}$

$\begin{array}{llllll}1 & 2 & 3 & 4 & 5 & 9\end{array}$

$123 \quad 4 \quad 5 \quad 9$

$123 \quad 4 \quad 5 \quad 9$

$1253 \quad 4 \quad 5 \quad 9$

\section{Aspects operationnels}

Besoins en equipement et fournitures

$\begin{array}{llllll}1 & 2 & 3 & 4 & 5 & 9\end{array}$

Besoins en ressources (électricités, gaz, eau)

Standardisation des méthodes de travail

Critere de selection des fournisseurs

Niveau de stock de fournitures

$\begin{array}{llllll}1 & 2 & 3 & 4 & 5 & 9\end{array}$

Coits d'opération de l'entreprise

Criteres de localisation de l'entreprise

$\begin{array}{llllll}1 & 2 & 3 & 4 & 5 & 9\end{array}$

$\begin{array}{llllll}1 & 2 & 3 & 4 & 5 & 9\end{array}$

$123 \quad 4 \quad 5 \quad 9$

$\begin{array}{llllll}1 & 2 & 3 & 4 & 5 & 9\end{array}$

$\begin{array}{llllll}1 & 2 & 3 & 4 & 5 & 9\end{array}$ 


$\begin{array}{lcccc}\text { Mas du Un peu } & \text { Moyen- } & \text { Beaucoup } & \text { Tout a } & \text { Ne s'applique } \\ \text { tout } & \text { nement } & \text { fait } & \text { pas }\end{array}$

$\begin{array}{lllllll}\text { Critéres de sélection des équipements } & 1 & 2 & 3 & 4 & 5 & 9 \\ \text { Aménagement physique de l'entreprise } & 1 & 2 & 3 & 4 & 5 & 9 \\ \text { Criteres de conträle de la qualité } & 1 & 2 & 3 & 4 & 5 & 9 \\ \text { Gestion des achats } & 1 & 2 & 3 & 4 & 5 & 9 \\ \text { Planification et contrále des opérations } & 1 & 2 & 3 & 4 & 5 & 9\end{array}$

\section{Aspects humains}

Besoins en ressources humaines

$\begin{array}{llllll}1 & 2 & 3 & 4 & 5 & 9\end{array}$

Táches à exécuter

Moyens de coordination des activites

Qualifications requises

Rémuneration (salaires et avantages sociaux)

Méthodes de recrutement (ex: Journaux)

Critère de selection (ex: années d'experience)

L'accueil du nouvel employé

Exigences de santé et securité au travail

Besoins de formation

$\begin{array}{llllll}1 & 2 & 3 & 4 & 5 & 9\end{array}$

$\begin{array}{llllll}1 & 2 & 3 & 4 & 5 & 9\end{array}$

$\begin{array}{llllll}1 & 2 & 3 & 4 & 5 & 9\end{array}$

1234549

$12345 \quad 5 \quad 9$

Philosophie de gestion (valeur, culture)

Méthodes d'évaluation du personnel

Création d'un conseil d'administration

$\begin{array}{llllll}1 & 2 & 3 & 4 & 5 & 9\end{array}$

$\begin{array}{llllll}1 & 2 & 3 & 4 & 5 & 9\end{array}$

$\begin{array}{llllll}1 & 2 & 3 & 4 & 5 & 9\end{array}$

$\begin{array}{llllll}1 & 2 & 3 & 4 & 5 & 9\end{array}$

Utilisation de conseillers externes

$\begin{array}{llllll}1 & 2 & 3 & 4 & 5 & 9\end{array}$

$\begin{array}{llllll}1 & 2 & 3 & 4 & 5 & 9\end{array}$

$\begin{array}{llllll}1 & 2 & 3 & 4 & 5 & 9\end{array}$

1234599 


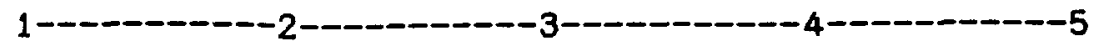

Pas du

Un peu

tout
Moyen-

nement
Beaucoup

Tout à

fait
9

Ne s'applique

pas

\section{Aspects financiers}

Besoins de fonds

Capacité d'endettement

$\begin{array}{llllll}1 & 2 & 3 & 4 & 5 & 9\end{array}$

Coiits globaux

Chiffre d'affaires potentiel

Point mort (seuil de rentabilité)

Pertes et/ou profits potentiels

Variation du fonds de roulement

Taxes et impòts à payer

Procedures de contrôle financiers

Budgets prévisionnels

Sources de financement

$\begin{array}{llllll}1 & 2 & 3 & 4 & 5 & 9\end{array}$

$\begin{array}{llllll}1 & 2 & 3 & 4 & 5 & 9\end{array}$

$\begin{array}{llllll}1 & 2 & 3 & 4 & 5 & 9\end{array}$

$123 \quad 4 \quad 5 \quad 9$

$\begin{array}{llllll}1 & 2 & 3 & 4 & 5 & 9\end{array}$

$\begin{array}{llllll}1 & 2 & 3 & 4 & 5 & 9\end{array}$

$\begin{array}{llllll}1 & 2 & 3 & 4 & 5 & 9\end{array}$

$\begin{array}{llllll}1 & 2 & 3 & 4 & 5 & 9\end{array}$

$\begin{array}{llllll}1 & 2 & 3 & 4 & 5 & 9\end{array}$

$\begin{array}{llllll}1 & 2 & 3 & 4 & 5 & 9\end{array}$

\section{Aspects informationnels}

Prix de revient (coût unitaire)

Systeme comptable approprié

Systeme informatique (équipenent, logiciel)

$\begin{array}{llllll}1 & 2 & 3 & 4 & 5 & 9 \\ 1 & 2 & 3 & 4 & 5 & 9 \\ 1 & 2 & 3 & 4 & 5 & 9\end{array}$

\section{Aspects légaux}

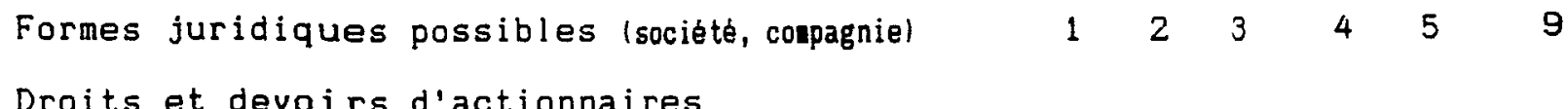
(convention entre actionnaire)

Ententes légales (ex: foralisation des contrats de ventes)

$\begin{array}{llllll}1 & 2 & 3 & 4 & 5 & 9\end{array}$

$\begin{array}{llllll}1 & 2 & 3 & 4 & 5 & 9\end{array}$




\section{B.3.5 Forces et Faiblesses}

Lors de la phase de prédemarrage, quelles étaient les principales forces qui semblait avantager la future entreprise?

Lors de la phase de prédémarrage, quelles etaient les principales faiblesses qui semblait desavantager la future entreprise ? 


\section{B.3.6 SOURCES D'INFORMATION}

Lors de l'analyse de l'environnement, vous avez utilisé différentes sources d'informations. En vous référant aux choix suivants, préciser les sources consultées et l'intérêt des informations obtenues par ces consultations.

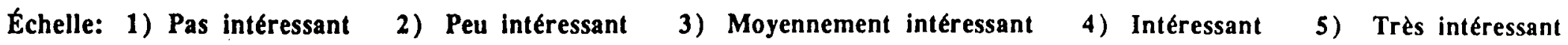

\section{SOURCES D'INFORMATION}

\begin{tabular}{|c|c|c|c|c|c|c|c|c|c|}
\hline \multirow[b]{2}{*}{$\begin{array}{l}\text { Information } \\
\text { sur }\end{array}$} & \multicolumn{4}{|c|}{ Sources impersonnelles } & \multicolumn{5}{|c|}{ Sources personnelles } \\
\hline & $\begin{array}{c}\text { Revues } \\
\text { Journaux }\end{array}$ & $\begin{array}{l}\text { Repertoires } \\
\text { Statistiques }\end{array}$ & $\begin{array}{l}\text { Documents } \\
\text { concurrence }\end{array}$ & $\begin{array}{l}\text { Bibliotheques } \\
\text { ministeres }\end{array}$ & A mis & Parents & Concurrence & Clients & $\mid \begin{array}{c}\text { Autres } \\
\text { (precisez) }\end{array}$ \\
\hline Sur l'Gconomie & & & & & & & & & \\
\hline Politiques gourvernementales & & & & & & & & & \\
\hline Aide \& support gouvernem. & & & & & & & & & \\
\hline Lois et règlements & & & & & & & & & \\
\hline Clientele & & & & & & & & & \\
\hline Foumisseurs & & & & & & & & & \\
\hline Matieres premitres & & & & & & & & & \\
\hline Machineric \& Gquipements & & & & & & & & & \\
\hline Sources de financement & & & & & & & & & \\
\hline Concurrents & & & & & & & & & \\
\hline Marches Etrangers & & & & & & & & & \\
\hline Autres informations (precisez) & & & & & & & & & \\
\hline
\end{tabular}




\section{B.3.7 Personnes ressources}

Lors de la phase de prédemarrage, quelles sont les personnes qui ont participé activement à l'élaboration de votre projet d'entreprise. (encerclez le chiffre correspondant à votre choix)

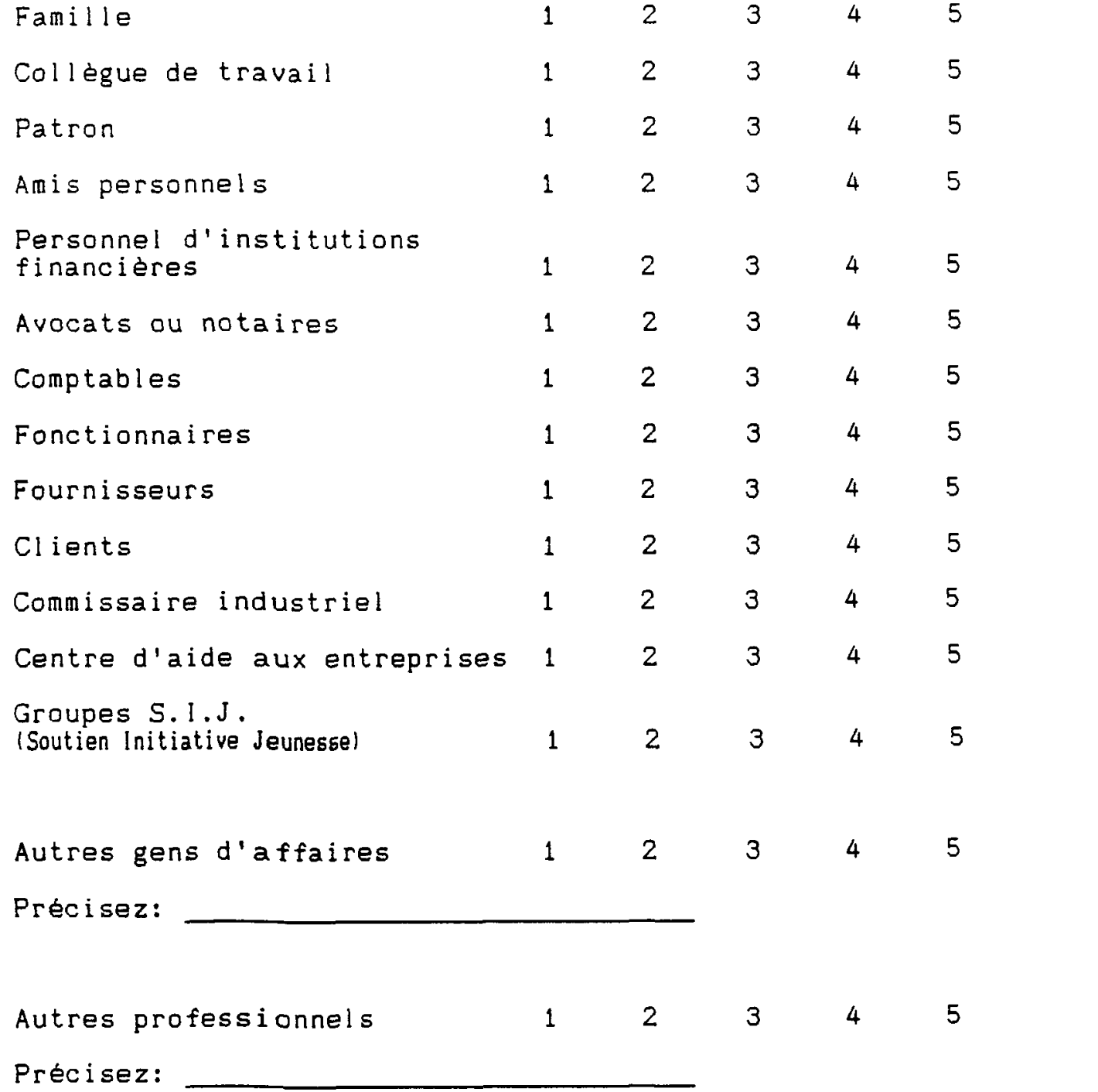

\section{Inactif}

\section{1}




\section{B.3.8 Personnes ressources}

Lors de la phase de prédemarrage, indiquez les raisons pour lesquelles vous avez contacté les organismes/personnes ressources suivants:

(encerclez la lettre correspondant à votre choix)

$\begin{array}{lllc}\text { Obtention de } & \text { Support et } & \text { Information ou } & \text { Ne s'applique } \\ \text { ressources } & \text { encouragenent } & \text { conseil sur les } & \text { pas } \\ \text { F) Financières } & & \text { affaires et/ou } & \\ \text { M) Matérielles } & & \text { le secteur } & \\ \text { H) Hunaines } & & \text { d'activites } & \end{array}$

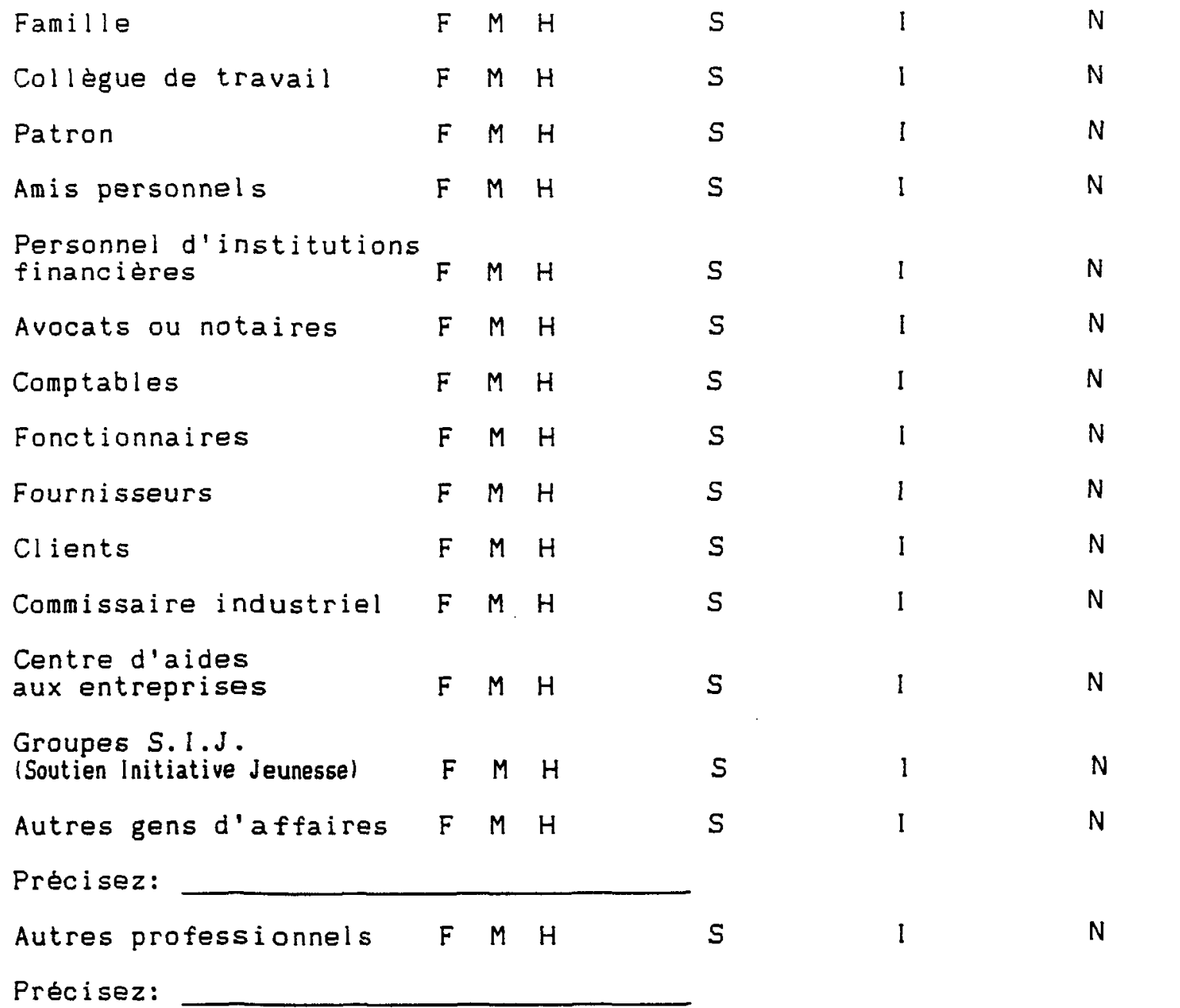




\section{B.3.9 Cholx stratégiques en phase de prédémarrage}

Lors de la phase de prédemarrage, qualles etaient les caractéristiques speclfiques de vos orientations ? (encerclez le chiffre correspondant à votre choix)

$\begin{array}{llllllll}\text { Marché } & \text { 1) Local 2l Régional } & \text { 3) Provincial } & 4 \text { National } & \text { 5) International } \\ \text { Marché } & \text { Peu concurrence } & 1 & 2 & 3 & 4 & 5 & \text { Beaucoup conc. } \\ \text { Marché } & \text { Nouveau } & 1 & 2 & 3 & 4 & 5 & \text { Existant } \\ \text { Image/clientèle } & \text { Populaire } & 1 & 2 & 3 & 4 & 5 & \text { Prestige } \\ \text { Clientele } & \text { Masse } & 1 & 2 & 3 & 4 & 5 & \text { Ciblé } \\ \text { Technologie } & \text { Peu sophistiqué } & 1 & 2 & 3 & 4 & 5 & \text { Trés sophist. } \\ \text { Nature du service } & \text { Spécialiste } & 1 & 2 & 3 & 4 & 5 & \text { Généraliste } \\ \text { Service } & \text { Traditionnel } & 1 & 2 & 3 & 4 & 5 & \text { Innovateur } \\ \text { Gamme de service } & \text { Peu étendue } & 1 & 2 & 3 & 4 & 5 & \text { Très étendue } \\ \text { Qualité service } & \text { Inférieure } & 1 & 2 & 3 & 4 & 5 & \text { Supérieure } \\ \text { Prix } & \text { Faible } & 1 & 2 & 3 & 4 & 5 & \text { Elevé } \\ \text { Distribution } & \text { Exclusive } & 1 & 2 & 3 & 4 & 5 & \text { Intensive } \\ \text { Promotion } & \text { Faible } & 1 & 2 & 3 & 4 & 5 & \text { Elevée }\end{array}$


SECTION C

LES CARACTERISTIQUES PERSONNELLES

DE L'ENTREPRENEUR 
C. Les caractéristiques personnelles de l'entrepreneur

Cette derniere section vise à recueillir des informations concernant vos caractéristiques personnelles. (nous vous assurons de la confidential ité de vos reponses).

Age du répondant:

Sexe du répondant: 1 ) Féminin 2) Masculin

Etat civil: 1) Marié ou union libre

2) Célibataire, séparé, divorcé, veuf

Nombre d'année de scolarite:

Dernier niveau de scolarité: 1) Primaire

2) Secondaire général

3) Secondaire professionnel

4) Collégial general

5) Collégial professionnel

6) Universitaire 
Votre formation scolaire vous a-t-elle permis d'acquerir des connaissances et/ou de développer des habiletés en relation avec votre entreprise? (encerclez votre/vos choix)
A) Connaissances en gestion
B) Connaissances techniques/spécialisees
C) Connaissances du secteur d'activités
D) Connaissances en affaires
E) Connaissances en démarrage d'entreprise
F) Autres

Emploi antérieur: Taille de l'entreprise 11 Moins de 10 employes

2) 11 à 50 employés

3) 51 à 250 employés

4) 251 à 500 emp 1 oyés

5) Plus de 500 employés

Avez-vous déjà été propriétaire/partenaire dans une autre entreprise?

1) oui 2) non

Avez-vous déjà fait faillite? 11 oui 21 non

Vos emplois antérieurs vous ont-ils permis d'acquérir une experience de travail pertinente en relation avec vos taches actuelles?
A) Expérience de gestion
B) Expérience 1 iee au secteur d'activités
C) Les reponses $A$ et $B$
D) Autres 
Nombre de mois d'experience de travail avant de creer votre entreprise:

Avez-vous des personnes dans votre famille immediatequi sont en affaires? (conjoint, père, à̀re, frère, soeur, enfants)
1) oui
2) non

Si oui

secteur d'activitéde

votre entreprise?

1) oui

2) non 
NOM ET ADRESSE PERSONNELLE DU REPONDANT

Nom et prénom:

Adresse:

Code postal:

Tél ephone:

\section{NOM ET ADRESSE DE L'ENTREPRISE}

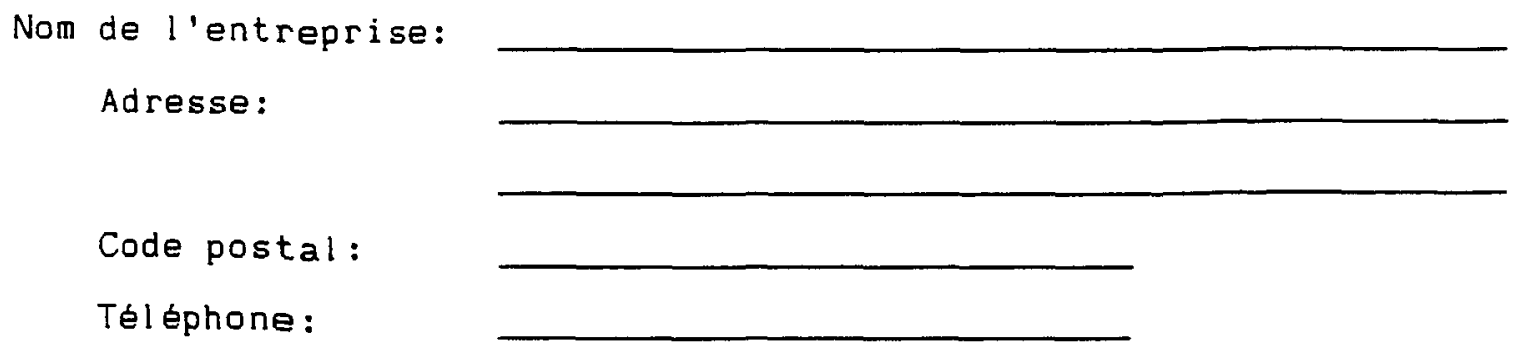

MERCI DE VOTRE COLLABORATION 
ANNEXE 2

COMPARAISON DES PLANS D'AFFAIRES 
ANNEX: II

TAIILAU COMPARATIF DU CONTENU IRS PIANS D'AFARRS

\begin{tabular}{|c|c|c|c|c|}
\hline TIMMONS el AL. (1985) & MANCUSO (1985) & McCLAUGiILIN (1985) & FORTIN (1986) & SIE(jEL eI AL. (1987) \\
\hline $\begin{array}{l}\text { 1. SOMMAIRE DU PLAN } \\
\text { - Fuits saillants }\end{array}$ & $\begin{array}{l}\text { SOMMAIRE DUPLAN } \\
-\quad \text { Fails saillants }\end{array}$ & $\begin{array}{l}\text { SOMMAIRE DE L'OPPORTUNITÉ } \\
\text { - Fails saillants }\end{array}$ & $\begin{array}{l}\text { SOMMAIRE DU PLAN } \\
- \text { Fails ssillants }\end{array}$ & $\begin{array}{l}\text { SOMMAIRE DU PLAN } \\
\text { - Fails saillants }\end{array}$ \\
\hline $\begin{array}{l}\text { 2. INDUSTRIE. ORGANISATION } \\
\text { ERODUITS SERYICES }\end{array}$ & & & & \\
\hline $\begin{array}{l}2.1 \text { Descriplion de lindustrie } \\
\text { Nouveaux produits, clients, } \\
\text { concurrents, presents el futurs, } \\
\text { envitonnement economique et } \\
\text { national }\end{array}$ & $\begin{array}{l}\text { Descriplion de l'industrie } \\
\text { Statistiques }\end{array}$ & Industrie: taille et croissance & $\begin{array}{l}\text { Indusirie: situalion acluelle et projetee, } \\
\text { opportunites et menaces, environne- } \\
\text { ment tconomique et national }\end{array}$ &.- \\
\hline 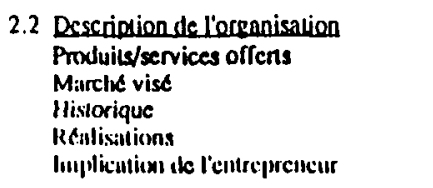 & $\begin{array}{l}\text { Description de ce qui caractérise } \\
\text { Lorgnnisnlion compare d la concumence } \\
\text { historique cl rénlisations }\end{array}$ & 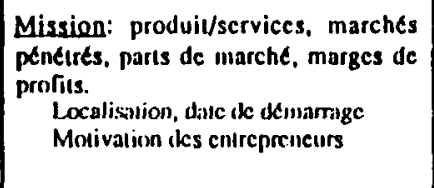 & $\begin{array}{l}\text { Description de l'enleserise el l'oppor- } \\
\text { unile } \\
\text { - marches vises } \\
\text { - hisiosique } \\
\text { - implication de l'cmireprencur }\end{array}$ & $\begin{array}{l}\text { Descrintion de l'entreprise } \\
-\quad \text { Marchds visds } \\
\text { - Basc geographique des marchis vises }\end{array}$ \\
\hline 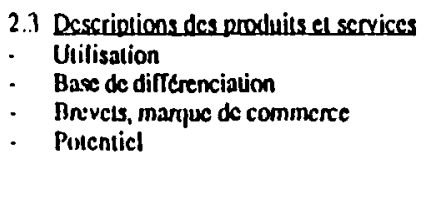 & 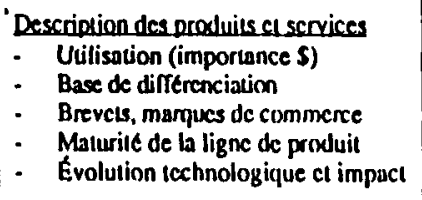 & $\begin{array}{l}\text { Descriplion produils el services } \\
- \text { Description vulgarisce } \\
\text { - Specifications succintes el chaires }\end{array}$ & 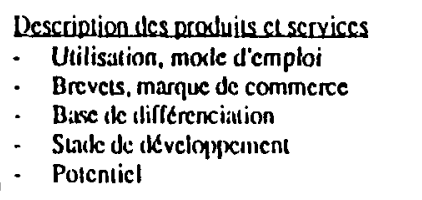 & $\begin{array}{l}\text { Descriplion des pooduits el services } \\
\text { Description physique } \\
\text { - Utilisation } \\
\text { - Stusle de developpement de produil }\end{array}$ \\
\hline $\begin{array}{l}2.4 \text { Sinudgic denule elude croissince } \\
\text { Description } \\
\text { Oljectifs vists }\end{array}$ & - & - & - & - \\
\hline
\end{tabular}

Source: Belley, Dussault et Lorrain, 1989 
ANNEXE ॥

TABLEAU CORPORATIF DU CONTENU DES PLANS D'AFFAIRES

\begin{tabular}{|c|c|c|c|c|}
\hline IROOKS \& STEVENS (1987) & DELL'ANIELLO (1987) & GASSE, et AL. (1988) & $(; 000(1989)$ & ONUDI (1978) \\
\hline $\begin{array}{l}\text { SOMMAIRE DU PLAN } \\
\cdot \quad \text { Fails saillants }\end{array}$ & $\begin{array}{l}\text { SOMMAIRE DU PLAN } \\
\text { - Faits soillants }\end{array}$ & $\begin{array}{l}\text { SOMMAIRE DU PLAN } \\
\text { - Fails saillanis }\end{array}$ & $\begin{array}{l}\text { SOMMAIRE DU PLAN } \\
\cdot \quad \text { Fails saillants }\end{array}$ & N/A \\
\hline \multicolumn{5}{|l|}{$\begin{array}{l}\text { INIDUSTRE, OROANISATION } \\
\text { PRODUITS/SERVICES }\end{array}$} \\
\hline & $\begin{array}{l}\text { Inchustrical cnvironncment } \\
\text { (voir section specifique) } \\
\text { Descripuion de lidec oui_doit } \\
\text { correspondre d la mission de } \\
\text { l'organisation. Identificalion produit }\end{array}$ & $\begin{array}{l}\text { Industric, secicur d'aclivile, Iendance, } \\
\text { contexte }\end{array}$ & 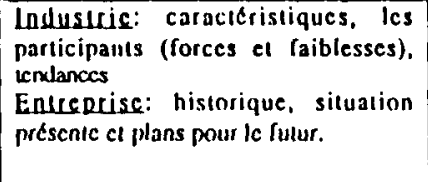 & $\begin{array}{l}\text { Industric: environnement ceonomique } \\
\text { industrict. financicr, social et autres } \\
\text { luke du proicl: capacite el emplacement de } \\
\text { l'usine } \\
\text { Bise gengroplyique de marche } \\
\text { Historique du profil. }\end{array}$ \\
\hline 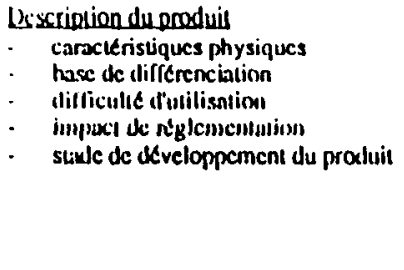 & 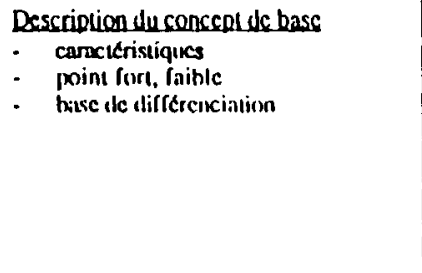 & 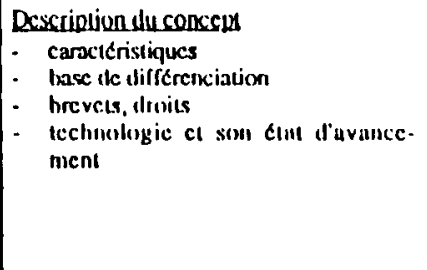 & 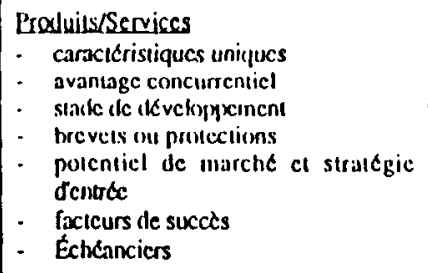 & Descriplion des produils el sous provluils \\
\hline
\end{tabular}




\begin{tabular}{|c|c|c|c|c|}
\hline TIMMONS el AL. (1985) suile & MANCUSO (1985) suile & MCCL.AU(BIILIN (1985) suife & FORTIN (1986) suile & SIEGEL el AL. (1987) suile \\
\hline 3. ANALYSE DU MARCHÉ & & & & \\
\hline $\begin{aligned} 3.1 \text { clicnls } & \text { - identificalion } \\
& \text { - segmentation } \\
& \text { - compontement d'achat }\end{aligned}$ & $\begin{array}{l}\text { Clicals - où le prosluit est vendu } \\
\text { - utilisateur final } \\
\text { - motivations d'achat } \\
\text { - principaux clients }\end{array}$ & $\begin{aligned} \text { Clicnls } & \text { - identificition } \\
& \text { - comportement d'achat }\end{aligned}$ & $\begin{aligned} & \text { Clicnis } \text { - identification } \\
& \text { - segmentition } \\
& \text { - comportenient d'achat }\end{aligned}$ & $\begin{array}{c}\text { Clicnls - marche primaire el secondaire } \\
\text { - scgmentation el importance }\end{array}$ \\
\hline $\begin{array}{l}3.2 \text { Marche: Tailles } \\
\text { Facteurs decerminants de la } \\
\text { demande } \\
\text { Croissance } \\
\text { Projections }\end{array}$ & $\begin{array}{l}\text { Marchs: historique } \\
\text { taille } \\
\text { tendances } \\
\text { positionnement sur le marche } \\
\text { localisation courbe de produit } \\
\text { projeclions } \\
\text { reglementations } \\
\text { saisonnicr }\end{array}$ & $\begin{array}{l}\text { Marchs: geographic } \\
\text { taille du marche } \\
\text { décrminants de la itemande } \\
\text { prix pret i payer } \\
\text { marche bien desservi ou non }\end{array}$ & $\begin{array}{l}\text { Manche: Lille } \\
\text { base geographique } \\
\text { determinarl itc la demande } \\
\text { croissance }\end{array}$ & $\begin{array}{l}\text { Marche: Deseription de l'industrie } \\
\text { Determinanls de la demande } \\
\text { Croissarice } \\
\text { Volatilite du marche } \\
\text { Reglementation }\end{array}$ \\
\hline $\begin{aligned} 3.1 \text { Concumence: } & \text { - Identification } \\
& \text { - Comparaison (4P) } \\
& \text { - Forces el faiblesses }\end{aligned}$ & $\begin{array}{ll}\text { Concuncencs: } & \text { - Identification } \\
& \text { - Nature de la concur- } \\
\text { rence } \\
\text { - Entrec de nouveaux } \\
\text { concurrents } \\
\text { - Forces ct faiblesses }\end{array}$ & 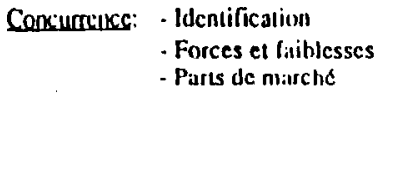 & 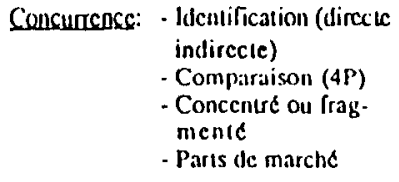 & $\begin{array}{ll}\text { Concuncenc: } & \text { - Identificalion } \\
& \text { - Forces cl faiblesses } \\
& \text { - Anticiper les nouveaux } \\
& \text { concurrents, les imita- } \\
& \text { teurs }\end{array}$ \\
\hline $\begin{array}{l}\text { 3.1 Parlde manchele chiffre diaffaires } \\
\text { - Base de penceration } \\
\text { - Proprieles uniques du produit }\end{array}$ & $\begin{array}{l}\text { Parde marche } \\
\text { Basc de penctration } \\
\text { Avantages concurtenticls }\end{array}$ & $\begin{array}{l}\text { Parn de manche et chiffere diffaircs } \\
\text { Avantages concurrentiels }\end{array}$ & Ban de marche ce chillics d'alfaires & $\begin{array}{l}\text { Pan de marche crechifles d'affaires } \\
\text { Presenter les cludes de marche faites. }\end{array}$ \\
\hline
\end{tabular}




\begin{tabular}{|c|c|c|c|c|}
\hline $\begin{array}{l}\text { IROOKS \& STEVENS (1987) } \\
\text { suile }\end{array}$ & DELL'ANIFILLO (1987) suile & (EASSE el Al.. (198X) suite & (:00) (1989) suife & ONUDI (197k) suile \\
\hline \multicolumn{5}{|l|}{ 3. ANALYSE DE MARCHE } \\
\hline $\begin{aligned} \text { Clicnts: } & \text { - identification et caractéristi- } \\
& \text { ques } \\
& \text { segmentation }\end{aligned}$ & $\begin{array}{l}\text { Clients: Identification } \\
\text { Segmentation } \\
\text { Segments non exploites } \\
\text { Comportements d'achat, pri- } \\
\text { maires, secondaires }\end{array}$ & $\begin{array}{l}\text { Clicnls: Calégories } \\
\text { Segmentation }\end{array}$ & $\begin{array}{ll}\text { Clienls: } & \text { Primaires - Type - } \\
& \text { Segmentation } \\
& \text { Profil } \\
& \text { Comportement d'achat }\end{array}$ & $\begin{array}{l}\text { Clients: Iuenúfication } \\
\text { Segmentation }\end{array}$ \\
\hline $\begin{array}{l}\text { Murchs: Base glographique } \\
\text { Demande }\end{array}$ & $\begin{array}{l}\text { Manchs: Taille } \\
\text { Tendances } \\
\text { Cruissance } \\
\text { Type (consommateur, indus- } \\
\text { tricl, revendeurs, gouverme- } \\
\text { mental) }\end{array}$ & $\begin{array}{l}\text { Mirche: Territoire } \\
\text { Taille } \\
\text { Caracteristiques } \\
\text { Terulances }\end{array}$ & $\begin{array}{l}\text { Marclk: Taille } \\
\text { Determistants de la demande } \\
\text { Saisonnier }\end{array}$ & $\begin{array}{l}\text { Marche: Taille } \\
\text { DCleminanls de la demande } \\
\text { Croissance } \\
\text { Projections }\end{array}$ \\
\hline $\begin{array}{l}\text { Concuricnce: Identification } \\
\text { Forces et faiblesses }\end{array}$ & $\begin{array}{ll}\text { Concurrence: } & \text { Identification } \\
& \text { Forces et faiblesses } \\
& \text { Localisation } \\
\text { Accroissement ou dimi- } & \text { nution } \\
\text { Concentre ou fragmente }\end{array}$ & $\begin{array}{ll}\text { Concurmence: } & \text { Identification } \\
& \text { Territoire } \\
& \text { Forces cl faillesses } \\
& \text { Augmentation ou diuni- } \\
\text { nution }\end{array}$ & $\begin{array}{l}\text { Concunence: Identification } \\
\text { Fores el faiblesses } \\
\text { Anuiciper le lutur }\end{array}$ & Concurrense: Idenuification \\
\hline 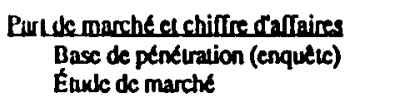 & & $\begin{array}{l}\text { Parde marche el chiffre d'sfaires } \\
\text { Avantoges concurrentiels }\end{array}$ & $\begin{array}{l}\text { Pande marche ef chiffre d'affaires } \\
\text { (enquetes, etuudes de marehe) }\end{array}$ & $\begin{array}{l}\text { Pande marche a chiffre d'affaices } \\
\text { Taux de ptictuation }\end{array}$ \\
\hline
\end{tabular}




\begin{tabular}{|c|c|c|c|c|}
\hline TIMAIONS el AL. (1985) sulie & MANCUSO (1985) suite & MrCl.AU(iIll.IN (1985) suite & FORTIN (19K6) suite & SIE(BIEL el AL. (1987) suile \\
\hline $\begin{array}{ll} & \text { Clicns dejà engages } \\
\text { Deicrmination de la pan de marche } & \text { Deicrmination des ventes }\end{array}$ & $\begin{array}{l}\text { Commandes fermes } \\
\text { - Deierminacion de la parl de marche }\end{array}$ & Décrmination de la parn de marché & $\begin{array}{l}\text { Délemination de la parl de marché } \\
\text { Déiermination des ventes }\end{array}$ & $\begin{array}{l}\text { Leltres d'intervention, arlicles } \\
\text { Analyscs industrielles } \\
\text { Determination des parts de marche } \\
\text { Determination des ventes }\end{array}$ \\
\hline 3.5 Exaluation continue dumarchs & - & - & $\begin{array}{c}\text { Évaluation continue du marche } \\
-\end{array}$ & - \\
\hline \multicolumn{5}{|l|}{ 4. L'ÉQUIPE ENTREPRENEURIALE } \\
\hline & & & & $\begin{array}{l}\text { Entreprcneurs fondatcurs } \\
\text { Presentation des CV ou commentaires } \\
\text { narratifs concis portant sur: } \\
\text { - Anléculents } \\
\text { - Realisations } \\
\text { - Faillites } \\
\text { Rôle joué par chacun dans lorganigramme }\end{array}$ \\
\hline
\end{tabular}




\begin{tabular}{|c|c|c|c|c|}
\hline $\begin{array}{c}\text { IROOKS \& STEVENS (1987) } \\
\text { sulte }\end{array}$ & DELL'ANIELLO (1987) suile & GASSI: et Al.. (198K) suite & (G(O)D (1989) suite & ONUDI (1978) suite \\
\hline $\begin{array}{l}\text { Déicrmination de la part de marche } \\
\text { Déicrmination des ventes }\end{array}$ & - & $\begin{array}{l}\text { Décrmination de la part de inarche } \\
\text { Décrmination des venies } \\
\text { - } \\
-\end{array}$ & $\begin{array}{l}\text { Décrmination de la part de marche } \\
\text { Délermination des ventes } \\
\text { - }\end{array}$ & $\begin{array}{l}\text { De la concurrence, proluits substituls } \\
\text { Deierminer la base de penétration } \\
\text { Determiner la part de marché } \\
\text { Déerminer les ventes } \\
\text { - } \\
\text { Capacile d'usinc el technologic }\end{array}$ \\
\hline 4. L'EQUIPE ENTREPRENEURIALE & & & & \\
\hline $\begin{array}{l}\text { Presentation des CV } \\
-\quad \text { Anuscedents } \\
-\quad \text { romation } \\
\text { Compriences manquantes dans l'equipe } \\
\text { Responsabilites de chacun }\end{array}$ & $\begin{array}{l}\text { Prescntation des CV } \\
\text { Faite ressortir les capacites et } \\
\text { atouts pour reussis } \\
\text { Presenter les capaciles financierres de } \\
\text { chacin } \\
\text { Deficrminer les responsabilites des } \\
\text { membres de l'Qquipe }\end{array}$ & 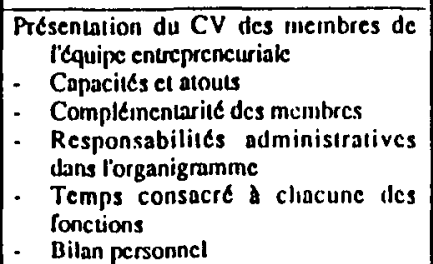 & & \\
\hline
\end{tabular}




\begin{tabular}{|c|c|c|c|c|}
\hline TIMMONS el AL. (1985) sulte & MANCUSO (1985) suite & MCCLAUGIILIN (1985) suile & FORTIN (1986) suile & SIE(BF.L et AL. (1987) suile \\
\hline 5. PLAN MARKETING & & & & \\
\hline $\begin{array}{l}\text { 5.1 Scrilepie globale: } \\
\text { - Comment el quand chaque segment } \\
\text { est developpd } \\
\text { Comment les clients seront } \\
\text { identifies et conisetes } \\
\text { Foree du prodult exploitee (produit. } \\
\text { scrvice, garantic, livraison) }\end{array}$ & & & $\begin{array}{l}\text { SLalćgic marketing } \\
\text { - Comment et quand chaque segment } \\
\text { cst developpe } \\
\text { - Comment les clicnts sont identifits } \\
\text { el altcints } \\
\text { - Force du produit cxploitt (proluit, } \\
\text { scrvicc. garantic, livraison) }\end{array}$ & $\begin{array}{l}\text { Stralégic de yenles dour supdorler la } \\
\text { croissanse } \\
\text { - Licence } \\
\text { - Franchise } \\
\text { - Autocroissance }\end{array}$ \\
\hline $\begin{array}{l}\text { 5.2 Piis: } \\
\text { Le bon prix pour penterer le marche } \\
\text { el faire un profit } \\
\text { Comparaison avec la concurrence } \\
\text { Justifier les écans }\end{array}$ & $\begin{array}{l}\text { Brix: } \\
\text { Politique de cródit } \\
\text { Politique de prix el comparaison } \\
\text { avec les coots }\end{array}$ & $\begin{array}{l}\text { Bris: } \\
\text { Politique de prix el positionnement } \\
\text { du perxluit }\end{array}$ & $\begin{array}{l}\text { Lrix: } \\
\text { Politique de prix } \\
\text { Base de (Ktennination } \\
\text { Prix de revient } \\
\text { Comparaison avec la concurrerce } \\
\text { Marges dle profits } \\
\text { Justification des ecirts }\end{array}$ & $\begin{array}{l}\text { Brix: } \\
\text { Expliquer la suructure de prix } \\
\text { - Politiques d'escomple }\end{array}$ \\
\hline 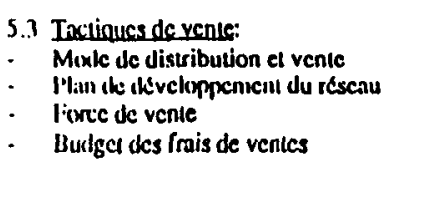 & $\begin{array}{l}\text { Tocliaucs de vente: } \\
\text { Deierminer points de vente } \\
\text { Mente ite distritution } \\
\text { Frais de ventes }\end{array}$ & $\begin{array}{l}\text { Tocliques de venie: } \\
\text { Force de venie }\end{array}$ & $\begin{array}{l}\text { Tacliques de ventes: } \\
\text { - Méthodes de venics } \\
\text { - Circuit de distribution } \\
\text { - Marges conscntics au rescau } \\
\text { - Choix des distributeurs } \\
\text { - Politigues de distribution } \\
\text { - Politiques de crélit }\end{array}$ & $\begin{array}{l}\text { Simicgiede venlest de distributien } \\
\text { - Propres vendeurs } \\
\text { - Agcnts manufacturiers } \\
\text { - Delaillants, distributcurs } \\
\text { - Methale de compensaion } \\
\text { - Qualifications des vendeurs } \\
\text { - Représcntation geographique }\end{array}$ \\
\hline
\end{tabular}




\begin{tabular}{|c|c|c|c|c|}
\hline IIROOKS \& STEVFNS (1987) & DELL'ANIELLO (1987) suile & GASSE et AL.. (198X) suite & (i(1)1) (1989) suite & ONUDI (1978) suile \\
\hline 5. PLAN MARKETING & & & & \\
\hline $\begin{array}{l}\text { Stralckic slobale: } \\
\text { - Comment les clients sont attcints } \\
\text { Forces du produit a exploiter } \\
\text { (conception, performance, emballa- } \\
\text { gc) }\end{array}$ & $\begin{array}{l}\text { Produil: } \\
\text { - Besoin salisfait } \\
\text { - Differenciation } \\
\text { - Forces et faiblesses } \\
\text { - Emballage (conditionnement } \\
\text { - Marque ct crittres de choix }\end{array}$ & $\begin{array}{l}\text { Sunulépic markeging: } \\
\text { - Descriplion de la suragegie globale } \\
\text { - Comment les clicnis sour allcints }\end{array}$ & & \\
\hline $\begin{array}{l}\text { Prix: } \\
\text { Base de determination } \\
\text { Prix de revient } \\
\text { - Pris du marche } \\
\text { Valcur de remplacement de la } \\
\text { fonction } \\
\text { - Comparaison avoc concurrence } \\
\text { Avantage concumenticl? }\end{array}$ & $\begin{array}{l}\text { Prix: } \\
\text { Deiermincr selon la clientele et le type } \\
\text { de produx } \\
\text { (Luxe vs bas de gamme) } \\
\text { - Prix de revient plus } \\
\text { - Prix de pentiration } \\
\text { - Prix d'Ccremage } \\
\text { - Prix de prestige }\end{array}$ & $\begin{array}{l}\text { Prix: } \\
\text { Politique de prix }\end{array}$ & $\begin{array}{l}\text { Prix: } \\
\text { - Discuter de la politique } \\
-\quad \text { Marges de profils } \\
\text { - Comparaison avec les prix de } \\
\text { revient } \\
\text { - Compamison avec la concurrence } \\
\text { - Conditions de venic }\end{array}$ & $\begin{array}{l}\text { Pris: } \\
\text { - Prix monopolistique } \\
\text { - Prix en concurrence } \\
\text { - Possibilite de vendre à perie pendant } \\
\text { une cenaine periode } \\
\text { - Permelle un profit raisonnable, qui } \\
\text { decouragc les cntrants poten liels. }\end{array}$ \\
\hline $\begin{array}{l}\text { Juclisucs de yentecldistribulion } \\
\text { Scrvice postal } \\
\text { - Livtaison par la maison } \\
\text { - Points de vente au détail } \\
\text { - Cours de distribution }\end{array}$ & $\begin{array}{l}\text { Tacliaucs de venteseld distribution } \\
\text { - Force de vente - Comment el } \\
\text { justifier le choix } \\
\text { Description du rescau de } \\
\text { distribution }\end{array}$ & $\begin{array}{l}\text { Tractiques de ventes el distribulion } \\
\text { - Rescaux de distribution } \\
- \text { Points de vente }\end{array}$ & 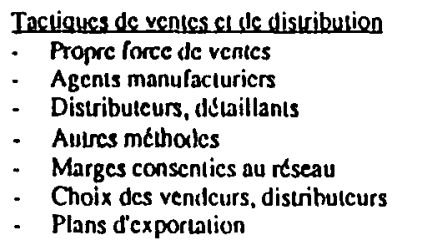 & $\begin{array}{l}\text { Ressau de distribution } \\
\text { - Firme elle-meme, propre force de } \\
\text { vente } \\
\text { - Choix du réscau } \\
\text { - Agcnis manufacturiers } \\
\text { - Disuributcurs ct detaillants } \\
\text { - Coûts }\end{array}$ \\
\hline
\end{tabular}




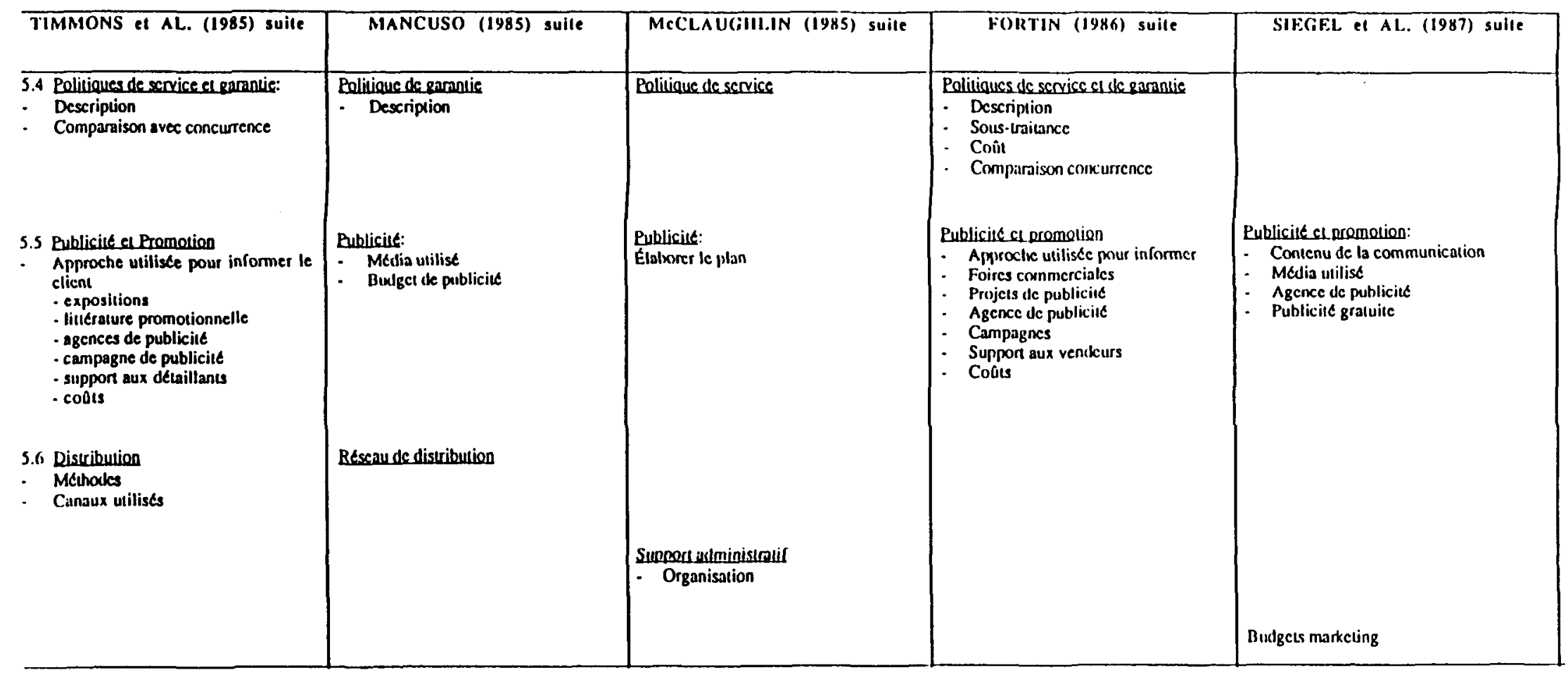




\begin{tabular}{|c|c|c|c|c|}
\hline $\begin{array}{c}\text { BROOKS \& STEVENS (1987) } \\
\text { sulte }\end{array}$ & DELL'ANIELLOO (1987) suile & GASSE: el Al. (1984) suile & (GO)(1) (1989) suite & ONUDI (1978) suite \\
\hline $\begin{array}{l}\text { Service npres renle } \\
\text { - Requis? }\end{array}$ & Bolitioner de srantie es de service & & $\begin{array}{l}\text { Poliliques de scrvice cl de Rarantic } \\
-\quad \text { Description } \\
\cdot \quad \text { Qui le fait } \\
\text { - Comparaison avec concurnence }\end{array}$ & $\begin{array}{l}\text { Scrvice apress venle } \\
-\quad \text { Politiques } \\
-\quad \text { Qui fail quoi? }\end{array}$ \\
\hline $\begin{array}{l}\text { Publicile promotion } \\
\cdot \quad \text { Description }\end{array}$ & 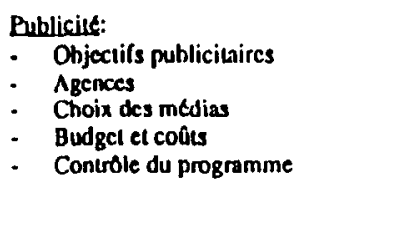 & $\begin{array}{l}\text { Bublicile el promotion } \\
\text { - Objoctifs poursuivis } \\
\text { - Clieniele cille } \\
\text { - Pronnotion au poim de vame } \\
\text { - Publicite } \\
\text { - Choix des medias } \\
\text { - Calcndrier des axtivites } \\
\text { Conesle }\end{array}$ & $\begin{array}{l}\text { Publicile cl promolion } \\
\text { - Programme dinformation } \\
\text { - Agence de publicile } \\
\text { - Madia uitisé } \\
\text { - Busfgel de publicite } \\
\text { - Calcndrier des activites } \\
\text { - Plans pour obicnir de la publicile } \\
\text { gratuics }\end{array}$ & $\begin{array}{l}\text { Mesures de promation } \\
\text { licntifier el déerminer les coats }\end{array}$ \\
\hline & & & & 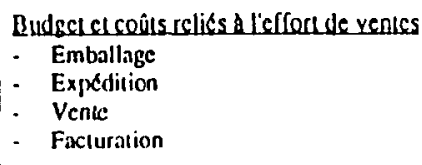 \\
\hline
\end{tabular}




\begin{tabular}{|c|c|c|c|c|}
\hline TIMMONS ef AL. (1985) suile & MANCUSO (1985) suite & MCCLAU(BIILIN (1985) sulte & FORTIN (1986) suile & SIEGEL el AL. (1987) suite \\
\hline $\begin{array}{l}\text { 6. PLAN DE RECHERCHE ET DE } \\
\text { DÉVELOPPEMENT }\end{array}$ & & & & \\
\hline $\begin{array}{l}\text { Idenufier le stalut de développement } \\
\text { el ce qui reste \& fajre } \\
\text { Anticiper les difficulics et les } \\
\text { risques } \\
\text { Plans de developpement d'sutres } \\
\text { produits } \\
\text { Coals de R \& D. } \\
\text { Brevels el maxques de commerce }\end{array}$ & $\begin{array}{l}\text { - Decrire les activités dle R \& D eI } \\
\text { Icur imponance } \\
\text { - Identificr les ressources humaines } \\
\text { - Identifier les nouveaux socteurs de } \\
\text { recherche } \\
\text { - Identificr les subventions } \\
\text { - Identifier tout particnariat }\end{array}$ & & $\begin{array}{l}\text { - Statul de developpenient du } \\
\text { proxluit, ce qui reste à faire } \\
\text { - Difficulies et risclues } \\
\text { - Projels de developpement d'autres } \\
\text { produits } \\
\text { - Financencent des projets }\end{array}$ & $\begin{array}{l}\text { Decrire les plans de développemenl de la } \\
\text { ligne de prouluit } \\
\text { Presenier léquipe de recherche } \\
\text { Brevets, licences, marques de commerce: } \\
\text { effel de la protection }\end{array}$ \\
\hline 7. LE PLAN DES OPERATIONS & & & & \\
\hline $\begin{array}{l}7.1 \text { Molizres premicres: } \\
\text { - Identification des fournisscurs el } \\
\text { sous-Iraiants } \\
\text { - Delais de livraison } \\
\text { - Niveau dinventaire requis } \\
\text { - Coûts des matieres premitres et } \\
\text { lournilures }\end{array}$ & 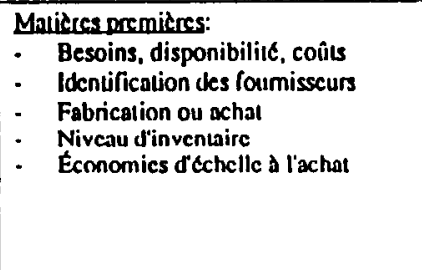 & $\begin{array}{l}\text { Mntictres premiches } \\
\text { Coúts }\end{array}$ & $\begin{array}{l}\text { Manicies premiercs: } \\
\text { Achat ou fabrication }\end{array}$ & $\begin{array}{l}\text { Malicres premitres: } \\
-\quad \text { Source } \\
. \quad \text { Disponibilite } \\
- \text { Volatilite des prix } \\
-\quad \text { Identification des foumisscurs } \\
\text { - Achat ou fabrication }\end{array}$ \\
\hline $\begin{array}{l}7.2 \text { Sile choisj: } \\
\text { Avantages et inconvenicnts } \\
\cdot \quad \text { Coüts } \\
\text { - Disponibilite de main-d'ocurre } \\
\text { - Syndicalisation } \\
\text { - Proximitc des clicnts }\end{array}$ & $\begin{array}{l}\text { Localisation du sile: } \\
\cdot \quad \text { Logique de localisation }\end{array}$ & Disponibilite de main-d'oeluvre & $\begin{array}{l}\text { Silc: } \\
\text { Avantages cl inconvenients } \\
\text { - Aclanandage } \\
\text { - Disponibilité de inain-d'ocuvre } \\
\text { - Proximité fournisscurs ou clicnts }\end{array}$ & $\begin{array}{l}\text { Sitc: } \\
\text { Analyse des forces et faiblesses }\end{array}$ \\
\hline
\end{tabular}




\begin{tabular}{|c|c|c|c|c|}
\hline IIROOKS \& STEVENS (1987) & DELL'ANIFLLLO (1987) suile & GASSE: el Al. (1988) suile & (;0)1) (1989) suile & ONUD1 (1978) suite \\
\hline \multicolumn{5}{|l|}{$\begin{array}{l}\text { 6. PLAN DE RECHERCHE ET } \\
\text { DEVVELOPPEMENT }\end{array}$} \\
\hline & . & & $\begin{array}{l}\text { Identificalion du slatul de } \\
\text { developpement cl ce qui resic a } \\
\text { faire } \\
\text { - Difriculies el risques a prevoir } \\
\text { - Budget de R \& D } \\
\text { - Brevets, marques de commerce. } \\
\text { approbaltions gouvernemeniales } \\
\text { requises }\end{array}$ & \\
\hline \multicolumn{5}{|l|}{ 7. I.E PLAN DES OPERATIONS } \\
\hline $\begin{array}{l}\text { Doil délerminer comment } \\
\text { commercialiser, fabriquer el } \\
\text { distribuer le produit }\end{array}$ & & 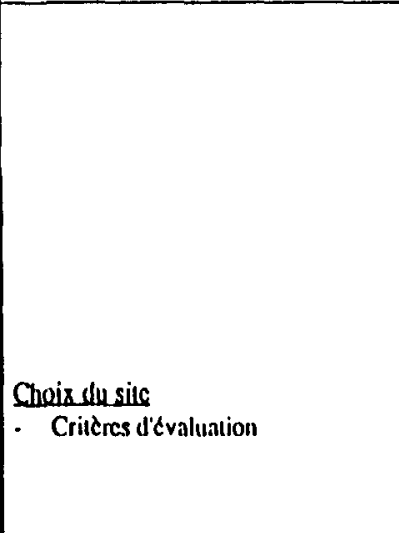 & 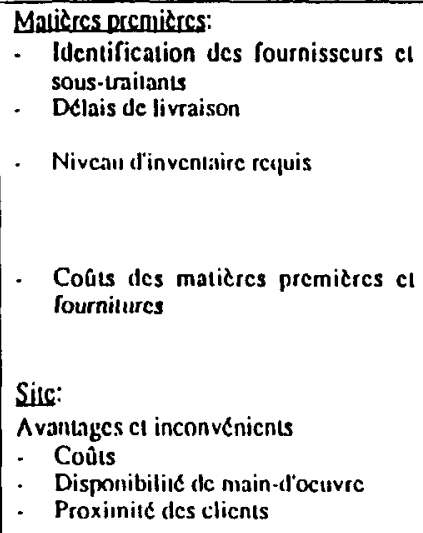 & 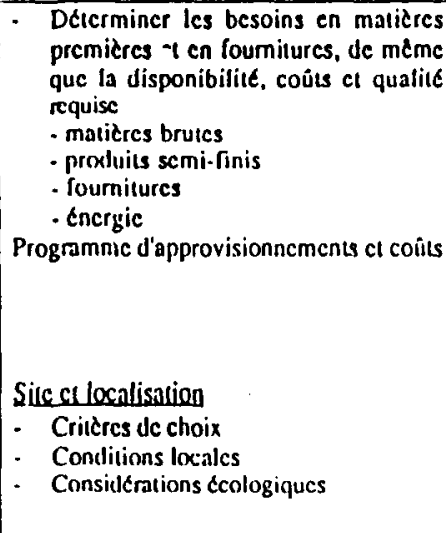 \\
\hline
\end{tabular}




\begin{tabular}{|c|c|c|c|c|}
\hline TIMMONS et AL.(1985) suile & MANCUSO (1985) sulle & MCCLAU(iILIN (1985) suile & FORTIN (1986) suile & SIEGEL el AL. (1987) suile \\
\hline $\begin{array}{l}\text { Accts aux uransports } \\
\text { Disponibilite denergie } \\
\text { Zoinge } \\
\text { Obligalions légales } \\
\text { Circulation routitre } \\
\text { Revenus elc. }\end{array}$ & & & $\begin{array}{l}\text { Accès aux transpons } \\
\text { Situation en relation avec la } \\
\text { concurence }\end{array}$ & \\
\hline $\begin{array}{l}\text { 7.3 Batisses el Gquipements: } \\
-\quad \text { Espace requis - usine et burcaus } \\
\text { Description } \\
\text { - Liste de I'equipement requis } \\
\text { Plan d'aménagement } \\
\text { CoQls }\end{array}$ & $\begin{array}{l}\text { Descriotion Balissci Equincement } \\
-\quad \text { Eqace } \\
\text { - Rythme de fabrication } \\
\text { - Dexcription } \\
\text { - Liste des Equipements } \\
\text { - Capacite } \\
\text { Plan d'amenagement }\end{array}$ & Lisle des cquipements requis & 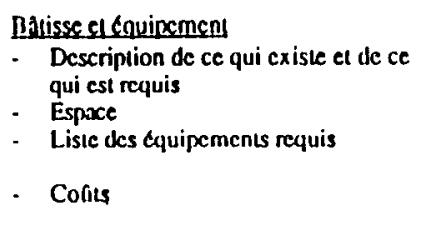 & $\begin{array}{l}\text { Dälisses el squitipement } \\
\text { - Sources } \\
\text { - Nature, Liste } \\
\text { Role } \\
\text { - Capacile d'usine el objectifs de } \\
\text { croissance } \\
\text { - Coúts }\end{array}$ \\
\hline $\begin{array}{l}\text { 7.4 Descriplion des orocessus de } \\
\text { fabcicalionetepols } \\
\text { Detail des codts y inclus frais de } \\
\text { fabrication } \\
\text { Procédures de controle de qualite, } \\
\text { planificalion el controle de } \\
\text { production } \\
\text { Considdrations ecologiques }\end{array}$ & $\begin{array}{l}\text { Descriplion des processus de } \\
\text { fabrication } \\
\text { - Contrōle de qualite }\end{array}$ & Detail des frais de fabrication & $\begin{array}{l}\text { Descriotion des processus de } \\
\text { fabrication } \\
\text { Détail des cools } \\
\text { - Procetdures des controles de la } \\
\text { qualite. approvisionncment. } \\
\text { contrólc }\end{array}$ & \\
\hline $\begin{array}{l}\text { 7.5 Mnin-d'ocume } \\
. \quad \text { Besoins } \\
. \quad \text { Qunlite } \\
\text { Taux el avantages } \\
. \quad \text { Coóts }\end{array}$ & $\begin{array}{l}\text { Main-decurre } \\
-\quad \text { Besoins (syndicat ou non) } \\
-\quad \text { Qualite } \\
-\quad \text { Taux el avantages }\end{array}$ & $\begin{array}{l}\text { Main-d'ocuurce } \\
- \text { Besoins } \\
- \text { Descriptions de taclies } \\
\text { - Taux el avantages } \\
\text { - Coots }\end{array}$ & $\begin{array}{l}\text { Main-d'ocuvre } \\
\text { - Besoins - disponibilité } \\
\text { - Qualité, description de táches } \\
\text { - Taux ct avantagcs } \\
\text { - Formation }\end{array}$ & $\begin{array}{l}\text { Main-d'ocurre } \\
\text { Decrire les besoins }\end{array}$ \\
\hline
\end{tabular}




\begin{tabular}{|c|c|c|c|c|}
\hline $\begin{array}{l}\text { BROOKS \& STEVENS (1987) } \\
\text { sulle }\end{array}$ & DFLL'ANIELLIO (1987) sulle & (iASSE el AL. (1984) suite & (60)0 (1989) suite & ONUDI (1978) suite \\
\hline . & $\begin{array}{l}\text { Descriotion du processus } \\
\text { - Faire un croquis de l'aménagement }\end{array}$ & 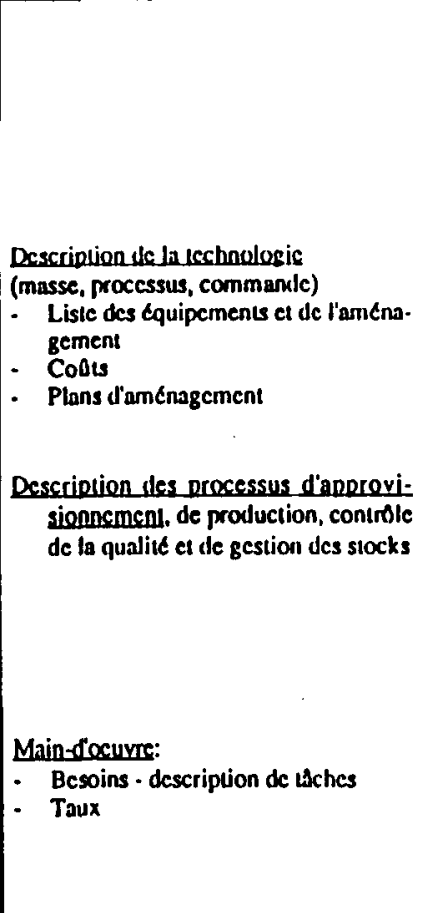 & 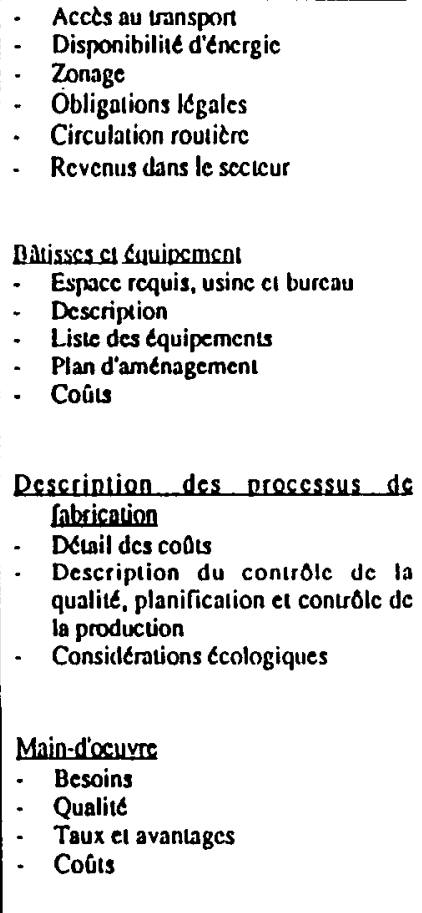 & 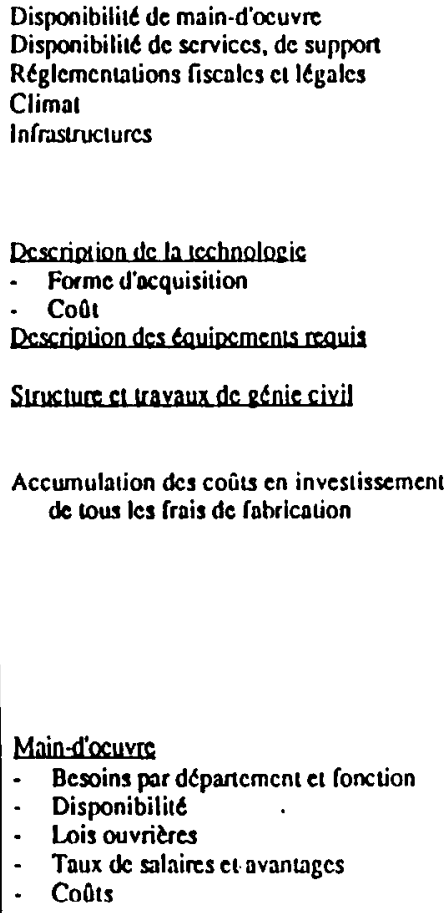 \\
\hline
\end{tabular}




\begin{tabular}{|c|c|c|c|c|}
\hline TIMMONS el AL. (1985) sulie & MANCUSO (1985) suite & McClAU(ill.IN (1985) suile & FORTIN (1986) suile & SIE(iEL et AL. (1987) suite \\
\hline \multicolumn{5}{|l|}{$\begin{array}{l}\text { 8. LE PLAN DES RESSOURCES } \\
\text { HUMAINES }\end{array}$} \\
\hline $\begin{array}{l}8.1 \text { lisnificr les posics-cies dans lorga- } \\
\text { nisalion } \\
\text { Organigramme }\end{array}$ & Organigramme & $\begin{array}{l}\text { Bosicscles: } \\
\text { Organigramme }\end{array}$ & $\begin{array}{l}\text { Pusles de dircscion } \\
\text { Organigramınc }\end{array}$ & $\begin{array}{l}\text { Bosles de discelion } \\
\text { Organignamme }\end{array}$ \\
\hline $\begin{array}{ll}\text { 8.2 } & \text { Pcrsonnel-cle } \\
- & \text { Decrire responsabilites } \\
- & \text { Realisations } \\
- & \text { Inclure CV } \\
- & \text { Complementarile }\end{array}$ & Inclure CV & $\begin{array}{ll}\text { Personncl-cle } \\
- & \text { Decrire responsabilite } \\
- & \text { Realisations } \\
- & \text { Inclure CV }\end{array}$ & $\begin{array}{l}\text { Personnclude dircstion } \\
\text { - Responsibilites } \\
\text { - Complementarite }\end{array}$ & Pcrsonncl-cle \\
\hline $\begin{array}{l}\text { 8.3 Plan de remuntration el du parage } \\
\text { de la oromriele } \\
\text { - Salaircs } \\
\text { - Participation }\end{array}$ & $\begin{array}{l}\text { - Salaires el tous les avantages, plan } \\
\text { de participation, bonis }\end{array}$ & & $\begin{array}{l}\text { Salaircs } \\
\text { - Participation au capital } \\
\text { - Investisscment }\end{array}$ & . \\
\hline $\begin{array}{l}\text { 8.4 Aulres invessisseurs el part de } \\
\text { proboides }\end{array}$ & & & & $\begin{array}{l}\text { Informaticn sur les investisscurs aclits } \\
\text { - Capital et expertise }\end{array}$ \\
\hline $\begin{array}{l}\text { 8.5 Composition du conscill d'adminis: } \\
\text { Imlion }\end{array}$ & Identifier les dlirecteurs & & $\begin{array}{l}\text { Composition du conscil cl comps- } \\
\text { Iences }\end{array}$ & Dirocicurs \\
\hline \multicolumn{5}{|l|}{ 8.6 Droils des aclionnaires el restriclion } \\
\hline $\begin{array}{l}8.7 \text { Les_organismes de conseil el de } \\
\text { subdon }\end{array}$ & Consultants, inclure les contrats & & Services professionnels d'appoints & Conseiliers \\
\hline
\end{tabular}




\begin{tabular}{|c|c|c|c|c|}
\hline HROOKS \& STEVENS (1987) & DELL'ANIELLO (1987) suife & (iASSE: et AL. (1DBK) smite & (6001) (1989) sulte & ONUDI (1978) suite \\
\hline $\begin{array}{l}\text { 8. LE PLAN DES RESSOURCES } \\
\text { HUMAINES }\end{array}$ & & & & \\
\hline Discult dans l'equipe enoepreneuriale & Disculd dans I'equipe enurcprencuriale & Discult dins l'cquipe enureprencuriale & 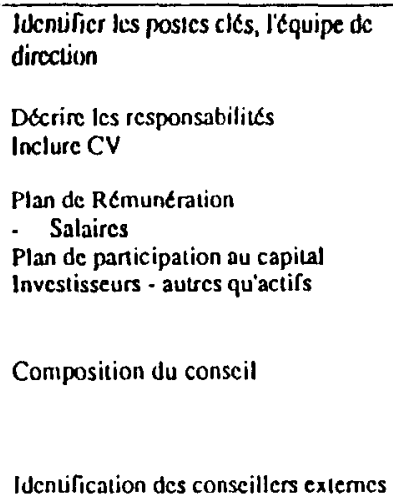 & Inclus au chapiure precctdant \\
\hline
\end{tabular}




\begin{tabular}{|c|c|c|c|c|}
\hline TIMMONS el AL. (1985) sulie & MANCUSO (1985) suile & MCCL.AUGIILIN (1985) suite & FORTIN (1986) suile & SIEGEL el AL. (1987) suile \\
\hline $\begin{array}{l}\text { 9. LE CALENDRIER DE REALISA- } \\
\text { TIONS ET LE PLAN DE } \\
\text { GESTION DES RISQUES }\end{array}$ & & & & \\
\hline 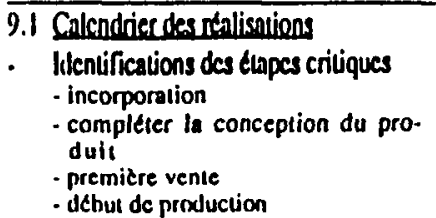 & Aucune mention & Aucune mention & 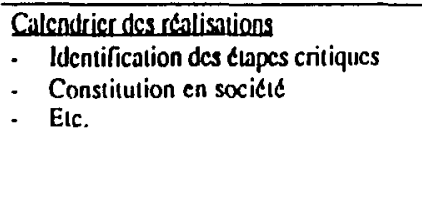 & 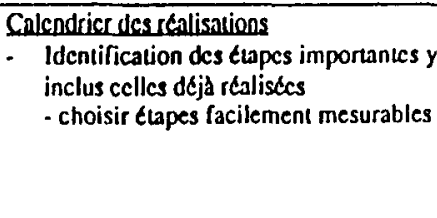 \\
\hline Éublir les behcanciers pour chacun & & & Éablir Ics echeancicrs pour chacun & $\begin{array}{l}\text { - Établir les \&chcanciers } \\
\text { - prevoir ICs delais provenant de } \\
\text { l'externe }\end{array}$ \\
\hline $\begin{array}{l}\text { Disculer des elupess susceppibles de } \\
\text { crérer problemes el des impacts }\end{array}$ & & & $\begin{array}{l}\text { - Optrations pouvant causcr un } \\
\text { deccallage cl correcuifs }\end{array}$ & - Ambiticux mais réalisables \\
\hline 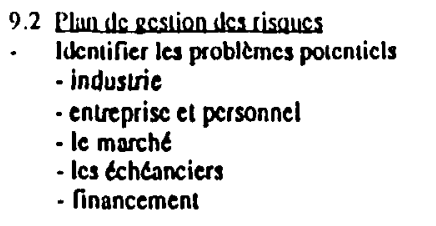 & Aucune mention & Aucunc inention & $\begin{array}{l}\text { Plande ecslion des risques } \\
\text { Identification des risques el } \\
\text { problenics } \\
\text { - sccleur industricl } \\
\text { - marche } \\
\text { - financement }\end{array}$ & Aucune mention \\
\hline Descrirc les plans de conlingence & & & - Plans de contingence & \\
\hline
\end{tabular}




\begin{tabular}{|c|c|c|c|c|}
\hline $\begin{array}{c}\text { DIROOKS \& STEVENS (1987) } \\
\text { suile }\end{array}$ & DFI.L.'ANIFLLLO (1987) suile & GASSE: el Al.. (198K) suite & (iOO)1) (19K.) suile & ONUDI (1978) suite \\
\hline \multicolumn{5}{|l|}{$\begin{array}{l}\text { 9. LE CALENDRIER DE REALISA. } \\
\text { TION ET LE PLAN DE GESTION } \\
\text { DES RISQUES }\end{array}$} \\
\hline $\begin{array}{l}\text { 9.1 Calendiecedesstaliemlions } \\
\text { - Identification des Etapes critiques et } \\
\text { objoctifs } \\
\text { - marketing } \\
\text { - operations } \\
\text { - rinances }\end{array}$ & Aucune mention & Aucune mention & $\begin{array}{l}\text { Calcadricr de realisasion } \\
\text { Identification des étapes critiques }\end{array}$ & $\begin{array}{l}\text { Colcadricer dectálisation } \\
\text { Dérinir les difrerentes etapes a realiser }\end{array}$ \\
\hline & & & & $\begin{array}{l}\text { - Éablir le temps requis pour les } \\
\text { tealiser }\end{array}$ \\
\hline & & & $\begin{array}{l}\text { - Disculcr ce qui a éte fais pour les } \\
\text { renconurer }\end{array}$ & \\
\hline & & & - Disculer des plans de contingence & Réajuster en cours de réalisation \\
\hline 9.2 Plan de gestion des risaues & & & $\begin{array}{l}\text { Elande session des risques: } \\
\text { - Identification des problemes el } \\
\text { risques }\end{array}$ & \\
\hline Aucune mention & Aucune mention & Aucune mention & 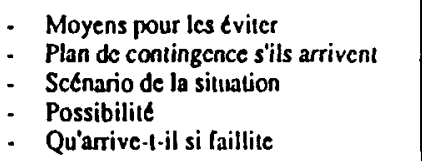 & Aucune mention \\
\hline
\end{tabular}




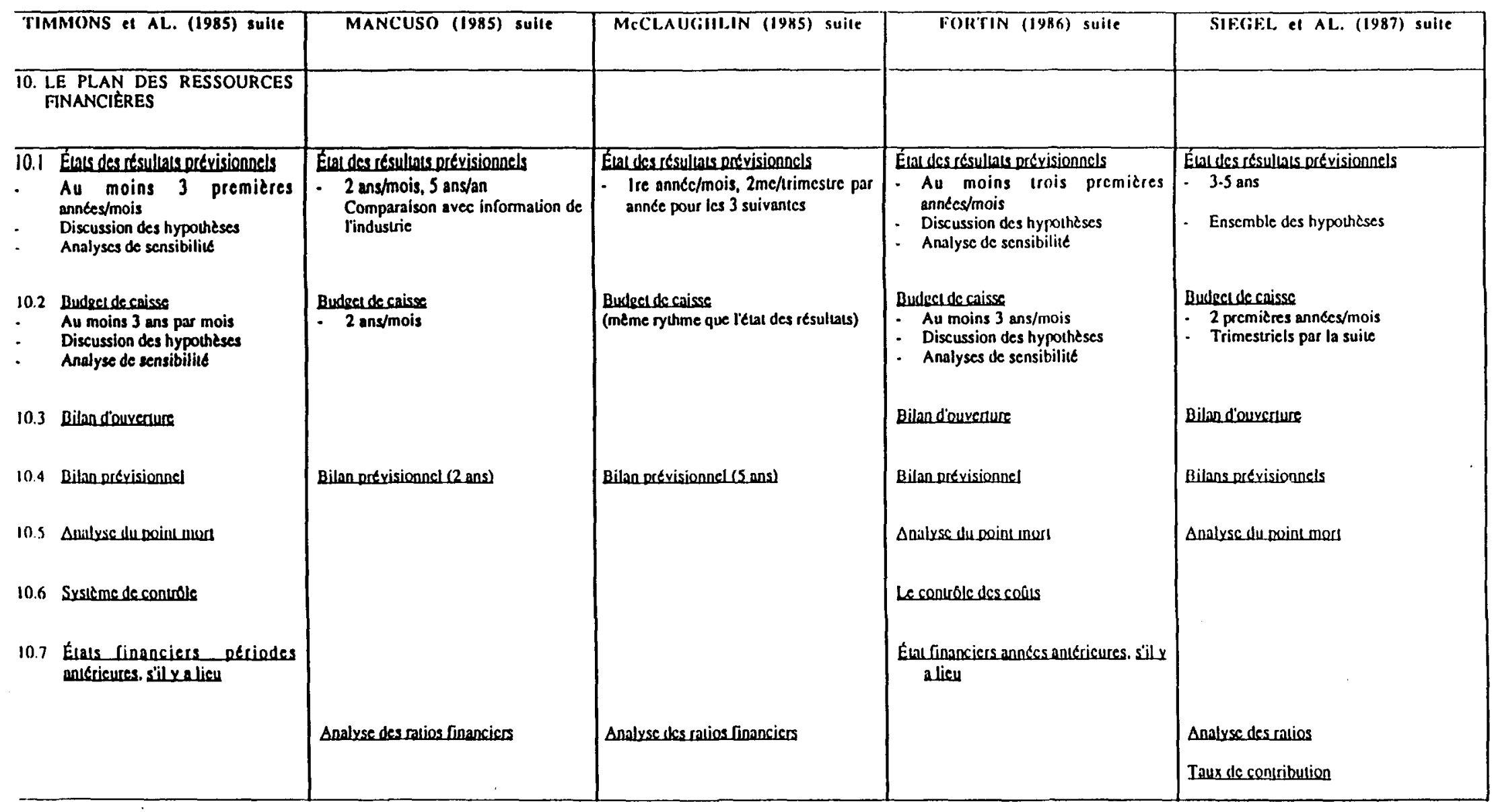




\begin{tabular}{|c|c|c|c|c|}
\hline IIROOKS \& STEVENS (1987) & DFLL'ANIELLO (1987) suite & (iASSE: et AL. (1988) suite & (60OD) (1989) suile & ONUDI (1978) suite \\
\hline $\begin{array}{l}\text { 10. LE PLAN DES RESSOURCES } \\
\text { FNANCIERES }\end{array}$ & & & & \\
\hline 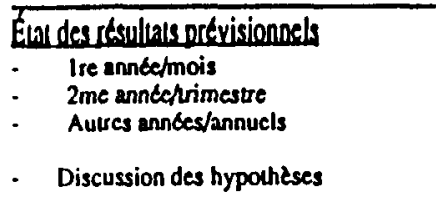 & 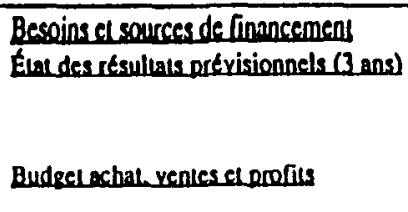 & 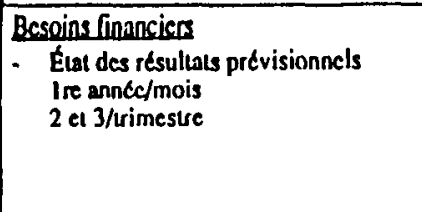 & 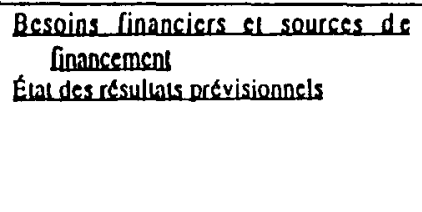 & 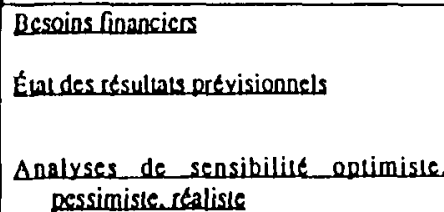 \\
\hline $\begin{array}{l}\text { Rudgec decaisse } \\
\text { Meme rythme que l'état des resullats }\end{array}$ & $\begin{array}{l}\text { Budrec de caisse par mois (1) and } \\
\text { Bilan douvenure }\end{array}$ & Previsions de urssoreris & Budeci de caisse & Bulgel de caisse \\
\hline Dihau arevisionuclannece & Analuse du poinu mors & 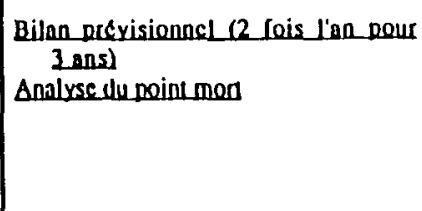 & 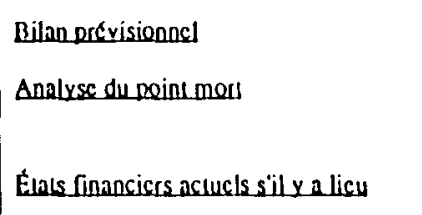 & $\begin{array}{l}\text { Bilan prévisionncl } \\
\text { Analysse du poine men }\end{array}$ \\
\hline Analysedes ravios & Analyse des matios & $\begin{array}{l}\text { Analyse iks ratios } \\
\text { Sources de financemens }\end{array}$ & & $\begin{array}{l}\text { Analyse des ratios } \\
\text { Betour sur investissemens }\end{array}$ \\
\hline
\end{tabular}


ANNEXE 3

SYNTHESE DES RECHERCHES CONSULTEES 
SYNTHESE DES RECHERCHES SUR LA PLANIFCATION STRATEgIQUE DANS LES PME

Présence de la planification stratégique dans les PME

\begin{tabular}{|c|c|c|c|c|c|c|}
\hline Recherches & Taille & $\begin{array}{l}\text { Echantition } \\
\text { Type } \\
\text { dentreprise }\end{array}$ & Particulatiés & Méthodołogie & $\begin{array}{l}\text { Objectifs de } \\
\text { rétude }\end{array}$ & Réschats \\
\hline 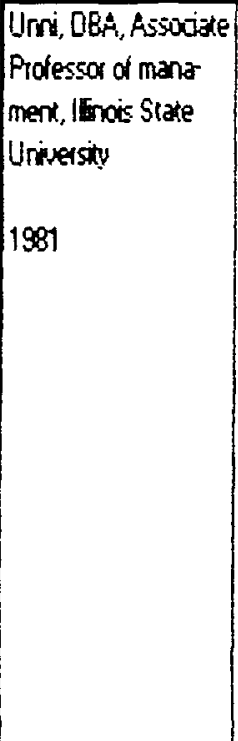 & 120 & $\begin{array}{l}\text { Hanufactures: } \\
62 \text { minoritaines } \\
58 \text { monminoritaies }\end{array}$ & $\begin{array}{l}\text { Existant depuis au } \\
\text { morrs } 2 \text { ans }\end{array}$ & $\begin{array}{l}\text { Questionare de upe } \\
\text { Likert }\end{array}$ & 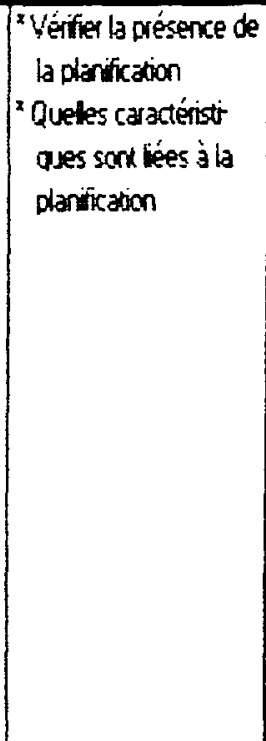 & 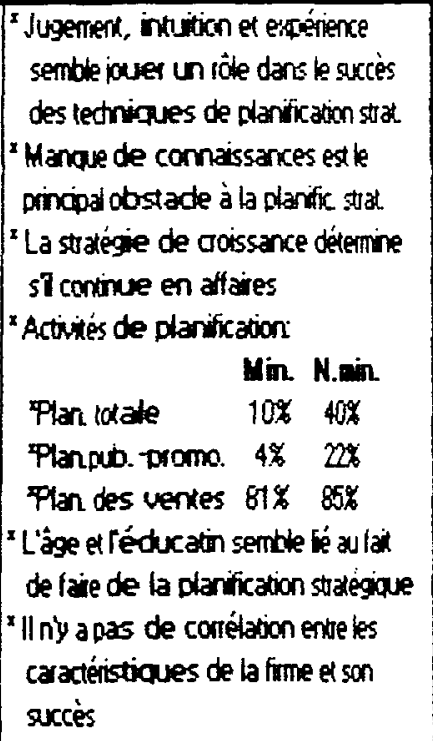 \\
\hline $\begin{array}{l}\text { Seton a Van Auken } \\
1982\end{array}$ & 357 & $\begin{array}{l}\text { Secters difterents } \\
\text { au Texas }\end{array}$ & & $\begin{array}{l}\text { Questionates da } \\
\text { entewues } \\
{ }^{4} 4 \text { questions }\end{array}$ & $\begin{array}{l}\text { Classifies ces enter } \\
\text { prises seton le niveau } \\
\text { de planification }\end{array}$ & 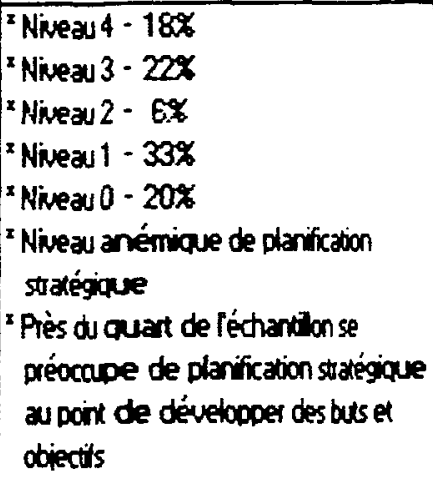 \\
\hline $\begin{array}{l}\text { Stonet, Charkes } R \text {. } \\
1983\end{array}$ & 62 & Hanufactures & 2 à 150 employes & $\begin{array}{l}\text { Entovere: questionaire } \\
\text { en } 4 \text { paties }\end{array}$ & $\begin{array}{l}\text { Estce que la plarifica } \\
\text { ton statégioues est } \\
\text { ưtisée dans les P.E.? }\end{array}$ & 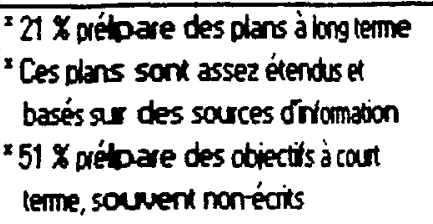 \\
\hline
\end{tabular}


SYNTHESE OES RECHERCHES SUR LA PLANIFICATION STRATEgIQUE DANS LES PME (sUite)

Caractéristiques de la planification stratégique dans les PME

\begin{tabular}{|c|c|c|c|c|c|c|}
\hline Recherches & Talle & $\begin{array}{l}\text { Echantilion } \\
\text { Type } \\
\text { dentseprise }\end{array}$ & Particularités & Méthodologie & $\begin{array}{l}\text { Objectifs de } \\
\text { rétude }\end{array}$ & Réscllats \\
\hline $\begin{array}{l}\text { Jiness } \\
1982\end{array}$ & & $\begin{array}{l}\text { Ent. de services (22) } \\
\text { Manufactures (147 }\end{array}$ & $\begin{array}{l}\text { Avant enoe } 2 \text { et } \\
141 \text { arrees }\end{array}$ & $\begin{array}{l}\text { Questionares pal la } \\
\text { poste }\end{array}$ & 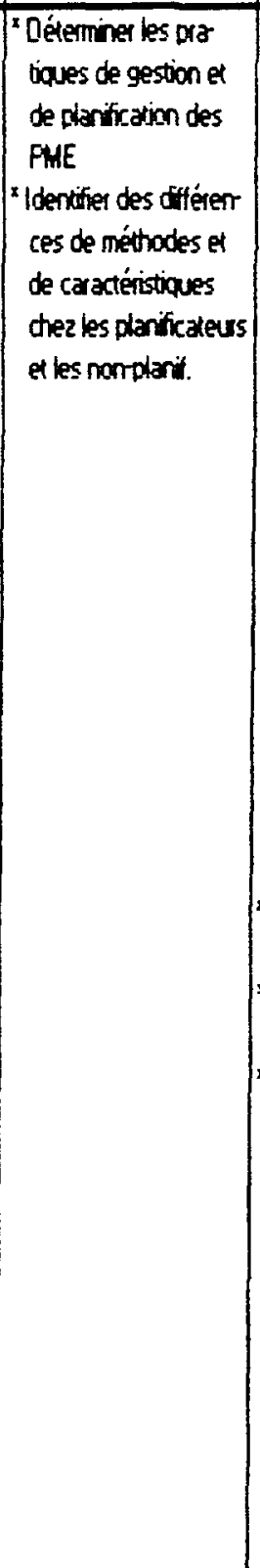 & 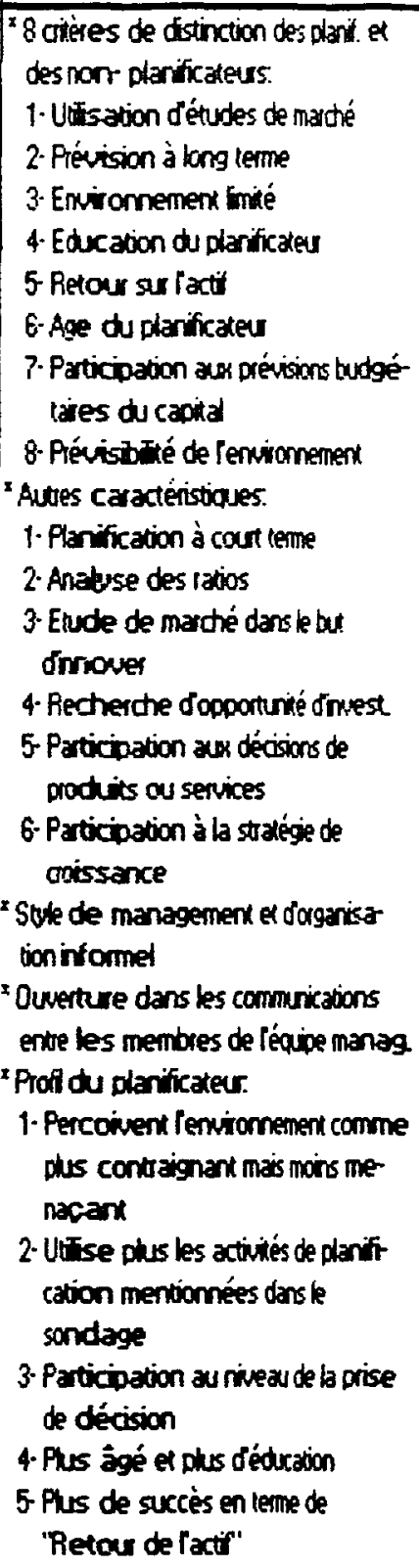 \\
\hline
\end{tabular}


SYNIHESE DES RECHERCHES SUR LA PLANIFICATION STRATEGIQUE DANS LES PME (suite)

Impact de la planification stratégique dans les PME

\begin{tabular}{|c|c|c|c|c|c|c|}
\hline Recherches & Taille & $\begin{array}{l}\text { Echantilion } \\
\text { Trpe } \\
\text { dentreprise }\end{array}$ & Particularités & Méthadalogie & $\begin{array}{l}\text { Obiectifs de } \\
\text { rétude }\end{array}$ & Résullats \\
\hline $\begin{array}{l}\text { Robinan, Wham el } \\
\text { Litleiotn } \\
1951\end{array}$ & & $7 \mid \begin{array}{l}\text { Entreprises ayark } \\
\text { consulé le SBCD }\end{array}$ & & 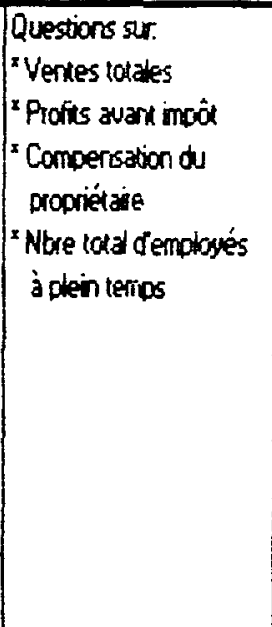 & 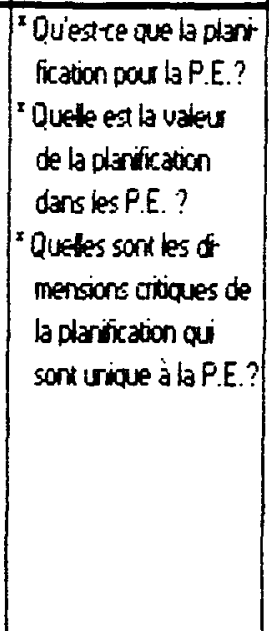 & 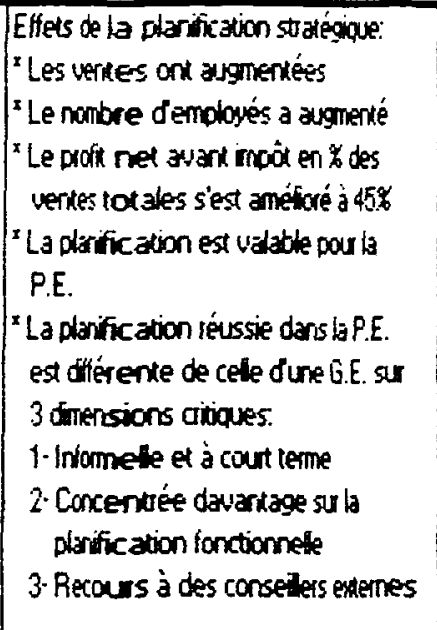 \\
\hline $\begin{array}{l}\text { Rotinson, Peace, } \\
\text { Vozizis et Mescon } \\
1984\end{array}$ & & $\begin{array}{l}\text { Détalank (37) } \\
\text { Entt. services (14) }\end{array}$ & 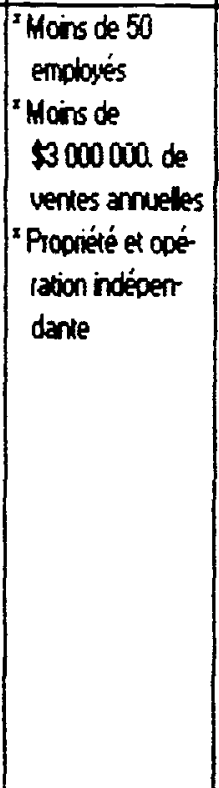 & 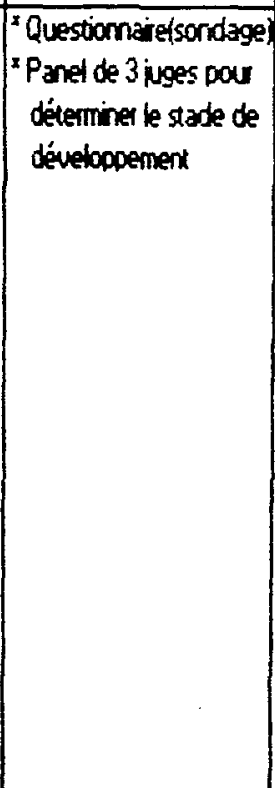 & 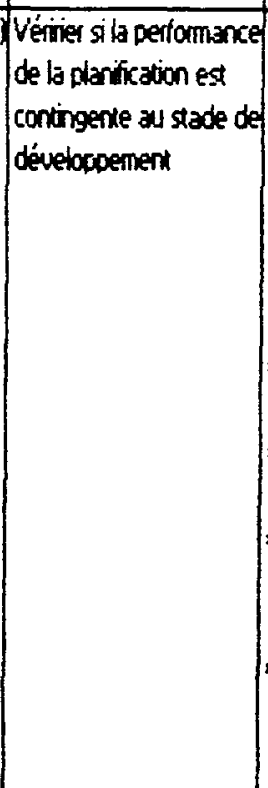 & 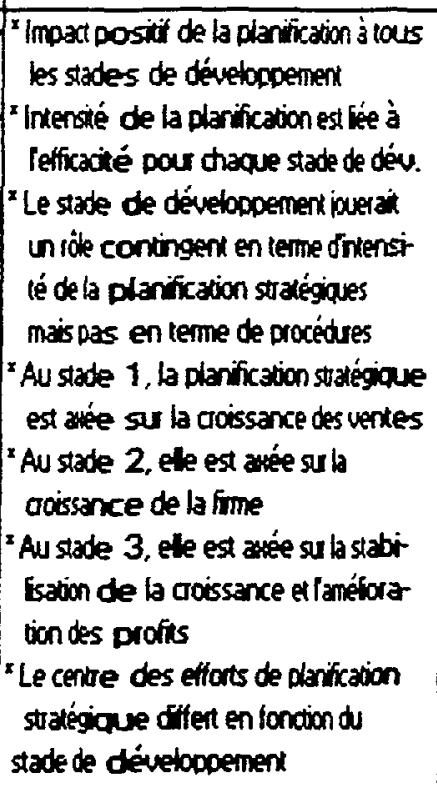 \\
\hline $\begin{array}{l}\text { Robinson, Logan et } \\
\text { Salem } \\
1986\end{array}$ & & $\begin{array}{l}\text { Détalants indéper } \\
\text { dants de fa a menta } \\
\text { tion }\end{array}$ & $\begin{array}{l}\text { Défrition de la } \\
\text { S.BA. }\end{array}$ & $\begin{array}{l}{ }^{2} \text { Questiomiries par to } \\
\text { poste } \\
\times 3 \text { vpes de questions }\end{array}$ & 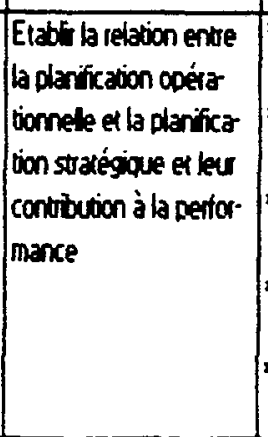 & 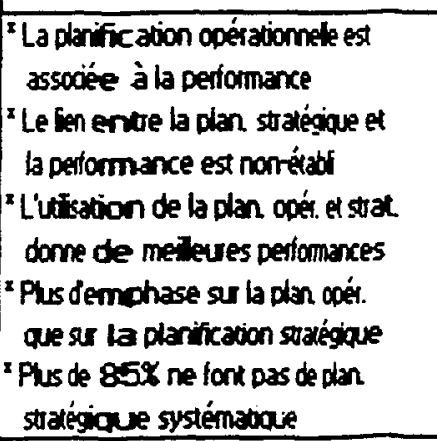 \\
\hline
\end{tabular}




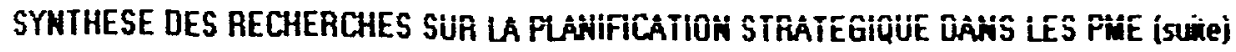

Impact de la planification stratégique dans les PME (suite)

\begin{tabular}{|c|c|c|c|c|c|c|}
\hline Recherches & Talle & $\begin{array}{l}\text { Echantillon } \\
\text { Type } \\
\text { ofentreprise }\end{array}$ & Particularités & Méthodologie & $\begin{array}{l}\text { Objectifs de } \\
\text { rétude }\end{array}$ & Résultats \\
\hline $\begin{array}{l}\text { Shader, Hulford et } \\
\text { Bladtum } \\
1989\end{array}$ & & $\begin{array}{l}\text { Hanufactures (31) } \\
\text { Detadlants (35) } \\
\text { Ento. de services (311) }\end{array}$ & & 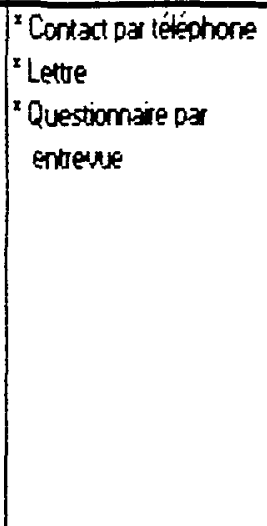 & 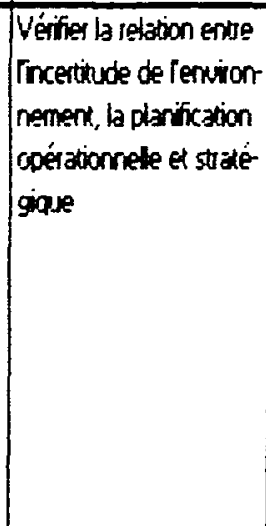 & 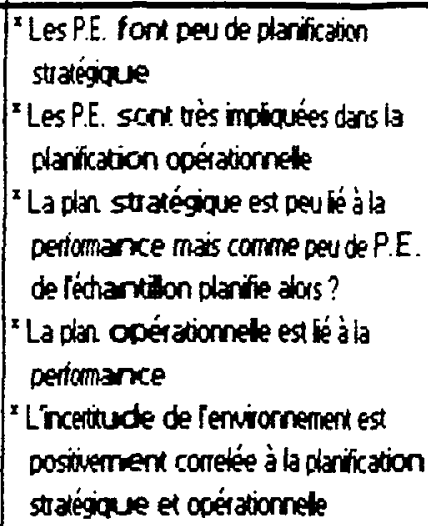 \\
\hline
\end{tabular}


SYNTHESE DES RECHERCHES SUR LA PLANIFICATION STRATEGIQUE DANS LES PME (suite)

Contenu de la planification stratégique dans les PME

\begin{tabular}{|c|c|c|c|c|c|c|}
\hline Recherches & Talle & $\begin{array}{l}\text { Echantiton } \\
\text { Type } \\
\text { Sentreprise }\end{array}$ & Particudarités & Méthodologie & $\begin{array}{l}\text { Obiectifs de } \\
\text { rétude }\end{array}$ & Résuttats \\
\hline $\begin{array}{l}\text { Perty } \\
1960\end{array}$ & & 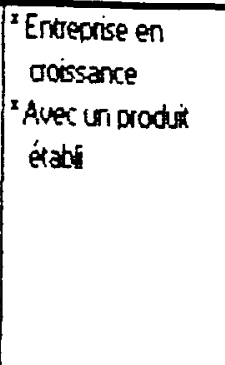 & $\begin{array}{l}1 \text { ou } 2 \text { propriétares } \\
\text { crement toutes les } \\
\text { décisions }\end{array}$ & $\begin{array}{l}\text { Etude de cas } \\
\text { (exaloratione) }\end{array}$ & 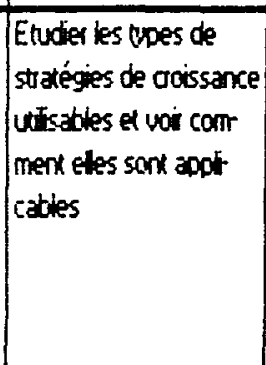 & 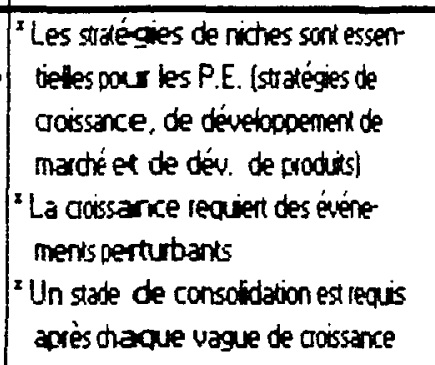 \\
\hline $\begin{array}{l}\text { Calori } \\
1955\end{array}$ & & $\begin{array}{l}\text { Indivtieles. } \\
\text { "Chauffage sclare } \\
\text { "Electriaté solare }\end{array}$ & & Questiomaile & 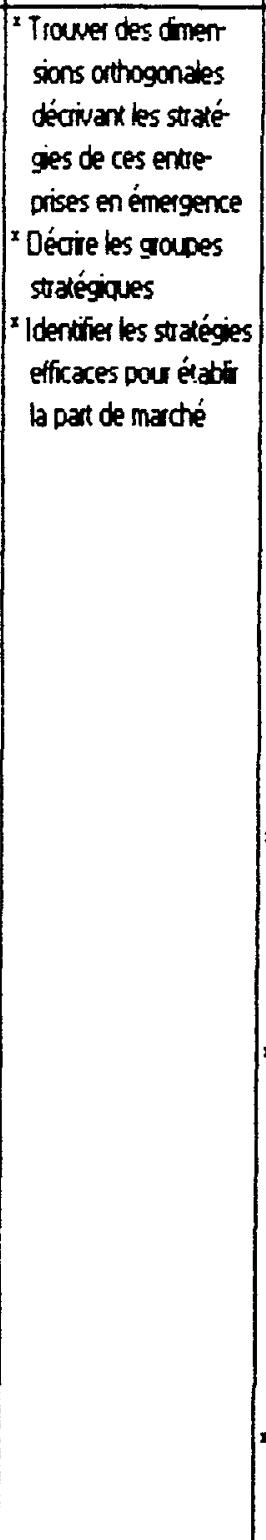 & 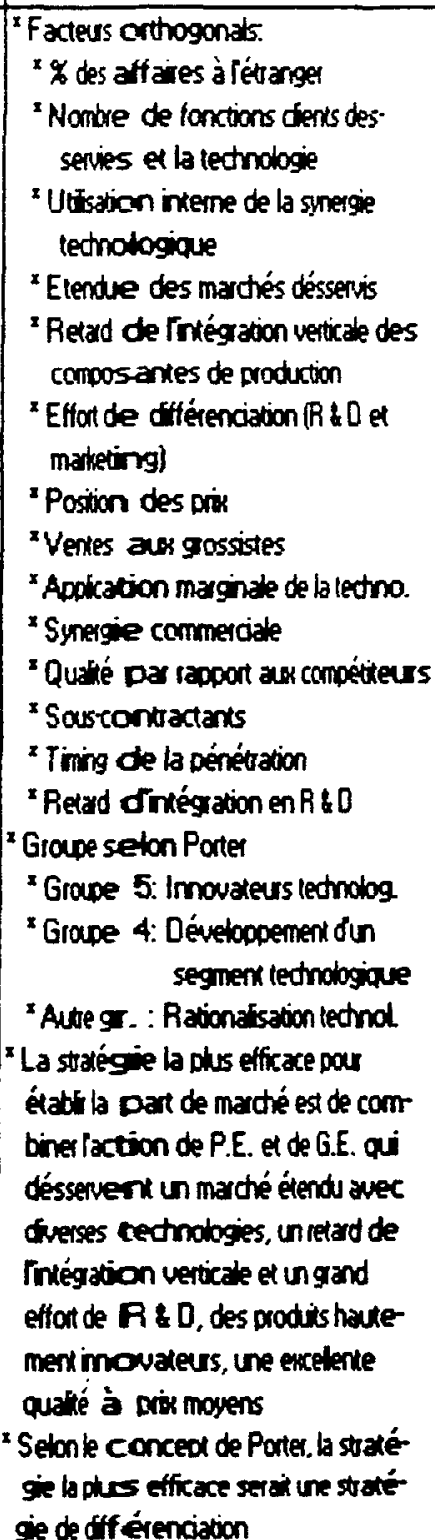 \\
\hline
\end{tabular}




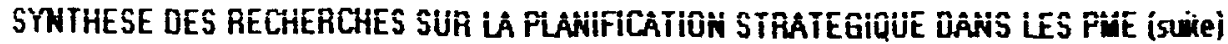

Contenu de la planification stratégique dans les PME (suite)

\begin{tabular}{|c|c|c|c|c|c|c|}
\hline Recherches & Talle & $\begin{array}{l}\text { Echantillon } \\
\text { Type } \\
\text { dentreprise }\end{array}$ & Particularités & Méthodologie & $\begin{array}{l}\text { Obijectifs de } \\
\text { rétude }\end{array}$ & Résultats \\
\hline Darig & & $\begin{array}{l}\text { Indetije: } \\
\text { 'Vêtement } \\
\text { x Fonderie et fatrica } \\
\text { tion de métaws }\end{array}$ & $\begin{array}{l}\text { De } 15 \text { à } 280 \\
\text { encloyes }\end{array}$ & $\begin{array}{l}\text { Questionares pal b } \\
\text { poste }\end{array}$ & 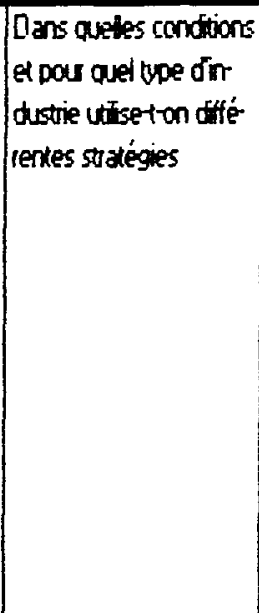 & 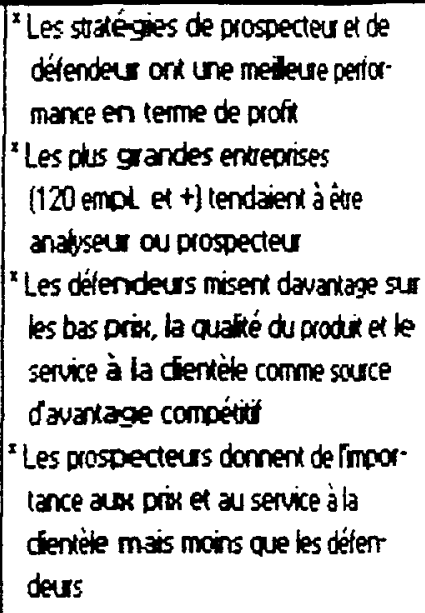 \\
\hline
\end{tabular}


SYNTHESE DES RECHERCHES SUR LA PLANIFICATION STRATEGIQUE DANS LES PME (sLite)

Processus de planification stratégique dans les PME

\begin{tabular}{|c|c|c|c|c|c|c|}
\hline Recherches & Talle & $\begin{array}{l}\text { Echantilion } \\
\text { Troe } \\
\text { dentreprise }\end{array}$ & Particulatiés & Méthodologie & $\begin{array}{l}\text { Obiectifis de } \\
\text { rétude }\end{array}$ & Résollats \\
\hline $\begin{array}{l}\text { Sdiman et Seeger } \\
1966\end{array}$ & 220 & (Classée pa inc) & 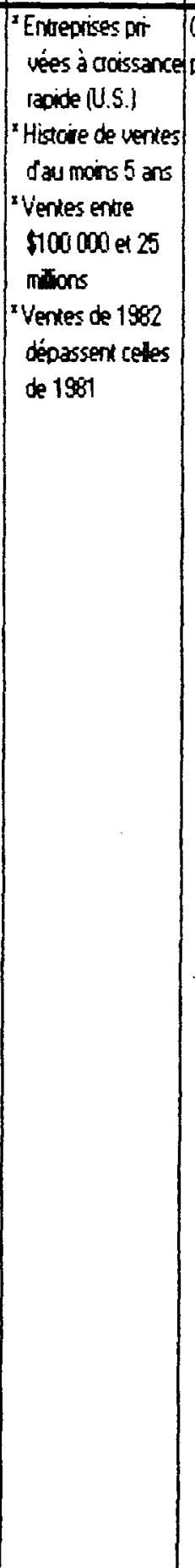 & $\begin{array}{l}\text { Questionate pa la } \\
\text { poste }\end{array}$ & 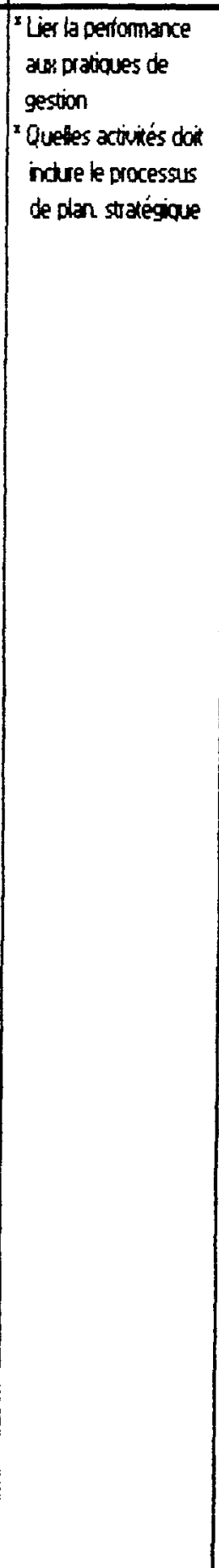 & 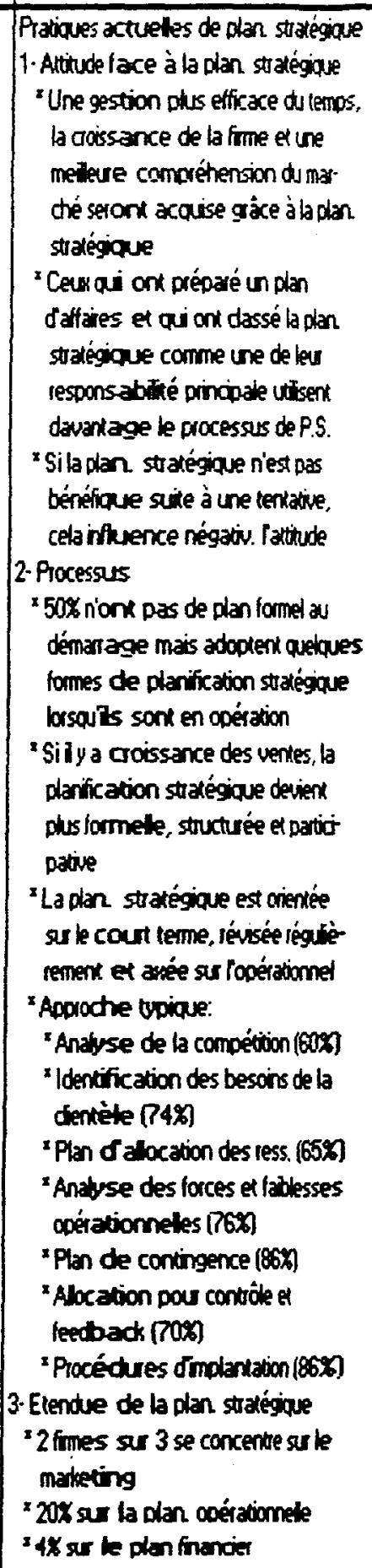 \\
\hline
\end{tabular}


SYNTHESE DES RECHERCHES SUUR LA PLAHIFCAIION STRATEGIQUUE GÂNS LES PHA isuiei

Processus de planification stratégique dans les PME (suite)

\begin{tabular}{|c|c|c|c|c|c|c|}
\hline Recherches & Taile & $\begin{array}{l}\text { Edrantilion } \\
\text { Type } \\
\text { dentreprise }\end{array}$ & Particularités & Méthodologie & $\begin{array}{l}\text { Objectifs de } \\
\text { rétude }\end{array}$ & Résultats \\
\hline $\begin{array}{l}\text { Schuman et Seger } \\
1566 \\
\text { isitej }\end{array}$ & & & & & & 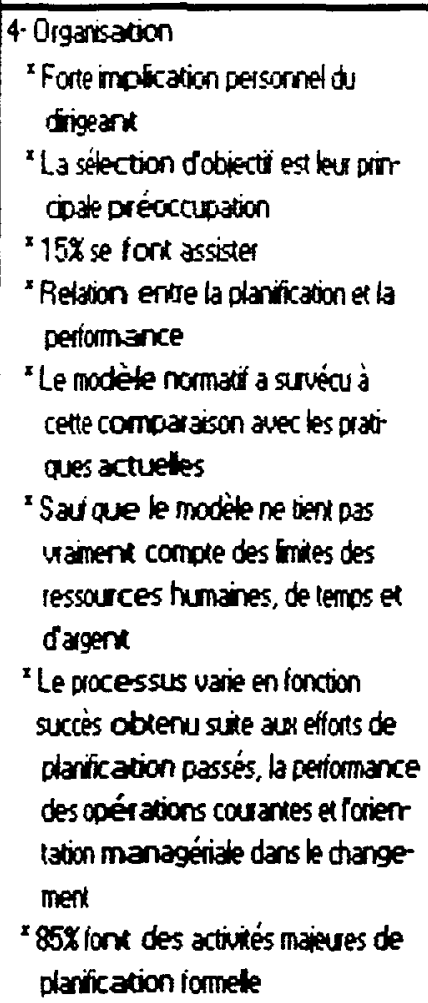 \\
\hline
\end{tabular}

